\title{
Updated checklist of the Umbelliferae of Middle Asia and Kazakhstan: nomenclature, synonymy, typification, distribution
}

\author{
M. G. Pimenov \\ Botanical Garden, Lomonosov Moscow State university, Vorobjevy Gory, Moscow, 117234, Russian Federation. \\ E-mails:mgpimenov@mail.ru,mgpimenov@gmail.com
}

Keywords: Apiaceae, carrot family, endemic species, Kazakhstan, lectotypification, Middle Asia, phytogeography, synonyms, systematics, taxonomy.

Summary. The region of Middle Asia and Kazakhstan with 107 genera, 459 species and 204 endemic species is one of essential Umbelliferae diversity centers not only in Asia, but also in the world. The biggest Umbelliferae genera in the region are Ferula (100 species), Seseli (49), Elwendia (19), Bupleurum (18), Elaeosticta (18), Prangos (16), Semenovia (16). The diversity of the family by country is as follows: Kazakhstan ( 82 genera -211 species -28 endemic species), Uzbekistan (68 - $200-18$, respectively), Kyrgyzstan (65 - $192-29)$, Tajikistan (65 - 176 - 20), and Turkmenistan $(51-122-9)$. The latter differs considerably from other countries of the region not only in lesser diversity, but also in generic and specific sets, approaching the features of Iranian Umbelliferae. The distribution of species was described with regard to provinces of all five countries. The list of endemic species for each country was compiled on the basis of field, herbarium and published data. There are 16 endemic genera in Middle Asia and Kazakhstan, including Astomatopsis, Autumnalia, Fergania, Kafirnigania, Karatavia, Komarovia, Kuramosciadium, Lipskya, Mogoltavia, Paulita, Pilopleura, Schtschurowskia, Sclerotiaria, Sphaenolobium, Sphaerosciadium, and Tschulaktavia, 6 other genera being subendemics. Across the region the important border between Middle Asian (eastern part of SW Asian floristic province of the Mediterranean type) and Central Asian phytochoria passes; the former being considerably richer in the Umbelliferae than the latter.

\section{Обновленный конспект зонтичных (Umbelliferae) Средней Азии и Казахстана: номенклатура, синонимия, типификация, распространение}

\author{
М. Г. Пименов \\ Ботанический сад Московского государственного университета им. М. В. Ломоносова, \\ Воробьевы Горы, г. Москва, 117234, Россия
}

Ключевые слова: зонтичные, Казахстан, лектотипификация., синонимы, систематика, Средняя Азия, таксономия, фитогеография, эндемичные виды, Apiaceae.

Аннотация. Регион Средней Азии и Казахстана со 107 родами, 459 видами и 204 эндемичными видами представляет собой один из важнейших центров разнообразия Umbelliferae не только в Азии, но и в мировом масштабе. Крупнейшими родами зонтичных в регионе являются Ferula (100 видов), Seseli (49), Elwendia (19), Bupleurum (18), Elaeosticta (18), Prangos (16), Semenovia (16). В зависимости от страны, разнообразие семейства распределяется следующим образом: Казахстан ( 82 рода - 211 видов - 28 эндемичных видов), Узбекистан $(68-200-18$ соответственно), Кыргызстан $(65-192-29)$, Таджикистан $(65-176-20)$ и Туркменистан $(51-$ 122 - 9). Последняя отличается от других стран региона не только меньшим разнообразием, но и существенно другим родовым и видовым набором, приближающимися к особенностям Umbelliferae иранской флоры. Детали в распределении видов рассматривались по провинциям всех пяти стран. Список эндемичных видов для 
каждой страны был составлен на основе полевых и опубликованных данных. В Средней Азии и Казахстане насчитывается 16 эндемичных родов - Astomatopsis, Autumnalia, Fergania, Kafirnigania, Karatavia, Komarovia, Kuramosciadium, Lipskya, Mogoltavia, Paulita, Pilopleura, Schtschurowskia, Sclerotiaria, Sphaenolobium, Sphaerosciadium и Tschulaktavia. Через регион проходит важная флористическая граница между собственно ботанической Средней Азией (восточная часть ЮЗ Азиатской флористической провинции средиземноморского типа) и центральноазиатской фитохорией; первая значительно богаче зонтичными, чем вторая.

\section{Introduction}

Like the publication of the previous synopsis on nomenclature, synonymy, typification, and distribution of some Asian Umbelliferae ("Updated checklist of Chinese Umbelliferae", Pimenov, 2017, 2018), this new review has been compiled from materials stored in the GNOM and ASIUM databases (Pimenov, Leonov, 1993, 2002, 2004) at the Botanical Garden of Lomonosov Moscow State University (original software by M. W. Leonov). This review is dedicated to another important World center in of the diversity of the family taxa in Asia, namely Middle Asia and Kazakhstan. The taxonomic basis of the present publication has been updated with regard to the latest monograph of the family (Plunkett et al., 2019).

The boundaries and terminology related to the phytochorion of Middle Asia and Kazakhstan will provide some clarification, due to different interpretations in general and botanical-geographical literature. Apparently, the term "Middle Asia" appeared in Russian literature in the works of the outstanding historian S. M. Solovyev (first volume was published ad 1851), who explains this term as the area southward of the Russian Plain and eastward of the Caspian Sea. The pioneer in the use of the term in botanical literature was probably V. I. Lipsky (1900, 1904, 1910); in his "Materials ..." it is explained that Middle Asia is Russian Turkestan plus the Bukhara and Khiva khanates. After local national states became independent, the phrase "Central Asia" was often used instead of "Middle Asia". Being acceptable in a political context, these terms, however, create confusion in plant geography, since the botanical "Middle Asia" and "Central Asia" denote two considerably different contrast phytochoria (Grubov, 1959; Kamelin, 1973, 1990; Takhtajan, 1978).

In the botanico-geographical part of this publication, the most important task was to adequately and sufficiently detailed characterize the distribution of each species of the MA \& K. Umbelliferae It is much easier to register actual plant distribution with regard to administrative-territorial division (states, provinces and districts) than constantly changing borders of phytochoria in different author's inter- pretation. Therefore, species distribution is shown here by country (Kazakhstan, Tajikistan, Uzbekistan, and Turkmenistan) and its currently accepted provinces. The geographical names used are spelt as in the Times Atlas of the World: Comprehensive Edition (2011).

The characteristics by countries and provinces, compiled taking into account numerous literary, herbarium and field data, are the most accurate and pragmatic from the point of view of further use. But to this I tried to add a botanico-geographical characteristic (regardless of the administrative boundaries), but in the most general form, since there are numerous and difficult to eliminate contradictions between the data of different authors on the botanical regionalization of Asia. Here Asia is divided in total into phytochories, and for each species one or several phytochories are indicated $(+$ non-Asian continents, in case of wide distribution).

There is a series of prominent original books by R. V. Kamelin $(1973,1979,1990,1998)$ devoted to the florogenetic analysis of the natural flora of mountainous Middle Asia and adjacent territories (for example, the Altai mountainous country in a broad sense). In these works, materials on the flowering plants of wide taxonomic range are also a unique basis for clarifying particular issues of botanical geography, allow us to consider the Umbelliferae against the background of regional and often global problems of flora.

In recent posthumous publications by $\mathrm{R}$. V. Kamelin (2017, 2018), the region of Mountainous Middle Asia is included in the SW Asian province ( "Переднеазиатская область") of the Ancient Mediterranean subkingdom, while the territory of Kazakhstan covers parts of three floristic provinces two Ancient Mediterranean (SW Asian and Sahara-Gobi provinces) and one of Boreal subkingdom (the Steppe province). This is a broader viewpoint on Asian biogeography, in particular on Mediterranean (Ancien Mediterranean) phytochorion, the part of which is the greater Middle Asia. The same was the position of Meusel (1971), the Mediterranean of whom reachs foodhills of the Himalaya. Apparently, the modern understanding of the specifics of this region goes back to the ideas of the eastern Ancient 
Mediterranean and even to "flora orientalis" by P. E. Boissier.

In contrast to Chinese Umbelliferae, investigated by botanists from different countries, the Middle Asian representatives of the family were studied almost entirely within the framework of one botanical school, namely Russian or Soviet, and of its derivatives after the collapse of the Soviet Union, with little involvement of foreign scientists. Even if there were some botanists of different ethnic background, like A. Schrenk, A. Bunge, A. Lenmann etc., they lived and served in the Russian Empire, and therefore can hardly be considered foreigners.

Although 33 Umbelliferae species currently known from the territory of Middle Asia and Kazakhstan were described by Linnaeus (1753), the true regional diversity of the family began to show itself only in the first half of the 19th century, when the first original researchers with their herbaria came to the region. In particular, these were A. Lehmann, whose herbarium was treated by A. Bunge $(1843,1848,1854)$, in mountainous Middle Asia, and G. S. Karelin \& I. P. Kirilov (1841, 1842; see also Botschantzev, 1976; Gubanov et al., 1998) and independently A. Schrenk (1843); Fischer \& Meyer $(1841,1842,1845)$ in North and East Kazakhstan. For instance, A. Bunge described the new genera Hyalolaena Bunge and Eremodaucus Bunge on the basis of Lehmann's collection. The Schrenk's collection was studied not only by the botanist himself, but also by Fisher and C. A. Meyer, Regel and Schmalhausen, Trautvetter and other taxonomists.

As various parts of Middle Asia gradually joined or became associated with the Russian Empire, their botanical research intensified (Borszcszov, 1860; Regel, Herder, 1866; Osten-Saken, Ruprecht, 1869; Kaufmann, 1870, 1871; Regel et Schmalhausen, 1878a-b, 1882; Franchet, 1883; Lipsky, 1896, 1900, 1904; Fedtschenko, 1899, 1902; KosoPoljansky, 1913a-б, 1914, 1915a-б, 1916а-б, 1921, 1922, 1925). At this time, the new genera Albertia Regel et Schmalh., Angelocarpa Rupr., Euryangium Kauffm., Galagania Lipsky, Holopleura Regel et Schmalh., Korshinskia Lipsky, Kozlovia Lipsky, Ladyginia Lipsky, Renarda Regel, Schtschurowskia Regel et Schmalh., and Semenovia Regel were described from the region. The results of botanical studies, carried out in this period were summed up by B. A. Fedtschenko (Fedtschenko O. A., Fedtschenko B. A., 1909; Fedtschenko, 1915), and B. M. KosoPoljansky (1920).

It should be noted that multiple taxonomic questions regarding local genera and species of the Um- belliiferae became clearer after the publication of Boissier's "Flora Orientalis" (Boissier, 1872, 1888), which included 25 species from Middle Asia and Kazakhstan, as well as 56 species of the family described from neighboring countries and later found in Middle Asia. Nomenclature of the Umbelliferae of Boissier's Oriental herbarium, kept now in the Conservatoire et Jardin Botaniques de la ville de Genève (G-BOIS), was critically studied by Pimenov \& Jacquemoud (2020).

After the Revolution, regional research centers were set up in Middle Asia and Kazakhstan, such as universities, botanical gardens, and academic institutions with herbaria. Regional botanical schools appeared in Tashkent, Alma-Ata, Dushanbe, Ashgabat and some other cities, led by prominent scientists who came from the central USSR for a permanent job. The biggest school appeared in Tashkent with the core in the Middle Asian University. Tashkent became the regional center in Umbelliferae investigation too. Prominent botanist prof. E. P. Korovin, with broad interests in taxonomy, phylogeny, ecology, floristics, geobotany and plant morphology, since 1923 until his death in 1963 published the regional treatments of Umbelliferae from Turkmenistan (Korovin, 1950), Uzbekistan (Korovin, 1959), Kazakhstan (Korovin, 1963) and Tajikistan (Korovin et al., 1984), compiled an unique illustrated monograph of the genus Ferula (Korovin, 1947) and critically studied many other Umbelliferae genera. The treatment of Umbelliferae in "Flora of the USSR" (1950, 1951), in which the majority of the genera were processed by prof. B. K. Schischkin from the Komarov Botanical Institute of RAS, summed up the knowledge on the Umbelliferae from Middle Asia, including five new genera and 26 new species from the region.

Next new regional Umbelliferae treatment was published (Pimenov, 1983), which was a part of critical summary of the Middle Asian flora except that of the northern boreal part of Kazakhstan, without morphological descriptions and with determination keys, extensive synonymy of species, and distribution of species in the mountainous part of the territory, based on ridges rather than administrative subdivisions. The multivolume publication was conceived by Tashkent botanists under the leadership of A. I. Vvedensky, to include 15 new Umbelliferae species. Subsequent seven years were very difficult for the whole Soviet science, in particular due to severe financial limitations, especially in the national republics of Middle Asia and in Kazakhstan. Nevertheless, some scientific projects in taxonomic and 
floristic botany continued. A regional summary of Umbelliferae for Tajikistan (Korovin et al., 1984) was published, and that for Kyrgyzstan was started, to be come out later (Pimenov, Kljuykov, 2002).

The last period (2016-2020) saw the continuation of the description of new taxa from Middle Asia and Kazakhstan, against the background of profound political changes in the region, including the formation of five independent states from the former Soviet Republics, followed by dramatic events in some of them, especially in Tajikistan and Turkmenistan, which hampered or even destroyed local science. The old scientific schools largely cessed to exist. In fact, botanical activity persisted only in Tashkent, where a new floristic project, "Flora of Uzbekistan", began. Only three volumes have so far been published (2016-2019), containing the treatment of Amaryllidaceae (Khassanov, 2016), and some other families (Flora of Uzbekistan, Sennikov (ed.), 2016, 2017, 2019); treatments of some other families, including Umbelliferae (Apiaceae), are still being prepared. In the botanical garden of Moscow State University, an extensive effort in critical studies of Asian Umbelliferae based on integrative taxonomy is under way. In contrast to earlier research, these investigations, including field studies and the checking of local herbaria, involve such neighboring Asian countries, as China, Turkey, Iran, India, Nepal, and even Afghanistan, which enables one to treat Middle Asian taxa in a broader context. Herbarium collections, including type ones, kept in World herbaria, as K, P, BM, E, G, B, S, L, M, JE, etc., became available. Treatments or up-to-date listings of the Umbelliferae from neighboring countries were published, including Russia (Pimenov, Ostroumova, 2012), China (She Menglan et al., 2005; Pimenov, 20176, 2018), Kyrgyzstan (Pimenov, Kljuykov, 2002), Afghanistan (Brechle et al., 2013), Iran (Mozaffarian, 2007), Turkey (Güner et al., 2012) and others, as well as numerous articles, partly concerning the Middle Asian region. It would be impossible to enumerate all these publications here, however some of them can be found in the reference list or nomenclatural citations for a particular species.

Specially, for this publication and a separate review of the type materials, kept in TASH, the barcoding of Umbelliferae types of TASH have been carried out using HerbScan TM224 and Epson Exptession 100000XL with resolution 360dpi and 600 dpi, respecuvely under the guidance of Prof. Komiljon Tojibaev, the Director of the Institute of Botany of the Academy of Sciences of Republic of
Uzbekistan. New collections, made by botanists of this institute, for instance the interesting gatherings by Orzimat Turginov with colleagues in the mountains, arrounding Fergana, were also included. The newly accepted barcodes for broader type collection of the Umbelliferae of Middle Asia were made in St. Petersburg (LE). They are used in the present publication.

The structure of this publication is similar to that of Chinese Umbelliferae Conspectus, and provides for each taxon (species or sometimes infraspecific taxon) the accepted name, the data of the protologue, the basionym, if different from the accepted name, the type materials, synonyms (with the same data as for accepted names), and distribution by country and province, and outside the considered region by countries, as well as inclusion in large phytochoria (SW Asia, Central Asia, and so on). Barcodes of type material would be of use, however there are few of them for specific taxa of this region thus far. As of today, all herbarium materials at Moscow University (MW) and a small part of huge type collections at the Komarov Botanical Institute (LE), including Umbelliferae types of Middle Asia, have been digitized. In connection with this publication, the barcodes were recently generated (under the guidance of K. Sh. Tojibaev, the Director of the Institute of Botany of Academy of Sciences of Republic of Uzbekistan) for type material, kept in TASH, the most essential and rich herbarium collection located in the Middle Asian region.

\section{Conspectus of the Umbelliferae of Middle Asia and Kazakhstan (MA \& K)}

Aegopodium alpestre Ledeb., 1829, Fl. Altaic. (Ledebour) 1: 354.

$\equiv$ Carum alpestre (Ledeb.) Koso-Pol., 1916, Bull. Soc. Imp. Naturalistes Moscou, n. s. 29: 199.

Type: RUSSIA. [Altai] "Tscharysch, Meyer [Ledebour] 414 (268/742/918)" (lectotype LE!, designated by V. M. Vinogradova (2005: 958); isolectotypes C! [C10005768]; Circa fontes fl. Belaja Uba et Tscharysch, VII 1826, Meyer [Ledebour] (syntypes LE!, P! [P02518728, P02518744]); KAZAKHSTAN. In subumbrosis alpestribus v. gr. in latere boreali montis crucis prope Riddersk. Meyer [Ledebour] (syntype LE?).

Distribution in MA \& K: Kazakhstan (NE: E Kazakhstan prov.).

Distribution in other Asian countries: Russia, China, Japan, Korea, Mongolia.

Distribution area: Northern and Eastern Asia. 
Aegopodium kashmiricum (R. R. Stewart ex Dunn) Pimenov, 1992, Byull. Moskovsk. Obshch. Isp. Prir. Otd. Biol. 97(4): 114.

$\equiv$ Pimpinella kashmirica R. R. Stewart ex Dunn, 1924, Bull. Misc. Inform. Kew: 384.

Type: INDIA. "NW India, Sonamarg, at 3-4000 m, VII 1921, Stewart 6267" (lectotype K! [K000685615], designated by Pimenov, 2018, in Turczaninowia 21, 1: 117).

- Aegopodium alpestre auct. non Ledeb.: O. et B.

Fedtsch., 1911, Beih. Bot. Centralbl. 28(2): 30, p. p.

- Aegopodium tadshikorum auct. non Schischk.:

V. M. Vinogr. 1988, Novit. Syst. Pl. Vasc. 25: 121, p. p.

Distribution in MA \& K: Kazakhstan (SE: Almaty prov.), Kyrgyzstan (Batken prov., JalalAbad prov., Ysyk-köl prov., Osh prov., Naryn prov., Talas prov., Chüh prov.), Uzbekistan (Toshkent prov.).

Distribution in other Asian countries: China, India, Pakistan.

Distribution area: Central, South-Western and Eastern Asia.

Aegopodium podagraria L., 1753, Sp. Pl.: 265.

Type: EUROPE. "In Europa ad sepes inque pomariis, 107, Aegopodium I" (lectotype BMCliff.! [BM000558400], designated by Reduron \& Jarvis, 1993: 15; see also Reduron, 2007).

Distribution in MA \& K: Kazakhstan $(\mathrm{N}$ : Akmola prov.; NE: E Kazakhstan prov.).

Distribution in other Asian countries: Russia, Georgia, Turkey.

Distribution area: Asia (Northern), Europa, N. America, Australia \& Oceania.

Aegopodium tadshikorum Schischk., 1950, in Schischkin (ed.), Fl. URSS 16: 600, 456.

Type: TAJIKISTAN. "Asia Media, Baldzhuan, in faucibus fl. Jach-su, 04 VII 1897, Lipsky" (holotype LE! [LE01065718]).

- Aegopodium podagraria auct. non L.: O. et B. Fedtsch., 1909, Consp. Fl. Turkest. 3: 81.

Distribution in MA \& K: Kazakhstan (NE: E Kazakhstan prov.; S: S Kazakhstan prov., Zhambyl prov.; SE: Almaty prov.), Kyrgyzstan (Batken prov., Jalal-Abad prov., Ysyk-köl prov., Osh prov., Talas prov., Chüh prov.), Tajikistan (Kŭhistoni Badakhshan, Sughd prov., Khatlon prov., Dushanbe prov.), Uzbekistan (Samarqand prov., Qashqadarya prov., Toshkent prov.).

Distribution in other Asian countries: China.

Distribution area: Central and South-Western Asia.
Anethum involucratum Korovin, 1947, Not. Syst. Herb. Inst. Bot. \& Zool. Acad. Sci. Uzbekistan 8: 11.

Type: UZBEKISTAN. "Pamir-Alaj, vallis fluv. Kaschka-Darja, in regione urbis Karschi, 1928, Linczevski 273" (holotype TASH - not found).

Note: Very critical or even enigmatic species. Korovin's description does not correspond to the characteristics of the genus Anethum (Schischkin, 1951: 17), distributed in the Middle Asia only in culture, but rather to the characters of the genus Galagania. The final decision can be obtained by examining a type specimen. Schischkin (1.c.) believed that the type of this name is in Tashkent. Unfortunately, it was not found in Tashkent herbaria. The question remains open. Nevertheless, Kljuykov (2020), who does not have a type or other reliable material according to Anethum involucratum, considered it possible to transfer the species to the genus Galagania with a new nomenclature combination and put the newly described species in the synonymy of it. Until the type of Anethum involucratum has been found, all this is not very good. In the Karshi region, from where A. involucratum was described as a weed in the field Anethum tenuisectum is a fairly common species.

Angelica brevicaulis (Rupr.) B. Fedtsch., 1909, Consp. Fl. Turkest. (O. A. Fedtschenko et B. A. Fedtschenko) 3: 99; ejusd. 1911, Beih. Bot. Centralbl. 28(2): 44.

इAngelocarpa brevicaulis Rupr., 1869, Mém. Acad. Imp. Sci. Saint-Petersbourg (sér. 7) 14(4) (Sert. Tiansch.): 48.

$\equiv$ Archangelica brevicaulis (Rupr.) Rchb. f. 1876, J. Bot. 14: 46.

EColopleurum brevicaule (Rupr.) Drude, 1898, in Nat. Pflanzenfam. (Engler \& Prantl) 3, 8: 212.

Type: KYRGYZSTAN. "Tian-Schan, Suukty (Tschatyr-kol), 02 VIII 1867, Osten-Saken" (lectotype LE! [LE01065791], designated by V. M. Vinogradova (1999:81)); "In regione sylvatica jugi Thian-Schan Toyn-Thal. 02 VIII 1867, Osten-Saken" (syntype LE! [LE01065792, LE01065793]).

$=$ Archangelica decurrens Ledeb. var. alpina Regel et Herder, 1866, Bull. Soc. Imp. Naturalistes Moscou 39, 3: 77.

Type: "Thian Schan. Semenov" (holotype LE [LE01065815, LE01065816]).

= Archangelica songorica (Regel et Schmalh.) Lipsky ex Paulsen, 1903, Vidensk. Meddel. Naturhist. Foren. Kjøbenhavn 1903: 143.

= Angelica songorica Regel et Schmalh., 1878, Trudy Imp. S.-Peterburgsk. Bot. Sada 5, 2: 590. 
Type: KYRGYZSTAN. "Kok-djar-Thale im Thian-Schan-Gebirge, in einer Hohe von 8000', Osten-Saken" (syntype LE [LE010659214]).

= Angelica songorica Regel et Schmalh var. ovatifolia Regel et Schmalh. 1878, Trudy Imp. S.-Peterburgsk. Bot. Sada 5, 2: 591.

Type: KYRGYZSTAN. "In jugis montium Alexander in trajectu Kara-bura 8000' alt. A. Regel' (lectotype LE [LE01065817], designated here).

= Angelica songorica Regel et Schmalh var. rotundifolia Regel et Schmalh., 1878, Trudy Imp. S.-Peterburgsk. Bot. Sada 5, 2: 591.

Type: KYRGYZSTAN. "In Kokania inter jugo Isfairam et glacies Tantis-bai, 8-11000'alt. O. A. Fedtschenko" (lectotype LE! [LE01065813]; designated here; Kokand khanate, glacier Tschtschurovskogo. 1871. O. A. Fedtschenko (syntype LE [LE01065808]).

Distribution in MA \& K: Kazakhstan (S: S Kazakhstan prov., Zhambyl prov.; SE: Almaty prov.), Kyrgyzstan (Batken prov., Jalal-Abad prov., Ysyk-köl prov., Naryn prov., Osh prov., Talas prov., Chüh prov.), Tajikistan (Kŭhistoni Badakhshan, Sughd prov., Dushanbe prov.), Uzbekistan (Samarqand prov.?, Qashqadarya prov.).

Distribution in other Asian countries: China.

Distribution area: Central and South-Western Asia.

Angelica decurrens (Ledeb.) B. Fedtsch., 1909, Consp. Fl. Turkest. (O. A. Fedtschenko \& B. A. Fedtschenko) 3: 99.

$\equiv$ Archangelica decurrens Ledeb., 1829, Fl. Altaic. (Ledebour). 1: 316.

$\equiv$ Archangelica officinalis (Moench) Hoffm. var. decurrens (Ledeb.) Ave-Lall., 1842, in Fischer et al. (eds.), 1842, Index Sem. Horti Petrop. 9: 61.

$\equiv$ Angelica archangelica L. var. decurrens (Ledeb.) Weinert, 1973, Feddes Repert. 84(4): 309.

$\equiv$ Angelica archangelica L. subsp. decurrens (Ledeb.) V. B. Kuvaev, 1981, Bot. Zhurn. (Leningrad) 66(7): 952.

Type: KAZAKHSTAN. [Altai] "Hab. ubique ad rivulorum margines. Ad fluvium Kutschum, VI 1826, Meyer 214" (lectotype LE! [LE01071446], designated by V. M. Vinogradova 2005: 959).

Distribution in MA \& K: Kazakhstan (NE: E Kazakhstan prov.; Central: Karagandy prov.; S: S Kazakhstan prov.; SE: Almaty prov.), Kyrgyzstan (Jalal-Abad prov., Ysyk-köl prov., Naryn prov.).

Distribution in other Asian countries: Russia, China, Mongolia.

Distribution area: Asia (Arctic, Northern, Central, and South-Western), Europa (E).
Angelica komarovii (Schischk.) V. N. Tikhom., 1967, Biol. Nauki, Moscow 1(37): 91.

$\equiv$ Archangelica komarovii Schischk., 1951, in Schischkin (ed.), Fl. URSS 17: 353, 31.

$\equiv$ Angelica archangelica L. f. komarovii (Schischk.) Weinert, 1973, Feddes Repert. 84(4): 310.

Type: TAJIKISTAN. "Asia Media, Montes Zeravschanici, ad ripam lacum Iskander-kul. 18 VIII 1893. Komarov" (lectotype LE! [LE00052168]; designated here; isolectotypes A [A00076080], LE [LE01065809]).

- Angelica decurrens auct. non Ledeb.: O. et B. Fedtsch., 1909, Consp. Fl. Turkest. (O. A. Fedtschenko et B. A. Fedtschenko) 3: 99, quoad pl. pamiro-alaic.

- Archangelica tschimganica auct. non Schischk.: Korovin, 1959, Fl. Uzbekist. 4: 395, p. p.

Distribution in MA \& K: Kyrgyzstan (Batken prov., Jalal-Abad prov., Osh prov.), Tajikistan (Kŭhistoni Badakhshan, Sughd prov., Khatlon prov., Dushanbe prov.), Uzbekistan (Dzhizak prov.).

Distribution in other Asian countries: Afghanistan.

Distribution area: South-Western Asia.

Angelica multicaulis Pimenov, 1972, Byull. Moskovsk. Obshch. Isp. Prir. Otd. Biol. 77(5): 90, tab. 1, 3, map 2(2).

Type: KYRGYZSTAN. "Asia Media, Tian-Shan centralis, jugum Terskei-Alatau, in valle fluvii Sarydzhas inter ostia fluminis Kuilu et M.Taldysu, ad declivia conglomeratis, 21 VIII 1969, Pimenov, Borjaev \& Trusov 2095" (holotype LE! [LE01065810]; isotypes LE! [LE01065811], MW! [MW0593939]).

= Angelica tichomirovii V. M. Vinogr. 1986, Novit. Syst. Pl. Vasc. 23: 93, fig. 1.

Type: CHINA. "Dzhungaria, Tian-Schan, jugum Iren-Chabirga, mons Monguto, $2700 \mathrm{~m} \mathrm{s.} \mathrm{m.} 02$ VIII 1879. A. Regel" (holotype LE!).

Distribution in MA \& K: Kyrgyzstan (Ysykköl prov., Naryn prov.?).

Distribution in other Asian countries: China.

Distribution area: Central Asia.

Angelica sylvestris L., 1753, Sp. Pl.: 251.

Type: EUROPE. "In Europae frigidioris subhumidis sylvaticis, Herb. Clifford, 97, Angelica" (lectotype BM-Cliff!, designated by Reduron et Jarvis, 1992: 557; see also: Reduron, 2007).

= Taeniopetalum peucedanoides Bunge, 1852, Beitr. Fl. Russl. (Lehm. Rel. Bot.): 127.

Type: EUROPE, RUSSIA. "Auf der Linie zwischen Orenburg und Orskaja, VII 1840, Lehmann 
540" (syntypes P! [P00731602], W!); “Zwischen Ufa und Sterlitamak, 22 VIII 1840, Lehmann" (lectotype P! [P0073100, P00731691], designated by Pimenov, 2017, in Turczaninowia 20, 2: 112).

Distribution in MA \& K: Kazakhstan $(\mathrm{N}$ : Akmola prov.; NE: E Kazakhstan prov.; Central: Karagandy prov.).

Distribution in other Asian countries: Russia, China, Mongolia, Georgia, Turkey.

Distribution area: Asia (N, Central and SW), Europa, Africa, N. America (adv).

Angelica ternata Regel et Schmalh., 1878, Trudy Imp. S.-Peterburgsk. Bot. Sada 5(2): 590, tornata; 1882, Izv. Obshch. Ljubit. Estestv. 34(2) (Descr. P1. Nov. Rar. Fedtsch.): 32.

$\equiv$ Callisace ternata (Regel et Schmalh.) KosoPol., 1916, Bull. Soc. Imp. Naturalistes Moscou, n. s. 29: 179 .

Type: KYRGYZSTAN. "In Kokania, a trajectum Isfairam ad Alai, 8000' (var cordifolia) 20 VII 1871, O. A. Fedtschenko" (lectotype LE! [LE01071441], designated by V. M. Vinogradova 1999: 81; isolectotype LE [LE01071442], "Iskanderkul (var. petiolulata). O. A. Fedtschenko" (syntype LE [LE01071443-LE01071445]).

$=$ Peucedanum skardicum C. B. Clarke, 1879, in Fl. Brit. India (J. D. Hooker) 2: 711.

Type: PAKISTAN. "Baltistan, near Scardo, alt. $12000 \mathrm{ft}$ [Birjila, Kashmir, $12000 \mathrm{ft}$.], 01 VIII 1876, Clarke 29882A" (holotype K! [K000685393]).

= Angelica strattoniana Aitch. et Hemsl., 1882, J. Linn. Soc. Bot. 19: 164, tab. 13, fig. 1-7.

Type: AFGHANISTAN. "Nuristan, Mount Sikaram westwards to Mount Seratigah, growing out of the clefts of rocks from 9000 to 13000 feet, 26 VII 1880, Aitchison 394" (lectotype K!; designated by Pimenov and Jacquemoud, 2017, in Turczaninowia 20, 2: 112; isolectotypes DD!, G-BOIS!).

Distribution in MA \& K: Kyrgyzstan (Batken prov., Jalal-Abad prov., Osh prov.), Tajikistan (Kŭhistoni Badakhshan, Sughd prov., Dushanbe prov.), Uzbekistan (Samarqand prov., Qashqadarya prov., Surxondarya prov.).

Distribution in other Asian countries: China, Afghanistan.

Distribution area: Central and South-Western Asia.

Angelica tschimganica (Korovin) V. N. Tikhom., 1967, Biol. Nauki, Mosc. 1 (37): 91.

$\equiv$ Archangelica decurrens Ledeb. var. tschimganica Korovin, 1926, Byull. Sredne-Asiatsk.
Gosud. Univ. 14: 12, Suppl. (Sched. Herb. Fl. As. Med. 10): N 243.

$\equiv$ Archangelica tschimganica (Korovin) Schischk., 1951, in Schischkin (ed.), Fl. URSS 17: 32.

$\equiv$ Angelica archangelica L. f. tschimganica (Korovin) Weinert, 1973, Feddes Repert. 84(4): 309.

Type: UZBEKISTAN. "Prov. Sirdarya, distr. Taschkent ad ripas fl. Tschimganka, in regione fruticum, 10 VIII 1925, Korovin 243" (lectotype LE [LE01065818-LE01065820]; TASH! [TASH003696]; designated by Schischkin (1951: 32), isolectotypes BP!, C! [C10008314], G! [G00359721], LE!], MW! [MW0593949, MW0593941], S! [S12-21665], TASH! [[TASH003696]).

Distribution in MA \& K: Kazakhstan (S: S Kazakhstan prov., Dzhambyl prov.), Kyrgyzstan (Jalal-Abad prov.), Uzbekistan (Toshkent prov., Namangan prov.). Endemic.

Distribution area: South-Western Asia.

Note: Korovin (1926) described a species from one collection, representing a series of exsiccates, but he did not accept which specimen and in which herbarium he takes on the type (lectotype) of the new taxon. Schischkin $(1951: 32)$ was the first to indicate that the type is found in LE. Together with the description of Korovin this indication is a real lectotypification of the species.

Anthriscus cerefolium (L.) Hoffm., 1814, Gen. P1. Umbell.: 41, 47, tab. 1, fig. 21.

$\equiv$ Scandix cerefolium L., 1753, Sp. P1.: 257.

$\equiv$ Chaerophyllum cerefolium (L.) Crantz, 1767, Stirp. Austr. Fasc. 3: 70.

$\equiv$ Chaerophyllum sativum Lam., 1779, Fl. franç. 3: 438, nom. illeg. (Art. 52.1).

$\equiv$ Scandix tenuifolia Salisb., 1796, Prodr. stirp. Chap. Allerton: 166, nom. illeg. (Art. 52.1).

$\equiv$ Cerefolium sativum Besser, 1809, Prim. fl. Galiciae austriac. 1: 219.

$\equiv$ Anthriscus sativa (Lam.) Besser, 1822, Enum.

P1. (Besser) 13, nom. illeg. (Art. 52.1).

$\equiv$ Myrrhoides cerefolium (L.) Kuntze, 1891, Revis. gen. pl. 1: 268 .

$\equiv$ Selinum cerefolium (L.) E. H. L. Krause, 1904, in Deutschl. Fl. (Sturm), ed. 2 12: 73.

$\equiv$ Chaerefolium cerefolium (L.) Schinz et Thell., 1908, Vierteljahrsschr. Naturf. Ges. Zürich 53: 554.

Type: EUROPE. "In apris et arvis Europae australioris, 364.4" (lectotype LINN! [LINNHL364-4], designated by Jafri 1985: 16; see also: Spalik et Jarvis, 1989; Reduron, 2007). 
Distribution in MA \& K: Kazakhstan (S Kazakhstan prov.), Kyrgyzstan (Jalal-Abad prov.), Tajikistan (Dushanbe prov.), Uzbekistan (Toshkent prov.), Turkmenistan (Balkan prov., Akhal prov.).

Distribution in other Asian countries: Iran, Azerbaidzhan, Georgia, Armenia, Turkey, Iraq.

Distribution area: Asia (Northern and SouthWestern), Europa, Africa, N. America.

Anthriscus cerefolium (L.) Hoffm. var. trichocarpa Neilr. 1866, Aufz. Ungarn Slavon. Gefasspfl.: 221.

$\equiv$ Chaerophyllum trichospermum Schult. 1814, Oestr. Fl. (ed. 2) 1: 504, nom. illeg. (Art. 53.1), non Lam. (1783).

$\equiv$ Anthriscus trichosperma Spreng., 1820, in Syst. veg. ed. 15 bis (Roemer \& Schultes) 6: 525, nom. illeg. (Art. 52.1).

$\equiv$ Anthriscus cerefolium (L.) Hoffm. var. trichosperma Wimm. et Grab. 1827, Fl. Siles. 1: 291, 'trichospermum".

$\equiv$ Cereferolium trichospermum Besser, 1822, Enum. pl. (Besser): 44.

$\equiv$ Chaerefolium trichospermum Stankov, 1949, in Stankov et Taliev, Syst. Classif. Vasc. Pl. Eur. Russ.: 536.

Type: EUROPE, HUNGARY. "In Ungern haufigerls der vorige, Kitaibel'.

$=$ Chaerophyllum sativum M. Bieb. 1808, Fl. Taur.-Cauc. 1: 232, nom. illeg. (Art. 53.1), non Lam. (1779).

= Anthriscus cerefolium M. Bieb. 1819, Fl. Taur.Cauc. 3: 237, nom. illeg. (Art. 53.1), non Hoffm. (1814).

Type: RUSSIA/UKRAINE. "Ex Tauria et Caucaso rutheno" (lectotype LE-Bieb!, annotated by V. Byalt in LE).

= Anthriscus longirostris Bertol., 1837, Fl. Ital. (Parlatore) 3: 197.

$=$ Anthriscus cerefolium (L.) Hoffm. var. longirostris (Bertol.) Cannon, 1967, Feddes Repert. 74, 1-2: 36.

Type: EUROPE, ITALY. Ex Sardinia, Moris “.

Distribution in MA \& K: Tajikistan, Uzbekistan, Turkmenistan.

Anthriscus glacialis Lipsky, 1904, Trudy Imp. S.-Peterburgsk. Bot. Sada 23: 145.

Type: TAJIKISTAN. "Karategin, Nazarjailau, 02 VIII 1896, Lipsky" (lectotype LE! [LE00051013], designated by V. M. Vinogradova, 1997a: 95); "Systema fl. Zaravschan, trajectus Anzob, 10000', 20 VI 1870, O. A. Fedtschenko" (syn- types LE) "Margef ad fl. Jagnob. 18 VI 1892. Komarov" (syntypes LE! [LE00051013-LE00051015, LE00051017, LE00051018, LE00051021]); "Hissar Mts., fontes fl. Zarschob, 13 VI 1896, Lipsky" (syntype LE! [LE00051020]); "Karategin, Nazar-jailau, 02 VIII1896, Lipsky" (lectotype LE!, designated by M. G. Pimenov (Vinogradova, 1997a: 95); "Darvas, prope glaciem Schagasy in jugum Petri Primi, 03 VIII 1899, Lipsky" (syntype LE! [LE00051016]); "Kilimba, 09 VII 1897" Lipsky" (syntype LE! [LE00051023, LE00051024]).

Distribution in MA \& K: Kazakhstan (S Kazakhstan prov.), Kyrgyzstan (Osh prov.), Tajikistan (Kŭhistoni Badakhshan, Khatlon prov., Dushanbe prov.). Endemic.

Distribution area: Central and South-Western Asia.

Anthriscus sylvestris (L.) Hoffm., 1814, Gen. pl. Umbell.: 40, 46, tab. 1, fig. 19.

三 Chaerophyllum sylvestre L., 1753, Sp. P1.: 258.

Type: "In Europae pomariis \& cultis (lectotype LINN [LINN-HL- 365-1], designated by Spalik \& Jarvis, 1989).

=Anthriscus nemorosa (M. Bieb.) Spreng., 1813, Neue Schriften Naturf. Ges. Halle 2(1) (P1. Umb. \& Prodr.): 27.

$=$ Anthriscus aemula (Woronow) Schischk., 1950, in Schischkin (ed.), Fl. URSS 16: 129.

$=$ Anthriscus sylvestris (L.) Hoffm. subsp. aemula (Woronow) Kitam. 1962, Acta Phytotax. Geobot. 20: 195.

Type: EUROPE, RUSSIA. "Bashkiria, canton Sterlitamak, prope pag. Togus-Temir, sylvae querceto-betuletae, 12 VI 1928, Kucherovskaya 475" (lectotype LE!, designated by V. M. Vinogradova 2003: 106).

Distribution in MA \& K: Kazakhstan (Central: Karagandy prov.; S: S Kazakhstan prov., Zhambyl prov.; SE: Almaty prov.), Kyrgyzstan (Batken prov., Jalal-Abad prov., Ysyk-köl prov., Osh prov., Talas prov., Chüh prov.), Uzbekistan (Toshkent prov.), Tajikistan (Kŭhistoni Badakhshan, Khatlon prov., Dushanbe prov.), Turkmenistan (Balkan prov., Akhal prov.)

Distribution in other Asian countries: Russia, China, Japan, Korea, Nepal, India, Pakistan, Afghanistan, Iran, Azerbaidjan, Georgia, Armenia, Turkey, Iraq, Yemen, Lebanon, Syria.

Distribution area: Asia (Arctic, Northern, Central, Eastern, South-Western, and Mediterranean Asia), Europa, Africa, N. America. 
Aphanopleura capillifolia (Regel et Schmalh.) Lipsky, 1896, Bull. Acad. Imp. Sci. SaintPetersbourg (ser. 5) 4(4): 379.

$\equiv$ Pimpinella capillifolia Regel et Schmalh., 1882, Izv. Obshch. Ljubit. Estestv. 34(2) (Descr. Nov. Rar. Fedtsch.): 29.

$\equiv$ Carum capillifolium (Regel et Schmalh.) Koso-Pol., 1916, Bull. Soc. Imp. Naturalistes Moscou, n. s. 29: 199, nom. illeg. (Art. 53.1), non Kar. et Kir. (1841).

Type: UZBEKISTAN. "In Turkestaniae deserto inter fluvium Syr-Darya et Taschkent. $19 \mathrm{~V} 1871$. O. A. Fedtschenko" (lectotype LE! [LE00051682], designated by A. M. Geldykhanov 1992: 131); "Vallis fl. Zaravschan, inter Ulus et Dzham. 12 V 1869. O. A. Fedtschenko" (syntype LE! [LE01065701]; "Katta-kurgan. 08 V 1869. O. A. Fedtschenko" (syntype LE! [LE01065702]; "Karatau, ad fl. Tschujan. 06. 1876. A. Regel" (syntypes LE! [LE01065703LE01065706]).

Distribution in MA \& K: Kazakhstan (Centr.: Kzyl-Orda prov.; S: S Kazakhstan prov., Zhambyl prov.; SE: Almaty prov.), Kyrgyzstan (Batken prov., Jalal-Abad prov., Osh prov., Talas prov., Chüh prov.), Tajikistan (Kŭhistoni Badakhshan, Sughd prov., Khatlon prov., Dushanbe prov.), Uzbekistan (Karakalpak, Buxoro prov., Navoi prov., Dzhizak prov., Samarqand prov., Qashqadarya prov., Surxondarya prov., Sirdarya prov., Toshkent prov.), Turkmenistan (Akhal prov., Lebap prov., Mary prov.).

Distribution in other Asian countries: China.

Distribution area: Central and South-Western Asia.

Aphanopleura leptoclada (Aitch. et Hemsl.) Lipsky, 1896, Bull. Acad. Imp. Sci. SaintPetersbourg (ser. 5) 4(4): 377.

$\equiv$ Carum leptocladum Aitch. et Hemsl., 1888, Trans. Linn. Soc. Bot. London (2 ser.) 3(1): 66, tab. 22.

$\equiv$ Eriocycla leptoclada (Aitch. et Hemsl.) M. Hiroe, 1979, Umbelliferae World: 507, fig. 150.

Type: AFGHANISTAN. "Afghanistania, Harirud valley, 05 VI 1885, Aitchison 603” (lectotype K!; designated here; isolectotypes C! [C10008329], FI! [FI014839], G! [G00359737], GH [GH00075662], LE, P [P00834708]).

= Psammogeton glabrum Bornm. et Sint., 1909, in Consp. F1. Turkest. (O. A. Fedtschenko et B. A. Fedtschenko). 3: 117, nom. inval. (Art. 39.1).

Type: TURKMENISTAN. "Regio transcaspica: Aschabad in deserto salso. 13 VI 1900. Sintenis 500" (syntypes P [P03214055, P03214956, P03214958]).

= Aphanopleura fedtschenkoana Koso-Pol., 1922, Bot. Mater. Gerb. Glavn. Bot. Sada R.S.F.S.R. 3(18): 69. in nota, nom. altern.

= Carum aphanopleurae Koso-Pol., 1922, Bot. Mater. Gerb. Glavn. Bot. Sada R.S.F.S.R. 3(18): 70.

Type: UZBEKISTAN. "In Buchara, in valle Schirabad pr. Nauschachar, in versuris inter segetis copiosae, 24 VI 1916, Popov 130" (lectotype LE! [LE00051472], designated by V. M. Vinogradova, 1999: 83); "In Buchara, mons Chaudactau, 19 IV 1912, Neustrujev 391" (syntype LE! [LE00051473]); "In Buchara, regio arenoso, pr. Talimarek, 21 IV 1912, Neustrujev 714-717”' (syntypes LE [LE00051474, LE00051475); "Buchara, kischlak Chtai, 21 IV 1912, Schlessner 338" (syntype LE! [LE00051476]).

Distribution in MA \& K: Tajikistan (Khatlon prov., Dushanbe prov.), Uzbekistan (Buxoro prov., Dzhizak prov., Qashqadarya prov., Sirdarya prov., Toshkent prov.), Turkmenistan (Daşoguz prov., Akhal prov., Lebap prov., Mary prov.).

Distribution in other Asian countries: Pakistan, Afghanistan, Iran.

Distribution area: South-Western Asia.

Apium graveolens L., 1753, Sp. P1.: 264.

Type: EUROPE. "In Europae humectis, praesertim maritimis 374.3" (lectotype LINN [LINN-HL374-3]; designated by Tardieu-Blot 1967: 44; "Herb. Clifford, 107, Apium 1" (epitype BMCLIFF [BM000558404], designated by Reduron et Jarvis 1992: 557; see also: Reduron, 2007).

Distribution in MA \& $\mathbf{K}$ : Kazakhstan (S: S Kazakhstan prov.), Kyrgyzstan (Batken prov., Jalal-Abad prov.), Tajikistan (Sughd prov., Khatlon prov., Dushanbe prov.), Uzbekistan (Karakalpak, Dzhizak prov., Samarqand prov., Qashqadarya prov., Surxondarya prov., Toshkent prov., Namangan prov., Fergana prov.), Turkmenistan.

Distribution in other Asian countries: Russia, China, Japan, Indonesia, Malaysia, Vietnam, Myanmar, Bhutan, Nepal, India, Sri-Lanka, Pakistan, Afghanistan, Iran, Azerbadzhan, Georgia, Turkey, Iraq, Oman, Saudi Arabia, Yemen, UAE, Bahrain, Lebanon, Syria, Israel, Jordan, Egypt (Sinai), Cyprus.

Distribution area: Asia (Northern, Central, Eastern, South-Western, Mediterranean, W Tropical), Europa, Africa, N. America, S. America, Australia \& Oceania. 
Astomatopsis galiocarpa Korovin, 1948, Not. Syst. Herb. Inst. Bot. Zool. Acad. Sci. Uzbekistan 12: 30 .

$\equiv$ Astoma galiocarpum (Korovin) Pimenov et Kljuykov, 1981, Bot. Zhurn. (Leningrad) 66(4): 478.

$\equiv$ Astomaea galiocarpa (Korovin) Pimenov et Kljuykov, 1983, in Vvedensky (ed.), Opred. Rast. Sred. Azii 7: 194.

Type: UZBEKISTAN. "Montes Hissarici, vall. fl. Tupalang, ad trajectum Gova, 3800 m s. m., 14 VIII 1937, Lepeschkin \&t Kutaleva 17" (holotype TASH! [TASH002184]).

Distribution in MA \& K: Tajikistan (Kŭhistoni Badakhshan, Khatlon prov., Dushanbe prov.), Uzbekistan (Surxondarya prov.). Endemic.

Distribution area: South-Western Asia.

Aulacospermum alaicum Pimenov et Kljuykov, 1983, Byull. Moskovsk. Obshch. Isp. Prir. Otd. Biol. 88(6): 90, fig. 1 (D-Z).

Type: KYRGYZSTAN. "Jugum Alaicum, declivium septentrionale, in systemate fluvii Soch, pars superior faucium Augul, ad declivitates schistoses, in Juniperetum, 13 VII 1981, Pimenov, Kljuykov \& Vassiljeva 300" (holotype MW! [MW0593758, MW0593759]; isotypes B! [B 10 0278973], E! [E00000240], GB!, LE! [LE00051271], P! [P00602130], TASH! [TASH002222]).

Distribution in MA \& K: Kyrgyzstan (Batken prov.). Endemic.

Distribution area: South-Western Asia.

Note: Tashkent type collection contains only a duplicate of holotype, kept in Moscow (MW).

Aulacospermum anomalum (Ledeb.) Ledeb., 1833, Fl. Altaic. (Ledebour). 4: 335.

$\equiv$ Cnidium anomalum Ledeb., 1829, Fl. Altaic. (Ledebour). 1: 330.

$\equiv$ Pleurospermum anomalum (Ledeb.)

Fedtsch., 1915, Rastit. Turkest.: 604.

Type: RUSSIA. [Altai] In pratis fertilibus humidiusculis in convallibus fluviorum Tscharysch, Koksun, Ledebour (syntypes H! [H1132430], LE! [LE01065745], P!; KAZAKHSTAN [Altai] "Prope redout Alexandrovsk, 20 VII 1826, Meyer 243" (lectotype LE! [LE01066099], designated by V. M. Vinogradova 2005: 961; isolectotypes LE! [LE01065741, LE01066100], P!).

= Cnidium anomalum Ledeb. var. acaulis Ledeb., 1829, Fl. Altaic. (Ledebour). 1: 330.

Type: RUSSIA. "In alpe ad fontes fl. Tschegan. [In summa alpe Tscheganensi], 1826, Bunge" (holotype LE!)
= Aulacospermum schischkinii V. M. Vinogr. 2002, Bot. Zhurn. (St. Perersburg) 87(2): 141, fig. 1, 2A, map 3,2.

Type: KAZAKHSTAN. "Kazachstania, area monticulosa Centrali-Kazachstanica, prov. Karagandy, $70 \mathrm{~km}$ ad austro-orientem a urb. Karkaralinsk, mons Koschubai, 1559 m s. m. (systema montium Kent), 19 VII 1968, Karamyscheva, Vlasov \& Raczkowskaja 814" (holotype LE! [LE00050232, LE00050233]).

Distribution in MA \& K: Kazakhstan (NE: E Kazakhstan prov.; Central: Karagandy prov.; SE: Almaty prov.).

Distribution in other Asian countries: Russia, China, Mongolia.

Distribution area: Northern and Central Asia.

Aulacospermum gonocaulum Popov, 1935, Byull. Moskovsk. Obshch. Isp. Prir. n. s. 44(2): 129.

Type: KAZAKHSTAN. "Jugum Transili Alatau, declivum dextrum rupestre rivulum Alma-Atinka minoris, infra Medeo, inter arbores et frutices, 18 VI 1933, Popov" (lectotype MW! ; designated by Pimenov, 2017, in Turczaninowia 20, 2: 115; isolectotype AA!).

Distribution in MA \& K: Kazakhstan ( $\mathrm{S}$ : Zhambyl prov.; SE: Almaty prov.), Kyrgyzstan (Chüh prov.).

Distribution in other Asian countries: China.

Distribution area: Central and South-Western Asia.

Aulacospermum gracile Pimenov et Kljuykov, 1983, Byull. Moskovsk. Obshch. Isp. Prir. Otd. Biol. 88(6): 89, fig. 1 (A-G).

Type: KYRGYZSTAN. "Jugum Alaicum, declivium septentrionale, in valle fluvii Kirghiz-ata, inter pag. Aldyke et Koldai. 17 VII 1981. Kljuykov, Baranova \& Lavrova 413" (holotype MW! [MW0593760]; isotypes B! [B 10 0278972], BM! [BM000944696], E! [E00000241, E00000966], JE! [JE00019650], LE! [LE00051272], P! [P00602131], TASH! [TASH002223]).

Distribution in MA \& $\mathbf{K}$ : Kyrgyzstan (Osh prov.). Endemic.

Distribution area: South-Western Asia.

Note: Tashkent type collection contains only a duplicate of holotype, kept in Moscow (MW).

Aulacospermum ikonnikovii Kamelin, 2000, Novit. Syst. Pl. Vasc. 32: 131.

Type: TAJIKISTAN "Tajikistania, Regio Badachschania, jugum Jazgulemicum, in valle fl. 
Bartang, in parietibus abruptis faucium Spondzh (Sipanszh), alt. eo 3000 m, 08 VII 1991, Navruzschoev" (holotype LE!; isotype KHOR).

Distribution in MA \& K: Tajikistan (Kŭhistoni Badakhshan). Endemic.

Distribution area: South-Western Asia.

Aulacospermum plicatum Pimenov et Kljuykov, 1990, Bot. Zhurn. (Leningrad) 75(1): 90, fig. 1, 2.

Type: KYRGYZSTAN. "Prov. Naryn, in valle fl. Arpa infra ostium fluminis Chilbili, in declivibus aridibus schistoso-lapidosis in regione Juniperetorum, 05 VIII 1987, Pimenov \& Kljuykov 732" (holotype LE! [LE00051275]; isotypes FRU!, MW! [MW0593768-MW0593772]).

Distribution in MA \& K: Kyrgyzstan (Naryn prov.). Endemic.

Distribution area: Central Asia.

Aulacospermum popovii (Korovin) Kljuykov, Pimenov et V. N. Tikhom., 1976, Byull. Moskovsk. Obshch. Isp. Prir. Otd. Biol. 81(4): 82, fig. 4(8).

$\equiv$ Trachydium popovii Korovin, 1924, Bot. Mater. Gerb. Glavn. Bot. Sada R.S.F.S.R. 5(6): 78.

$\equiv$ Selinum popovii (Korovin) Schischk., 1950, in Schischkin (ed.), Fl. URSS 16: 563.

Type: UZBEKISTAN. "Mont. Thian-Schan in valle Ugam, in rupibus [Toshkent distr., Chumsan, montes Naudam], 15 VIII 1920, Popov 1401" (lectotype LE! [LE00051319]; designated by Korovin (1924a: 78) and Schischkin (1950: 563); isolectotypes TASH! (1380, 1400, 1402, 1406, 1407) [TASH002234-TASH002237, TASH003701]).

Distribution in MA \& K: Kyrgyzstan (JalalAbad prov.), Uzbekistan (Toshkent prov.). Endemic.

Distribution area: South-Western Asia.

Aulacospermum roseum Korovin, 1948, Not. Syst. Herb. Inst. Bot. Zool. Acad. Sci. Uzbekistan 12: 18.

Type: UZBEKISTAN. "Pamir-Alaj occidentalis, montes Chodsha gurgur-ata, in caementis ad trajectum Bel-auty, 27 VII 1934, Butkov 337" (holotype TASH! [TASH002226]).

= Aulacospermum coloratum Korovin, 1975, Izv. Akad. Nauk Tadzh. SSR, Otd. Estest. Nauk 3(60): 3.

Type: TAJIKISTAN. "Ad declivitatem borealem jugi Karategin, $15-17 \mathrm{~km}$ occidentem versus ab Obigarm, 19 VIII 1962, Strizhova 2330" (holotype TAD!).

Distribution in MA \& K: Kazakhstan (S: S Kazakhstan prov.), Kyrgyzstan (Batken prov., Jalal-Abad prov., Osh prov.), Tajikistan (Kŭhistoni
Badakhshan, Sughd prov., Khatlon prov., Dushanbe prov.), Uzbekistan (Dzhizak prov., Qashqadarya prov., Surxondarya prov., Toshkent prov.). Endemic.

Distribution area: South-Western Asia.

Note: Initially, Korovin (1948) assumed that the species is endemic to the Western Tianshan, but now it is indicated for both the W Tienshan and the W Pamiroalai.

Aulacospermum simplex Rupr., 1869, Mém. Acad. Imp. Sci. Saint-Petersbourg (sér. 7) 14(4) (Sert. Tianschan.): 49.

$\equiv$ Pleurospermum simplex (Rupr.) Drude, 1898, in Nat. Pflanzenfam. (Engler et Prantl) 3(8): 172 (cum auct. Benth. \& Hook. f.).

Type: KYRGYZSTAN. "Tian-Schan, Taschrobat-Schlucht, 04 VIII 1867, Osten-Saken" (lectotype LE!; designated by Kljuykov et al., 1976: 63).

$=$ Albertia commutata Regel et Schmalh., 1878, Trudy Imp. S.-Peterburgsk. Bot. Sada 5(2): 604.

= Trachydium commutatum (Regel et Schmalh.) M. Hiroe, 1979, Umbelliferae World: 713.

Type: KAZAKHSTAN. "In montibus alatavicis cisiliensibus, Karelin 392" (syntypes LE! [LE00051297, LE00051298]); "In montium alatavicorum jugo Kurmety et Keskelen, Semenov" (syntypes LE [LE00051299, LE00051300, LE00051301]); "In jugo Ketmen montium Thian-Schan, Kuschakevicz" (lectotype LE! [LE00051304], designated by V. M. Vinogradova 1999: 81); isolectotype LE [LE00051303]; KYRGYZSTAN. "In Kokaniae montium jugo Karakasuk et Kawuk, O. A. Fedtschenko" (syntype LE! [LE00051302]).

= Aulacospermum rupestre Popov, 1935, Byull. Moskovsk. Obshch. Isp. Prir. n. s. 44(2): 130.

= Pleurospermum rupestre (Popov) Fu Kuntsun et Ho Yehchi, 1979, in Fl. Reipubl. Popularis Sin. 55(1): 163.

Type: KAZAKHSTAN. "In ripa dextra fluminis Alma-Atinka minor infra Medeo. 23 VI 1933. Popov" (lectotype LE! [LE00051273]; designated by V. P. Goloskokov (1963: 52: 96); isolectotype AA!).

Distribution in MA \& K: Kazakhstan (S: S Kazakhstan prov., Zhambyl prov.; SE: Almaty prov.), Kyrgyzstan (Jalal-Abad prov., Ysyk-köl prov., Naryn prov., Osh prov., Talas prov., Chüh prov.), Tajikistan (Sughd prov.).

Distribution in other Asian countries: China.

Distribution area: Central and South-Western Asia. 
Aulacospermum stylosum (C. B. Clarke) Rech. f. et Riedl, 1963, in Biol. Skr. 13(4) (Symb. Afghan. 5): 28.

$\equiv$ Pleurospermum stylosum C. B. Clarke, 1879, in Fl. Brit. India (J. D. Hooker) 2: 704.

Type: INDIA. "Lahul, 11-12000 ft., Jaeschke" (syntype K! [K000685369]); "Kashmir, Falconer 3150" (lectotype K! [K000685370], designated by Pimenov, 2017, in Turczaninowia 20, 2: 116); isolectotype $\mathrm{K}$ [K000685371]).

= Hymenolaena darwasica Lipsky, 1900, Trudy Imp. S.-Peterburgsk. Bot. Sada 18(1): 72.

$=$ Pleurospermum darwasicum (Lipsky) B.

Fedtsch., 1915, Rastit. Turkest.: 604.

$=$ Aulacospermum darwasicum (Lipsky) Schischk., 1950, in Schischkin (ed.), Fl. URSS 16: 243, tab. 15, fig. 14.

Type: TAJIKISTAN. "Buchara, Darwas, jugum Masar, ad marginem glac. Abdul-Gasan, 23 VII 1899, Lipsky 184" (syntypes LE! [LE00051285, LE00051286]; "Buchara, Darwas, Gulbeda, 11400', 15 VII 1899, Lipsky 183" (lectotype LE! [LE00051283], designated by V. M. Vinogradova 1997b: 54; isolectotype LE [LE00051284]).

Distribution in MA \& K: Tajikistan (Kŭhistoni Badakhshan, Dushanbe prov.).

Distribution in other Asian countries: China, India, Pakistan, Afghanistan.

Distribution area: Central and South-Western Asia.

Aulacospermum tenuisectum Korovin, 1948, Not. Syst. Herb. Inst. Bot. Zool. Acad. Sci. Uzbekistan 12: 16.

Type: KYRGYZSTAN. "Tian-Schan centralis, in valle fl. Tachtalyk (syst. fl. Naryn), 25 VII 1927, Korovin 882" (holotype TASH! [TASH002227]; “ibid, Korovin 883" (isotype TASH [TASH002228]).

Distribution in MA \& K: Kyrgyzstan (JalalAbad prov.). Endemic.

Distribution area: South-Western Asia.

Aulacospermum tianschanicum (Korovin) C. Norman, 1938, J. Bot. 76: 233.

$\equiv$ Trachydium tianschanicum Korovin, 1924, Byull. Sredne-Asiatsk. Gosud. Univ. 7: 23. [Sched. Herb. Fl. As. Med. 1-2: N 29].

Type: KAZAKHSTAN. "Prov. Syr-Darja, distr. Aulie-ata, in montibus Alexandri [Kirghiz Alatau], prope Utsch-bulak, in declivibus pratensibus regionis subalpinae, 08 VII 1924, Mokeeva et Popov 29" (lectotype TASH! [TASH002239]; designated by Schischkin (1950: 246); isolectotypes: B!, BP!, BR
[BR0000005623839], BRNU [BRNU121486], C! [C10008339], E! [E00284198], G! [G00359801], K! [K000697396], LE! [LE00051320-LE00051322], MA [MA85999], MHA!, MO [MO-345618], MW! [MW0593778], NY [NY00406296], P! [P02422157], S! [S-G-6131], W! [W1927-0011379], $\mathrm{Z}$ [Z-000028777]).

= Silaus rubtzovii Schischk., 1950, Bot. Mater. Gerb. Bot. Inst. Komarova Akad. Nauk SSSR 13: 165.

= Silaum rubtzovii (Schischk.) Cherep., 1973, Svod Dopolneniǐ i Izmeneniı̌ k "Flore SSSR": 49.

Type: KAZAKHSTAN. "Jugum Kirghizicum, non procul $a b$ angustiis fl. Merke, in clivis septentrionalibus. Alt. 2000 m, 13 VII 1947, Rubtzov" (holotype LE! [LE01066091]).

Distribution in MA \& K: Kazakhstan (S: S Kazakhstan prov., Zhambyl prov.), Kyrgyzstan (Jalal-Abad prov., Naryn prov., Talas prov., Chüh prov.), Uzbekistan (Toshkent prov.). Endemic.

Distribution area: South-Western Asia.

Note: Schischkin (1950: 246) clarified that the type (lectotype) of A. tianschanicum is in Tashkent, i.e. in modern TASH.

Aulacospermum turkestanicum (Franch.) Schischk., 1950, in Schischkin (ed.), Fl. URSS 16: 244.

$\equiv$ Pleurospermum turkestanicum Franch., 1883, Ann. Sci. Nat. Bot. sér. 6, 16: 295.

$\equiv$ Trachydium turkestanicum (Franch.) Lipsky ex O. et B. Fedtsch., 1909, Consp. Fl. Turkest. 3: 123.

Type: UZBEKISTAN. "Turkestan, Tourpag Bell, 23 VIII 1881, Capus 518” (syntypes P! [P00834552, P00834553]; "Turkestan, Ona Ougane, 22 X 1881, Capus 519" (lectotype P! [P00834551], designated by Pimenov et Kljuykov 2002: 70).

= Aulacospermum latipennum Pavlov, 1938, Byull. Moskovsk. Obshch.Isp. Prir. n. s. 47(1): 81.

Type: KAZAKHSTAN. "Syr-Darja distr., declivia pratensia in cacuminibus montium Machat prope angustii Kara-Balty. 09 VIII 1934. N. V. Pavlov 355" (lectotype MW! [MW0593764]; designated by V. P. Goloskokov (1963: 52); isolectotype AA!).

= Aulacospermum pratense Korovin, 1948, Not. Syst. Herb. Inst. Bot. Zool. Acad. Sci. Uzbekistan 12: 17.

Type: KAZAKHSTAN. "Tian-Schan occidentalis, valle fluvii Ugam, loco Sary-Djun, declivia stepposa, 25 VIII 1932, Lepeschkin 250" (holotype TASH! [TASH002224]; M. Tschimgan Major. 29 VII 1925. Korovin 196 (syntype TASH). 
Distribution in MA \& K: Kazakhstan (S: S Kazakhstan prov.), Kyrgyzstan (Jalal-Abad prov.), Uzbekistan (Toshkent prov.). Endemic.

Distribution area: South-Western Asia.

Autumnalia botschantzevii Pimenov, 1989, Bot. Zhurn. (Leningrad) 74(10): 1493, fig. 1-3.

Type: KAZAKHSTAN. „Kazachstania, prov. Czimkent, Montes Karatau, declivum occidentale jugi Boroldai, vallis fr. Boroldai, Mts. Aktas, 19 IX 1987, Pimenov 875" (holotype LE!; isotypes AA!, LE!, MW! [MW0593951-MW0593957]).

Distribution in MA \& $\mathbf{K}$ : Kazakhstan ( $\mathrm{S}$ : S Kazakhstan prov.). Endemic.

Distribution area: South-Western Asia.

Autumnalia innopinata Pimenov, 1989, Bot. Zhurn. (Leningrad) 74(10): 1495, fig. 1, 3.

Type: UZBEKISTAN. "Uzbekistania, prov. Navoi, Montes Nuratau, jugum Karatau, declivum australe prope trajectus Kara-Karga, 21 IV 1986, Pimenov, Kljuykov \& Vassiljeva 96" (holotype LE!; isotypes MW! [MW0593958, MW0593959]).

Distribution in MA \& K: Uzbekistan (Navoi prov.). Endemic.

Distribution area: South-Western Asia.

Berula erecta (Huds.) Coville, 1893, Contr. U.S. Natl. Herb. 4: 115.

$\equiv$ Sium erectum Huds. 1762 (I-VI), Fl. Angl.: 103.

$\equiv$ Sium berula Gouan, 1762, F1. Monsp. (Gouan): 218.

三Sium incisum Pers. 1805, Syn. P1. (Persoon) 1: 316, nom. illeg. (Art 52.1).

三Apium berula (Gouan) Caruel, 1889, in Fl. Ital. (Parlatore) 8: 463.

三Selinum berula (Gouan) E. H. L. Krause, 1904, in Deutschl. F1. (Sturm), ed. 2 12: 32.

三 Siella erecta (Huds.) Pimenov, 1978, Bot. Zhurn. (Leningrad) 63(12): 1746.

Type: "Sium majus angustifolium. The lesser water Parsnep: p. 256 in Gerard, 1636, The Herball, or Generall histoire of plants" (lectotype, designated by Reduron 2007: 488).

ESium angustifolium L., 1762 (VII-IX), Sp. Pl. (ed. 2) 2: 1672.

$\equiv$ Berula angustifolia Mert. et W. D. J. Koch, 1826, in Deutschl. Fl. (Mertens et W. D. J. Koch), ed. 3 2: 433, nom. illeg. (Art. 52.1).

EBerla monspeliensium Bubani, 1900, Fl. Pyren. (Bubani) 2: 357.
Type: EUROPE. "In Europae australis aquosis nec extra aquis adscendit. [In Pyr. Catal. ad Gerona] 09 VII 1851. Bubani (?) 355.4".

= Sium erectum Huds. var. stenodon Koso-Pol., 1914, Bull. Soc. Imp. Naturalistes Moscou 28: 177.

Type: IRAN. "Persia australis, prope u. Schiras. V 1842. Kotschy 449" (syntypes LE [LE00015724, LE00015725].

= Berula orientalis Woronow ex Schischk. 1950, F1. URSS 16: 467.

= Sium orientale (Woronow ex Schischk.) Soó, 1965, Acta Bot. Acad. Sci. Hung. 11, 1-2: 244.

Type: IRAN. "In Shagnis, in scabuigines cires Khoi, prov. Aderbeidzan, 11 VI 1828, Szovits 437" (holotype LE!).

Distribution in MA \& K: Kazakhstan (S: S Kazakhstan prov., Zhambyl prov.; SE: Almaty prov.), Kyrgyzstan (Batken prov., Jalal-Abad prov., Osh prov., Talas prov., Chüh prov.), Tajikistan (Sughd prov., Khatlon prov., Dushanbe prov.), Uzbekistan (Navoi prov., Dzhizak prov., Samarqand prov., Qashqadarya prov., Surxondarya prov., Toshkent prov.), Turkmenistan (Balkan prov., Akhal prov., Lebap prov., Mary prov.).

Distribution in other Asian countries: China, India, Pakistan, Afghanistan, Iran, Azerbaidzhan, Georgia, Armenia, Turkey, Iraq, Saudi Arabia, Yeman, Syria, Israel, Jordan.

Distribution area: Asia (Northern, Eastern, South-Western, Mediterranean), Europa, Africa, N. America, Australia \& Oceania.

Bifora testiculata (L.) Spreng., 1820, in Syst. Veg. ed. 15 bis (Roemer et Schultes) 6: 38, 448.

$\equiv$ Coriandrum testiculatum L., 1753, Sp. Pl.: 256.

Type: EUROPE. "Europae australis agris, 363.2" (lectotype LINN [LINN-HL363-2], designated by Jafri 1985: 27; see also: Reduron, 2007).

Distribution in MA \& $\mathbf{K}$ : Uzbekistan (Samarqand prov.).

Distribution in other Asian countries: Iran, Azerbaidzhan, Turkey, Iraq, Saudi Arabia, Lebanon, Syria, Israel, Jordan, Cyprus.

Distribution area: Asia (Northern, SouthWestern, Mediterranean), Europa, Africa, N. America (adv.).

Bupleurum aitchisonii (Boiss.) H. Wolff, 1910, in Pflanzenr. (Engler) 43(IV, 228): 142.

三 Bupleurum olympicum Boiss. var. aitchisonii Boiss., 1888, F1. Orient. (Boissier) Suppl.: 252. 
Type: AFGHANISTAN. "Kurram valley, Afghanistan. Amongst stones at 12000 to 14000 feet, Sikaram, VIII 1878, Aitchison 929" (lectotype G - BOIS!; designated by Pimenov et Jacquemoud, 2017, in Turczaninowia 20, 2: 116; isolectotypes BM! [BM000885377], C! [C10008345], DD!, GH! [GH00075432], K!, LE!, P! [P03378462]).

Distribution in MA \& K: Tajikistan (Kŭhistoni Badakhshan).

Distribution in other Asian countries: China, India, Pakistan, Afghanistan.

Distribution area: Central Asia.

Bupleurum bicaule Helm,1809, Mém. Soc. Imp. Naturalistes Moscou 2: 108, tab. 8.

$\equiv$ Bupleurum falcatum L. var. bicaule (Helm) $\mathrm{H}$. Wolff, 1910, in Pflanzenr. (Engler) 43(IV, 228): 140.

$\equiv$ Bupleurum falcatum L. subsp. bicaule (Helm) Koso-Pol., 1915, Trudy Imp. S.-Peterburgsk. Bot. Sada 30(2) (Epitome Bupleur. Ross.): 217.

Type: RUSSIA. "In rupibus montibus prope Krasnojarsk, Helm" (holotype MW - destr.).

$=$ Bupleurum baldense Turra var. multicaule Ledeb., 1829, Fl. Altaic. (Ledebour) 1: 350.

= Bupleurum exaltatum M. Bieb. var. multicaule (Ledeb.) Ledeb., 1844, Fl. Ross. (Ledebour) 2: 266, p. p.

Type: RUSSIA. [Altai] "In locis subsalsis deserti editi ad fl. Tschuja, 1826, Bunge 224b" (holotype LE!; designated by Pimenov, 2017, in Turczaninowia 20, 2: 117; isolectotype P! [P03378451]).

= Bupleurum pusillum Krylov 1903, Trudy Imp. S.-Peterburgsk. Bot. Sada 21(1): 18.

= Bupleurum bicaule Helm var. pusillum (Krylov) Gubanov, 1983, Fl. Vostoch. Khangaya: 143.

Type: RUSSIA. "In montibus Altaicis orientalibus, locis sterilimis deserti Tschujensis, ad fl. Tschegan-Usun, Dshölo etc., nec in Mongolia boreali - in declivibus australibus jugi Tannu-Ola ad fl. Ak-karassuk [Altai, inter flumina Chegan-Usun et Elangash, in collibus continuis erraticis, 02 VII 1898, Saposhnikov" (lectotype TK!, designated by A. V. Polozhij et V. F. Balaschova 1989: 26; isolectotype LE!); "Altai, vallis fl. Chegan-Uzun infra ostium fl. Dshölo. 06 VII 1901. Krylov" (syntype LE!); "Altai, trajectus in fontes fluminis Dshölo. 29 VI 1897, Saposhnikov" (syntype TK).

Distribution in MA \& K: Kazakhstan (N: Pavlodar prov.; NE: E Kazakhstan prov.; Central: Karagandy prov.).

Distribution in other Asian countries: Russia, China, Mongolia.
Distribution area: Northern, Central and Eastern Asia.

Bupleurum densiflorum Rupr., 1869, Mém. Acad. Imp. Sci. Saint-Petersbourg (sér. 7) 14(4) (Sert. Tiansch.): 47.

三Bupleurum falcatum L. var. densiflorum (Rupr.) Koso-Pol., 1915, Trudy Imp. S.-Peterburgsk. Bot. Sada 30(2) (Epitome Bupleur. Ross.): 222.

Type: KYRGYZSTAN. "In regione alpina jugi Thian-Schan, am Tschatyrkol, 26 VII 1867, OstenSaken" (lectotype LE! [LE00051420], designated by I.A. Linczevski 1950: 304).

= Bupleurum kokanicum Regel et Schmalh., 1882, Izv. Obshch. Ljubit. Estestv. 34(2) (Descr. Pl. Nov. Rar. Fedtsch.): 29.

Type: KYRGYZSTAN. "In Kokaniae trajectu Dschiptyk 9-12000' alt., 22 VI 1871, O. A. Fedtschenko" (lectotype LE! [LE00051429], designated by I. A. Linczevski (Vinogradova, 1997a: 98).

= Bupleurum mongolicum V. M. Vinogr. 1985, Novit. Syst. P1. Vasc. 22: 194.

Type: MONGOLIA. "Dzhungaria, $110 \mathrm{~km} \mathrm{ad}$ meridiem et austro occidentem a distr. Bulgan, jugum Baitag-Bogdo, declive generele boreale, 2650 m s. m., steppa caricoso-petrophyto-varieherbosofestucosa, 08 VIII 1977, Volkova \& Raczkovskaya 7283" (holotype LE!).

Distribution in MA \& K: Kazakhstan (SE: Almaty prov.), Kyrgyzstan (Batken prov., JalalAbad prov., Ysyk-köl prov., Naryn prov., Osh prov., Talas prov., Chüh prov.), Tajikistan (Dushanbe prov.).

Distribution in other Asian countries: China

Distribution area: Central and South-Western Asia.

Bupleurum exaltatum M. Bieb. 1798, Beschr. Casp.: 156; 1800, Tab. Prov. Casp.: 113.

$\equiv$ Buprestis exaltata (M. Bieb.) Spreng., 1812, Mag. Neuersten Entdesk. Gesammten Naturk. Ges. Naturf. Freude Berlin 6: 259.

$\equiv$ Bupleurum falcatum L. subsp. exaltatum (M. Bieb.) Rouy et Camus ex Briq. 1897, Monogr. Bupleurum Alpes Marit.: 94, p. p.

$\equiv$ Bupleurum falcatum L. var. enexaltatum H. Wolff, 1910, in Pflanzenr. (Engler) 43(IV, 228): 134, nom. illeg. (Art. 52.1).

Type: "In pratis maritimis et ad latere collium, in Tauria, quam in promontoriis Caucasico minime rarum, Marchall von Bieberstein" (lectotype LE; designated by Pimenov in Bot. Zhurn. 101(10): 1225. 2016; isolectotypes B-Willd! [B-W 05647-01 0, B-W 05647-02 0], HAL [HAL0117291]). 
= Bupleurum graminifolium Georgi, 1800, Beschr. Russ. Reich 3, 4: 828, nom. illeg., non Vahl. (1794), nec Salisb. (1796).

= Bupleurum sibthorpianum Sm., 1806, in Fl. Graec. Prodr. (Sibthorp) 1: 179.

Type: EUROPE, GREECE." In Peloponesso, Sibthorp" (holotype OXF!).

= Bupleurum linearifolium DC., 1830, Prodr. (A. P. de Candolle) 4: 131.

= Bupleurum exaltatum M. Bieb. var. linearifolium (DC.) Boiss., 1844, Ann. Sci. Nat. sér. 3, 1: 150.

= Bupleurum falcatum L. var. linearifolium (DC.) Boiss. ex H. Wolff, 1910, in Pflanzenr. (Engler) 43(IV, 228): 135.

Type: IRAN. "In argillosis montium circa Badalan, distr. Khoi, prov. Aderbeidzhan Persiae, 20 VII 1828, Szovits 560" (lectotype G-DC!; designated by Pimenov, 2017, in Turczaninowia 20, 2: 120; isolectotypes JE [JE00022328], LE!).

= Bupleurum cernuum Ten. 1829, Fl. Napol. 3: 270, tab. 125.

= Bupleurum falcatum L. subsp. cernuum (Ten.) Arcangeli, 1894, Comp. Fl. Ital. (ed. 2): 590.

= Bupleurum falcatum L. var. cernuum (Ten.) $\mathrm{H}$. Wolff, 1910, in Pflanzenr. (Engler) 43(IV, 228): 136.

Type: EUROPE, ITALY. 'E comune ne' monti di Abruzzo e del Sannio; alla Manella. al Morrone, a monte Corno, a Rocca Valle oscura, alla difesa di Pettorano, a Monte carpaco, a Monte di Mezza ec. [In Aprutii] Tenore 184?", lectotype MPU [MPU019747], syntype FI!).

= Bupleurum schimperi Boiss., 1844, Ann. Sci. Nat. sér. 3, 1: 151.

$=$ Bupleurum linearifolium DC. var. schimperi (Boiss.) Boiss., 1872, Fl. Orient. (Boissier) 2: 850, "schimperianum".

Type: EGYPT (SINAI/ JORDAN (hist. Arabia petraea) "In Arabiâ petraeâ. [In rupestribus regionis Raphidim Arabiae petr.]. 25 IV 1835. Schimper 290 [299]" (lectotype G-BOIS!; designated by Pimenov et Jacquemoud, 2017, in Turczaninowia 20, 2: 120; isolectotypes A [A00075439], E! [E00000327E00000330], FI! [FI014835], G! [G00367656, G00367659, G00367676], GH [GH00075440], GOET!, HAL [HAL0026839, HAL0120829, HAL0120879], HBG [HBG512899, HBG512908, HBG512909, HBG512210, HBG512911], JE! [JE00003950], K! [K000687140, K000687144, K000687145, K000687146], LE!, MPU!, P! [P00752897-P00752899, P00752903, P00752904], S! [S-G-9394], STU [STU000046, STU000047, STU2017/1152], TUB! [TUB3114, TUB3115].
= Bupleurum kotschyanum Boiss., 1849, Diagn. P1. Orient.ser. 1, 10: 29.

= Bupleurum linearifolium DC. var. kotschyanum (Boiss.) Boiss., 1872, Fl. Orient. (Boissier) 2: 850.

Type: IRAN. "In regione inferiori montium Elbrus [Elburs] et Demawend Persiae borealis] Ad radices m. Demawend pr. p. Lar, 04 VIII 1843, Kotschy 627" (lectotype G-BOIS!: designated by Pimenov et Jacquemoud, 2017, Turczaninowia 20, 2: 120; id., Phytotaxa 466(1): 14; isolectotypes JE! [JE00006896], P! [P00752900, P00752901, P00752902], WAG [WAG0004961]); "In m. Elburs ("Elbrus"), pr. pagum Passgala, 1843, Kotschy 649" (syntypes G-BOIS!).

= Bupleurum cuspidatum Bunge, 1852, in Beitr. Fl. Russl. (Lehm. Rel. Bot.): 123; 1854, Mém. Acad. Imp. Sci. Saint-Petersbourg Divers Savants 7: 299.

Type: UZBEKISTAN. "An Felsenwanden des oberen Sarafschan, 10 IX 1841, Lehmann 527" (holotype LE [LE00051417]; isotypes P! [P00502128, P02284772], LE! [LE00051416, LE00051418]); designated by V. M. Vinogradova (1999: 83).

$=$ Bupleurum irregulare Boiss. et Kotschy, 1872, in Fl. Orient. (Boissier) 2: 850.

Type: LEBANON. "In regione alpine Libani supra Edem et Dimam, alt 5500', Aucher-Eloy 3621" (syntypes BM! [BM000793386, BM000793387], G-BOIS!, MPU! [MPU018911], P! [P00752913]); "Haut Liban, à Ain-Dahub, au pied de Mar-Sémone, au-dessus de Diman, 09 VI 1864, Blanche 3222" (syntype JE [JE00022359, JE00022360]); "Fruticosa supra Eden versus Danie occupat alt. 5500 ped., 28 VII 1855, Kotschy 331" (lectotype G-BOIS!: designated by Pimenov et Jacquemoud (2020: 14); isolectotypes BM! [BM000793385], GH [GH00075437], K! [K000687143], MPU! [MPU018910], US); SYRIA. "In subalpinis superioribus prope Hadet, VII 1824, Ehrenberg 262" (syntype G-BOIS!).

Distribution in MA \& K: Kazakhstan (NE: E Kazakhstan prov.; S: S Kazakhstan prov., Zhambyl prov.; SE: Almaty prov.), Kyrgyzstan (Batken prov., Jalal-Abad prov., Ysyk-köl prov., Osh prov., Talas prov., Chüh prov.), Tajikistan (Kŭhistoni Badakhshan, Sughd prov., Khatlon prov., Dushanbe prov.) Uzbekistan (Buxoro prov., Navoi prov., Dzhizak prov., Samarqand prov., Qashqadarya prov., Surxondarya prov., Toshkend prov.), Turkmenistan (Balkan prov., Akhal prov., Lebap prov.).

Distribution in other Asian countries: China, Afghanistan, Iran, Azerbaidzhan, Georgia, Armenia, Turkey, Iraq, Saudi Arabia, Lebanon, Syria, Israel, Egypt (Synai). 
Distribution area: Asia (Northern, SouthWestern, Mediterranean), Europa, Africa.

Bupleurum exaltatum M. Bieb. var. czimganicum (Lincz.) Pimenov et Sdobnina, 1981, in S. K. Cherepanov, Sosud. Rast. SSSR: 16.

$\equiv$ Bupleurum czimganicum Lincz., 1950, in Schischkin (ed.), Fl. URSS 16: 595, 316.

Type: UZBEKISTAN. "Tjan-Schan occidentalis, Czimgan, 16 VII 1897, O. A. Fedtschenko" (holotype LE! [LE00051419]).

Distribution in MA \& K: Kazakhstan (S: S Kazakhstan prov.), Kyrgyzstan, Uzbekistan.

Bupleurum ferganense Lincz. 1973, Novit. Syst. P1. Vasc. 10: 222.

Type: KYRGYZSTAN. "Asia Media, Kyrgyzstan, montes Tian-Schan occidentalis, jugum Ferganicum, systema fl. Gava, ad latera austro-occidentalia montis Schamurad, declivia rubro-arenosa, ca. 2000 m s. m., 10 VIII 1950, Linczevski \& Roshkova 170" (holotype LE! [LE00051422]; isotypes HBG [HBG512912], HUJ!, LE [LE00051423LE00051426], MO [MO-345348], MW! [MW0593787], P! [P04357199]).

Distribution in MA \& K: Kyrgyzstan (JalalAbad prov., Osh prov.). Endemic.

Distribution area: South-Western Asia.

Bupleurum gulczense O. et B. Fedtsch., 1908, Trudy Imp. S.-Peterburgsk. Bot. Sada 28: 18.

Type: KYRGYZSTAN. "Montes Alaici, inter Gulcza et Ljangar. 15 VIII 1901. O. A. Fedtschenko \& B. A. Fedtschenko" (lectotype LE! [LE00051428], designated by Linczevski 1950: 300).

Distribution in MA \& K: Kyrgyzstan (Jalal-Abad prov., Osh prov.), Tajikistan (Dushanbe prov.).

Distribution in other Asian countries: China.

Distribution area: Central and South-Western Asia.

Bupleurum isphairamicum Pimenov, 1989, Byull. Moskovsk. Obshch. Isp. Prir. Otd. Biol. 94(2): 80, fig. 1.

Type: KYRGYZSTAN. "Kyrgyzstan, jugum Alaicum, declivum septentrionale, vallis fluvii Surmatach in systemate fluminis Isfairam-sai, conus dejectionis glareosis, 13 VII 1987, Pimenov et al. 314" (holotype MW! [MW9593790]; isotype LE! [LE00051445]).

Distribution in MA \& K: Kyrgyzstan (Batken prov., Osh prov.). Endemic.

Distribution area: South-Western Asia.
Bupleurum krylovianum Schischk., 1935, in Krylov, Fl. Sibir. Occ. 8: 2010.

$\equiv$ Bupleurum falcatum L. var. oblongifolium Trautv. 1866, Bull. Soc. Imp. Naturalistes Moscou 39(2): 319.

$\equiv$ Bupleurum falcatum L. subsp. flexuosum Koso-Pol., 1915, Trudy Imp. S.-Peterburgsk. Bot. Sada 30(2) (Epitome Bupleur. Ross.): 221, p. p., non Bupleurum flexuosum Ledeb. s. str.

Type: KAZAKHSTAN. "In montibus Tarbagatai, 25 VIII 1841, Schrenk 457" (lectotype LE! [LE00051434]; isolectotypes LE! [LE00051432, LE00051433, LE00051435-LE00051444]).

Distribution in MA \& K: Kazakhstan $(\mathrm{N}$ : Pavlodar prov.; NE: E Kazakhstan prov.; S: Zhambyl prov.; SE: Almaty prov.), Kyrgyzstan (Ysyk-köl prov., Chüh prov.).

Distribution in other Asian countries: Russia, China, Mongolia.

Distribution area: Northern, Central and SouthWestern Asia.

Bupleurum lancifolium Hornem. 1813, Hort. Bot. Hafn. 1: 267.

Type: AFRICA, EGYPT. "Missum ex horto paris. sub nomina Bupl. d'Aegypte [plants grown in Bot. Gard., Copenhagen at 1804 from seeds collected in Egypt at 1803 by Nectoux" (lectotype C [C10008355], designated by S. S. Neves ex S. \& B. Snogerup 2001: 217; isolectotype C [C10008356]).

= Bupleurum heterophyllum Link, 1821, Enum. Hort. Berol. Alt. 1: 262.

= Bupleurum protractum Hoffmanns. et Link var. heterophyllum (Link) Boiss., 1872, Fl. Orient. (Boissier) 2: 836.

= Bupleurum subovatum Link ex Spreng. var. heterophyllum (Link) H. Wolff, 1910, in Pflanzenr. (Engler) 43(IV, 228): 48.

Type: EGYPT. "In Aegypto. Culta in H. B. Berl.".

$=$ Bupleurum subovatum Link ex Spreng. f. $a b-$ breviatum H. Wolff, 1910, in Pflanzenr. (Engler) 43(IV, 228): 48.

Type: IRAQ. "Al Ta'mim: Assyria orient., in campis ad Kerkuk 26 IV 1893. Bornmüller 1230, 7142, 7143" (syntypes JE [JE00021963]; "Pl. orient. Aucher-Eloy 3622"; "It. Orient. Sintenis 311"; TURKEY. "Şanliurfa, Mesopotamia, Biredjik, in campis. 12 V 1888. Sintenis 649" (syntype JE [JE00021961]; SYRIA. "Rif Dimashq: Damas, Djebel Kairoon, versant SW, crête de rochers. 17 IV 1856. Gaillardot 1844 220" (syntype JE [JE00021964]; "Del. 
Pl. Aegypt. Bornmüller, 3157"; "Basse Egypt et Marmar. Schweinfurth 287"; "It. Cypern, Sintenis \& Rigo 330, 331"; LIBYA. "Cyrenaica, Benghasi. III 1883. 626”; EGYPT. "Deect. Pl. W Aegypt. Al Iskandararīyah, inter segetes ad campum Caeraris pr. Alexandriam. VIII-XII 1857. Samaritani 3157" (syntype JE [JE00022059]; "Fl. cyren. Benghasi, Ruhmer 144" (syntype JE [JE00021987]; "Pl. atlant. Warion 131, 131a" (syntypes).

= Bupleurum subovatum Link ex Spreng. f. oxyphyllum H. Wolff in Pflanzenr. (Engler) 43(IV, 228): 48. 1910.

Type: IRAN. "Persia australis (Farsistan) in segetibus prope Bushir 03 III 1893. Bornmüller 363" (syntypes HBG [HBG512923], JE [JE00021998]); "It. Orint. Sintenis 311"; "Pl. aegypt. Letourneux 63"; "Fl. Cyren. Petrovic 142", p. p.; "Tripolis, Fl. Atlant., Warion 131", p. p.; PORTUGAL. "P1. Maderenses. Madeira: prope Gorgulho, in arvis. IV 1865-V 1866. Mandon 122" (syntype JE [JE00021997]).

Distribution in MA \& K: Turkmenistan (Balkan prov.).

Distribution in other Asian countries: Iran, Azerbaidzhan, Turkey, Iraq, Lebanon, Syria, Jordan, Egypt (Sinai).

Distribution area: Asia (South-Western and Mediterranean), Europa, Africa, Australia \& Oceania (adv.).

Bupleurum linczevskii Pimenov et Sdobnina, 1983, in Vvedensky (ed.), Opred. Rast. Sredn. Azii 7: 371, 211.

Type: TAJIKISTAN. "Tajikistan, pars occidentalis jugi Petri Primi, in frutices declivis septentrionalis prope trajectum Kamchirak, 12 VIII 1975, Pimenov 1126" (holotype MW! [MW0593793]; isotype LE! [LE00051447, LE00051448]).

Distribution in MA \& K: Tajikistan (Kŭhistoni Badakhshan, Sughd prov., Khatlon prov., Dushanbe prov.). Endemic.

Distribution area: South-Western Asia.

Bupleurum lipskyanum (Koso-Pol.) Lincz., 1950, in Schischkin (ed.), Fl. URSS 16: 327, in obs.

$\equiv$ Bupleurum falcatum L. f. lipskyanum KosoPol., 1915, Trudy Imp. S.-Peterburgsk. Bot. Sada 30(2) (Epitome Bupleur. Ross.): 214.

Type: TAJIKISTAN. "Buchara, Darvaz, jugum Masar, declivum septentrionale, prope glaciem Sikogach, 9800', 22 VII 1899, Lipsky 3941" (holotype LE! [LE00051449], isotypes LE [LE00051450, LE00051451]).
= Bupleurum contractum Korovin, 1975, Izv. Akad. Nauk Tadzhiksk. SSR, Otd. Estest. Nauk 3(60): 4.

Type: TAJIKISTAN. "Ad declivitatem australem jugi Petri Primi, fl. Garmo, locus Digai, 24 VII 1950, Ovczinnikov, Zaprjagaeva \& Iljinskaya 768" (holotype TAD!).

Distribution in MA \& K: Kyrgyzstan (Osh prov.), Tajikistan (Kŭhistoni Badakhshan, Sughd prov., Khatlon prov., Dushanbe prov.). Endemic.

Distribution area: Central and South-Western Asia.

Bupleurum longifolium L. subsp. aureum (Fisch. ex Hoffm.) Soó, 1966, Acta Bot. Acad. Sci. Hung. 12(1-2): 116.

三Bupleurum aureum Fisch. ex Hoffm., 1814, Gen. Pl. Umbell.: 115.

三Diaphyllum aureum Hoffm., 1814, Gen. Pl. Umbell.: 115.

三Bupleurum longifolium L. var. aureum (Fisch. ex Hoffm.) H. Wolff, 1910, in Pflanzenr. (Engler) 43 (IV, 228): 52.

Type: "Described on the basis of plants, cultivated in BG Gorenki. Herb. Hoffmann, 2252" (lectotype MW - Hoffm! [MW0593786], designated by Gubanov 1993: 93; isolectotype LE! [LE00015701]).

Distribution in MA \& K: Kazakhstan (NE: E Kazakhstan prov.; Central: Karagandy prov.; S: Zhambyl prov.; SE: Almaty prov.), Kyrgyzstan (Jalal-Abad prov., Chüh prov.).

Distribution in other Asian countries: Russia, China, Mongolia.

Distribution area: Asia (Northern, Central), Europa.

Bupleurum multinerve DC., 1828, Mém. Soc. Phys. Genève 4: 500, nom. cons.

$\equiv$ Bupleurum ranunculoides L. var. multinerve (DC.) Koso-Pol., 1915, Trudy Imp. S.-Peterburgsk. Bot. Sada 30(2) (Epitome Bupleur. Ross.): 188.

Type: CULT. "Hortus Bot. Genev. - De graines envoyées en 1826 par M. Fischer, et qui avaient recuillies au monts Altai [ex Altai], 1828, 2581/6" (lectotype G-DC! [G00203259]; designated by Pimenov in Taxon 66(5): 1230. 2017a).

= Bupleurum striatum Steph. ex Roem. et Schult. 1820, Syst. Veg. (ed. 15) 6:367, nom. rej.

Type: RUSSIA. "In Siberia. Stephan 5631" (holotype B-Willd. [B-W 05631-01 0]).

= Bupleurum multinerve DC. var. minus DC., 1830, Prodr. (A. P. de Candolle) 4: 130. 
Type: RUSSIA. "In montibus Altaicis [Altai], 1828, Fischer" (probable holotype G-DC [G00203258]).

= Bupleurum nervosum Trev., 1831, Symb. Phytol. 1: 22.

= Bupleurum multinerve DC. var. major Ledeb., 1844, Fl. Ross. (Ledebour) 2: 265.

= Bupleurum multinerve DC. var. minor Ledeb., 1844, Fl. Ross. (Ledebour) 2: 265.

= Bupleurum longeinvolucratum Krylov, 1903, Trudy Imp. S.-Peterburgsk. Bot. Sada 21(1): 17.

Type: RUSSIA. "In jugo Tigeretzki-belok montium Altaicorum occidentalium, in declivibus saxosis, infra limitem sylvarum, 31 VII 1891, Krylov" (lectotype TK!; designated by A.V. Polozhij et V.F. Balashova (1989: 26), as "type"; isolectotype LE!).

Distribution in MA \& K: Kazakhstan (NE: E Kazakhstan prov.).

Distribution in other Asian countries: Russia, China, Mongolia.

Distribution area: Asia (Northern and Central), Europa.

Note: Chubarov (2003: 301) treated B. longeinvolucratum as a separate local microspecies.

Bupleurum pamiricum Pimenov et Kljuykov, 2015, Phytotaxa 226 (3): 249.

Type: TAJIKISTAN. "Gorno-Badakhshan Autonomous Province: the Pamirs, between Murgab and Dzhilandy, riv. Nichke-Dzhilga (tributary of Murgab river), 27 VII 1981, Pimenov, Kljuykov, Baranova, Vasilieva \& Tomkovich 892" (holotype MW! [MW0595634]; isotype MW [MW0595635]).

Distribution in MA \& K: Tajikistan (Kühistani Badakhshan).

Distribution in other Asian countries: Afghanistan.

Distribution area: Central Asia.

Bupleurum rosulare Korovin ex Pimenov et Sdobnina, 1983, in Vvedensky (ed.), Opred. Rast. Sred. Azii 7: 372, 211.

Type: KAZAKHSTAN. "Kazakhstania australis, jugum Alexandri, prope Merke, 20 VII 1924, Popov \& Mokeeva" (holotype TASH! [TASH002271]).

Distribution in MA \& K: Kazakhstan (S: Zhambyl prov.), Kyrgyzstan (Talas prov.). Endemic.

Distribution area: South-Western Asia.

Bupleurum rotundifolium L., 1753, Sp. P1.: 236. Type: EUROPE. "Inter Europae australis segetes, 335.1” (lectotype LINN! [LINN-HL335-1], designated by K. H. Rechinger et S. Snogerup 1987: 272).

Distribution in MA \& K: Kyrgyzstan (Batken prov.), Turkmenistan (Balkan prov., Akhal prov.)

Distribution in other Asian countries: Russia, India, Pakistan, Iran, Azerbaidzhan, Georgia, Armenia, Turkey.

Distribution area: Asia (Northern, Eastern and South-Western), Europa, Africa, N. America (adv.), Australia \& Oceania (adv.).

Bupleurum semicompositum L., 1753, Demonstr. P1.: 7.

$\equiv$ Bupleurum divaricatum Lam. var. semicompositum (L.) Lam., 1779, Fl. Franc. (Lamarck) 3: 410.

$\equiv$ Odontites semicompositus Spreng., 1812, Mag. Neuersten Entdesk. Gesammten Naturk. Ges. Naturf. Freude Berlin 6: 258.

$\equiv$ Isophyllum semicompositum C. Presl, 1826, F1. Sicul.: XXV.

Type: EUROPE, SPAIN. "Hortus Upsalensis. Hispania. Loefling 335.13" (lectotype LINN! [LINN-HL335-13], designated by C. C. Townsend 1966: 81).

= Bupleurum glaucum Robill. et Castagne ex DC., 1815, in Fl. Franc. (de Candolle et Lamarck) ed. 3, Suppl.: 515.

$=$ Odontites glaucus (Robill. et Castagne) Spreng., 1820, in Syst. Veg. ed. 15 bis (Roemer \& Schultes) 6: 383 .

= Isophyllum glaucum (Robill. et Castagne) Fourr. 1868, Ann. Soc. Linn. Lyon (sér. 2) 16: 391.

$=$ Bupleurum semicompositum L. var. glaucum (Robill. et Castagne) Paol., 1900, Fl. Ital. 2: 155.

Type: EUROPE, FRANCE. "A Mazargue et la Gineste près Marcelle; aux Sablettes près Toulon; sur les sables maritimées, à Nice, Robillard \& Castagne" (syntype MPU [MPU018909]).

Distribution in MA \& K: Turkmenistan (Akhal prov.).

Distribution in other Asian countries: Russia, Iran, Azerbaidzhan, Turkey, Iraq, Kuwait, Saudi Arabia, UAE, Bahrain, Qatar, Syria, Israel, Jordan, Egypt (Sinai).

Distribution area: Asia (South-Western, Mediterranean and $\mathrm{W}$ Tropical), Europa, Africa, Australia \& Oceania (adv.).

Bupleurum thianschanicum Freyn, 1900., Mém. Herb. Boissier 13: 23.

Type: KAZAKHSTAN. "Turkestania orientalis, Thian-Schan, in valle fluvii Bajangol regionis 
silvatica superiore, 29 VII 1896, Brotherus 753" (lectotype BRNM!; designated by Pimenov \& Sutorý (2014: 1105); isolectotypes G! [G00367640], H [H1394758]).

Distribution in MA \& K: Kazakhstan (NE: E Kazakhstan prov.; SE: Almaty prov.), Kyrgyzstan (Ysyk-köl prov., Naryn prov., Osh prov.?, Chüh prov.).

Distribution in other Asian countries: China.

Distribution area: Central and South-Western Asia.

Carum carvi L., 1753, Sp. P1.: 263.

Type: EUROPE. "In Europae borealis pratis. Herb. Clifford, 106, Carum I" (lectotype BM-Cliff [BM000558387], designated by Reduron \& Jarvis in Jarvis \& al., 1993: 30).

Distribution in MA \& K: Kazakhstan (W: W Kazakhstan prov., Aktobe prov.; N: Kostonai prov., Pavlodar prov., Akmola prov.; NE: E Kazakhstan prov.; Central: Karagandy prov.; S: S Kazakhstan prov., Zhambyl prov.; SE: Almaty prov.), Kyrgyzstan (Batken prov., Jalal-Abad prov., Ysykköl prov., Naryn prov., Osh prov., Talas prov., Chüh prov.), Tajikistan (Kŭhistani Badakhshan, Sughd prov., Khatlon prov., Dushanbe prov.), Uzbekistan (Dzhizak prov., Samarqand prov., Qashqadarya prov., Fergona prov.), Turkmenistan (Akhal prov.).

Distribution in other Asian countries: Russia, China, Myanmar, Bhutan, Nepal, India, Pakistan, Mongolia, Afghanistan, Iran, Azerbaikdzhan, Georgia, Armenia, Turkey, Iraq, Saudi Arabia, Syria.

Distribution area: Asia (Northern, Central, Eastern, South-Western), Europa, Africa, N. America (adv.), New Zealand (adv.), also widely cultivated.

Caucalis platycarpos L., 1753, Sp. P1.: 241.

Type: EUROPE. "Herb. Clifford, 91, Caucalis 3 Caucalis dauci sylvestris folio echinato magno fructu". 2. 118.1 (lectotype BM-Cliff, designated by Heywood, 1960: 11; Batarda Fernandes 1967: 402; cf. Reduron 2007: 763; Leiden, van Royen (syntype L! [L0077004]).

= Daucus lappula Weber ex F. H. Wigg. 1780, Prim. Fl. Holsat.: 23, nom. illeg. (Art. 52.1).

= Caucalis lappula (Weber ex F. H. Wigg.) Grande, 1918, Bull. Ort. Bot. Napoli 5: 194.

= Caucalis daucoides L., 1767, Syst. Nat., ed 12 2: 20, nom. illeg. (Art. 53.1), non L. (1753).

Distribution in MA \& K: Kazakhstan (SE: Almaty prov.), Kyrgyzstan (Jalal-Abad prov.),
Uzbekistan (Fergona prov.) Turkmenistan (Balkan prov., Akhal prov.).

Distribution in other Asian countries: Russia, Afghanistan, Iran, Azerbaidzhan, Georgia, Armenia, Turkey, Iraq, Cyprus.

Distribution area: Asia (Northern, South-Western and Mediterranean), Europa, Africa, N. America.

Cenolophium denudatum (Fisch. ex Hornem.) Tutin, 1967, Feddes Repert. 74(1-2): 31, in obs.

三Athamanta denudata Fisch. ex Hornem. 1819, Hort. Hafn. Suppl.: 32.

三Selinum cenolophium E. H. L. Krause, 1904, in Deutschl. Fl. (Sturm), ed. 2 12: 108, nom. illeg. (Art. 52.1).

Type: KAZAKHSTAN. "In Russia" (grown in hortus Hafnensis from the seeds of Altai origin: In pratis circa fl. Irtysch et Buchtorma, Ledebour \& C. A. Meyer) (lectotype C! [C10008381]; here designated; isolectotypes C! [C10008378-C10008380]).

= Crithmum mediterraneum M. Bieb. 1819, Fl. Taur.-Cauc. 3: 215.

Type: "Ex hort. Gorenk. ex seminibus Sareptanis, 1817" (LE-Bieb!, annotated by V. Byalt in LE).

= Angelica fischeri Spreng., 1820, in Syst. veg. ed. 15 bis (Roemer et Schultes) 6: 605.

= Ligusticum fischeri (Spreng.) Link, 1821, Enum. Hort. Berol. Alt. 1: 276.

$=$ Cenolophium fischeri (Spreng.) W. D. J. Koch,1824, Nova Acta Phys.-Med. Acad. Caes. Leop.-Carol. Nat. Cur. 12(1): 103.

= Cnidium fischeri (Spreng.) Spreng., 1824, Syst. Veg. (ed. 16) 1: 888.

Type: EUROPE, RUSSIA. "Ad Volgam".

= Ligusticum divaricatum Ledeb., 1824, Ind. Sem. Hort. Dorpat.: 5.

= Cnidium divaricatum (Ledeb.) Ledeb., 1829, Fl. Altaic. (Ledebour) 1: 324.

= Cenolophium divaricatum (Ledeb.) Besser, 1832, Flora 15 (2 Beibl.): 27.

Type: (probable type material): RUSSIA. "In Sibiria altaica [Ligusticum divaricatum Ledeb.! M. Fisch. nom.: Athamanta denudata altaica" (LE!)].

= Silaus longifolius Ledeb., 1829, Fl. Altaic. (Ledebour) 1: 323, nom. illeg. (Art. 52.1).

Type: KAZAKHSTAN. "In pratis circa fl. Irtysch et Buchtorma (Ledebour, Meyer)" [Ledebour 247 (lectotype LE!, P [P03237363]).

Distribution in MA \& K: Kazakhstan (W: W Kazakhstan prov., Aktobe prov.; N: Kostonai prov., 
N Kazakhstan prov., Akmola prov., Pavlodar prov.; NE: E Kazakhstan prov.; Central: Karagandy prov.; SE: Almaty prov.).

Distribution in other Asian countries: Russia, China, Mongolia.

Distribution area: Asia (Northern and Central), Europa.

Cephalopodum badachschanicum Korovin, 1973, Izv. Akad. Nauk Tadzh. SSR, Otd. Estest. Nauk 1(50): 17, fig. 5-9.

Type: TAJIKISTAN. "Jugum Darvas, pars meridionalis, in angustis Obi-Charak, 07 VII 1968, Koczkareva \& Abdusaljamova" (holotype TAD!).

Distribution in MA \& K: Tajikistan (Kŭhistani Badakhshan), Afghanistan (NE: Badakhshan).

Distribution in other Asian countries: Afgfhanistan.

Distribution area: South-Western Asia.

Cephalopodum hissaricum Pimenov, 1983, in Vvedensky (ed.), Opred. Rast. Sred. Azii 7: 374, 245.

Type: UZBEKISTAN. "Jugum Hissaricum, pars occidentalis in systematis fluminis Tupolang, NNE et NNW abrupti saxosi ripam sinistram fl. Taminda infra pag. Taminda, regio arboreo-fruticosa, 10 VII 1948, Pjataeva 887" (holotype TASH! [TASH002334]).

Distribution in MA \& $\mathbf{K}$ : Uzbekistan (Surxondarya d.). Endemic.

Distribution area: South-Western Asia.

Cervaria cervariifolia (C. A. Mey.) Pimenov, 1987, in Flora Iranica (Rechinger) 162: 452, tab. 409.

$\equiv$ Peucedanum cervariifolium C. A. Mey., 1831, Verz. Pfl. Casp. Meer. (C. A. von Meyer): 126.

Type: AZERBAIDZHAN. "In sylvis montium Talusch (alt. 400-800 hexap.). [In sylvis umbrosissimis montium altiorum provinciae Talischensis, inter pag. Akkerman et Limar], 02 VI 1830, Meyer 1105]" (lectotype LE!; designated here by Pimenov; isolectotype P! [P00731614]).

= Peucedanum sintenisii H. Wolff, 1924, Repert. Spec. Nov. Regni Veg. 20: 68.

Type: TURKMENISTAN. "Ashkhabad, Suluklu (Saratovka), ad fines Persiae in pratis subalpinis M. Messinew, Sintenis 1056" (syntypes BP!, LE! [LE00052152-LE00052154], STU).

Distribution in MA \& K: Turkmenistan (Akhal prov.).
Distribution in other Asian countries: Iran, Azerbaidzhan.

Distribution area: South-Western Asia.

Chaerophyllum bulbosum L., 1753, Sp. P1.: 258.

$\equiv$ Myrrhis bulbosa (L.) All., 1785, Fl. pedem. 2: 29.

$\equiv$ Scandix bulbosa (L.) Roth, 1788, Tent. Fl. Germ. 1: 123.

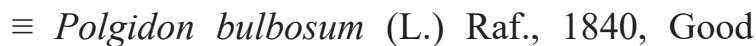
Book: 53.

$\equiv$ Selinum bulbosum (L.) E. H. L. Krause, 1904, in Deutschl. Fl. (Sturm), ed. 2 12: 49.

Type: EUROPE. "In Alsatia, Hungaria, Helvetia, 365.2" (lectotype LINN! [LINN-HL365-2]; designated by Hedge et Lamond in Rechinger 1987: 91).

= Chaerophyllum bobrovii Schischk., 1950, in Schischkin (ed.), Fl. URSS 16: 591, 115.

Type: TURKMENISTAN. "Asia media, in jugo Kopet-dagh austro-occidentali. fauces Por-dere in Juglandetis, alt. 1760 m, 24 VI 1930, Bobrov 428" (holotype LE! [LE00051008]).

Distribution in MA \& K: Kyrgyzstan (Chüh prov.), Turkmenistan (Balkan prov., Akhal prov.).

Distribution in other Asian countries: Russia, Iran, Azerbaidzhan, Georgia, Armenia, Turkey.

Distribution area: Asia (Northern, SouthWestern), Europa, N. America.

Chaerophyllum khorossanicum Czerniak. ex Schischk., 1950, in Schischkin (ed.), Fl. URSS 16: 590, 108

Type: IRAN. "Irania. Prov. Khorossan; in rupestribus inter pag. Darbendy et Kuczak, 12 VII 1925, Czerniakowskaia 100" (holotype LE! [LE00015664]; isotypes LE! [LE00015663, LE00015665, LE00015670]).

$=$ Chaerophyllum shahvaricum Rech. f., 1952, Anz. Oesterr. Akad. Wissensch. Math.-Naturwiss. K1. 89(11): 168.

Type: IRAN. "Persia: Gorgan: in declivibuis borealibus montium Shahvar inter Hajilang et Gardeneh Daht, ca. 3300 m, 27 VII 1948, Rechinger 6224" (holotype W!; isotypes E! [E00000299], G [G00367373, G00367397, G00367403], K! [K000442318]).

Distribution in MA \& K: Turkmenistan (Balkan prov., Akhal prov.).

Distribution in other Asian countries: Iran.

Distribution area: South-Western Asia. 
Chaerophyllum macrospermum (Willd. ex Spreng.) Fisch. et C. A. Mey., 1838, Bull. Soc. Imp. Naturalistes Moscou 9: 327.

三Scandix macrosperma Willd. ex Spreng., 1820, Syst. Veg. ed. 15 bis (Roemer et Schultes, eds.) 6: 507.

三Myrrhis clavata Spreng., 1824, Syst. Veg. (ed.

16) $1: 903$.

E Golenkinianthe macrosperma (Willd. ex Spreng.) Koso-Pol., 1915, Journ. Russe Bot. 1-2: 14, fig. 8.

Type: TURKEY. "Armenia, Tournrfort \& Gundelsheimer W 5898" (holotype B-Willd! [B-W 05898-01 0]).

$=$ Scandix gilanica S. G. Gmelin, 1774, Reise Russland (S. G. Gmel.) 3: 303, tab. 31, fig. 2.

= Myrrhis gilanica (S. G. Gmelin) Schult. 1820, Syst. Veg. ed. 15 bis (Roemer \& Schultes) 6: 520.

= Golenkinianthe gilanica (S. G. Gmelin) KosoPol., 1914, Trudy Bot. Sada Imp. Jur'evsk. Univ. 15: 14.

= Chaerophyllum gilanicum (S. G. Gmelin) Grossh, 1932, Fl. Kavkaza (Grossheim) 3: 126, nom. illeg. (Art. 53.1), non Chaerophyllum ghilanica Stapf et Wettst. (1886).

Type: IRAN. "Persia borealis, Gmelin".

= Grammosciadium meoides DC., 1829, Col. Mém. 5 (Mém. Fam. Ombell.): 63.

Type: "Orient, Meum orientalis semine unciam longo, Tournefort [2751]" (holotype P-TRF!).

Distribution in MA \& K: Turkmenistan (Balkan prov., Akhal prov.).

Distribution in other Asian countries: Iran, Azerbaidzhan, Armenia, Turkey, Lebanon.

Distribution area: South-Western \& Mediterranean Asia.

Chaerophyllum prescottii DC., 1830, Prodr. (A. P. de Candolle) 4: 225.

三 Anthriscus prescottii (DC.) Veesenm. 1854, Beitr. Pflanzenk. Russ. Reiches 9: 84.

$\equiv$ Chaerophyllum bulbosum L. subsp. prescottii (DC.) Nym. 1879, Consp. Fl. Eur. 2: 300.

Type: RUSSIA. "In Sibiria altaica, Schangin" (holotype G-DC).

Distribution in MA \& K: Kazakhstan (W: W Kazakhstan prov., Aktobe prov.; N: Kostonai prov., N Kazakhstan prov., Akmola prov., Pavlodar prov.; NE: E Kazakhstan prov.; Central: Karagandy prov., Kzyl-Orda prov.), Uzbekistan (Kara-kalpak).

Distribution in other Asian countries: Russia, Armenia.

Distribution area: Asia (Northern, Central), Europa.
Cicuta virosa L., 1753, Sp. P1.: 255.

三 Cicutaria aquatica Lam., 1779, Fl. franç. (Lamarck) 3: 445.

三Sium cicuta Weber ex F. H. Wigg. 1780, Prim. F1. Holsat.: 24.

$\equiv$ Cicutaria virosa Delarbre, 1800, Fl. Auvergne (ed. 2): 415 .

ESelinum virosum (L.) E. H. L. Krause, 1904, in in Deutschl. F1. (Sturm) ed. 2, 12: 44.

Type: EUROPE. "In palludibus Europae sterilibus. Ammann 361.1" (lectotype LINN! [LINN-NL 321-1], designated by Hedge et Lamond 1972: 425).

$=$ Cicuta virosa L. var. latisecta Čelak., 1875, Prodr. Fl. Boehm.: 563.

Type: EUROPE. "[Bohmen] loco non notato".

= Cicuta sachalinensis Koidz., 1930, Fl. Symb. Orient.-Asiat.: 44.

Type: RUSSIA. "Saghalin: in humidis Korsakoff. 09.1908. Faurie 384" (lectotype P!; designated by Pimenov, 2017, in Turczaninowia 20, 2: 132; isolectotype E! [E00000444]).

Distribution in in MA \& K: Kazakhstan (W: Aktobe prov.; N: Kostonai prov., N Kazakhstan prov., Akmola prov., Pavlodar prov.; NE: E Kazakhstan prov.; Central: Karagandy prov., KzylOrda prov.; SE: Almaty prov.), Kyrgyzstan (Ysykköl prov., Chüh prov.).

Distribution in other Asian countries: Russia, China, Japonia, Korea, India, Georgia, Turkey.

Distribution area: Asia (Arctic, Northern, Central, Eastern, South-Western), Europa, Australia \& Oceania (adv.).

Cnidiocarpa alaica Pimenov, 1983, Bot. Zhurn. (Leningrad) 68(1): 88, fig. B.

Type: TAJIKISTAN. "Tajikistania orientalis, declive australe jugi Alaici, prope pag. KitschikKaramyk, in loco paludoso, 24 VIII 1975, Pimenov 1332" (holotype LE! [LE01065749]; isotypes MW [MW0593930, MW0593931, MW0593932]).

Distribution in MA \& K: Kyrgyzstan (Osh prov.), Tajikistan (Dushanbe prov.). Endemic.

Distribution area: South-Western Asia.

Conioselinum tataricum Hoffm., 1816, Gen. P1. Umbell. (ed. 2): 185, fig. 5.

Type: "Described on the basis of plants, cultivated in Botanical Garden Gorenki ("hort. Gorenk.")".

= Conioselinum vaginatum Thell., 1926, in Hegi, Ill. Fl. Mitt.-Eur. 5(2): 1329, non Ligusticum vaginatum Spreng. (1815).

Type: RUSSIA. "In Sibiria". 
= Selinum gmelinii Bray, 1818, Denkschr. Bot. Ges. Regensb. 1(2): 36.

Type: EUROPE, LITHUIANIA. In fruticetis inter Corylos et alios frutices.

= Conioselinum fischeri Wimm. et Grab. 1827, F1. Siles. 1: 266.

Type: EUROPE, POLAND. "[Livonia] In montanis herbosis. Auf dem Leitenberge u. Kleinberge im Gesenke".

$=$ Conioselinum univittatum Turcz. ex Kar. et Kir. 1842, Bull. Soc. Imp. Naturalistes Moscou 15(2): 363.

= Selinum univittatum Turcz., 1844, Bull. Soc. Imp. Naturalistes Moscou 17: 735.

Type: RUSSIA. "[Baikal et Transbaicalia] In pratis sylvaticis [Ircutia], 1828, Turczaninow 117" (lectotype LE!, designated by V. M. Vinogradova 2005: 962); isolectotypes KW!, LE!).

= Conioselinum altaicum Rupr., 1859, Beitr. Pflanzenk. Russ. Reiches 11 (Revis. Umb. Kamtsch.): 22.

= Conioselinum latifolium Rupr., 1869, Mém. Acad. Imp. Sci. Saint-Petersbourg (ser. 7) 14(4) (Sert. Tiansch.): 48.

Type: KYRGYZSTAN. "In regione alpine jugi Thian-Schan, Suukty, 01 VIII 1867, Osten-Saken" (syntype LE [LE01065794]; "Molda-assu-Schlucht, 12 VIII 1867, Osten-Saken" (holotype LE! [LE01065795]; designated by V. M. Vinogradova (1999: 84).

$=$ Selinum papyraceum C. B. Clarke, 1879, in Fl. Brit. India (J. D. Hooker) 2: 701.

$=$ Cortia papyracea (C. B. Clarke) Leute, 1969, Ann. Naturhist. Mus. Wien 73: 84.

$=$ Conioselinum papyraceum (C. B. Clarke) Pimenov et Kljuykov, 1999, Bot. Zhurn. (St. Perersburg) 84(3): 91, fig. 1, map 2.

Type: INDIA. "Polgam, Kashmir, 12000 ft., 04 IX 1875, Clarke 31047" (lectotype K! [K000685150], designated by E. Nasir 1972: 117).

$=$ Conioselinum schugnanicum B. Fedtsch., 1902, Trav. Mus. Bot. Acad. Petersb. 1: 135.

Type: TAJIKISTAN. "In valle fl. Gunt prope Rivak (Rivak-Chorog), in frutices, 29 VII 1901, B. A. Fedtschenko" (lectotype LE! [LE01065796]; designated by V. M. Vinogradova 1999: 84; isolectotypes LE [LE01065799, LE01065800, LE01065801]); "A castellum Chorog ad pag. Rivak, ad riguorum rivulorum margines, in frutices, 29 VII 1901, B. A. Fedtschenko" (syntypes LE! [LE01065797, LE01065798, LE01065802]); "In valle fl. Toguz-bulak, a locum Vankala (in fl. Gunt) ad locum Dzhilandy, 21-31 VII 1901, B. A. Fedtschenko" (syntype
LE! [LE01065802, LE01065803, LE01065804]).

= Vvedenskya pinnatifolia Korovin, 1947, Not.

Syst. Herb. Inst. Bot. Zool. Acad. Sci. Uzbekistan 8: 14 .

$\equiv$ Conioselinum pinnatifolium (Korovin) Schischk., 1951, in Schischkin (ed.), Fl. URSS 17: 9.

Type: UZBEKISTAN. "Montes Pamir-Alaj, in valle fl. Tupalang ad locum Kuchtarma, 16 VIII 1937, Lepeschkin \& Kutaleva 323" (lectotype TASH! [TASH002362], designated here; isolectotype TASH! [TASH002363]).

Distribution in MA \& $\mathrm{K}$ : Kazakhstan $(\mathrm{N}$ : Pavlodar prov.; NE: E Kazakhstan prov.; S: S Kazakhstan prov., Zhambyl prov.; SE: Almaty prov.), Kyrgyzstan (Batken prov., Jalal-Abad prov., Ysyk-köl prov., Naryn prov., Osh prov., Talas prov., Chüh prov.), Tajikistan (Kŭhistani Badakhshan, Sughd prov., Khatlon prov., Dushanbe prov.), Uzbekistan (Dzhizak prov., Qashqadarya prov., Surxondarya prov., Toshkent prov.).

Distribution in other Asian countries: Russia, China, India, Pakistan, Mongolia, Afghanistan.

Distribution area: Asia (Arctic, Northern, Central, Eastern, South-Western), Europa.

Conium maculatum L., 1753, Sp. P1.: 243.

$\equiv$ Coriandrum cicuta Crantz, 1767, Stirp. Austr. Fasc. 3: 100. 1767, nom. illeg. (Art. 52.1).

$\equiv$ Cicuta officinalis Crantz, 1767, Cl. umbell. emend.: 98.

$\equiv$ Cicuta major Lam., Fl. franç. (Lamack) 3: 456. 1779, non Mill. (1754)

三 Coriandrum maculatum Roth, 1788, Tent. Fl. Germ. 1: 130.

三Sium conium Vest, 1806, Man. Bot. (Vest): 513.

Selinum conium E. H. L. Krause, 1904, in Deutschl. Fl. (Sturm) ed. 2, 12: 79.

Type: EUROPE. "In Europae cultis, agris, ruderatis, 343.1" (lectotype - LINN, designated by Jafri in Jafri \& El-Gadi 1985: 60) (see also: J.-P. Reduron, 2007: 877).

Distribution in MA \& K: Kazakhstan (W: W Kazakhstan prov.; NE: E Kazakhstan prov.; N: Akmola prov.; S: S Kazakhstan prov., Zhambyl prov.; SE: Almaty prov.), Kyrgyzstan (Batken prov., Jalal-Abad prov., Ysyk-köl prov., Osh prov., Talas prov., Chüh prov.), Tajikistan (Sughd prov., Khatlon prov., Dushanbe prov.), Uzbekistan (Qashqadarya prov., Navoi prov., Dshizak prov. Samarqand prov., Surxondarya prov., Toshkent prov., Namangan prov., Fergona prov.), Turkmenistan (Balkan prov., Akhal prov., Mary prov.). 
Distribution in other Asian countries: Russia, China, Vietnam, India, Pakistan, Afghanistan, Iran, Azerbaidzhan, Georgia, Armenia, Turkey, Iraq, Saudi Arabia, Yemen, Lebanon, Syria, Israel, Jordan, Cyprus.

Distribution area: Asia (Northern, Central, Eastern, South-Western, Mediterranean, W Tropical), Europa, N. Africa, N. America (adv.), S. America (adv.), S. Africa (adv.), Australia \& Oceania (adv.).

Coriandrum sativum L., 1753, Sp. P1.: 256.

三 Coriandrum globosum Salisb., 1796, Prodr. stirp. Chap. Allerton: 166.

三Sium coriandrum Vest, 1806, Man. Bot. [Vest]: 513.

ESelinum coriandrum E. H. L. Krause, 1904, in Deutschl. F1. (Sturm) ed. 2, 12: 163.

Type: EUROPE. "In hortis Musniae, Daniae, \& c., VIII 38" (lectotype UPS-Burser, designated by Jansen 1981: 60); “Leiden, van Royen” (syntype L! [L0053005]).

Distribution in MA \& K: Kazakhstan ( $\mathrm{S}$ : S Kazakhstan prov., Zhambyl prov.; SE:Almaty prov.), Kyrgyzstan (Jalal-Abad prov., Ysyk-köl prov., Osh prov.), Tajikistan (Kŭhistani Badakhshan, Sughd prov., Dushanbe prov.), Uzbekistan (Kara-kalpak, Khorezm prov., Samarqand prov.,), Turkmenistan (Balkan prov., Akhal prov.).

Distribution in other Asian countries: Russia, China, Japonia, Korea, Indonesia, Vietnam, Laos, Myanmar, Bangladesh, Bhutan, Nepal, India, SriLanka, Pakistan, Afghanistan, Iran, Azerbaidzhan, Georgia, Armenia, Turkey, Iraq, Oman, Saudi Arabia, Yemen, Lebanon, Syria, Israel, Jordan, Egypt (Sinai), Cyprus.

Distribution area: Asia (Northern, Central, Eastern, South-Western, Mediterranean, W Tropical \& E Tropical), Europa, Africa, N. America, S. America, Australia \& Oceania. Cultivated and adventive.

Cuminum borszczowii (Regel et Schmalh.) Koso-Pol., 1916, Bull. Soc. Imp. Naturalistes Moscou, n. s. 29: 209.

三Torilis borszczowii Regel et Schmalh., 1878, Trudy Imp. S.-Peterburgsk. Bot. Sada 5(2): 600.

EPsammogeton borszczowii (Regel et Schmalh.) Lipsky, 1904, Trudy Imp. S.-Peterburgsk. Bot. Sada 23: 144 .

Type: KAZAKHSTAN. "Desertum AraloCaspicum, in praeruptii planitiei elevatae UstUrt, 20 IX 1857, Borszczow 459” (lectotypes
LE! [LE00050735, LE00050738], designated by V. M. Vinogradova 2002: 137); isolectotype LE [LE00050737, LE00050739]; UZBEKISTAN. "In deserto inter Taschkent et fluvium Syr-Darja, O. A. Fedtschenko" (syntypes LE [LE00050736, LE00050740]).

Distribution in MA \& K: Kazakhstan (W: Aktobe prov., Mangystau prov.; N: Akmola prov. Central: Karagandy prov., Kzyl-Orda prov.; S: S Kazakhstan prov.), Uzbekistan (Kara-kalpak). Endemic.

Distribution area: Central and South-Western Asia.

Cuminum cyminum L., 1753, Sp. P1.: 254.

ELigusticum cuminum Crantz, 1767, Cl. umbell. emend.: 82.

$\equiv$ Cuminum odorum Salisb., 1796, Prodr. stirp. Chap. Allerton: 165.

$\equiv$ Luerssenia cyminum Kuntze, 1891, Revis. Gen. P1. 1: 268.

ESelinum cuminum E. H. L. Krause, 1904, in Deutschl. F1. (Sturm) ed. 2, 12: 91.

Type: AFRICA. "In Aegypto, Aethiopia, 358.1" (lectotype LINN [LINN-HL358-1], designated by Jansen 1981: 71).

- Cuminum hispanicum auct. non Merat: Bunge, 1852, Beitr. Fl. Russl. (Lehm. Rel. Bot.): 312.

Distribution in MA \& K: Kazakhstan (SE: Almaty prov.), Uzbekistan, Turkmenistan.

Distribution in other Asian countries: Afghanistan, Iran, Azerbaidzhan, Armenia, Iraq, Saudi Arabia.

Distribution area: Asia (Eastern, SouthWestern, W Tropical, E Tropical), Europa, Africa. Cultivated.

Cuminum setifolium (Boiss.) Koso-Pol., 1916, Bull. Soc. Imp. Naturalistes Moscou, n. s. 29: 209.

$\equiv$ Torilis setifolia Boiss., 1856, Diagn. P1. Orient. (ser. 2) 2: 99.

三 Psammogeton setifolius (Boiss.) Boiss., 1872, Fl. Orient. (Boissier) 2: 1079.

$\equiv$ Daucus setifolius (Boiss.) Kuntze, 1887, Trudy Imp. S.-Peterburgsk. Bot. Sada 10: 191, nom. illeg. (Art. 53.1), non Desf. (1798).

$\equiv$ Daucus griffithii M. Hiroe, 1979, Umbelliferae World: 596, nomen.

Type: AFGHANISTAN. "Prope Dair Haj regni Cabulici, 1851, Griffith 548/1024" (holotype G-BOIS! [G00150264]; isolectotypes G-BOIS [G00150324], K! [K000681216, K000681217]).

$=$ Torilis heterotricha Trautv. 1866, Bull. Soc. Imp. Naturalistes Moscou 39(1): 329. 
Type: KAZAKHSTAN. "In deserto sabuloso, fl. Tschu adjacente, VIII 1842, Schrenk 228" (lectotype LE [LE00051030]; designated by V. M. Vinogradova (2002: 137); isolectotypes K!, LE [LE00051029], LE00051031, LE00051032]).

= Cuminum cyminum L. var. setosum Boiss., 1872, Fl. Orient. (Boissier) 2: 1080.

Type: UZBEKISTAN. "In arena fluvii Kisilkum Turkestaniae [in deserto Aralensi], Lehmann" (holotype G-BOIS!).

Distribution in MA \& K: Kazakhstan (Central: Kzyl-Orda prov.; S: S Kazakhstan prov., Zhambyl prov.; SE: Almaty prov.), Kyrgyzstan (Batken prov., Jalal-Abad prov., Osh prov., Chüh prov.), Tajikistan (Sughd prov., Khatlon prov., Dushanbe prov.), Uzbekistan (Kara-kalpak, Khorezm prov., Buxoro prov., Dzhizak prov., Qashqadarya prov., Navoi prov., Namangan prov., Samarqand prov., Surxondarya prov., Sirdarya prov., Toshkent prov., Fergona prov., Andijon prov.), Turkmenistan (Balkan prov., Daşoguz prov., Akhal prov., Lebap prov., Mary prov.).

Distribution in other Asian countries: Pakistan, Afghanistan, Iran, Syria.

Distribution area: Central and South-Western Asia.

Daucus carota L., 1753. Sp. Pl.: 242.

$\equiv$ Caucalis carota Crantz, 1767. Cl. umbell. emend.: 113 .

$\equiv$ Daucus vulgaris Lam., 1779, Fl. franç. (Lamarck) 3: 430).

Type: EUROPA. "In Europae campis exaridis, 340.1" (lectotype LINN! [LINN-HL 340-1], designated by Sainz Lain 1981: 487); cf. Wijnheimer et al. (1988).

= Daucus bactrianus Bunge, 1852, Beitr. Fl. Russl. (Lehm. Rel. Bot.): 136.

Type: UZBEKISTAN. "Am Sarafschan unweit Samarkand, 13 X 1841, Lehmann".

= Daucus exarmatus Korovin, 1948, Not. Syst. Herb. Inst. Bot. Zool. Acad. Sci. Uzbekistan 12: 23.

Type: TAJIKISTAN. "Pamir-alaj, in valle fluv. Kafirnigan meridiem versus urb. Stalinabad [Duchambe], 12 VII 1930, Pazij \& Mironov 1342" (holotype TASH! [TASH002580]).

Distribution in MA \& $\mathbf{K}$ : Kazakhstan (W: Aktobe prov.; NE: E Kazakhstan prov.; Central: Karagandy prov., Kzyl-Orda prov.; S: S Kazakhstan prov., Zhambyl prov.; SE: Almaty prov.), Kyrgyzstan (Batken prov., Jalal-Abad prov., Ysyk-köl prov., Naryn prov., Osh prov., Talas prov., Chüh prov.), Tajikistan (Kŭhistani Badakhshan, Sughd prov., Khatlon prov., Dushanbe prov.), Uzbekistan (Kara- kalpak, Khorezm prov., Buxoro prov., Navoi prov., Dzhizak prov., Samarqand prov., Qashqadarya prov., Surxondarya prov., Sirdarya prov., Toshkent prov., Namangan prov., Fergona prov., Andijon prov.), Turkmenistan (Balkan prov., Akhal prov., Lebap prov., Mary prov.).

Distribution in other Asian countries: Russia, China, Japan, Nepal, India, Sri lanka, Pakistan, Afghanistan, Iran, Azerbaidzhan, Georgia, Armenia, Turkey, Iraq, Oman, UAE, Saudi Arabia, Yemen, Lebanon, Syria, Israel, Jordan, Cyprus.

Distribution area: Asia (Northern, Central, Eastern, South-Western, Mediterranean), Europa, Africa, N. America (adv.), Australia \& Oceania (adv.).

Dichoropetalum golestanicum (Rech. f.) Pimenov et Kljuykov, 2007, Willdenowia 37(2): 485.

$\equiv$ Johrenia golestanica Rech.f, 1987, in Fl. Iranica (Rechinger), 162: 376, tab. 304.

Type: IRAN. "Persia, Gorgan: Jangal-e Golestan prope Tang-e Rah, 420-500 m, 13-14 X 1976, Termé \& Matiné 35109-E" (lectotype W! [W 19800011846]; designated here by Pimenov; isolectotype ibid. 13 X 1976, Termé \& Matiné 35110-E, W [W 1980-0011849]).

Distribution in MA \& K: Turkmenistan (Balkan prov.).

Distribution in other Asian countries: Iran.

Distribution area: South-Western Asia.

Dichoropetalum platycarpum (Boiss.) Pimenov et Kljuykov, 2007, Willdenowia 37(2): 487.

$\equiv$ Johrenia platycarpa Boiss., 1844, Ann. Sci. Nat. sér. 3, 1: 308.

Type: IRAN. "In monte Elbourz, Aucher-Eloy 4582" (lectotype G-BOIS!; designated by Pimenov et Jacquemoud (2020: 64); isolectotypes FI!, G! [G00359590], K [K000681161], P! [P00753021, P00753022, P00753023]).

Distribution in MA \& K: Turkmenistan (Balkan prov.).

Distribution in other Asian countries: Iran.

Distribution area: South-Western Asia.

Dimorphosciadium gayoides (Regel et Schmalh.) Pimenov, 1975, Byull. Moskovsk. Obshch. Isp. Prir. Otd. Biol. 80(3): 83.

$\equiv$ Meum gayoides Regel et Schmalh., 1882, Izv. Obshch. Ljubit. Estestv. 34(2) (Descr. Pl. Nov. Rar. Fedtsch.): 32. 
$\equiv$ Ligusticum gayoides (Regel et Schmalh.) Korovin, 1925, Bull. Inst. Pedol. Geobot. Univ. As. Centr. 1: 108, in nota.

$\equiv$ Pachypleurum gayoides (Regel et Schmalh.) Schischk, 1950, in Schischkin (ed.), Fl. URSS 16: 582, p. p.

Type: KYRGYZSTAN. "Kokand, traj. KtschiAlai, 26 VI 1871, O. A. Fedtschenko" (holotype LE! [LE01066098]).

Distribution in MA \& K: Kazakhstan (S: S Kazakhstan prov.), Kyrgyzstan (Naryn prov., Osh prov.), Tajikistan (Kŭhistani Badakhshan, Dushanbe prov.), Uzbekistan (Toshkent prov.).

Distribution in other Asian countries: Afghanistan.

Distribution area: Central and South-Western Asia.

Dorema aitchisonii Korovin ex Pimenov, 1983, in Vvedensky (ed.), Opred. Rast. Sred. Azii 7: 377.

EFerula michaelii M. Panahi et al. 2015, Taxon 64(4): 780.

Type: TURKMENISTAN. "Desertum Karakum austro-orientalis, in denudationibus arenosis depressionis lacus Er-oilan-duz. 15 V 1925. Korovin $341,342,343,344,345$ " (holotype TASH! [TASH002527-TASH002537]).

Distribution in MA \& K: Tajikistan (Khatlon prov., Dushanbe prov.), Turkmenistan (Balkan prov., Akhal prov., Mary prov.).

Distribution in other Asian countries: Afghanistan, Iran.

Distribution area: South-Western Asia.

Dorema badhysi Pimenov, 1983, in Vvedensky (ed.), Opred. Rast. Sred. Azii 7: 379, 314.

Type: AFGHANISTAN. "Afghanistan, Badghys. 14-16 V 1885. Aitchison 462" (holotype LE!; isotype K! [K000685784]).

- Dorema glabrum auct. non Fisch. et C. A. Mey.: Aitch. 1888, Trans. Linn. Soc. Bot. London (2 ser.) 3: 70 .

- Dorema gummiferum (Jaub. et Spach) Korovin, 1950, in Nikitin (ed.), Fl. Turkmen. 5: 247, nom. illeg. (Art. 41.5), sine basion.

- Dorema gummiferum (Jaub. et Spach) K. Korol., 1951, in Schischkin (ed.), Fl. URSS 17: 162, non Diserneston gummiferum Jaub. et Spach.

- Dorema hyrcanum auct. non Koso-Pol.: Rech. f. et Riedl, 1963, in Biol. Skr. 13, 4 (Symb. Afghan. 5): 50 .

Distribution in MA \& K: Turkmenistan (Akhal prov., Mary prov.).
Distribution in other Asian countries: Afghanistan.

Distribution area: South-Western Asia.

Dorema balchanorum Pimenov, 1983, in Vvedensky (ed.), Opred. Rast. Sred. Azii 7: 378, 314.

Type: TURKMENISTAN. "Turcomania, Montes Balchan Major, declivum septentrionale, in ascendi ad montium Orlan, Pat'ma. 10 V 1975. Pimenov 351" (holotype MW! [MW0594061, MW9594062, MW594063]).

Distribution in MA \& K: Turkmenistan (Balkan prov.). Endemic.

Distribution area: South-Western Asia.

Dorema hyrcanum Koso-Pol., 1921, Bot. Mater. Gerb. Glavn. Bot Sada R.S.F.S.R. 2(1): 67, quoad typus.

$\equiv$ Ferula hyrcana (Koso-Pol.) Puchałka, Spalik, Panahi et Piwczynski, 2015, Taxon 64(4): 780.

Type: TURKMENISTAN. "In prov. Transcaspica, Ssjunt. 14 V 1916. B. A. Fedtschenko 169" (lectotype LE!, designated by Pimenov 1988: $87)$.

Distribution in MA \& K: Turkmenistan (Balkan prov., Akhal prov.).

Distribution in other Asian countries: Iran.

Distribution area: South-Western Asia.

Dorema karataviense Korovin, 1962, Trudy Inst. Bot. Akad. Nauk Kazakhst. 13: 261.

Type: KAZAKHSTAN. "Montes Karatau Syrdariensis, extremitas septentrionalis, Uzunbulak Dzhalgyz-agach, 08 VI 1929, Korovin 329, 330" (holotype TASH! [TASH002538, TASH002539]).

Distribution in MA \& K: Kazakhstan (Central: Kzyl-Orda prov.). Endemic.

Distribution area: Central Asia.

Note: Closely related to $D$. sabulosum.

Dorema kopetdaghense Pimenov, 1983, in Vvedensky (ed.), Opred. Rast. Sred. Azii 7: 378.

Type: TURKMENISTAN. Montes Kopet-Dagh, angustis Firyuza. 21 V 1972. Pimenov 151 (holotype MW! [MW0594071, MW0594072]; isotype LE!).

- Dorema hyrcanum auct. non Koso-Pol.: Rech. f. 1987, in Fl. Iranica (Rechinger) 162: 384, p. p.

Distribution in MA \& K: Turkmenistan (Akhal (prov.).

Distribution in other Asian countries: Iran.

Distribution area: South-Western Asia. 
Dorema microcarpum Korovin 1947, Not. Syst. Herb. Inst. Bot. Zool. Acad. Sci. Uzbekistan 8: 6.

Type: KYRGYZSTAN. "Tian-Schan, mont. Ferganici, vallis fl. Kara-su [Tian-Schan centralis, distr. Ketmen-Tube, systema fl. Kara-su, 15-20 km infra ostium Ujunkur]. 14 V 1927. Korovin 612, 613, 614" (holotype TASH! [TASH002540-TASH002542]).

Distribution in MA \& K: Kyrgyzstan (JalalAbad prov., Naryn prov., Osh prov.), Uzbekistan (Namangan prov., Fergona prov.). Endemic.

Distribution area: South-Western Asia.

Dorema sabulosum Litv. 1913, Sched. Herb. F1 Ross. 8(2): N 2404a-c.

Type: TURKMENISTAN. "Regio Transcaspica, distr. Merw, in arenosis prope stationem viae ferreae Karaul-Kuju. 06 V 1911. Androssov 2404a-c" (lectotype LE!; designated by V. M. Vinogradova 1999: 86; isolectotypes BP!, C! [C10008395C10008397], GH [GH00076218]?], H [H1397796], M [M0173174], MHA!, MW! [MW0594072MW0594075], NSK [NSK0000425-NSK0000427], S! [S-G-2132]).

- Dorema ammoniacum auct. non D. Don: Bunge, 1852, Beitr. Fl. Russl. (Lehm. Rel. Bot.): 132.

Distribution in MA \& K: Kazakhstan (Central: Kzyl-Orda prov.; S: S Kazakhstan prov.), Tajikistan (Khatlon prov.), Uzbekistan (Kara-kalpak, Buxoro prov., Navoi prov., Surxondarya prov.), Turkmenistan (Balkan prov., Daşoguz prov., Akhal prov., Lebap prov., Mary prov.). Endemic.

Distribution area: Central and South-Western Asia.

Echinophora sibthorpiana Guss., 1832, Fl. Sic. Prodr. Suppl. 1: 69.

$\equiv$ Echinophora tenuifolia L. var. sibthorpiana (Guss.) Griseb., 1843, Spic. Fl. Rumel. 1: 386.

$\equiv$ Chrysosciadium sibthorpianum (Guss.)

Tamamsch., 1967, in Fl. Kavkaza (Grossheim) ed. 2 7: 24, tab. 2, fig. 3, map 15, nom. inval. (Art. 35.1).

$\equiv$ Echinophora tenuifolia $\mathrm{L}$. subsp. sibthorpiana (Guss.) Tutin, 1967, Feddes Repert. 74(1-2): 31.

$\equiv$ Anisosciadium tenuifolium (L.) Tamamsch. subsp. sibthorpianum (Guss.) Tamamsch., 1975, Abstr. XII Int. Bot. Congr. 1: 107.

Type: TURKEY. "From Mount Olympus [Bursa], Sibthorp" (lectotype OXF!: designated by Pimenov, 2016, in Bot. Zhurn. 101(10): 1227).

Distribution in MA \& K: Kazakhstan ( $\mathrm{S}$ : S Kazakhstan prov.), Kyrgyzstan (Batken prov., Osh prov.), Tajikistan (Sughd prov.), Uzbekistan (Buxoro prov., Dzhizak prov., Samarqand prov., Qashqadarya prov., Surxondarya prov., Sirdarya prov., Toshkent prov., Namangan prov.) Turkmenistan (Akhal prov., Lebap prov.).

Distribution in other Asian countries: Russia, Afghanistan, Iran, Azerbaidzhan, Georgia, Armenia, Turkey, Lebanon, Syria, Cyprus.

Distribution area: Asia (N, SW, Mediterranean), Europa (SE).

Elaeosticta alaica (Lipsky) Kljuykov, Pimenov et V. N. Tikhom., 1976, Byull. Moskovsk. Obshch. Isp. Prir. Otd. Biol. 81(6): 92.

$\equiv$ Carum alaicum Lipsky, 1904, Trudy Imp. S.-Peterburgsk. Bot. Sada 23: 127.

三Bunium alaicum (Lipsky) B. Fedtsch., 1915, Rastit. Turkest.: 612.

EScaligeria alaica (Lipsky) Korovin, 1928, Byull. Sredne-Asiatsk. Univ. (ser. 8b, Bot.) 2: 39.

Type: KYRGYZSTAN. "Prov. Fergana, prope st. Langar ad fl. Taldyk in decliv. orientalis, 55006000', 26 VI 1901, Alexeenko 834" (lectotype LE! [LE00051471], designated by Kljuykov 1983: 144); "Inter Mady et Langar, 26 VI 1901, B. A. Fedtschenko" (syntype LE! [LE00051470]).

$=$ Scaligeria alaica (Lipsky) Korovin var. multiradiata Korovin, 1928, Byull. Sredne-Asiatsk. Univ. (ser. 8b, Bot.) 2: 39.

Type: TAJIKISTAN. "Karategin, in valle Sorbuch, 8000', 17 VIII 1881, A. Regel" (holotype LE! [LE00051211]).

Distribution in MA \& K: Kyrgyzstan (Batken prov., Jalal-AbadChüh prov.), Uzbekistan (Toshkent prov.). Endemic.

Distribution area: South-Western Asia.

Elaeosticta allioides (Regel et Schmalh.) Kljuykov, Pimenov et V. N. Tikhom., 1976, Byull. Moskovsk. Obshch. Isp. Prir. Otd. Biol. 81(6): 92.

$\equiv$ Conopodium allioides Regel et Schmalh., 1878, Trudy Imp. S.-Peterburgsk. Bot. Sada 5(2): 588.

$\equiv$ Carum allioides (Regel et Schmalh.) Franch., 1883, Ann. Sci. Nat. sér. 6, 16: 293.

EScaligeria allioides (Regel et Schmalh.) Boiss., 1888, Fl. Orient. (Boissier) Suppl.: 255.

Type: UZBEKISTAN. 'Kokschar, 6-7000', Tschotkal (Taschkentes Alatau), VIII 1876, A. Regel" (lectotype LE! [LE00051626], designated by Kljuykov 1983: 153); isolectotype G-BOIS!); Tschotkal. 08.1876. A. Regel (syntypes LE! [LE00051628, LE00051629].

Distribution in MA \& K: Kazakhstan (S: S Kazakhstan prov., Zhambyl prov.), Kyrgyzstan (Batken prov., Jalal- Abad prov., Osh prov., 
Talas prov., Chüh prov.), Tajikistan (Kŭhistani Badakhshan, Sughd prov., Khatlon prov., Dushanbe prov.), Abad prov., Osh prov., Talas prov., Tajikistan (Kŭhistani Badakhshan, Sughd prov., Khatlon prov., Dushanbe prov.), Uzbekistan (Buxoro prov., Navoi prov., Dzhizak prov., Samarqand prov., Qashqadarya prov., Surxondarya prov., Sirdarya prov., Toshkent prov.), Turkmenistan (Balkan prov., Akhal prov., Lebap prov.).

Distribution in other Asian countries: Afghanistan, Iran.

Distribution area: South-Western Asia.

Elaeosticta bucharica (Korovin) Kljuykov, Pimenov et V. N. Tikhom., 1976, Byull. Moskovsk. Obshch. Isp. Prir. Otd. Biol. 81(6): 92.

$\equiv$ Scaligeria bucharica Korovin, 1924, Bot. Mater. Gerb. Glavn. Bot. Sada R.S.F.S.R. 5(6): 79.

Type: UZBEKISTAN. "Buchara, Baba-tag, prope pag. Kel'-Bulak. 29 VI 1914, Popov 383" (lectotype TASH! [TASH002189]; designated as "typus" by Kljuykov 1983: 144; isolectotype LE! [LE00051213]).

Distribution in MA \& K: Tajikistan (Kŭhistani Badakhshan, Khatlon prov., Dushanbe prov.), Uzbekistan (Qashqadarya prov., Surxondarya prov.). Endemic.

Distribution area: South-Western Asia.

Elaeosticta conica Korovin, 1948, Not. Syst. Herb. Inst. Bot. Zool. Acad. Sci. Uzbekistan 12: 31.

$\equiv$ Scaligeria conica (Korovin) Korovin, 1950, in Schischkin (ed.), Fl. URSS 16: 209.

Type: UZBEKISTAN. "Pamiro-Alaj occidentalis in regione Guzar ad locus Dultaly-Kyzyl [In montibus humilioribus gypsaceis SE oppidum Gusar, locum Dultaly-Kysyl prope pag. Kysylchi, in detrito argilloso rubro, declivum septentionale]. 19 V 1935. Slovinsky K-7"' (lectotype TASH! [TASH002186]; designated here; isotypes LE! [LE00051253], TASH [TASH002187], ibid., mont. Nagora-chona, ad gypsaceas calcareas. $10 \mathrm{~V}$ 1935. T. Pazij (paratype TASH).]).

Distribution in MA \& K: Tajikistan (Khatlon prov.), Uzbekistan (Qashqadarya prov., Surxondarya prov.), Turkmenistan (Lebap prov.). Endemic.

Distribution area: South-Western Asia.

Elaeosticta ferganensis (Lipsky) Kljuykov, Pimenov et V. N. Tikhom., 1976, Byull. Moskovsk. Obshch. Isp. Prir. Otd. Biol. 81(6): 92.

$\equiv$ Scaligeria ferganensis Lipsky, 1904, Trudy Imp. S.-Peterburgsk. Bot. Sada 23: 136. $\equiv$ Conopodium ferganense (Lipsky) Druce, 1917, Rep. Bot. Exch. Cl. Brit. Isles 4 (Suppl. 2): 616.

Type: KYRGYZSTAN. Turkestan, Andizhan distr., Arslanbob. 01-02 VI 1899, Litvinov (lectotype LE! [LE00051214], designated by Kljuykov 1983: 152); Yassy. 30 V 1880. A. Regel (syntypes LE! [LE00051215-LE00051218]).

= Carum korshinskii Lipsky, 1904, Trudy Imp. S.-Peterburgsk. Bot. Sada 23: 128.

= Bunium korshinskii (Lipsky) B. Fedtsch., 1915, Rastit. Turkest.: 612.

= Scaligeria ferganensis Lipsky var. korshinskii (Lipsky) Korovin, 1928, Trudy Sredne-Asiatsk. Gosud. Univ. (ser. 8b, Bot.) 2: 56.

= Scaligeria korshinskii (Lipsky) Korovin, 1950, in Schischkin (ed.), Fl. URSS 16: 216, p. p.

Type: KYRGYZSTAN. "Montes Alai, locus dictus Olgin Lug (pratum Olgae), in juniperetum in declivi boreali. 15 VII 1895. Korshinsky 3182" (lectotype LE! [LE00051511], designated as holotypus by V. M. Vinogradova 1997a: 98; isolectotypes LE [LE00051510, LE00051512].

Distribution in MA \& K: Kyrgyzstan (Batken prov., Jalal-Abad prov., Naryn prov., Osh prov., Talas prov., Chüh prov.), Tajikistan (Kŭhistani Badakhshan), Uzbekistan (Toshkent prov.). Endemic.

Distribution area: South-Western Asia.

Elaeosticta hirtula (Regel et Schmalh.) Kljuykov, Pimenov et V. N. Tikhom., 1976, Byull. Moskovsk. Obshch. Isp. Prir. Otd. Biol. 81(6): 92.

$\equiv$ Conopodium hirtulum Regel et Schmalh., 1882, Izv. Obshch. Ljubit. Estestv. 34(2) (Descr. Pl. Nov. Rar. Fedtsch.): 28.

$\equiv$ Scaligeria hirtula (Regel et Schmalh.) Lipsky, 1904, Trudy Imp. S.-Peterburgsk. Bot. Sada 23: 134.

Type: KYRGYZSTAN. "In montibus Maili, Krause" (lectotype LE! [LE00051631], designated by Kljuykov 1983: 152; isolectotypes LE! [LE00051632, LE00051633]).

= Scaligeria polyantha Rech. f. 1963, in Biol. Skr. 13(4) (Symb. Afghan. 5): 121, fig. 84, 85.

Type: AFGHANISTAN. "NE Afghanistan, Badakhshan: Faizabad, 1100-1900 m, 11 VII 1948, Edelberg 1367" (lectotype W! [W0054258]; designated here by Pimenov; isolectotype C).

= Scaligeria afghanica Podlech, 1970, Mitt. Bot. Staatsamml. München 8: 177.

Type: AFGHANISTAN. "Nordost-Afghanistan, Prov. Takhar: Unteres Namabab-Tal, Losshanse westlich von Taqcha Khana, 1500 m, 24 VI 1965, Podlech 11497" (holotype M! [M01729523]; isotype MSB [MSB003201]). 
= Scaligeria oedibasioides Kamelin, 1977, Novit. Syst. P1. Vasc. 14: 187.

Type: UZBEKISTAN. "Usbekistania australis, regio collino-montana versus meridiem ad viam Dechkanabad-Derbent, montes Kuruk-dagana, ad declivium meridionalem trajecto Tally, ad argillas salsas. 19 V 1967, Botschantzev 121" (holotype LE! [LE00051237).

Distribution in MA \& K: Kyrgyzstan (Batken prov., Jalal-Abad prov., Osh prov.), Tajikistan (Kŭhistani Badakhshan, Sughd prov., Khatlon prov., Dushanbe prov.), Uzbekistan (Buxoro prov., Dzhizak prov., Samarqand prov., Qashqadarya prov., Surxondarya prov., Toshkent prov.), Turkmenistan (Lebap prov.).

Distribution in other Asian countries: Afghanistan.

Distribution area: South-Western Asia.

Elaeosticta knorringiana (Korovin) Korovin, 1948, Not. Syst. Herb. Inst. Bot. Zool. Acad. Sci. Uzbekistan 12: 32.

EScaligeria knorringiana Korovin, 1928, Trudy Sredne-Asiatsk. Gosud. Univ. (ser. 8b, Bot.) 2: 34.

Type: KYRGYZSTAN. "Fergana orientalis, distr. Dzhelalabad, in collibus sterilibus inter Bazar-kurgan et Dzhelalabad, 29 VI1927, Korovin 402, 405" (lectotype TASH! [TASH002191], designated by Kljuykov 1983: 150); isolectotypes LE! [LE00051221], MHA!, TASH (404, 410, 416, 417, 418) [TASH002192 - TASH002196]; Fergona prov., meteostation Schatak-tepe in $5 \mathrm{rm}$ from kishlak Zorkent ). 02.06/1912. Knorring 54 (syntypes LE [LE00051228, LE00051229, LE00051230, LE00051231, LE00051232]; Angustiis Ak-Bura 24 VI 1878. Kushakevich (syntype LE [LE00051222, LE00051223, LE00051224, LE00051226], inter Andizhan et Karasu. 25 VIII 1880). A. Regel (syntype LE [LE00051225]; vallis Alaicus, 12000'. 08 VII 1891. Brzhetskiy (syntype LE [LE00051227].

= Hyalolaena collina Korovin, 1948, Not. Syst. Herb. Inst. Bot. Zool. Acad. Sci. Uzbekistan 12: 22.

Type: UZBEKISTAN. "Vallis Ferganica, in collibus argillosis pr. st. V. F. Chakulabad, solo subsalso. 25 V 1932. Zakrzewski 1033" (holotype TASH! [TASH002349]; isotype TASH (1032) [TASH002350]).

Distribution in MA \& K: Kyrgyzstan (Batken prov., Jalal-Abad prov., Osh prov.), Uzbekistan (Namangan prov.). Endemic.

Distribution area: South-Western Asia.
Elaeosticta korovinii (Bobr. ex Korovin) Kljuykov, Pimenov et V. N. Tikhom., 1976, Byull. Moskovsk. Obshch. Isp. Prir. Otd. Biol. 81(6): 93.

EScaligeria korovinii Bobr. ex Korovin, 1950, in Schischkin (ed.), Fl. URSS 16: 593, 211.

Type: TURKMENISTAN. "Montes Balchany Majores, declivia australia in schistosis, ca. 600 m s. m. 04 VI 1928. Bobrov \& Jarmolenko 312" (holotype LE! [LE00051233]).

Distribution in MA \& K: Turkmenistan (Balkan prov.). Endemic.

Distribution area: South-Western Asia.

Elaeosticta lutea (M. Bieb. ex Hoffm.) Kljuykov, Pimenov et V. N. Tikhom., 1978, Byull. Moskovsk. Obshch. Isp. Prir. Otd. Biol. 83(6): 105.

$\equiv$ Bunium luteum M. Bieb. ex Hoffm., 1814, Gen. P1. Umbell.: 108.

$\equiv$ Sium luteum (M. Bieb. ex Hoffm.) Spreng. 1818, Sp. Umbell.: 92.

三Drepanophyllum luteum (M. Bieb. ex Hoffm.) Eichw. 1830, Skizze: 257.

$\equiv$ Muretia lutea (M. Bieb. ex Hoffm.) Boiss., 1872, Fl. Orient. (Boissier) 2: 858.

$\equiv$ Muretia tanaicensis Boiss., 1844, Ann. Sci. Nat. sér. 3, 1: 143. 1844.

Type: EUROPE, RUSSIA. "In agro Sareptano" (holotype LE-Bieb!, designated by V. M. Vinogradova in LE; Kljuykov, 1983: 146).

Distribution in MA \& K: Kazakhstan (W: W Kazakhstan prov., Aktobe prov., Mangystau prov.; Central: Karagandy prov.).

Distribution in other Asian countries: Russia.

Distribution area: Asia (N, Central), Europa.

Elaeosticta paniculata (Korovin) Kljuykov et Pimenov, 1981, in S. K. Cherepanov, Sosud. Rast. SSSR: 19.

$\equiv$ Hyalolaena paniculata Korovin, 1948, Not. Syst. Herb. Inst. Bot. Zool. Acad. Sci. Uzbekistan 12: 20.

Type: UZBEKISTAN. Jugum Zeravschanicum in valle fl. Langar, in Agropyretis ["Systema fl. Kaschka-darja, pars occidentalis jugi Seravschanici, declivum australe, prope pag. Langar, 04 VII 1938, Kudrjaschov \& Sumnevich 753" (holotype TASH! [TASH002352]; isotypes TASH [TASH002353, TASH002354]).

$=$ Elaeosticta seravschanica Kljuykov et Pimenov 1979, Bot. Zhurn. (Leningrad) 64(9): 1312. 
Type: UZBEKISTAN. "Uzbekistania, jugum Zeravschanicum, inter Tersak et Aman-kutan. 23 V 1971. Pimenov \& al. 347" (holotype LE! [LE00051254]).

Distribution in MA \& K: Uzbekistan (Buxoro prov., Samarqand prov., Qashqadarya prov., Surxondarya prov.), Tajikistan (Sughd prov.). Endemic.

Distribution area: South-Western Asia.

Elaeosticta platyphylla (Korovin) Kljuykov, Pimenov et V. N. Tikhom., 1976, Byull. Moskovsk. Obshch. Isp. Prir. Otd. Biol. 81(6): 93.

$\equiv$ Scaligeria platyphylla Korovin, 1950, in Schischkin (ed.), Fl. URSS 16: 592, 211.

Type: TURKMENISTAN. Turkmen SSR, ad declivibus Pistaceetis prope Kuschka, 01 VII 1931, Androssov (holotype ASH!; isotype LE! [LE00051238]).

Distribution in MA \& K: Turkmenistan (Akhal prov., Mary prov.). Endemic.

Distribution area: Central and South-Western Asia.

Elaeosticta polycarpa (Korovin) Kljuykov, Pimenov et V. N. Tikhom., 1978, Byull. Moskovsk. Obshch. Isp. Prir. Otd. Biol. 83(6): 106.

$\equiv$ Scaligeria polycarpa Korovin, 1924, Bot. Mater. Gerb. R.S.F.S.R. 5(6): 80.

Type: UZBEKISTAN. "Samarkand prov., near vill. Kurkat, serpentine slopes, 22 VI 1923, Popov \& Vvedensky 197" (lectotype LE! [LE00051239], designated here; isolectotypes LE [LE00051240], TASH $(196,198,199,200,202)$ [TASH002197 TASH002201]).

= Scaligeria takharensis Podlech, 1970, Mitt. Bot. Staatsamml. München 8: 179, fig. 10, 11.

Type: AFGHANISTAN. "Nordost-Afghanistan, Prov. Takhar: Badam-Darrah sudlich von Taluqua, Losshange sudlich des Ortes, 1000 m, 19 VI 1965, Podlech 11431" (holotype M! [M0172949]; isotype MSB [MSB003202]).

Distribution in MA \& K: Kyrgyzstan (Batken prov.), Tajikistan (Kŭhistani Badakhshan, Sughd prov., Khatlon prov., Dushanbe prov.), Uzbekistan (Dzhizak prov., Samarqand prov., Qashqadarya prov., Surxondarya prov., Toshkent prov.), Turkmenistan (Lebap prov., Mary prov.).

Distribution in other Asian countries: Afghanistan.

Distribution area: South-Western Asia.
Elaeosticta samarkandica (Korovin) Kljuykov, Pimenov et V. N. Tikhom., 1976, Byull. Moskovsk. Obšč. Isp. Prir. Otd. Biol. 81(6): 93.

EScaligeria samarkandica Korovin, 1928, Trudy Sredne-Asiatsk. Gosud. Univ. (ser. 8b, Bot.) 2: 44.

Type: TAJIKISTAN "Prov. Samarkand, distr. Chodzhent, Kul-keryz. 13 V 1924. Popov \& Vvedensky 643" (lectotype TASH! [TASH002202]; designated as "typus" by Kjuykov (1983: 150); isolectotypes LE! [LE00051241], TASH (615) [TASH002205]).

Distribution in MA \& K: Kyrgyzstan (Batken prov.), Tajikistan (Sughd prov.). Endemic.

Distribution area: South-Western Asia.

Elaeosticta transcaspica (Korovin) Kljuykov, Pimenov et V. N. Tikhom., 1976, Byull. Moskovsk. Obshch. Isp. Prir. Otd. Biol. 81(6): 93.

EScaligeria transcaspica Korovin, 1928, Trudy Sredne-Asiatsk. Gosud. Univ. (ser. 8b, Bot.) 2: 60, tab. 1, fig. 14.

Type: TURKMENISTAN. "SW Kara-kum desertum, terrassa superior fluminis Kuschka, prope stationem Kalai-Mor. 13 V 1925. Korovin 331" (lectotype TASH! [TASH002207],], designated by Kljuykov 1983: 152; sett. Kuschkinsky. $10 \mathrm{~V}$ 1895. Korshinsky (syntypes LE [LE00051242LE00051244]).

Distribution in MA \& K: Turkmenistan (Mary prov.).

Distribution in other Asian countries: Afghanistan.

Distribution area: South-Western Asia.

Elaeosticta transitoria (Korovin) Kljuykov, Pimenov et V. N. Tikhom., 1978, Byull. Moskovsk. Obshch. Isp. Prir. Otd. Biol. 83(6): 105.

$\equiv$ Muretia transitoria Korovin, 1924, Bot. Mater. Gerb. Glavn. Bot. Sada R.S.F.S.R. 5(6): 85.

$\equiv$ Bunium transitorium (Korovin) M. Hiroe, 1979, Umbelliferae World: 683.

Type: KYRGYZSTAN. "Regio Heptapotamica, Pishpek prov., Usungur distr., northern slope of Alexandr Range, fl. Alamedin valley, Mt. Shekule. 06 VII 1916. Kushnirenko 623" (lectotype LE! [LE00051664], designated by V. M. Vinogradova (2002:139); isolectotypes LE! [LE00051665], TASH! [TASH002312, TASH002314]); "Regio Heptapotamica, Pishpek distr. Prope riv. Alamedin. 27 VI 1916. Sovetkina \& Chausova 1804" (syntype LE! [LE00051666]). 
= Elaeosticta kuramensis Korovin, 1948, Not. Syst. Herb. Inst. Bot. Zool. Acad. Sci. Uzbekistan 12: 31 .

= Scaligeria kuramensis (Korovin) Korovin, 1950, in Schischkin (ed.), Fl. URSS 16: 211, in obs.

Type: UZBEKISTAN. "Montes Tian-schan occidentalis, in valle fl. Angren, in promontoriis prope pag. Kul-ata. $13 \mathrm{~V}$ 1928. Granitov \& Mironov 226" (holotype TASH! [TASH002188]).

Distribution in MA \& K Asia: Kazakhstan (S: S Kazakhstan prov., Zhambyl prov.), Kyrgyzstan (Jalal-Abad prov., Talas prov.), Tajikistan (Sughd prov.), Uzbekistan (Toshkent prov.). Endemic.

Distribution area: South-Western Asia.

Elaeosticta tschimganica (Korovin) Kljuykov, Pimenov et V. N. Tikhom., 1976, Byull. Moskovsk. Obshch. Isp. Prir. Otd. Biol. 81(6): 93.

$\equiv$ Scaligeria tschimganica Korovin, 1928, Trudy Sredne-Asiatsk. Gosud. Univ. (ser. 8b, Bot.) 2: 62.

Type: UZBEKISTAN. "[Tian-Schan occidentalis, Taschkent Ala-tau] Vallis Chimganensis, 30 VI 1925, Korovin" (lectotype TASH! [TASH002215]; designated by Kljuykov (1983: 153); isolectotypes LE! [LE00051246]; "Charvak. 09 VII 1926. Korovin" (syntypes LE [LE00051246, LE00051247]).

Distribution in MA \& K: Kyrgyzstan (JalalAbad prov., Osh prov.), Tajikistan (Sughd prov.), Uzbekistan (Toshkent prov.). Endemic.

Distribution area: South-Western Asia.

Elaeosticta ugamica (Korovin) Korovin, 1948, Syst. Herb. Inst. Bot. Zool. Acad. Sci. Uzbekistan 12: 31 , in obs.

$\equiv$ Scaligeria ugamica Korovin, 1924, Bot. Mater. Gerb. Glavn. Bot. Sada R.S.F.S.R. 5(6): 78.

Type: UZBEKISTAN. "Syr-Darya prov., Taschkent distr., inn valle fl. Ugam, Ming-bulak. 16 VIII 1922 riv. Boguchalpak, 12 VII 1922, Simonova \& Batueva" (lectotype TASH! [TASH002219]; designated as "typus" by Kljuykov (1983: 146); isolectotypes LE! [LE00051249], TASH! [TASH002218]).

Distribution in MA \& K: Kazakhstan (S: S Kazakhstan prov.), Kyrgyzstan (Jalal-Abad prov., Osh, Talas prov.), Uzbekistan (Toshkent prov.). Endemic.

Distribution area: South-Western Asia.

Elaeosticta vvedenskyi (Kamelin) Kljuykov, Pimenov et V. N. Tikhom., 1978, Byull. Moskovsk. Obshch. Isp. Prir. Otd. Biol. 83(6): 106. $\equiv$ Scaligeria vvedenskyi Kamelin, 1973, Bot. Zhurn. (Leningrad) 58(5): 630.

Type: UZBEKISTAN. "Uzbekistania, ad declivitates australes montium Koitasch prope pagum Koitasch, in locis lapidosis, 28 VI 1971, Botschantzev \& Kamelin 538" (holotype LE! [LE00051250]; isotypes LE! [LE00051251, LE00051251]).

Distribution in MA \& K: Kazakhstan (S: S Kazakhstan prov.), Uzbekistan (Kara-kalpak, Buxoro prov., Dzhizak prov., Navoi prov.). Endemic.

Distribution area: South-Western Asia.

Elwendia afghanica (Beauverd) Pimenov et Kljuykov, 2013, Pl. Syst. Evol. 299 (5): 1002.

इBunium afghanicum Beauverd, 1929, Bull. Soc. Bot. Genève (sér. 2) 21: 227.

Type: AFGHANISTAN. Affghania, Hari-Rud valley, in agris cultis, 25-26 IV1885, Aitchison 312" (lectotype G! [G00367675]; designated here; isolectotypes K! [K000685710], LE!).

= Bunium cylindricum (Boiss. et Hohen.) Freyn subsp. badghysi Korovin, 1927, Byull. Sredne-Asiatsk. Gosud. Univ. 15: 127.

= Bunium badghysi (Korovin) Korovin, 1950, in Schischkin (ed.), Fl. URSS 16: 410.

Type: TURKMENISTAN. "In collibus Badghyz: prope Berdy-klytsch, 11 IV 1916, Korovin 257" (holotype TASH! [TASH002281]; isotype], designated here by Pimenov; isolectotype TASH! [TASH002280]).

= Bunium caroides (Boiss.) Hausskn. ex Bornm. f. elegans Parsa, 1966, Fl. Iran. 9: 177, fig. 60.

Type: IRAN. "E: Khorassan, Torbat-i-Haydari, V-VI 1957, Parsa" (holotype TEH).

Distribuion in MA \& K: Turkmenistan (Mary prov.).

Distribution in other Asian countries: Afghanistan, Iran.

Distribution area: South-Western Asia.

Elwendia angreni (Korovin) Pimenov et Kljuykov, 2013, Pl. Syst. Evol., 299 (5): 1002.

$\equiv$ Bunium angreni Korovin, 1927, Byull. SredneAsiatsk. Gosud. Univ. 15: 123.

Type: "UZBEKISTAN. Tian-Schan occidentalis, in valle fl. Angren, in regione alpina prope Kamtschik-say, 09 VIII 1924, Korovin E605" (lectotype TASH! [TASH002277], designated by Korovin (1950: 404); isolectotype TASH [TASH002275, TASH002276]; "Ripa sinistra fl. Angren, in cursu medio fluminis Ak-sai (N Ablyk), declivia mol- 
lia, 20 VI 1924, Korovin E317" (syntypes TASH [TASH002278, TASH002279].

Distribution in MA \& K: Uzbekistan (Toshkent prov) Endemic.

Note: In the protologue, Korovin (1927) did not indicate definitely in which herbarium the type (lectotype) of Bunium angreni was stored. He make this in "Flora of the URSS" (Korovin, 1950: 404).

Distribution area: South-Western Asia.

Elwendia badachschanica (Kamelin) Pimenov et Kljuykov, 2013, Pl. Syst. Evol. 299 (5): 1002.

三Bunium badachschanicum Kamelin, 1977, Novit. Syst. Pl. Vasc. 14: 190.

Type: TAJIKISTAN. "Tadshikistania, Badakhshania (Pamir occidentalis), in valle fl. Gunt in vicinitate urb. Khorog, ad canalem Chonif, in schistosis, $2250 \mathrm{~m}, 14$ VII 1964, Grubov, Kurbanbekov \& Junussov" (holotype LE! [LE00051602], isotype LE! [LE00051603]).

Distribution in MA \& K: Tajikistan (Kŭhistani Badakhshan).

Distribution in other Asian countries: Afghanistan, Iran.

Distribution area: Central and South-Western Asia.

Elwendia bucharica Kljuykov et Lyskov, 2018, Phytotaxa 382(2): 214.

Type: UZBEKISTAN. "Southern side of Hissar Range, near Khojaosmin, upper reaches of Khandiza River. 07 VII 1979. Pimenov, Kljuykov, Boryaev, Baranova \& Vassilieva 483" (holotype MW [MW0595669]; isotype MW [MW0595670]).

Distribution in MA \& $\mathbf{K}$ : Uzbekistan (Surxondarya prov.). Endemic.

Distribution area: South-Western Asia.

Note: Closely related to E. capusii.

Elwendia capusii (Franch.) Pimenov et Kljuykov, 2013, Pl. Syst. Evol. 299 (5): 1002.

$\equiv$ Carum capusii Franch., 1883, Ann. Sci. Nat. Bot., sér. 6, 16: 293.

$\equiv$ Bunium capusii (Franch.) Korovin, 1927, Byull. Sredne-Asiatsk. Gosud. Univ. 15: 126.

Type: UZBEKISTAN. "Djizak. V 1881. Capus 506" (lectotype P! [P00834738]; designated by Pimenov in Bot. Zhurn. 101(10): 1229. 2016); isolectotype P [P00834739]; Schariselbs, dans les moissons. 07 V 1881. Capus 507" (syntype P! [P02496009]).

= Carum elegans auct. non Fenzl: Regel, 1878, Trudy Imp. S.-Peterburgsk. Bot. Sada 5(2): 586.
= Carum gracile V. J. Zinger, 1901, Trudy Bot. Sada Imp. Jur'evsk. Univ. 2: 162, nom. illeg., non Lindl. (1835).

Type: KAZAKHSTAN. "Riv. Keless. $13 \mathrm{~V}$ 1898. Geyer" (holotype LE! [LE00051506]).

= Carum turkestanicum Lipsky, 1904, Trudy Imp. S.-Peterburgsk. Bot. Sada 23: 113.

= Bunium turkestanicum (Lipsky) Koso-Pol., 1916, Bull. Soc. Imp. Naturalistes Moscou, n. s. 29: 194.

Type: UZBEKISTAN. "Samarkand prov., Seravschan bassin, steppa inter Ulus et Dzham. 12 V 1869. O. A. Fedtschenko" (syntype LE! [LE00051600]); "Chovast prope Samarkand. $12 \mathrm{~V}$ 1898. Paulsen 265" (syntype LE! [LE00051601]); "Promontorium Kcharli-tau. 07 V 1865. Sewerzov" (syntype LE! [LE00051599]); KAZAKHSTAN. "Karnak in Mts. Karatau, 1876, A. Regel" (lectotype LE! [LE00051597], designated by V.M. Vinogradova 1997a: 99; isolectotype LE! [LE00051598]); "Riv. Keless. 13 V 1898. Geyer" (syntype).

= Bunium gypsaceum Korovin, 1948, Not. Syst. Herb. Inst. Bot. Zool. Acad. Sci. Uzbekistan 12: 25.

Type: UZBEKISTAN. "In promontoriis gypsaceis inter urbes Bajsun et Denau, pr. fontem Chodsha-ipak, in caementis calcareis. 11 V 1930. Botschantzev \& Vvedensky 203" (holotype TASH! [TASH002291]; isotype LE! [LE00051616]).

Distribution in MA \& $\mathbf{K}$ : Kazakhstan (S: S Kazakhstan prov., Zhambyl prov.), Tajikistan (Kŭhistani Badakhshan, Sughd prov., Khatlon prov., Dushanbe prov.), Uzbekistan (Buxoro prov., Navoi prov., Dzhizak prov., Samarqand prov., Qashqadarya prov., Surxondarya prov., Sirdarya prov., Toshkent prov.), Turkmenistan (Lebap prov.). Endemic.

Distribution area: Central and South-Western Asia.

Elwendia chaerophylloides (Regel et Schmalh.) Pimenov et Kljuykov, 2013, Pl. Syst. Evol. 299 (5): 1002.

$\equiv$ Carum chaerophylloides Regel et Schmalh., 1878, Trudy Imp. S.-Peterburgsk. Bot. Sada 5(2): 585.

$\equiv$ Carum confusum O. Fedtsch., 1902, Izv. Obshch. Ljubit. Estestv. 103: 66, nom. illeg. (Art. 52.1).

$\equiv$ Bunium chaerophylloides (Regel et Schmalh.) Drude, 1898, in Nat. Pflanzenfam. (Engler et Prantl) 3(8): 194.

$\equiv$ Buniella chaerophylloides (Regel et Schmalh.) Schischk. 1960, Bot. Mater. Gerb. Bot. Inst. Komarova Akad. Nauk SSSR 20: 280. 
Type: UZBEKISTAN. "In horto prov. Fedorowii prope Taschkent, Krause" (lectotype LE!, designated by A. M. Geldykhanov 1992: 121).

= Carum sogdianum Lipsky, 1904, Trudy Imp. S.-Peterburgsk. Bot. Sada 23: 118.

= Bunium sogdianum (Lipsky) H. Wolff, 1927, in Pflanzenr. (Engler) Umbellif.-Apioid.-Ammin. 90(IV, 228): 209.

Type: UZBEKISTAN. "Inter Taschkent et Keles, 22 IV 1871, O. A. Fedtschenko" (lectotype LE! [LE00051559], designated by V. M. Vinogradova 1997a: 99); syntypes LE! [LE00051529LE00051558, LE00051560-LE00051583]).

Distribution in MA \& K: Kazakhstan (S: S Kazakhstan prov., Zhambyl prov.), Kyrgyzstan (Batken prov., Jalal-Abad prov., Osh prov.), Tajikistan (Kŭhistani Badakhshan, Sughd prov., Khatlon prov., Dushanbe prov.), Uzbekistan (Navoi prov., Samarqand prov., Qashqadarya prov., Surxondarya prov., Sirdarya prov., Toshkent prov., Namangan prov., Andijon prov.), Turkmenistan (Akhal prov.).

Distribution in other Asian countries: Afghanistan, Iran.

Distribution area: South-Western Asia.

Elwendia fedtschenkoana (Korovin ex Kamelin) Pimenov et Kljuykov, 2013, Pl. Syst. Evol. 299 (5): 1003.

$\equiv$ Bunium fedtschenkoanum Korovin ex Kamelin, 1977, Novit. Syst. P1. Vasc. 14: 191.

Type: TURKMENISTAN. "Turcomania occidentalis (Regio transcaspica), inter pagorum Karakala et Dshalilja. 13 V 1916. B. A. Fedtschenko" (holotype LE! [LE00051220]).

Distribution in MA \& K: Turkmenistan (Balkan prov., Akhal prov.).

Distribution in other Asian countries: Iran.

Distribution area: South-Western Asia.

Elwendia hissarica (Korovin) Pimenov et Kljuykov, 2013, Pl. Syst. Evol. 299 (5): 1003.

$\equiv$ Bunium hissaricum Korovin, 1948, Not. Syst. Herb. Inst. Bot.Zool. Acad. Sci. Uzbekistan 12: 27.

Type: UZBEKISTAN. "Pamir-Alaj austrooccidentalis. Montes Tadshir-kaja, in gypsaceis, 05 VI 1930, Lepeschkin" (holotype TASH! [TASH002290]).

= Bunium tenuisectum Korovin, 1975, Izv. Akad. Nauk Tadzh. SSR, Otd. Estest. Nauk 3(60): 5, nom. illeg. (Art. 53.1), non Griseb. ex Pantoc. (1873).

Type: TAJIKISTAN. "Regio meridionalis Tadzhikiae, montes Koykitau, prope p. Lublikor, alt.
600 m s. m. 18 V 1960. Junussov 1077" (holotype TAD!).

= Bunium longilobum Kljuykov, 1982, Bot. Zhurn. (Leningrad) 67(4): 544.

Type: TURKMENISTAN. "Turcomania, Badchyz, jugum Gijas-Gedyk, prope custodiam Rachmatur. 17 V 1975. Kljuykov \& Baranova 515" (holotype LE! [LE00051621]; isotype MW! [MW0593815]).

- Bunium persicum auct. non B. Fedtsch.: Kaul, 1975, J. Bombay Nat. Hist. Soc. 72(3): 708, fig. 4.

Distribution in MA \& K: Tajikistan (Khatlon prov.), Uzbekistan (Surxondarya prov.), Turkmenistan (Akhal prov., Lebap prov.).

Distribution in other Asian countries: N India, Pakistan; Afghanistan, Iran.

Distribution area: South-Western Asia.

Elwendia intermedia (Korovin) Pimenov et Kljuykov, 2013, Pl. Syst. Evol. 299 (5): 1003.

三 Bunium intermedium Korovin, 1948, Not. Syst. Herb. Inst. Bot. Zool. Acad. Sci. Uzbekistan 12: 26.

Type: KYRGYZSTAN. "Jugum Turkestanicum in valle fl. Ak-tschakmenj, in Juniperetis, $\mathrm{h}=2700$ 2800 m, 14 VII 1934, Gomolitzky \& Protopopov 140" (holotype TASH! [TASH002292]; “Jugum Turkestanicum in valle fl. Guralasch, in Juniperetum umbrosis 29 VI 1934. Zakrzewski 123" (paratype TASH)).

Distribution in MA \& K: Kazakhstan (S Kazakhstan prov.), Kyrgyzstan (Batken prov., Dzhelalabad prov., Osh prov.), Tajikistan (Kŭhistani Badakhshan, Sughd prov., Khatlon prov., Dushanbe prov.), Uzbekistan (Navoi prov., Dzhizak prov., Samarqand prov., Qashqadarya prov., Surxondarya prov., Toshkent prov.), Turkmenistan (Lebap prov.).

Distribution in other Asian countries: Afghanistan, Iran.

Distribution area: South-Western Asia.

Elwendia kopetdaghensis (Geld.) Pimenov et Kljuykov, 2013, Pl. Syst. Evol. 299 (5): 1003.

$\equiv$ Bunium kopetdaghense Geld. 1985, Bot. Zhurn. (Leningrad) 70(11): 1559.

Type: TURKMENISTAN. "Turcomania, in declivius collium, solum subargillosum, SW margo oppidi Aschchabad. 21 V 1952. Tzvelev" (holotype LE! [LE00051617]; isotype MW [MW0593814]).

Distribution in MA \& K: Turkmenistan (Balkan prov., Akhal prov.). Endemic.

Distribution area: South-Western Asia. 
Elwendia kuhitangi (Nevski) Pimenov et Kljuykov, 2013, Pl. Syst. Evol. 299 (5): 1003.

三Bunium kuhitangi Nevski, 1937, Trudy Bot. Inst. Akad. Nauk SSSR (ser. 1 Fl. Sist. Vyssh. Rast.) 4: 274 .

Type: TURKMENISTAN. "Ad declivia argillosa lapidosa sub cacuminibus montiumi Kuhitang, supra pagum Chodzha-fil-ata. 27 VI 1931, Nevski 462" (holotype LE! [LE00051620]).

Distribution in MA \& K: Tajikistan (Kŭhistani Badakhshan, Dushanbe prov.), Uzbekistan (Surxondarya prov.), Turkmenistan (Lebap prov.). Endemic. May be found in adjacent part of Afghanistan.

Distribution area: South-Western Asia.

Note: The assignment Bunium longipes var depressum, which occurs in the central part of Mts. Kopetdag, in synonymy of this species is mistaken.

Elwendia latiloba (Korovin) Pimenov et Kljuykov, 2013, P1. Syst. Evol. 299 (5): 1003.

三 Bunium latilobum Korovin, 1973, Izv. Akad. Nauk Tadzh. SSR, Otd. Estest. Nauk 2(51): 10.

Type: TAJIKISTAN. "Tadshikistania, pars meridionalis montes Koyki-Tau, in regione pag. Lublikor. 18 V 1960. Junussov 1036" (holotype TAD!, probably lost). Neotype from Koyki-tau (Pimenov et al., 656 [MW0859178]) has been recently proposed (Kljuykov et al., 2018).

Distribution in MA \& K: Tajikistan (Khatlon prov.), Uzbekistan (Qashqadarya prov., Surxondarya prov.). Endemic.

Distribution area: South-Western Asia.

Elwendia longipes (Freyn) Pimenov et Kljuykov, 2013, P1. Syst. Evol. 299 (5): 1003.

三Bunium longipes Freyn, 18961906, Bull. Herb. Boissier (sér. 2) 6: 214.

三Bunium cylindricum (Boiss. et Hohen.) Freyn subsp. longipes (Freyn) H. Wolff, 1927, in Pflanzenr. (Engler) Umbellif.-Apioid.-Ammin. 90 (IV, 228): 193.

Type: TURKMENISTAN. "Ashkhabad, in graminosis siccis ad Gjaurs, 27 IV 1900, Sintenis 169b" (lectotype LE! [LE00051622], designated by A. M. Geldykhanov 1985a: 1564); isolectotypes LD! [LD1309054, LD1405197], P! [P00834749], W [W0053736 - fragm.]); "Karakala, in herbedis. $17 \mathrm{~V}$ 1901. Sintenis 1737a" (syntypes JE [JE00003866], LD! [LD1362079], W [W0053734 - fragm.]).

= Bunium cylindricum (Boiss. et Hohen.) Freyn var. minor Freyn, 1906, Bull. Herb. Boissier (sér. 2) 6: 214, "cylindraceum".
= Bunium longipes Freyn subsp. minor (Freyn) Geld., 1985, Bot. Zhurn. (Leningrad) 70 (11): 1564.

Type: TURKMENISTAN. "Kisil-Arwat, in montosis. 05 V 1901. Sintenis 1629" (lectotype LE! [LE00051614], designated by A. M. Geldykhanov 1985: 1564); isolectotypes LD!, STU); “Aschabad, in prat. subalpinis montis Tangeri Gargan. $04 \mathrm{~V}$ 1900. Sintenis 231" (syntypes B! [B 10 0278970], K [K000685688], LD! [LD1315534, LD1412938, LD1412998]).

= Bunium cylindricum (Boiss. et Hohen.) Freyn var. brachycarpum Freyn, 1906, Bull. Herb. Boissier (sér. 2) 6: 214, "cylindraceum".

Type: TURKMENISTAN. "Regio Transcaspica, Chodschakala, in steppes. 14 V 1901. Sintenis 1738" (lectotype LD! [LD1315519]; designated by Pimenov et Sutorý (2014: 1104).

= Bunium longipes Freyn var. ellipsoideum Freyn, 18961906, Bull. Herb. Boissier (sér. 2) 6: 215.

Type: TURKMENISTAN. "Karakala, in herbidis. 17 V 1901. Sintenis 1737b" (holotype LD! [LD1386038]).

= Bunium longipes Freyn var. depressum Korovin, 1927, Byull. Sredne-Asiatsk. Gosud. Univ. 15: 124 .

= Bunium korovinii Kamelin et Geld. 1985, Bot. Zhurn. (Leningrad) 70(11): 1562.

Type: TURKMENISTAN. "Turcomania, Kopetdag centralis, in ascensu ad cacuminem Czopandagh. $14 \mathrm{~V}$ 1928. B. A. Fedtschenko, Bobrov, Jarmolenlo \& Gontscharow 670" (holotype LE! [LE00051618; isotype LE! [LE00051619]).

Distribution in MA \& K: Turkmenistan (Balkan prov., Akhal prov.).

Distribution in other Asian countries: Afghanistan, Iran.

Distribution area: South-Western Asia.

Elwendia persica (Boiss.) Pimenov et Kljuykov, 2013, P1. Syst. Evol. 299 (5): 1004.

$\equiv$ Carum persicum Boiss., 1844, Ann. Sci. Nat. sér. 3, 1: 138, nom. cons. prop. (Art. 14).

$\equiv$ Bunium persicum (Boiss.) B. Fedtsch., 1915, Rastit. Turkest.: 612.

Type: IRAN. "Persia australis, Aucher-Eloy 4555" (lectotype G-BOIS! [G00359481], designated by A. M. Geldykhanov (1992: 122), as "type", and by Pimenov (2017: 762); isolectotypes G [G00359482], K! [K000685695], P! [P00834751, P00834752], US! [US00588543]); "Inter Fasa et Chyraz, Aucher-Eloy 4553" (syntypes G! [G00359482], P! [P00834750]). 
= Sium cyminosma Basiner, 1843, in Fischer et C. A. Meyer (eds.), Index Sem. Horti Petrop. 9, Suppl.: 18 , nom rejic.

= Pimpinella cyminosma (Basiner) Koso-Pol., 1916, Bull. Soc. Imp. Naturalistes Moscou, n. s. 29: 180.

Type: UZBEKISTAN. "Seeds: Col. Chivensibus sub nomina ssara[e]-sir, 1843, Basiner" (lectotype LE! [LE01065721]; designated by Pimenov in Taxon 66(3): 762. 2017; isolectotype P!).

$=$ Carum heterophyllum Regel et Schmalh., 1878, Trudy Imp. S.-Peterburgsk. Bot. Sada 5, 2: 586.

Type: TAJIKISTAN. "Zeravschan, inter Varsaminor et Peti, 4500-6000', 12 VI 1870, O. A. Fedtschenko" (lectotype LE! [LE00051507], designated by V. M. Vinogradova 1997a: 98).

Distribution in MA \& K: Kyrgyzstan (Batken prov., Jalal-Abad prov., Osh prov.), Tajikistan (Kŭhistani Badakhshan, Sughd prov., Khatlon prov., Dushanbe prov.), Uzbekistan (Buxoro prov., Navoi prov., Dzhizak prov., Samarqand prov., Qashqadarya prov., Surxondarya prov., Toshkent prov.), Turkmenistan (Balkan prov., Akhal prov.).

Distribution in other Asian countries: Chana, Pakistan, Afghanistan, Iran.

Distribution area: Central and South-Western Asia.

Elwendia salsa (Korovin) Pimenov et Kljuykov, 2013, Pl. Syst. Evol. 299 (5): 1004.

三Bunium salsum Korovin, 1924, Bot. Mater. Gerb. Glavn. Bot. Sada R.S.F.S.R. 5, 6: 86.

Type: UZBEKISTAN. "In prov. Samarkand, prope urb. Samarkand, ad colles Tschupan-Ata. 03 V 1916. Betger" (syntype TASH [TASH002295]); "Samarkand, Tschupan-ata, 04 VI 1921, Popov 215" (lectotype TASH! [TASH002297], designated by Pimenov et Kljuykov 2002: 115), isolectotype TASH! [TASH002296]).

- Bunium cylindricum auct. non Drude: Korovin, 1950, in Schischkin (ed.), Fl. URSS 16: 411, p. p.

Distribution in MA \& K: Kyrgyzstan (Batken prov.), Tajikistan (Sughd prov.), Uzbekistan (Dzhizak prov., Navoi prov., Samarqand prov., Qashqadarya prov., Sirdarya prov.). Endemic.

Distribution area: South-Western Asia.

Note: The description of this species, as well as of other species in Korovin (1924a) does not contain material for species typification. This is right also for Korovin's publications of 1923 and some subsequent years.
Elwendia sary-cheleki (Lazkov et Kljuykov) Pimenov et Kljuykov, 2013, P1. Syst. Evol. 299 (5): 1004.

$\equiv$ Bunium sary-cheleki Lazkov et Kljuykov, 2011, Bot. Zhurn. (St. Perersburg) 96(7): 967.

Type: KYRGYZSTAN. "Jugum Chatkalicum, ripa sinistra fluvii Chodscha-Ata, in viciniis pagi Arkit, in saxosis, $1250 \mathrm{~m} \mathrm{s.} \mathrm{m.} 41^{\circ} 47^{\prime} 02.4^{\prime \prime}$ lat. bor., $71^{\circ} 57^{\prime}$ long. or. 04 VI 2010. Lazkov" (holotype LE! [LE00051623]; isotypes FRU, LE! [LE00051625], MW! [MW0593826]).

Distribution in MA \& K: Kyrgyzstan (JalalAbad prov.). Endemic.

Distribution area: South-Western Asia.

Elwendia seravschanica (Korovin) Pimenov et Kljuykov, 2013, Pl. Syst. Evol. 299 (5): 1004.

三Bunium seravschanicum Korovin, 1927, Byull. Sredne-Asiatsk. Gosud. Univ. 15: 125.

Type: TAJIKISTAN. "Asia Media, Prov. Samarkand, Semurutsch inter Kschtut et lacum Kul-i Kalon, 8-9000'. 21 VI-03 VII 1882. A. Regel" (lectotype LE!; designated by Kljuykov et al., 2018: 218).

Note: Lectotype, previously selected by Vinogradova, 1997a: 97 ("Buchara, prov. Rochan supra cast. Kala-i-Vamir in ascensu, faucium Odidi in lapidosis, 8700-9000', 10 VIII 1901, Alexeenko" (lectotype TASH!, designated by Korovin 1950b: 405; isolectotype LE!)), did not origin from Zevavschan basin as Rochan is a locality from Badakhshan. Kljuykov et al. (2018) stated morphological difference between Rochan plants and those from Seravschan basin as the former have subglobose fruits, shorter stylods and weekly refracted leaf blades. They believed that lectotype, previously selected by V. M. Vinogradova (1997a: 97), which differs from Zeravshan plants, could be attributed to another species. In Tashkent herbarium (TASH) no duplicates of Alexeenko's gatherings from Rochan were found.

Distribution in MA \& K: Tajikistan (Sughd prov.), Uzbekistan (Dzhizak prov., Qashqadarya prov., Samarqand prov.). Endemic.

Distribution area: South-Western Asia.

Elwendia setacea (Schrenk) Pimenov et Kljuykov, 2013, Pl. Syst. Evol. 299 (5): 1004.

$\equiv$ Carum setaceum Schrenk, 1841, Enum. P1. Nov. 1: 61. 
$\equiv$ Conopodium setaceum (Schrenk) Korovin, 1924, Byull. Sredne-Asiatsk. Gosud. Univ. 7, Supp1. (Sched. Herb. Fl. As. Med. 1-2): 24.

$\equiv$ Bunium setaceum (Schrenk) H. Wolff, 1927, in Pflanzenr. (Engler) Umbellif.-Apioid.-Ammin. 90(IV, 228): 209.

EScaligeria setacea (Schrenk) Korovin, 1928, Byull. Sredne-Asiatsk. Univ. (ser. 8b, Bot.) 2: 67.

Type: KAZAKHSTAN. "In fruticetis collium Songoriae ad rivulum $\mathrm{Ai}$, hinc inde, in montibus Alatau ad fl. Lepsa et Baskan [Songarei, in campis versus montes Arganaty], 01 1840, Schrenk" (lectotype LE! [LE00051517], designated by V. M. Vinogradova 1997a: 98; isolectotypes LE! [LE00051518, LE00051519, LE00051520, LE00051522, LE00051523]).

= Carum capillifolium Kar. et Kir. 1841, Bull. Soc. Imp. Naturalistes Moscou 14: 428.

= Bunium capillifolium auct. non Bertol.: Botsch. 1976, Novit. Syst. Pl. Vasc. 13: 249.

Type: KAZAKHSTAN. "In fruticetis collium Soongoriae ad rivulum Ai, hinc inde, in montosis Alatau ad fl. Lepsa et Baksan, 1841, Karelin \& Kirilov 1531" (neotype MW! [MW0593811, MW0593812, MW0593813], designated by Kljuykov et al. 1977: 143); isoneotypes LE! [LE00051609-LE00051612], M! [M0172956]).

$=$ Carum setaceum Schrenk var. borgatense Lipsky, 1904, Trudy Imp. S.-Peterburgsk. Bot. Sada 23: 126

Type: CHINA. "Kuldsha, fliv. Borgaty (Kasch). 08 VII 1879. A. Regel" (holotype LE!).

Distribution in MA \& K: Kazakhstan (NE: E Kazakhstan prov.; Central: Karagandy prov., KzylOrda prov.; S: S Kazakhstan prov., Zhambyl prov.; SE: Almaty prov.), Kyrgyzstan (Jalal-Abad prov., Ysyk-köl prov., Naryn prov., Osh prov., Talas prov., Chüh prov.), Uzbekistan (Toshkent prov.), Turkmenistan (Akhal prov.).

Distribution in other Asian countries: China, Mongolia.

Distribution area: Central and South-Western Asia.

Elwendia ugamica Kljuykov et Lyskov, 2018, Phytotaxa 382 (2): 219.

Type: UZBEKISTAN. "Western Tian-Shan, southern side of Ugam Range, upper course of Alikhodja River. 17 VI 1962. Adylov 1882" (holotype TASH).

Distribution in MA \& K: Uzbekistan (Toshkent prov.). Endemic.
Distribution area: South-Western Asia.

Note: The species is closely related to E. capusii.

Elwendia vaginata (Korovin) Pimenov et Kljuykov, 2013, Pl. Syst. Evol. 299 (5): 1004.

$\equiv$ Bunium vaginatum Korovin, 1927, Byull. Sredne-Asiatsk. Gosud. Univ. 15: 122, tab. 6.

Type: KAZAKHSTAN. "Montes Karatau, Kulan-tau, prop. fluv. Kulan. 28 V 1922. Drobov 90" (lectotype TASH! [TASH003703]; designated here; "ibidem, in valle fl. Kok-tal. 02 VI 1925. Sovetkina" (syntype TASH); "in mont. Ak-tau. 16 VI 1925. Sovetkina" (syntype TASH)).

Distribution in MA \& K: Kazakhstan (S: S Kazakhstan prov., Zhambyl prov.), Uzbekistan (Toshkent prov.). Endemic.

Distribution area: South-Western Asia.

Note: In the description of the species Korovin (1927a: 122) lists three collections (Drobov, Kulantau, and Sovetkina, Kok-tal and Alatau; all from the Syrdarya Karatau). No specimen is marked as holotype. In treatment for the genus for "Flora of the URSS" Korovin (1950: 400) indicated that the type material is in Tashkent.

Eremodaucus lehmannii Bunge, 1843, Del. Sem. Hort. Dorp.: 6; ejusd. 1844, Linnaea 18: 6 et 151.

三 Trachydium lehmannii (Bunge) Benth., 1867, in Gen. Pl. (Bentham et Hooker f.) 1: 884.

Type: UZBEKISTAN. "In regione Katta-Kurgan prope Bucharam, VIII 1841, Lehmann [567]" (lectotype LE! [LE00050133]; designated by V. M. Vinogradova 2002: 139; isolectotypes K! [K000697395], P! [P02422176, P02422177], W [W0057143]).

= Albertia margaritifera Regel et Schmalh., 1878, Trudy Imp. S.-Peterburgsk. Bot. Sada 5(2): 605.

Type: UZBEKISTAN. "In deserto inter Taschkent et fluviam Syr-Darja. $20 \mathrm{~V}$ 1871. O. A. Fedtschenko" (lectotype LE! [LE00051315], designated by V. M. Vinogradova 1999: 81; isolectotype LE [LE00051313, LE00051314]); "In Kokania, in valle fluvii Sarawschan, O. A. Fedtschenko" (syntype LE!); "Karnak, Karatau 1876. A. Regel" (syntype LE [LE00051312]; "Syr-Darja. Golike" (syntype LE [LE00051311]; "Fort Turkestan 05.1874. Golike" (syntype LE); "Steppe in W foodhill of Mogol-Tau. Sewertzow" (syntype LE [LE00051309]; "In montibus Karatau. 1882. Krause" (syntype LE [LE00051307]). 
Distribution in MA \& K: Kazakhstan (S: S Kazakhstan prov., Zhambyl prov.; SE: Almaty prov.), Kyrgyzstan (Batken prov., Jalal-Abad prov., Osh prov., Talas prov., Chüh prov.), Tajikistan (Kŭhistani Badakhshan, Sughd prov., Khatlon prov., Dushanbe prov.), Uzbekistan (Buxoro prov., Navoi prov., Dzhizak prov., Samarqand prov., Qashqadarya prov., Surxondarya prov., Sirdarya prov., Toshkent prov., Fergona prov., Andijon prov.), Turkmenistan (Balkan prov., Akhal prov., Lebap prov., Mary prov.).

Distribution in other Asian countries: Afghanistan, Iran, Azerbaidzhan.

Distribution area: Central and South-Western Asia.

Eriosynaphe longifolia (Fisch. ex Spreng.) DC., 1829, Col. Mém. 5 (Mém. Fam. Ombell.): 175.

$\equiv$ Ferula longifolia Fisch. ex Spreng., 1818, Sp. Umbell.: 86.

$\equiv$ Peucedanum longifolium (Fisch. ex Spreng.) Baill. 1879, Hist. P1. (Baillon) 7: 186, nom. illeg. (Art. 53.1), non Waldst. et Kit. (1812).

$\equiv$ Johrenia longifolia (Fisch. ex Spreng.) Calest. 1905, Webbia 1: 224.

EEriosynaphe cachroides Koso-Pol., 1916, Bull. Soc. Imp. Naturalistes Moscou, n. s. 29: 127, nom. illeg. (Art. 52.1).

Type: EUROPE, RUSSIA. "Ad Sareptam, 1803. coll. unknown” (lectotype B-Willd! [B-W 05771-01 0, B-W 05771-02 0]; designated here by Pimenov; isolectotypes LE-Bieb!, PH [PH00042588]).

Distribution in MA \& K: Kazakhstan (W: W Kazakhstan prov., Atyrau prov., Aktobe prov.).

Distribution in other Asian countries: Russia.

Distribution area: Asia (N, Central), Europa.

Eryngium billardierei F. Delaroche, 1808, Nouv. Bull. Sci. Soc. Phylom. Paris: 25, tab. 2.

Type: SYRIA/LEBANON. "Syrie-Leban. Labillardière" (holotype G-Hb. Delessert [G00367550]; designated by A. Wörz, 2011: 148; isotype FI [FI004401]).

= Eryngium noёanum Boiss., 1856, Diagn. P1. Orient. (ser. 2) 2: 72.

Type: IRAN. "Prope Kirrind in Persiâ Mesopotamica finitimâ [Kirrind in Persia]. IX 1851. Noё 1112" (holotype G-BOIS!; designaited by Pimenov \& Tamamschjan (1987: 53); Pimenov \& Jacquemoud (2020: 6)).

$=$ Eryngium nigromontanum Boiss. et Buhse, 1860, Nouv. Mém. Soc. Imp. Naturalistes Moscou 12: 95 .
= Eryngium billardierei F. Delaroche subsp. nigromontanum (Boiss. et Buhse) H. Wolff, 1913, in Pflanzenr. (Engler) Umbell. - Sanicul. 61(IV, 228): 150.

Type: IRAN. "In Karadagh [In montosis ad Nemedabad prope Tabris in ditione Aderbidjan], 07 VII 1847, Buhse" (lectotype LE!, designated by A. Wörz (2011: 148; isolectotype G-BOIS, n. v.).

= Eryngium billardierei F. Delaroche var. meiocephalum Boiss, 1872, Fl. Orient. (Boissier) 2: 825.

Type: IRAN. "Montes Avroman et Schahu. Alt. 6000'-8000', VII 1867, Haussknecht 448" (lectotype G-BOIS!: designated by A. Wörz 2011: 148).

= Eryngium noёanum Boiss. var. integrifolium Boiss., 1872, Fl. Orient. (Boissier) 2: 824.

Type: IRAN. "In Persiae austro-orientalis monte Dalechani supra Sungur. [In gram. calc. m. Dalechani supra Sungur, 6000', 1867, Haussknecht $448 c$ " (holotype G-BOIS!).

= Eryngium orientale Stapf et Wettst. 1886, Denkschr. Akad. Wien 51: 315, nom. illeg. (Art. 53.1), non Mill. (1768).

Type: IRAN. "In locis siccis lapidosis in montibus prope Hamadan, 04 VIII 1882, Polak \& Pichler" (holotype WU! [WU0073151]).

= Eryngium polycephalum Hausskn. ex H. Wolff, 1913, in Pflanzenr. (Engler) Umbellif.-Apioid.Ammin. 61(IV, 228): 153.

Type: TURKEY. "[Adiyaman] Auf dem AkDagh, zwischenAdiaman und Malatia, Haussknecht" (lectotype G-BOIS; designated by A. Wörz 2011: 326; isolectotype JE! [JE00003932]).

= Eryngium nigromontanum Boiss. et Buhse var. simplicatum Bordz. 1934, Zhurn. Inst. Bot. Vseukrajinsk. Akad. Nauk 3: 73.

Type: AZERBAIDZHAN. "Aserbajdzan, Respublica Nakhiczewanica. In monte Kassabadagh in vicinis pagi Karabagljar, Lorens" (holotype KW?).

= Eryngium balchanicum Bobrov, 1950, in Schischkin (ed.), Fl. URSS 16: 589, 79, tab. 4, fig. 4.

Type: TURKMENISTAN. "Balchany Majores, promontorium australe prope fontem Berk-tscheme dicto non procul a statione viae ferreae Dzhebel, 03 VI 1928, Bobrov \& Jarmolenko" (lectotype LE! [LE00050996]; designated as holotype by V. M. Vinogradova (1997a: 99); isolectotypes [LE00050995, LE00050997]).

- Eryngium campestre auct. non L.: B. Fedtsch., 1909, Consp. Fl. Turkest. (O. A. Fedtschenko \& B. A. Fedtschenko) 3: 78. 
- Eryngium amethystinum auct. non L.: M. Hiroe, 1979, Umbelliferae World: 1856, p. p.

Distribution in MA \& K: Turkmenistan (Balkan prov., Akhal prov.).

Distribution in oth er Asian countries: India, Pakistan, Afghanistan, Iran, Azerbaidzhan, Armenia, Turkey, Iraq, Lebanon, Syria, Israel.

Distribution area: Central, South-Western \& Mediterranean Asia

Eryngium bungei Boiss., 1872, Fl. Orient. (Boissier) 2: 824.

Type: IRAN. "In Persiae prov. Khorassan, prope Riwed inter Schahrud et Nischapur, VI 1858, Bunge" (lectotype G-BOIS!; designated as "typus" by Pimenov \& Tamamschjan (1987: 53); A. Wörz 2011: 176; isolectotypes LE! [LE00015640], P [P00834259, P00834260, P00834301]).

= Eryngium kermanense Bornm. 1906, Bull. Herb. Boissier (2) 6: 766, nom. inval (Art. 36.1).

- Eryngium macrocalyx auct. non Schrenk: O. et B. Fedtsch., 1909, Consp. Fl. Turkest. (O. A. Fedtschenko et B. A. Fedtschenko) 3: 78, p. p.

Distribution in MA \& K: Turkmenistan (Balkan prov., Akhal prov., Mary prov.).

Distribution in other Asian countries: Afghanistan, Iran. Asia.

Distribution area: Central and South-Western

Eryngium caeruleum M. Bieb. 1798, Tabl. Prov. Mer., 1800, Beschr. Casp.: 112.

$\equiv$ Eryngium biebersteinianum Nevski, 1937, Trudy Bot. Inst. Akad. Nauk SSSR (ser. 1 Fl. Sist. Vyssh. Rast. 4): 275.

Type: AZERBAIDZHAN. "Shirvan Steppe [Ex montibus Schirvanensibus]. Marschall von Bieberstein (?)" (lectotype LE!, designated as "typus" by Pimenov \& Tamamachjan (1987: 54); isolectotype B-Willd. [B-W 05574-01 0]; GEORGIA. "In Iberia circa Tiflis, 1804, Steven (?)" (syntypes LE!, MW).

= Eryngium caucasicum Trautv. 1871, Trudy Imp. S.-Peterburgsk. Bot. Sada 1: 23.

Type: RUSSIA. "Prope Derbent, 1870, Becker 191" (lectotype LE!, designated by A. Wörz 2011: 180 and Pimenov in Pimenov et Ostroumova 2012: 44); GEORGIA. "In Cartalinia, Imeretia et Mingrelia, Lagovsky" (syntype LE!); "In Abchasia, Lagovsky" (syntype LE!).

= Eryngium caeruleum M. Bieb. var. fallax Woronow, 1908, Vestn. Tiflissk. Bot. Sada 10: 8.

Type: GEORGIA. "Tiflisskaja gub., Lagodechi, Purin, Mlokosevich" (syntypes); AZERBAI-
DZHAN. "Bakinskaja gub., Lenkoran: Asakudzha, Lomakin" (syntype TBI [TBI1024255]); "Ins. Sara, Kizeritski" (syntype).

= Eryngium pskemense Pavlov, 1950, Vestnik Akad. Nauk Kazak. SSR 3 (60): 30.

Type: UZBEKISTAN. "Prov. Austro-Kazachstania, distr. Bostandyk, in decliviis stepposis prope fl. Pskem ad rur. Bogustan, 11 VI 1948, N. V. Pavlov 201" (lectotype AA!: designated by V. P. Goloskokov (1963: 290); isolectotypes MW! [MW0593731, MW0593032]); "In declivibus stepposis australis faucem Nauvali-ssaj (vall. fl. Pskem), 15 VII 1948, Bykov" (syntypes AA!, MW!).

Distribution in MA \& K: Kazakhstan ( $\mathrm{S}$ : S Kazakhstan prov.), Kyrgyzstan (Batken prov., Jalal-Abad prov.), Tajikistan (Sughd prov., Khatlon prov., Dushanbe prov.), Uzbekistan (Buxoro prov., Dzhizak prov., Samarqand prov., Qashqadarya prov., Surxondarya prov., Sirdarya prov., Toshkent prov.), Turkmenistan (Balkan prov., Akhal prov., Lebap prov., Mary prov.).

Distribution in other Asian countries: Russia, Pakistan, Afghanistan, Iran, Azerbaidzhan,

Georgia, Armenia, Turkey.

Distribution area: Central and South-Western Asia, Europa.

Note: V. P. Goloskokov (1963) noted that the type of species name in AA may be lost. In this case, the neotype can be selected from the isotypes stored in MW.

Eryngium karatavicum Iljin, 1936, Repert. Spec. Nov. Regni Veg. 39: 320.

Type: KAZAKHSTAN. "Montes Karatau, declivia schistosa ad fl. Koldenensai, prope summo, 07 VIII 1934, Iljin \& Iljina 19" (lectotype LE! [LE0001000]; designated as holotypus by V. M. Vinogradova (1999: 86); isolectotype LE (LE00050999).

Distribution in MA \& K: Kazakhstan (S: S Kazakhstan prov., Zhambyl prov.). Endemic.

Distribution area: South-Western Asia.

Eryngium macrocalyx Schrenk, 1841, Enum. Pl. Nov. 1: 60.

Type: KAZAKHSTAN. "In desertis Songariae, ad rivulum Kinasch [Songaria, in deserti ad fl. Kunasch], 17 VII 1840, Schrenk" (lectotype LE! [LE00051005]; designated as holotypus by V. M. Vinogradova (1999: 86); isolectotypes LE [LE00051002, LE00051003, LE00051004, LE00051006, LE00051007], MANCH!).

= Eryngium incognitum Pavlov, 1938, Byull. Moskovsk. Obshch. Isp. Prir. n. s. 47(1): 81. 
Type: TAJIKISTAN. "Tadshikistania, in collibus prope Stalinabad [Dushanbe], $1000 \mathrm{~m}$ alt., $08 \mathrm{VII}$ 1932, Stschukin" (holotype MW! [MW0593729]).

= Eryngium pamiroalaicum Korovin, 1947, Not. Syst. Herb. Inst. Bot. Zool. Acad. Sci. Uzbekistan 8: 3.

Type: TAJIKISTAN. "Pamir-Alaj meridionalis, montes Baba-tag prope p. Dshida-bulak, 03 VII 1936, Lepeschkin \& Muchamedshanov 389" (holotype TASH! [TASH002169]; "Pamir-Alai meridionalis, in collibus inter urbes Denau et Baissun. 20 V 1930. Botschantzev \& Vvedensky 291" (paratype TASH)).

- Eryngium polycephalum auct. non Hausskn. ex H. Wolff: Rech. f. et Riedl, 1963., in Biol. Skr. 13, 4 (Symb. Afghan. 5): 56.

Distribution in MA \& K: Kazakhstan (S: S Kazakhstan prov., Zhambyl prov.; SE: Almaty prov.), Kyrgyzstan (Batken prov., Jalal-Abad prov., Ysykköl prov., Naryn prov., Osh prov., Talas prov., Chüh prov.), Tajikistan (Kŭhistani Badakhshan, Sughd prov., Khatlon prov., Dushanbe prov.), Uzbekistan (Andijon prov., Dzhizak prov., Qashqadarya prov., Samarqand prov., Surxondarya prov., Toshkent prov.).

Distribution in other Asian countries: China, Afghanistan.

Distribution area: Central \& South-Western Asia.

Eryngium mirandum Bobrov, 1950, in Schischkin (ed.), Fl. URSS 16: 589.

Type UZBEKISTAN. "Karakalpakia, in monte Beltau, 21 VI 1932, Muravliansky" (holotype LE! [LE00050994]).

Distribution in MA \& K: Uzbekistan (Karakalpak). Endemic.

Distribution area: South-Western Asia.

Note: A. Wörz (2011) regarded the species name as a synonym of E. octophyllum Korovin

Eryngium octophyllum Korovin, 1947, Not. Syst. Herb. Inst. Bot. Zool. Acad. Sci. Uzbekistan 8: 3 .

Type: UZBEKISTAN. "Pamir-Alaj, vallis Zeravschan, prope pag. Urgut, 27 VI 1936, Gnezdillo" (holotype TASH! [TASH002168]).

Distribution in MA \& K: Kazakhstan (S: S Kazakhstan prov.), Kyrgyzstan (Jalal-Abad prov.?), Tajikistan (Sughd prov.), Uzbekistan (Buxoro prov., Dzhizak prov., Qashqadarya prov., Samarqand prov.), Endemic.

Distribution area: Central and South-Western Asia.
Eryngium planum L., 1753, Sp. P1.: 233.

Type: EUROPE. "[Danube near Vienna, and. Silesia]? Herb. Clifford, 87, Eryngium 1, Fol. A" (lectotype BM-Cliff [BM000558217], designated by Jury \& Southam in Jarvis et al. 2006: 212).); Leiden, van Royen (syntype L! [L0053011]).

Distribution in MA \& K: Kazakhstan (W: W Kazakhstan prov., Aktobe prov., Mangystau prov.; $\mathrm{N}$ : Kostonai prov., N Kazakhstan prov., Akmola prov., Pavlodar prov.; NE: E Kazakhstan prov.; Central: Karagandy prov.; S: Zhambyl prov.; SE: Almaty prov.).

Distribution in other Asian countries: Russia, China, Mongolia, Georgia.

Distribution area: Northern, Central and SouthWestern Asia, Europa, N. America.

Falcaria vulgaris Bernh., 1800, Syst. Verz. (Bernhardi): 176, nom. cons.

$\equiv$ Sium falcaria L., 1753, Sp. P1.: 252.

$\equiv$ Seseli falcaria Crantz, 1767, Cl. umbell. emend.: 91.

$\equiv$ Drepanophyllum sioides Wibel, 1799, Prim.

Fl. Werth.: 196, nom. illeg. (Art. 52.1).

$\equiv$ Prionitis falcata Delarbre, 1800, Fl. Auvergne (ed. 2): 421, nom. illeg. (Art. 52.1).

$\equiv$ Bunium falcaria M. Bieb., 1808, Fl. Taur.Cauc. 1: 211.

$\equiv$ Drepanophyllum agreste Hoffm., 1814, Gen.

P1. Umbell.: 109. nom. illeg. (Art. 52.1).

$\equiv$ Critamus agrestis Besser, 1822, Enum. pl. (Besser): 93. nom. illeg. (Art. 52.1).

$\equiv$ Falcaria rivinii Host, 1827, Fl. austriaca 1: 381. nom. illeg. (Art. 52.1).

$\equiv$ Prionitis falcaria Dumort., 1827, Fl. Belg. (Dumortier): 77.

$\equiv$ Drepanophyllum falcaria Desv., 1827, Fl. Anjou: 248.

$\equiv$ Falcaria agrestis (Hoffm.) Sweet, 1830, Hort. Brit. (ed. 2): 245.

$\equiv$ Critamus falcaria Rchb. 1827, Moessl. Handb. Gewächsk. (ed. 2) 1: 478.

$\equiv$ Falcaria sioides (Wibel) Asch., 1864, Fl. Brandenburg: 241, nom. illeg. (Art. 52.1).

$\equiv$ Carum falcaria (L.), 1874, Prodr. Fl. Hispan. 3: 92.

$\equiv$ Selinum falcaria E. H. L. Krause, 1904, in Deutsch. Fl. (Sturm) ed. 2 12: 45, tab. 7.

Type: EUROPE. "In Flandria, Helvetia, Bohemia, Alsatia, Gallia, Herb. Clifford, 98, Sium 5" (lectotype BM-Cliff [BM000558309], designated by Rechinger 1987: 306). 
$=$ Falcaria vulgaris Bernh. f. persica $($ Stapf et Wettst.) Bornm., 1915, Beih. Bot. Centralbl. 32(2): 241.

= Falcaria persica Stapf et Wettst., 1886, Denkschr. Akad. Wien 51: 316.

$=$ Falcaria rivinii Host var. persica $($ Stapf et Wettst.) Bornm., 1915, Beih. Bot. Centralbl. 32(2): 391.

Type: IRAN. "Ad agros circa Haydere prope Hamadan, 28 VII - 01 VIII 1882 Polak \& Pichler" (syntypes W [W 1904-0002729], WU [WU0073011WU007315]).

Distribution in MA \& K: Kazakhstan (W: W Kazakhstan prov., Aktobe prov., Mangystau prov.; N: Kostonai prov., Akmola prov; NE: E Kazakhstan prov.; Central: Karagandy prov., S: S Kazakhstan prov., Zhambyl prov.; SE: Almaty prov.), Kyrgyzstan (Batken prov., Jalal-Abad prov., Osh prov.), Tajikistan (Sughd prov., Khatlon prov., Dushanbe prov.), Uzbekistan (Buxoro prov., Navoi prov., Dzhizak prov., Samarqand prov., Qashqadarya prov., Surxondarya prov., Sirdarya prov., Toshkent prov.), Turkmenistan (Balkan prov., Akhal prov., Mary prov.)

Distribution in other Asian countries: Russia, India, Afghanistan, Iran, Azerbaidzhan, Georgia, Armenia, Turkey, Iraq, Lebanon, Syria, Israel, Jordan, Cyprus.

Distribution area: Northern: N, Central, SouthWestern \& Mediterranean Asia, Europa, Africa, N America (adv.)

Fergania polyantha (Korovin) Pimenov, 1982, Novit. Syst. P1. Vasc. 19: 120.

$\equiv$ Ferula polyantha Korovin, 1947a, Ill. Monogr. Ferula: 55.

$\equiv$ Peucedanum polyanthum (Korovin) Korovin, 1951, in Schischkin (ed.), Fl. URSS 17: 202.

Type: KYRGYZSTAN. Prov. Fergana, distr. Skobelev, area Anchor, pharangium Arpa, declivum argilloso-schistosum in pars media. $21 \mathrm{~V} 1916$. Drobov 1013 (holotype LE! [LE00051879]; isotype TASH! [TASH002477]).

Distribution in MA \& K: Kyrgyzstan (Batken prov., Osh prov.), Tajikistan (Sughd prov.), Uzbekistan (Fergona prov.). Endemic.

Distribution area: South-Western Asia.

Ferula akitschkensis B. Fedtsch. ex KosoPol., 1925, Byull. Obshch. Estestvoisp. Voronezsk. Gosud. Univ. 1: 94.

Type: KAZAKHSTAN. "Prov. Heptapotamica, jugum Dzhungarsky Alatau, prope stat. Ak-Itschke, steppa, 20 VII 1908, B. A. Fedtschenko 1633" (lectotype LE! [LE01065824, LE01065825]; designated here; isolectotypes LE! [LE01065825LE010065833]).

$=$ Ferula transitoria Korovin, 1947a, Ill. Monogr. Ferula: 70, p. p.

Type: KAZAKHSTAN. "Mont. Dshungarienses, Tschartombaj, 16 VI 1909, Roshevitz 81" (lectotype LE!; designated by Pimenov, 2017. in Turczaninowia 20, 2: 142).

= Ferula angustiloba Pimenov, Bot. Zhurn. (Leningrad) 61(9): 1230. 1976.

Type: KYRGYZSTAN. "Tian-Schan septentrionalis, Kyrgyzstan, jugum Kungei-Alatau, declivitas australis prope trajectus Santach, ad marginem piceeti, 17 VII 1970, Pimenov \& Trusov 751" (holotype LE! [LE00065838, LE00065839]; isotype MW! [MW0565867]).

$=$ Ferula akitschkensis B. Fedtsch. ex KosoPol. var. kuldshensis Korovin, 1947a, Ill. Monogr. Ferula: 70.

Type: CHINA. "Kuldsha".

- Ferula karataviensis auct. non Korovin: Shen Kuanmien, 1992, in Shan Renhwa et Sheh Menglan (eds.), Fl. Reipubl. Popularis Sin. 55(3): 114.

- Ferula kirialovii auct. non Pimenov: Shen Kuanmien, 1992, in Shan Renhwa \& Sheh Menglan (eds.), Fl. Reipubl. Popularis Sin. 55, 3: 106, tab. 45 , fig. $1-3$.

- Ferula sumbul auct. non Hook.f.: Shen Kuanmien, 1992, in Shan Renhwa \& Sheh Menglan (eds.), Fl. Reipubl. Popularis Sin. 55, 3: 105.

Distribution in MA \& K: Kazakhstan (SE: Almaty prov.), Kyrgyzstan (Ysyk-köl prov., Talas prov., Chüh prov.).

Distribution in other Asian countries: China

Distribution area: Central and South-Western Asia.

Ferula alaica Pimenov et S. Melibaev, 1988, Bot. Zhurn. (Leningrad) 73(11): 1604, fig. 1, 2.

Type: KYRGYZSTAN. "Kyrgyzstan, jugum Alaicum, declivum septentrionale, montes Arpalyk, ad Juniperetorum collucatorum margines, 12 VII 1987, Pimenov et al. 265" (holotype LE! [LE01065834, LE01065835]; isotypes LE!, MW! [MW0593662, MW0593963]).

Distribution in MA \& K: Kyrgyzstan (Batken prov., Osh prov.). Endemic.

Distribution area: South-Western Asia.

Ferula angreni Korovin [1924, Byull. SredneAsiatsk. Gosud. Univ. 7: 20, in obs.], 1947a, Ill. Monogr. Ferula: 61, tab. 36, fig. 2. 
Type: UZBEKISTAN. "Ripa sinistra fl. Angren, inter Schaugasa et Kara-Mazar, regio Inula grandis, 25 VII 1924, Korovin 465-473" (lectotype TASH! [TASH002371-TASH002378]; designated as "typus". by Korovin (1947a: 61)).

Distribution in MA \& K: Kazakhstan S: S Kazakhstan prov.), Kyrgyzstan (Jalal-Abad prov., Naryn prov.), Tajikistan (Sughd prov.), Uzbekistan (Dzhizak prov., Toshkent prov.). Endemic.

Distribution area: South-Western Asia.

Ferula botschantzevii Korovin, 1964, Novit. Syst. Pl. Vasc. [1] 1964: 246.

Type: TAJIKISTAN. “Asia Media, Tajikistania australis, montes Koiki-tau, ad occidentem ab pag. Khoshady. 19 V 1960. Botschantzev \& Egorova 144" (holotype LE! [LE01065844], isotypes LE [LE01065845-LE01065849]).

= Ferula pachycaulos Rech. f., 1987, in Fl. Iranica (Rechinger) 162: 398, tab. 326, 327.

Type: AFGHANISTAN. "Afghanistan, NE, Kataghan: In jugo Paigah Kotal (Robotak, Mirza Atbili) inter Haibak et Pul-i Khumri, 1350 m., in declivibus argillosis, 10 VI 1962, Rechinger 16490" (holotype W! [W 1972-0013563]; isotypes E!, G! [G00366752]).

Distribution in MA \& K: Tajikistan (Khatlon prov., Dushanbe prov.).

Distribution in other Asian countries: Afghanistan.

Distribution area: Central and South-Western Asia.

Ferula canescens (Ledeb.) Ledeb., 1844, Fl. Ross. (Ledebour) 2: 302.

$\equiv$ Peucedanum canescens Ledeb. 1829, Fl. Altaic. (Ledebour) 1: 307.

Type: KAZAKHSTAN. "In subsalsis deserti soongoro-kirghisici inter Irtysch et montes Dolenkara nec non in regione occidentali, C. A. Meyer 239" (lectotype LE [LE00052139!], designated by Pimenov (2017: 143); isolectotypes CGE!, LE [LE00052140], P [P02272141-P02272143]).

Distribution in MA \& K: Kazakhstan (W: W Kazakhstan prov., Atyrau prov., Aktobe prov.; N: Kostonai prov., Pavlodar prov.; NE: E Kazakhstan prov.; Central: Karagangy prov., Kzyl-Orda prov.; S: Zhambyl prov.), Uzbekistan (Kara-kalpak).

Distribution in other Asian countries: China.

Distribution area: Central and South-Western Asia.

Ferula caspica M. Bieb. 1808, Fl. Taur.-Cauc. 1: 220 . $\equiv$ Peucedanum caspicum (M. Bieb.) Link, 1821, Enum. Hort. Berol. Alt. 1: 272.

Type: RUSSIA. "In deserto caucasico-caspica, ad viam publicam inter Astrachan et Kisljar [Ex planitiebus oppida Kisljar vicinis], Marchall von Bieberstein" (lectotype LE-Bieb!; designated by Yu. L. Menitski, 2008: 99; isolectotype MW - Hb. Trinius [MW0593978]).

= Ferula pumila Pall. ex Spreng., 1820, in Syst. Veg. ed. 15 bis (Roemer \& Schultes) 6: 598.

Type: RUSSIA. "Ad Wolgam, Pallas" (lectotype B-Willd!; designated here; isolectotype HAL [HAL0033090]). cf. Ferula wolgensis.

= Peucedanum gracile Ledeb., 1829, Fl. Altaic. (Ledebour) 1: 308.

$=$ Ferula gracilis (Ledeb.) Ledeb., 1844, Fl. Ross. (Ledebour) 2: 304.

Type: KAZAKHSTAN. "[Altai], in apricis, siccis, subsalsis deserti soongoro-kirghisici inter Buchtarminsk et lacum, qui Noor-Saissan vocatur. [Inter Buchtarminsk et lacum, qui NoorSaisan vocatur], 1826, Meyer 238" (lectotype LE! [LE00052102], designated by V. M. Vinogradova (2001: 54); isolectotypes HAL [HAL0098507], LE [LE00052103-LE00052106, LE00052108, LE00052109], P! [P02272152, P02272153], PH [PH00023034]).

= Peucedanum gracile Ledeb. var. microcarpum Ledeb., 1829, Fl. Altaic. (Ledebour) 1: 309.

Type: KAZAKHSTAN. "Altai, in lapidosis ad fl. Grammatucha prope Riddersk, Ledebour" (lectotype LE [LE00052107]; isolectotype CGE!)

= Peucedanum dubium Ledeb., 1829, Fl. Altaic. (Ledebour) 1: 310.

Type: RUSSIA. "Altai, in apricis siccis sterilissimis deserti editi ad fl. Tschuja mediis, 1826, Bunge [237]" (holotype LE!; designated by Pimenov, 2017, in Turczaninowia 20, 2: 142); isolectotypes C [C1008434], CGE!, LE!, P! [P02272153, P02272154, P02272155]).

$=$ Ferula wolgensis Willd. ex Ledeb., 1844, Fl. Ross. (Ledebour) 2: 203.

Type: RUSSIA. "Ad Wolgam" (syntypes B-Willd! [B-W 05773 01-06]) cf. Ferula pumila

Distribution in MA \& K: Kazakhstan (W: W Kazakhstan prov., Atyrau prov., Aktobe prov., Mangystau prov.; N: Kostonai prov., N Kazakhstan prov., Akmola prov., Pavlodar prov.; NE: E Kazakhstan prov.; Central: Karagandy prov., KzylOrda prov.; SE: Almaty prov.).

Distribution in other Asian countries: Russia, China, Mongolia, Turkey.

Distribution area: North, Central \& SouthWestern Asia. 
Ferula ceratophylla Regel et Schmalh., 1878, Trudy Imp. S.-Peterburgsk. Bot. Sada 5(2): 595.

Type: KAZAKHSTAN. "In Turkestaniae montibus Karatau prope $\mathrm{K}$ ultschek et ad montem HaraTschokla prope Bala-kschiata, 5000', 1876, A. Regel 242" (lectotype LE! [LE01065879], designated by V. M. Vinogradova 2000: 90; isolectotypes G! [G00366723], LE [LE01065880, LE01065883], P [P022721137]).

Distribution in MA \& K: Kazakhstan (S: S Kazakhstan prov., Zhambyl prov.), Uzbekistan (Toshkent prov.). Endemic.

Distribution area: South-Western Asia.

Ferula clematidifolia Koso-Pol., 1921, Bot. Mater. Gerb. Glavn. Bot. Sada R.S.F.S.R. 2(1): 64.

Type: UZBEKISTAN. "Fluvio Sangardak, declivum septentrionale montis Bel-Auty, circa vill. Bachscha. 23 V 1913. Michelson 1798" (lectotype LE! [LE01065856], designated by V. M. Vinogradova 2000: 90; isolectotype LE [LE01065857]; "Gazy-mailik Mts, Chosch-bulak. 08 V 1883. A Regel" (syntypes LE [LE01065858LE01065867].

Distribution in MA \& K: Tajikistan (Sughd prov., Khatlon prov., Dushanbe prov.), Uzbekistan (Qashqadarya prov., Samarqand prov., Surxondarya prov.). Endemic.

Distribution area: South-Western Asia.

Ferula conocaula Korovin, 1947a, Ill. Monogr. Ferula:33, tab. 8, fig. 1.

Type: TAJIKISTAN. "Mogol-tau: TschaschmaArzanak - Muzbek. 16 V 1924. Popov \& Vvedensky 711, 712, 713, 714, 715,717, 718, 719, 720, 721, 722, 724,725, 726, 727, 729, 734, 735" (holotype TASH! [TASH002385-TASH002399, TASH003697TASH003699]; isotypes LE! [LE01065876], MHA!).

Distribution in MA \& K: Tajikistan (Sughd prov.). Endemic.

Distribution area: South-Western Asia

Note: - It is very difficult to determine whether the numerous herbariums sheets of the species in type collection TASH (usually part of stem and leaf or part of stem and umbel) are parts of a single plant or several plants of the same population (most likely). If collectors used several plants, some sheets should be considered as isolectotypes, but which one is a big problem. See also F. tuberifera, Dorema sabulosum and some big Umbellifers.

Ferula czatkalensis Pimenov [1983, in Vvedensky (ed.), Opred. Rast. Sred. Azii 7: 376, 297, nom. inval. (Art. 40.2)]; 2000, Bot. Zhurn. (St. Perersburg) 85(1): 90 .

Type: KYRGYZSTAN. "Kyrgyzstan, jugum Czatkalense, declivum australe, angustis fl. Aflatun, ripa sinistra, ad declivibus lapidosis et saxis, 28 VIII 1975, Pimenov 1447" (holotype MW! [MW 0593986 - MW0593990], designated by Pimenov (Vinogradova 2000:90); isotypes LE! [LE01065876], MW! [MW0593991, MW0593992]).

Distribution in MA \& K: Kyrgyzstan (JalalAbad prov.). Endemic.

Distribution area: South-Western Asia.

Ferula czatkalensis Pimenov f. latisecta Pimenov, 1983, in Vvedensky (ed.), Opred. Rast. Sred. Azii 7: 376.

Type: KYRGYZSTAN. "Kyrgyzstan, prov. Osch, prope Taschkumyr, declivia lapidosa et schistosa, 09 VII 1976, Rakhmakulov \& Melibaev 1465" (holotype TASH! [TASH002400-TASH002403]).

Distribution in MA \& K: Kyrgyzstan (as the species).

Ferula decurrens Korovin, 1964, Novit. Syst. Pl. Vasc. [1] 1964: 247.

Type: TAJIKISTAN. "Asia Media, Tajikistania australis, prope pag. Bog ad fl. Piandsh, in valle riv. Schpilau, 01 VI 1960, Botschantzev \& Egorova 772" (lectotype LE! [LE01065886], isolectotypes [LE01065884, LE01065887]).

Distribution in MA \& K: Tajikistan (Khatlon prov.). Endemic.

Distribution area: South-Western Asia.

Ferula dissecta (Ledeb.) Ledeb., 1844, Fl. Ross. (Ledebour) 2: 301.

$\equiv$ Peucedanum dissectum Ledeb., 1829, Fl. Altaic. (Ledebour) 1: 306.

Type: KAZAKHSTAN. "In collibus ad rivulum Talowka prope Buchtarminsk, 17 VI 1826, Meyer 239" (lectotype LE! [LE00052110]; designated by Pimenov, 2017, in Turczaninowia 20, 2: 143); isolectotypes LE [LE00052111-LE00052119], P! [P02272139, P02272140]).

= Peucedanum ledebourii Steud., 1841, Nomencl. Bot. (Steudel), ed. 2, 2: 311, nom. illeg., non G. Don (1834).

$=$ Ferula rigidula DC. var. songarica Schrenk ex Fisch. et C. A. Mey., 1842, in Schrenk, Enum. Pl. Nov. 2: 43.

Type: KAZAKHSTAN. "In collibus prope Ajagus, Schrenk" (lectotype LE!; designated by Pimenov, 2017, in Turczaninowia 20, 2: 143); isolectotypes W! [W 1889-0049900], WU-Keck). 
Distribution in MA \& K: Kazakhstan (NE: E Kazakhstan prov.; S: Zhambyl prov.; SE: Almaty prov.).

Distribution in other Asian countries: China.

Distribution area: South-Western Asia.

Ferula diversivittata Regel et Schmalh., 1878, Trudy Imp. S.-Peterburgsk. Bot. Sada 5(2): 593, in adn.

Type: KAZAKHSTAN. "Prope stationem Tashsuam, in angustiis prope Dscham et Dschismak [?]. $20 \mathrm{~V}$ 1870. O. A. Fedtschenko" (lectotype LE! [LE01065889], designated by Pimenov \& Baranova 1979: 90); isolectotypes G! [G00366821], K! [K001097224], LE [LE1065888]).

$=$ Ferula suaveolens Aitch. et Hemsl., 1888, Trans. Linn. Soc. London, Bot. 3(1): 69, tab. 20, 21.

Type: IRAN. "Khorassan, in collibus S Bezg, $6000 \mathrm{ft}$., in shady places and in the vicinity of running water, 18 VI 1865, Aitchison [1064]" (lectotype K! [K001097221，K001097222, K001097223], designated here by Pimenov; isolectotypes G! [G00366759], GH [GH00076397]).

$=$ Ferula sintenisii H. Wolff, 1924, Repert. Spec. Nov. Regni Veg. 19: 311.

Type: TURKMENISTAN. "Regio Transcaspica; Aschabad, in montibus supra pagum Nephton. 04 V 1900. Sintenis 265a" (lectotype LE!, designated by V. M. Vinogradova 2000: 94); isolectotypes G! [G00366726, G00366753], GH, K! [K001097275, K001097276, K001097277], US [US00517437], $\mathrm{W}$ !).

Distribution in MA \& K: Kazakhstan (S: S Kazakhstan prov., Zhambyl prov.), Kyrgyzstan (Talas prov.), Uzbekistan (Navoi prov., Dzhizak prov., Samarqand prov., Qashqadarya prov., Surxondarya prov., Toshkent prov.), Turkmenistan (Balkan prov., Akhal prov., Lebap prov.).

Distribution in other Asian countries: Afghanistan, Iran.

Distribution area: South-Western Asia.

Ferula dshaudshamyr Korovin, 1947a, Ill. Monogr. Ferula: 79, tab. 16, fig. 1.

$\equiv$ Dorema songoricum Kar. et Kir., 1842, Bull. Soc. Imp. Naturalistes Moscou 15: 365, non Ferula soongarica Pall. ex Spreng.

$\equiv$ Ferula dubjanskyi Korovin ex Pavlov [1935, Byull. Sredne-Asiatsk. Gosud. Univ. 20: 196, nomen]; 1935, Fl. Centr. Kazakh. 2: 534, 539, nom. illeg. (Art. 39.1).

Type: KAZAKHSTAN. "In collibus sabulosis Songoriae inter fontem Sassyk-pastau et montes
Arganaty, 1841, Karelin \& Kirilov 1542" (lectotype LE!, designated by.V. M. Vinogradova 1999: 86; isolectotype $\mathrm{P}$ [P00835036]).

Distribution in MA \& K: Kazakhstan (W: Atyrau prov., Aktobe prov., Mangystau prov.; NE: E Kazakhstan prov.; Central: Karagandy prov., KzylOrda prov.; S: S Kazakhstan prov., Zhambyl prov.; SE: Almaty prov.), Uzbekistan (Kara-kalpak).

Distribution in other Asian countries: China.

Distribution area: Central and South-Western Asia.

Ferula dshizakensis Korovin, 1947a, Ill. Monogr. Ferula: 58, tab. 34, fig. 1.

Type: UZBEKISTAN. "Regio Seravschan, prope pag. Karnap, montes versus Mat-bulak. 29 V 1926. Popov 3/44" (holotype TASH! [TASH002404]).

Distribution in MA \& K: Tajikistan (Sughd prov.), Uzbekistan (Navoi prov., Dzhizak prov., Samarqand prov., Qashqadarya prov.). Endemic.

Distribution area: South-Western Asia.

Ferula equisetacea Koso-Pol., 1921, Bot. Mater. Gerb. Glavn. Bot Sada R.S.F.S.R. 2(1): 65.

Type: TAJIKISTAN. "In Buchara, Hissar, angust. Karatag, supra Chakkimi. 6000'. 24 V 1897. Lipsky (lectotype LE! [LE01065908], designated by V. M. Vinogradova 1997a: 100).

Distribution in MA \& K: Tajikistan (Khatlon prov., Dushanbe prov.). Endemic.

Distribution area: South-Western Asia.

Ferula eugenii Kamelin, 1971, Novit. Syst. P1. Vasc. 7: 268.

Type: TAJIKISTAN. "Tadshikistania, jugum Hissaricum, systema fl. Warzob, in valle fl.Kondara, in parte inferior. $20 \mathrm{~V}$ 1965. Kamelin 1-3" (holotype LE [LE01065906], isotype LE! [LE01065907]).

Distribution in MA \& K: Tajikistan (Dushanbe prov.). Endemic.

Distribution area: South-Western Asia.

Ferula fedoroviorum Pimenov, 1976, Bot. Zhurn. (Leningrad) 61(9): 1230, nom. inval. (Art. 40.2); 2000, Bot. Zhurn. (St. Perersburg) 81(1): 91.

Type: KYRGYZSTAN. "Asia Media, TianSchan centralis, vallis fl. Kokomeren, supra pag. Ksyl-oi ad praerupta lapidosa. 24 V 1970. Pimenov \& Borjaev 819" (holotype LE! [LE00065919]); isotypes LE [LE00065917, LE00065918]); MW! [MW0593982-MW0593985]); ibid., 30 VI 1971, Pimenov 1036" (paratypes LE!, MW! [MW0593979-MW0593981]). 
Distribution in MA \& K: Kyrgyzstan (JalalAbad prov., Naryn prov.). Endemic.

Distribution area: South-Western Asia.

Ferula fedtschenkoana Koso-Pol., 1921, Bot. Mater. Gerb. Glavn. Bot Sada R.S.F.S.R. 2(1): 66.

Type: TAJIKISTAN. "In reg. Samarkand, ArczaMajdan, in sylva, 08 VII 1913, B. A. Fedtschenko 92" (lectotype LE! [LE00065919], designated by V. M. Vinogradova 1997a: 100).

Distribution in MA \& K: Tajikistan (Sughd prov.), Uzbekistan (Dzhizak prov., Qashqadarya prov.). Endemic.

Distribution area: Central and South-Western Asia.

Ferula ferganensis Lipsky ex Korovin, 1947a, Ill. Monogr. Ferula: 59, tab. 35, fig. 2.

Type: KYRGYZSTAN. "Fergana, declivia septentrionalis montibus Alasch-tau, leviter infra terminis nivis, prope Rosae, 15 VI 1916, Rosanov 297' (holotype TASH! [TASH002405]).

Distribution in MA \& K: Kyrgyzstan (JalalAbad prov.). Endemic.

Distribution area: South-Western Asia.

Ferula foetida (Bunge) Regel, 1878, Trudy Imp. S.-Peterburgsk. Bot. Sada 5(2): 592, in nota.

三Scorodosma foetida Bunge, 1846, in Delect. Sem. Horti Dorpat.: unpaged [3].

Type: UZBEKISTAN. "In deserto aralensi specimen unicum florens, et pauca semina collegit $b$. Lehmann [Im der Lehmsteppe zwischen TschakyrAta und Nasarbai-Kuduk], 12 IV 1842, Lehmann 549" (lectotype LE!; designated by Pimenov, 2016, in Bot. Zhurn. 101(10): 1232; isolectotype P [P02272144, P02272145]).

- Ferula assa-foetida auct. non L.: Boiss., 1872, Fl. Orient. (Boissier) 2: 994.

Distribution in MA \& K: Kazakhstan (W: Aktobe prov., Mangystau prov.; Central: KzylOrda prov.; S: S Kazakhstan prov., Zhambyl prov.), Kyrgyzstan (Batken prov., Jalal-Abad prov., Osh prov.), Tajikistan (Sughd prov. Khatlon prov.), Uzbekistan (Kara-kalpak, Buxoro prov., Dzhizak prov., Qashqadarya prov., Navoi prov., Namangan prov., Samarqand prov., Surxondarya prov., Sirdarya prov., Toshkent prov., Khorezm prov.), Turkmenistan (Balkan vel, Deşoguz prov., Lebap prov., Mary prov.).

Distribution in other Asian countries: Pakistan, Afghanistan.

Distribution area: Central and South-Western Asia.
Ferula foetidissima Regel et Schmalh., 1878, Gartenflora 27: 195, tab. 944; 1878, Trudy Imp. S.-Peterburgsk. Bot. Sada 5(2): 593.

Type: TAJIKISTAN. "In valle fluvii Sarawschon prope Obburdon et Pachud, 5000' alt., 06 VI 1870, O. A. Fedtschenko" (lectotype LE! [LE01065924], designated by Pimenov et Baranova 1979: 86; isolectotype LE [LE01065923]).

- Ferula conocaula auct. non Korovin: Shen Kuanmien, 1975, Acta Phytotax. Sin. 13(3): 91.

Distribution in MA \& K: Kyrgyzstan (Batken prov., Osh prov.), Tajikistan (Sughd prov.), Uzbekistan (Dzhizak prov.).

Distribution in other Asian countries: China.

Distribution area: South-Western Asia.

Ferula galbaniflua Boiss. et Buhse, 1860, Nouv. Mém. Soc. Imp. Naturalistes Moscou 12: 99.

$\equiv$ Peucedanum galbanifluum (Boiss. et Buhse) Baill., 1879, Hist. P1. (Baillon) 7: 185.

Type: IRAN. "Am Fuss und an den Abhangen des Demavend, 4-8000 Fusshoch; auch im den Bergen bei Kuschak und Churchura, VI 1847 (1848?), Buhse 1137" (holotype LE! [LE00015770]; isotypes LE [LE00015772, LE00015773, LE00015776]).

- Ferula gummosa auct. non Boiss.: Korovin, 1950, in Nikitin (ed.), Fl. Turkmen. 5: 242.

Distribution in MA \& K: Turkmenistan (Balkan prov., Akhal prov.).

Distribution in other Asian countries: Iran.

Distribution area: South-Western Asia.

Ferula gigantea B. Fedtsch., 1902, Trudy Bot. Muz. Imp. Akad. Nauk 1: 135.

Type: TAJIKISTAN. "In valle fl. Pjandzh, in declivibus lapidosis inter pag. Darmarak et pag. Anderob, 8000', 25-27 VII 1901, B. A. Fedtschenko" (lectotype LE! [LE00050246-LE00050249], designated here by Pimenov; isolectotype B!, photo E! [E00433775]).

= Ferula inflata Korovin, 1947a, Ill. Monogr. Ferula: 35, tab. 10, fig. 2.

Type: TAJIKISTAN. Asia Media: Pamir-Alaj, montes Tadshikorum in regione Schuroabad; in regione fruticeta alt. $1840 \mathrm{~m}, 29$ VII 1935, Linczevski \& Maslennikova 852 (holotype LE! [LE00050263]).

= Ferula latifolia Korovin, 1947a, Ill. Monogr. Ferula: 34, tab.10, fig. 1

Type: TAJIKISTAN. "Asia Media: Karategin prope Kamtschirak, 25 VII 1916, B. A. Fedtschenko, An. P. Fedtschenko \& Knorring 1293" (lectotype LE! [LE00051828-LE00051830], designated here; isolectotype LE [LE00051831]). 
Distribution in MA \& K: Tajikistan (Kŭhistani Badakhshan, Khatlon prov., Dushanbe prov.), Uzbekistan (Surxondarya prov.).

Distribution in other Asian countries: Afghanistan.

Distribution area: South-Western Asia.

Ferula glaberrima Korovin, 1947, Ill. Monogr.: 26, tab. 3, fig. 2.

Type: KAZAKHSTAN. "Asia Media, arenae Mujun-kum, 14 VI 1922, Drobov 520" (holotype TASH! [TASH002406]; isotype TASH [TASH002407]).

Distribution in MA \& K: Kazakhstan (Central: Kzyl-Orda prov.; S: Zhambyl prov.). Endemic.

Distribution area: Central Asia.

Ferula grigoriewii B. Fedtsch., 1902, Trudy Bot. Muz. Imp. Akad. Nauk 1: 137.

Type: TAJIKISTAN. "Plantae Turkestanicae. Schugnan, in valle fl. Gunt, Vankala-Rivak, 22 VII 1901, O. A. et B. A. Fedtschenko" (lectotype LE! [LE00050236], designated by V. M. Vinogradova 2000: 91).

Distribution in MA \& K: Tajikistan (Kŭhistani Badakhshan).

Distribution in other Asian countries: Afghanistan

Distribution area: South-Western Asia.

Ferula gummosa Boiss., 1856, Diagn. P1. Orient. (ser. 2) 2: 92.

$\equiv$ Ferula galbaniflua Boiss. et Buhse var. aucheri Boiss., 1872, Fl. Orient. (Boissier) 2: 989.

Type: IRAN. "In Persia, Aucher-Eloy 3658" (lectotype P!; designated here by Pimenov; isolectotypes G! [G00366734], K! [K000568182], MPU [MPU020984]).

= Ferula badrakema Koso-Pol., 1921, Bot. Mater. Gerb. Glavn. Bot Sada R.S.F.S.R. 2(1): 62.

Type: TURKMENISTAN. "Kuschka, in arenosis, 06 IV 1905, Androssov" (lectotype LE!, designated by V. M. Vinogradova 1997a: 100).

$=$ Ferula afghanistanica M. Hiroe, 1958, Umbellif. Asia 1: 185.

Type: AFGHANISTAN. "Mak. El. 8500', 03 X 1939, Koelz 14071" (holotype UC! [UC651046]).

- Ferula galbaniflua auct. non Boiss. et Buhse: Aitch. 1888, Trans. Linn. Soc. Bot. London, (2 ser.) 3: 68 .

Distribution in MA \& K: Turkmenistan (Mary prov.).
Distribution in other Asian countries: Afghanistan, Iran.

Distribution area: South-Western Asia.

Ferula gypsacea Korovin, 1947, I1l. Monogr. Ferula: 65, tab. 23, fig. 1.

Type: KAZAKHSTAN. "Tian-Schan occidentalis, mont. Alym-Tau, 18 VI 1925, Kultiassov 137" (holotype TASH! [TASH002408]; isotypes TASH [TASH002409, TASH002410]).

Distribution in MA \& K: Kazakhstan (S: S Kazakhstan prov.). Endemic.

Distribution area: South-Western Asia.

Ferula helenae U. Rakhmankulov et S. Melibaev, 1982, Novit. Syst. Pl. Vasc. 19: 117.

Type: UZBEKISTAN. "Uzbek SSR, Nuratau, montes Pistali-tau, prope pag. Balykchi, in declivis schistosis. 18 IV 1977. Rakhmankulov et Melibaev 1483" (holotype LE?, isotype TASH!]; paratypes (12 IV 1975, Rakhmankulov et Melibaev 1289) TASH [[TASH002413, TASH002415-TASH002417]).

Distribution in MA \& K: Uzbekistan (Dzhizak prov.). Endemic.

Distribution area: South-Western Asia.

Note: Both Rakhmankulov \& Melibaev (1982) and Vinogradova (1997a) reported that the holotype is in LE which we unfortunately failed to locate. We believe it is actually in TASH.

Ferula hissarica Pimenov et Kljuykov, 2014, Bot. Zhurn. (St. Petersburg) 99(5): 595.

Type: TAJIKISTAN. "Tajikistania, jugum Karateginum, systema fluminis Obi-Komarou, prope ostium fluminis Darai-Tonchak, in glareosis, inter lapides amplas, $\mathrm{h}=1544 \mathrm{~m}, \mathrm{~N} 39^{\circ} 09.64^{\prime}$, E70 21.33'. 03 VIII 2012. Pimenov, Kljuykov \& Ukrainskaja 7-12" (holotype MW! [MW0595550]).

Distribution in MA\& K: Tadjikistan (Dushanbe prov.). Endemic.

Distribution area: South-Western Asia.

Ferula iliensis Krasn. ex Korovin,1947, Ill. Monogr. Ferula: 34.

Type: KAZAKHSTAN. "Asia Media, deserta Balhaschensis, monte Boguty et Soguty inter stat. Taldykuduk et Kisil-Kija, Krasnov" (holotype E; isotype $\mathrm{W} !)$.

$=$ Ferula popovii Korovin, 1962, Trudy Inst. Bot. Akad. nauk Kazakhsk. SSR 13: 258.

Type: KAZAKHSTAN. "Dshungarsky Alatau (pars meridionalis), mons Ulkunkalkan, ad pla- 
nitiem argillaceo-petraeum, 21 VI 1959, Sinicin" (lectolotype AA!, designated by V. P. Goloskokov, 1963: 30); "VI 1959, Sinicin" (syntype AA!); "In regione montis Bolschie Boguty, 26 VI 1958, Bykov \& Godvinski" (syntype AA!).

Distribution in MA \& K: Kazakhstan (SE: Almaty prov.).

Distribution in other Asian countries: China.

Distribution area: Central Asia.

Ferula inciso-serrata Pimenov et Yu. V. Baranova, 1979, Byull. Moskovsk. Obshch. Isp. Prir. Otd. Biol. 84(3): 87, fig. 1.

Type: KYRGYZSTAN. "Kyrgyzstan australis, jugum Czatkalense, in valle fluvii Kassansai, inter Kysyl-Tokoi et Terek-sai, montes gypsacei et rubroarenosi, 14 VI 1971, Pimenov, Baranova \& Kljuykov" (holotype MW! [MW0594005]; isotypes LE!, MW [MW0594004]).

- Ferula foetidissima auct. non Regel et Schmalh.: Golovkova, 1959, in Fl. Kirgh. SSR 8: 86, p. p.

Distribution in MA \& K: Kyrgyzstan (JalalAbad prov.). Endemic.

Distribution area: South-Western Asia.

Ferula juniperina Korovin, 1959, Bot. Mater. Gerb. Bot. Inst. Komarova Akad. Nauk SSSR 19: 338. Type: UZBEKISTAN. "Montes Thian-Schan occidentalis, pars superior fluv. Angren, in valle Urges-say, inter silvas juniperinas, 21 VI 1953, E. P. Korovin \& S. E. Korovin 450-453" (holotype TASH! [TASH002424-TASH002427]).

$=$ Ferula kirialovii Pimenov, 1979, Byull. Moskovsk. Obshch. Isp. Prir. Otd. Biol. 84(5): 110.

Type: UZBEKISTAN. "Tian-Schan occidentalis, Tschimgan, 16 VII 1897, O. A. Fedtschenko" (holotype LE! [LE00051814, LE00051815, LE00051816]).

- Peucedanum pseudoreoselinum auct. non Regel et Schmalh.: O. et B. Fedtsch., 1909, Consp. fl. Turkest. (O. A. Fedtschenko et B. A. Fedtschenko) 3: 110 , p. p.

- Ferula pseudoreoselinum auct. non Koso-Pol.: Korovin, 1947, Ill. Monogr. Ferula: 64, tab. 15, fig. 2.

Distribution in MA et K: Kazakhstan (S: S Kazakhstan prov. Dzambyl prov.), Kyrgyzstan (Jalal-Abad prov.), Tajikistan (Sughd prov.), Uzbekistan (Toshkent prov.). Endemic.

Distribution area: South-Western Asia.

Note: The name of priority (F. juniperina) for the species was clarified after the publication of a critical revision (Pimenov, 1979) of sect. Euryangium of the genus Ferula, to which the species belongs. This was possible during a checking of the type collection in TASH. Type material of F. juniperina from basin of riv. Angren is not in good condition; so its identification required some additional efforts.

Ferula karakalensis Korovin, 1947, Ill. Monogr. Ferula: 29, tab. 5, fig. 2.

Type: TURKMENISTAN. "Regio Transcaspica, distr. Karakala, ad st. Dshalil. 13 V 1916. Czerniakowska 1024" (holotype LE! [LE00050264, LE00050265]).

$=$ Ferula tersakensis Korovin, 1947, Ill. Monogr. Ferula : 26, tab. 4, fig. 2.

Type: TURKMENISTAN. "Tersakan, Radde" (holotype LE [LE00051918]).

Distribution in MA \& K: Turkmenistan (Balkan prov.).

Distribution in other Asian countries: Iran.

Distribution area: South-Western Asia.

Ferula karatavica Regel et Schmalh., 1878, Trudy Imp. S.-Peterburgsk. Bot. Sada 5(2): 594.

Type: KAZAKHSTAN. "In Turkestaniae montibus karatavicis prope Boroldai. $03 \mathrm{~V} 1876$. A. Regel" (lectotype LE! [LE00050268], designated here; isolectotypes LE [LE00050266, LE00051783, LE00051784, LE00051785])

$=$ Ferula sassyr Koso-Pol., 1916, Izv. Imp. S.-Peterburgsk. Bot. Sada 16: 224.

Type: UZBEKISTAN. "Inter Syr-Darja et Taschkent. 700-1400'. 20 V 1871. O. A. Fedtschen$k o$ " (holotype LE! [LE00051888]; isotype MW?).

= Ferula aitchisonii Koso-Pol., 1921, Bot. Mater. Gerb. Glavn. Bot Sada R.S.F.S.R. 2(1): 61.

Type: KYRGYZSTAN. "In prov. Fergana, distr. Andishan, in declivibus mont. Kulagan-tau, 08 VII 1911, Knorring \& Minkwitz 1452" (holotype LE! [LE01065822, LE01065823]).

= Ferula eremophila Korovin, 1947, Ill. Monogr. Ferula: 68.

Type: KAZAKHSTAN. "Asia Media, Kazakh SSR, in vicunitate laci Bijlukul, in vallis siccis elevates, 15 VIII 1934, Ponomareva" (lectotype AA! (Goloskokov, 1963; isolectotype TASH! [TASH003713] - only fruits).

Distribution in MA \& K: Kazakhstan (S: S Kazakhstan prov., Zhambyl prov.), Kyrgyzstan (Jalal-Abad prov., Osh prov.), Tajikistan (Sughd prov.), Uzbekistan (Toshkent prov.). Endemic.

Distribution area: South-Western Asia. 
Ferula karataviensis (Regel et Schmalh.) Korovin [1933, ex Pavlov, 1933, Byull. Moskivsk. Občš. Isp. Otd. Biol. 42: 130, nomen];], 1947, Ill. Monogr. Ferula: 72, tab. 15, fig. 1.

$\equiv$ Peucedanum karataviense Regel et Schmalh., 1878, Trudy 1978, Imp. S.-Peterburgsk. Bot. Sada 5(2): 592 .

$\equiv$ Ferula korovinii Pavlov, 1933, Byull. Moskovsk. Obshch. Isp. Prir. Otd. Biol. 42: 129, nom. illeg. (Art. 51.1, 52.1, 53.3, Ex. 10).

Type: KAZAKHSTAN. "In Turkestaniae montibus karatavicis prope Ischtube [Karatau, AtschTube], 03 VI 1876, A. Regel 456" (lectotype LE! [LE00052130], designated by Korovin 1951: 138; isolectotype LE [LE00052131, LE00052132]).

Distribution in MA \& K: Kazakhstan (Central: Kzyl-Orda prov.; S: S Kazakhstan prov., Zhambyl prov.), Kyrgyzstan ((Talas prov., Chüh prov.). Endemic.

Distribution area: Central and South-Western Asia.

Ferula karategina Lipsky ex Korovin, 1926, Byull. Sredne-Asiatsk. Gosud. Univ. 14, Suppl. (Sched. Herb. Fl. As. Med. 10): 13.

Type: TAJIKISTAN. "Buchara, Karategin, Gorif, 7200', 06 VIII 1896, Lipsky" (lectotype LE! [LE00051785], designated by Korovin 1951: 108).

Distribution in MA \& K: Kyrgyzstan (Batken prov.), Tajikistan (Sughd prov., Dushanbe prov.), Uzbekistan (Surxondarya prov.). Endemic.

Distribution area: South-Western Asia.

Ferula karelinii Bunge, 1852, Beitr. Fl. Russ1. (Lehm. Rel. Bot.): 130.

$\equiv$ Schumannia karelinii (Bunge) Korovin, 1947, Ill. Monogr. Ferula: 81.

Type: UZBEKISTAN. "In der Lehmsalzsteppe Karakum, 28 VI 1841, Lehmann 544" (lectotype LE! [LE00051782], designated by V. M. Vinogradova 2002: 139; isolectotype LE [LE00051792LE00051796]); KAZAKHSTAN. "In alten Flussbette des Jan. 03 V 1842. Lehmann" (syntypes P [P02272161, P02272162]).

= Schumannia turcomanica Kuntze, 1887, Trudy Imp. S.-Peterburgsk. Bot. Sada 10: 192.

Type: TURKMENISTAN. "Zwischen KisilArwat und dem Kaspi-See. Kuntze (NY?)".

- Ferula peucedanifolia auct. non Willd.: Kar. et Kir. 1842, Bull. Soc. Imp. Naturalistes Moscou 15: 165.

Distribution in MA \& K: Kazakhstan (W: Aktobe prov., Mangystau prov.; N: Akmola prov.; Central: Karagandy prov, Kzyl-Orda prov.; S: S Ka- zakhstan prov., Zhambyl prov.; SE: Almaty prov.), Tajikistan (Sughd prov., Khatlon prov.), Uzbekistan (Kara-kalpak, Andijon prov., Buxoro prov., Navoi prov., Surxondarya prov., Fergona prov.), Turkmenistan (Balkan prov., Daşoguz prov., Akhal prov., Lebap prov., Mary prov.).

Distribution in other Asian countries: China, Pakistan, Afghanistan, Iran.

Distribution area: Central and South-Western Asia, East Europe (Kalmykia).

Ferula kelifi Korovin, 1947, Ill. Monogr. Ferula: 25, tab. 1(3), 2(1). [Herbarium Florae URSS N 6417].

Type: UZBEKISTAN. "Montes Sogdano-Transoxani, in gypsaceis prope $\mathrm{p}$. Taschkak (orientem versus urbis Bajsun). $09 \mathrm{~V} \mathrm{1930.} \mathrm{Botschantzev} \mathrm{\&} \mathrm{Vve-}$ densky 882(6417)"(lectotype TASH! [TASH002432, TASH003693-TASH003695], isolectotypes BM! [BM000944767], BP!, BR [BR0000005420124], C! [C10005758], ERE [ERE0005557], G! [G00366840], LE! [LE00051800-LE00051802], LW [LW214965], M! [M0173058], MAK, MHA!, MW! [MW0594006], P! [P02272166], S! [S1221678], SP [SP003564], TNS!, US [US00664201], VAL [VAL10104], W!, WIS [WISv0255968WIS]).

$=$ Ferula primaeva Korovin, 1947, Ill. Monogr. Ferula: 26.

Type: UZBEKISTAN. "Asia Media, prope urb. Bajssun, Burju-Tacht. 13 V 1930. Lepeschkin" (holotype TASH! [TASH002487]).

Distribution in MA \& K: Tajikistan (Khatlon prov., Dushanbe prov.), Uzbekistan (Qashqadarya prov., Surxondarya prov.), Turkmenistan (Lebap prov.). Endemic.

Distribution area: South-Western Asia.

Note: The exiccates of this species and Ferula tuberifera were prepared by A. I. Vvedensky in 1935-36 for their publication in the Herbarium Florae Asiae Mediae series; this edition was not carried out. The material on Ferula fully corresponds to "Illustrated monograph of the genus Ferula" by Korovin (1947), including typification. This data was only published in 1986 in the Schedae ad Herbarium Florae URSS (book 25).

Ferula kelleri Koso-Pol., 1922, Bot. Mater. Gerb. Glavn. Bot. Sada R.S.F.S.R. 3(18): 171.

Type: KAZAKHSTAN. "Regio Heptapotamica, a Kara-bulak ad trajectum Kastek, ad declivi, 12 VII 1908, B. A. Fedtschenko 1579" (holotype LE! [LE00051810 - LE00051812], isotypes LE! [LE00051807-LE00051809]). 
Ferula foliosa Lipsky ex Korovin, 1947, Ill. Monogr. Ferula: 43, tab. 18, fig. 1

Type: KYRGYZSTAN. "Uzgen [F1. Jassy prope Usgent, 5-6000']. 28-30 V 1880. A. Regel" (lectotype LE! [LE01065927, LE01065929]; designated here; isolectotypes LE [LE01065932LE01065941], LECB [LECB0002304]).

Distribution in MA \& K: Kazakhstan ( $\mathrm{S}$ : Zhambyl prov.; SE: Almaty prov.), Kyrgyzstan (Jalal-Abad prov., Ysyk-köl prov., Naryn prov., Osh prov., Chüh prov.). Endemic.

Distribution area: South-Western Asia.

Ferula kokanica Regel et Schmalh., 1878, Trudy Imp. S.-Peterburgsk. Bot. Sada 5(2): 593.

Type: KYRGYZSTAN. "In Kokaniae valle fluvii Isfairam, 8000 ft. 20 VII 1871. O. A. Fedtschenko" (holotype LE! [LE00051817, LE00051818]).

= Ferula schugnanica B. Fedtsch., 1902, Trudy Bot. Muz. Imp. Akad. Nauk 1: 136.

Type: TAJIKISTAN. "In valle fl. Gunt, in declivibus lapidosis ab loco Vankala ad pag. Rivak, 8-10000'. 22 VII 1901. B. A. Fedtschenko" (holotype LE! [LE00051891-LE00051896]).

Distribution in MA \& K: Kyrgyzstan (Batken prov., Osh prov.), Tajikistan (Kŭhistani Badakhshan, Sughd prov., Khatlon prov., Dushanbe prov.), Uzbekistan (Dzhizak prov., Qashqadarya prov., Samarqand prov.).

Distribution in other Asian countries: India, Pakistan, Afghanistan.

Distribution area: Eastern and South-Western Asia.

Ferula kopetdaghensis Korovin, 1947, Ill. Monogr. Ferula: 61, tab. 38, p. p., excl. pl. e PamiroAlai.

Type: TURKMENISTAN. "Regio Transcaspica, angustis Iol-dere. 22 VI 1917. Korovin" (holotype TASH! [TASH002433]).], designated here).

Distribution in MA \& K: Turkmenistan (Balkan prov., Akhal prov.).

Distribution in other Asian countries: Iran.

Distribution area: South-Western Asia.

Ferula korshinskyi Korovin, [1928, Byull. Sredne-Asiatsk. Gosud. Univ. 1947, Ill. Monogr. Ferula: 68, tab. 45, fig. 1.

Type: KYRGYZSTAN. "Fergana, Naryn, supra Ketmen-tjube. 02 VIII 1926. Korovin 788, 792, 793, 795" (holotype TASH! [TASH002434, TASH002435, TASH002436, TASH002437]).
Distribution in MA \& K: Kyrgyzstan (Batken prov., Jalal-Abad prov., Naryn prov., Osh prov.), Uzbekistan (Fergona prov.). Endemic.

Distribution area: South-Western Asia.

Ferula koso-poljanskyi Korovin, 1947, Ill. Monogr. Ferula: 46, tab. 22, fig. 2.

Type: TAJIKISTAN. "Buchara, Darvaz, ad fl. Pjandsh, 4000'. 11 VII 1899. Lipsky" (lectotype LE! [LE00051820, LE00051822-LE00051825]; designated by V. M. Vinogradova 2002b: 139; "ibid. Bukhara, Darwas, Viskharvi, 1407 1899. Lipsky" (syntype LE [LE00051826]; “ibid. Bukhara, along the river Voszhila, a tributary of Panj. 11 VII 1899. Lipsky" (syntype LE [LE00051821]; AFGHANISTAN. "Darwaz, Omar, ad fluvii Pändsh, ripa sinistra. 09. 1882. A. Regel" (syntype LE [LE00051819]).

Distribution in MA \& K: Tajikistan (Kŭhistani Badakhshan).

Distribution in other Asian countries: Afghanistan.

Distribution area: South-Western Asia.

Ferula krylovii Korovin, 1934, Sist. Zametki Mater. Gerb. Krylova Tomsk. Gosud. Univ. 2-3: 2.

Type: KAZAKHSTAN. "Prov. Heptapotamica, distr. Zaisan, prope pag. Buran, m. Aschutas, steppa deserta subsalsuginosa. 09 VII 1928. Krylov \& Sergievskaya" (lectotype TK!; designated as "type" by A. V. Polozhij \& V. F. Balashova (1989: 26); isolectotypes LE! [LE00051827], TK).

- Ferula teterrima auct. non Kar. et Kir.: Krylov, 1935, Fl. Zapadnoi Sibiri 8: 1995.

Distribution in MA \& K: Kazakhstan (NE: E Kazakhstan prov.).

Distribution in other Asian countries: China.

Distribution area: Central Asia.

Ferula kuhistanica Korovin, 1947, Ill. Monogr. Ferula: 36.

Type: UZBEKISTAN. "Asia Media, montes Zeravschanici, in valle fl. Aman-Kutan. V 1935. Kudrjaschov" (holotype TASH! - not found).

- Ferula jaeschkeana auct. non Vatke: O. et B. Fedtsch., 1909, Consp. Fl. Turkest. (O. A. Fedtschenko \& B. A. Fedtschenko) 3: 106, p. p.

Distribution in MA \& K: Kyrgyzstan (Batken prov., Jalal-Abad prov., Naryn prov., Osh prov., Chüh prov.), Tajikistan (Sughd prov., Khatlon prov., Dushanbe prov.), Uzbekistan (Dzhizak prov., Samarqand prov., Qashqadarya prov., Surxondarya prov.), Turkmenistan (Lebap prov.). 
Distribution in other Asian countries: Afghanistan.

Distribution area: South-Western Asia.

Ferula kyzylkumica Korovin, 1959, in Vvedensky (ed.), Fl. Uzbekist. 4: 490, 426.

Type: UZBEKISTAN. "Kysyl-kum, SW margo, prope scaturiginem Chal-ata, montes Chalata $\mathrm{E}$ ad scaturginem in fissuris rupium. $19 \mathrm{~V}$ 1937. Botschantzev 512" (holotype TASH [№oㅡ 169104, 169105]; isotypes LE, LECB, TASH! [TASH002438, TASH002439]); "Prope puteum Ajak-kudzhumdy, in gypsaceis fuscoarenosis. 14 V 1937. Botschantzev 437" (syntype TASH); "Prope scaturiginem Dzhangeldy, in gypsaceis SE a scaturiginis. In saxoso arenario fusco destructis. 17 VII 1937. Botschantzev 473" (syntype TASH); "Kyzykkum, prope pagi Tashy-kuduk. 12 VI 1931. Muravljansky 255" (syntype TASH); "Kyzylkum, prope putium Dzhilandy, in declivia australibus schistosis Altyntau. $16 \mathrm{~V}$ 1957. Adylov 262" (syntype TASH); "S Kyzylkum, 5 km S a Tamdy, in monticulis Bakpaktau. $\mathrm{h}=400-450 \mathrm{~m} .22 \mathrm{~V}$ 1956. Li 239" (syntype TASH); "4 km NE a puteum Aitym, e substratis lapidoso-sceleticis. 27 V 1956. Li 274" (syntype TASH).

- Ferula microloba auct. non Boiss.: Korovin, 1959, in Fl. Uzbekist. 4: 411, p. p., excl. syn. Ferula collina.

Distribution in MA \& K: Uzbekistan (Karakalpak, Buxoro prov., Navoi prov. Samarqand prov.). Endemic.

Distribution area: Central and South-Western Asia.

Ferula latisecta Rech. f. et Aellen, 1952, Anz. Oesterr. Akad. Wiss., Math.-Naturwiss. K1. 89(11): 173.

Type: IRAN. "Khorasan: Montes Hazar Masdjid, inter Ardak et Tolgor, ca. 1200-1600 m, in rupibus fissures. 07-10 VI 1948. Rechinger \& Aellen 4988" (holotype W! [W 1963-0004947, W 1963-0004950], W 1963-004951; isotypes B! [B 10 0367203], E! [E00002489], G! [G00366844], K! [K001097290], $\mathrm{M}$ ! [M0173057]).

= Ferula undulata Pimenov et Yu. V. Baranova, 1976, Byull. Mo skovsk. Obshch. Isp. Prir. Otd. Biol. 81(2): 143, tab. 1, 2.

Type: TURKMENISTAN. "Turcomania, montes Kopet-Dagh, pars centralis, inter Cheirabad et Tschaek. 26 V 1973. Pimenov \& Baranova 444" (holotype LE! [LE00051934, 1E00051935]; isotype MW! [MW0594035]).
Distribution in MA \& K: Turkmenistan (Akhal prov.).

Distribution in other Asian countries: Iran.

Distribution area: South-Western Asia.

Ferula lehmannii Boiss., 1872, Fl. Orient. (Boissier) 2: 992.

Type: KAZAKHSTAN. "In desertis argillosis salsis, in desertum Kisilkum Turkestaniae borealis. [In deserto Kisil-kum]. 16 V 1842. Lehmann 543" (lectotype LE! [LE00051834]; designated by V. M. Vinogradova 2000: 93); isolectotypes G-BOIS!, LE! [LE00051833, LE00051835], P! [P02272167], W! [W0023613]).

- Ferula persica auct. non Willd.: Bunge, 1852, Mem. Acad. Imp. Sci. St.-Petersbourg Divers Savans 7: 305 .

Distribution in MA \& K: Kazakhstan (W: Atyrau prov., Aktobe prov., Mangystau prov.; Central: Karagandy prov., Kzyl-Orda prov.; S: Zhambyl prov.), Uzbekistan (Kara-kalpak). Endemic.

Distribution area: Central and South-Western Asia.

Ferula leiophylla Korovin, 1947a, Ill. Monogr. Ferula: 44, tab. 20, fig. 2.

Type: KAZAKHSTAN. "Distr. Kapal, steppa arenosa secundum riv. Yanach. 09 VI 1915. Titov 41" (holotype TASH! [TASH002457]).

Distribution in MA \& K: Kazakhstan (NE: E Kazakhstan prov.; S: Zhambyl prov.; SE: Almaty prov.), Kyrgyzstan (Chüh prov.). Endemic.

Distribution area: Central Asia.

Ferula leucographa Korovin, 1947a, Ill. Monogr. Ferula: 64, tab. 41, fig. 1.

Type: KAZAKHSTAN. "Prov. Syr-Darja, distr. Chimkent, Kelte-Maschat, in declivibus conglomeratorum. 15 VI 1923. Mokeeva 291" (holotype TASH! [TASH002458, TASH002459]).

= Ferula involucrata Korovin, 1947a, Ill. Monogr. Ferula: 65, tab. 41, fig. 2.

Type: KAZAKHSTAN. "Kara-tau, in mont. Ak-Tash [ Prov. Syr-Darya, distr. Aulie-ata, Mt. S Karatau, riv. Koktal]. 01 VI 1925. Sovetkina 293" (holotype TASH! [TASH002421]; isotypes TASH! [TASH002422, TASH002423]).

Distribution in MA \& K: Kazakhstan (S: S Kazakhstan prov., Zhambyl prov.). Endemic.

Distribution area: South-Western Asia.

Ferula linczevskii Korovin, 1947a, Ill. Monogr. Ferula: 45, tab. 21, fig. 2. 
Type: TAJIKISTAN. "Asia Media, in mont. Tadshikorum Kugi-Frusch, prope pag. Oin-Garan, 03 IX 1935, Linczevski \& Maslennikova 1289" (holotype LE!" [LE00051836]; isotype TAD!).

Distribution in MA \& K: Tajikistan (Kŭhistani Badakhshan, Sughd prov., Khatlon prov., Dushanbe prov.). Endemic.

Distribution area: South-Western Asia.

Ferula lipskyi Korovin, 1947a, Ill. Monogr. Ferula: 46, tab. 23, fig. 2.

Type: KYRGYZSTAN. "Prov. Fergana, distr. Margelan, ad monticolis schistosis S pag. Myntjube. 07 V 1913. Desjatov 1740" (holotype LE! [LE00051837, LE00051838]).

Distribution in MA \& K: Kyrgyzstan (Batken prov., Jalal-Abad prov., Osh prov.), Tajikistan (Sughd prov.), Uzbekistan (Fergona prov.). Endemic.

Distribution area: South-Western Asia.

Ferula lithophila Pimenov, 1981, in S. K. Cherepanov, Sosud. Rast. SSSR: 21.

三 Peucedanum mogoltavicum Korovin, 1947, Not. Syst. Herb. Inst. Bot. \& Zool. Acad. Sci. Uzbekistan 8: 9, non Ferula mogoltavica Korovin.

Type: TAJIKISTAN. "Tian-Schan, montes Mogol-tau, in graniticis supra Tschasma-arzanak, 08 IX 1924, Popov 846" (holotype TASH! TASH002552, TASH002553]; isotype LE! [LE00052133]).

Distribution in MA \& K: Tajikistan (Sughd prov.), Uzbekistan (Namangan prov.). Endemic.

Distribution area: South-Western Asia.

Ferula litwinowiana Koso-Pol., 1922, Sched. Herb. Fl. Ross. 8: 111; 1924, Not. Syst. Herb. Horti Petrop. 5: 49.

Type: TURKMENISTAN. "Regio Transcaspica, distr. Merw [Mary] arenae prope stationem viae ferreae Repetek, 16 IV 1913, Androssov 2546, 2617" (lectotype LE! [LE00051839LE00051841], designated by V. M. Vinogradova 2000: 93); isolectotypes BP!, C [C10008437], DAO [DAO000455720], E, ERE [ERE000555, ERE0005556], GH [GH00076398], K [photo K001097278], LE [LE00051842], M! [M01730[6], MW! [MW0594007, MW0594008], NSK [NSK0000510, NSK0000511], TK, WIS [WISv025[5966WIS, WISv0255967WIS]).

= Merwia androssowii B. Fedtsch., 1924, Bot. Mater. Gerb. Glavn. Bot. Sada R.S.F.S.R. 5: 49.

$=$ Ferula androssowii (B. Fedtsch.) M. Hiroe, 1979, Umbelliferae World: 1522.
Type: TURKMENISTAN. "Turkmenia - Regio transcaspica, distr. Merw, prope stationem viae ferreae Repetek, 15(28) IV 1916, Androssov" (lectotype LE!, designated by V. M. Vinogradova (2001: 54); "Repetek, arenae grumosae. 26.04-19 V 1916. Dubiansky" (syntype); "Repetek, arenae fixae. 23 IV- 06 V 1916. Dubiansky" (syntype).

Distribution in MA \& K: Kazakhstan (S: S Kazakhstan prov.), Uzbekistan (Khorezm prov., Buxoro prov., Navoi prov., Qashqadarya prov.), Turkmenistan (Balkan prov., Daşoguz prov., Akhal prov., Lebap prov., Mary prov.). Endemic.

Distribution area: Central and South-Western Asia.

Ferula malacophylla Pimenov et Yu. V.Baranova, 1977, Byull. Glavn. Bot. Sada 106: 43.

Type: KAZAKHSTAN. "Kazachstania australis, prov. Dzhambul, in promontoriis orientalibus jugi Karatau, ad ripas lacus Aschtschilkul, in salsas. 02 V 1971. Pimenov, Pakaln \& Sdobnina 40" (holotype LE!; isotypes MW! [MW0594009-MW00594012]).

Distribution in MA \& K: Kazakhstan (Central: Kzyl-Orda prov.; S: Zhambyl prov.). Endemic.

Distribution area: South-Western Asia.

Ferula mogoltavica Lipsky ex Korovin, 1947, Ill. Monogr. Ferula: 28, tab. 7, fig. 2.

Type: TAJIKISTAN. "Montes Mogoltau, declivia australia lapidosa prope exitus viae ab trajectum Badambek ex montibus, 11IV 1927, Granitov 199, 200" (holotype TASH! [TASH002471]; isotype LE! [LE00051844]).

Distribution in MA \& K: Tajikistan (Sughd prov.). Endemic.

Distribution area: South-Western Asia.

Ferula narthex Boiss., 1872, Fl. Orient. (Boissier) 2: 994.

$\equiv$ Narthex asafoetida Falc. ex Lindl., 1846, Gard. Chron.: 743; 1846, Proc. Linn. Soc. London 1(30): 309; 1846, Trans. Linn. Soc. 20: 285, non Ferula assafoetida $\mathrm{L}$.

$\equiv$ Peucedanum narthex (Boiss.) Baill., 1879, Hist. Pl. (Baillon) 7: 185.

Type: INDIA. "[West Tibet] Inter saxa in valle Astore vel Hussorah dictae prope Indum, ultra Cashmeer 14.07 Boostbon [Hassere, Astore bvalley], 23 IX 1838, Falconer [Loftus] 495" (lectotype K! [K001097265]; designated here by Pimenov; isolectotypes E [E00002549, E00002550], K [K001097262-K001097266], P [P02272032]). 
= Ferula koelzii Rech. f. et Riedl, 1963, in Biol. Skr. 13, 4 (Symb. Afghan. 5): 70.

Type: AFGHANISTAN. "NW: Sangcharak: Safedsang, N. Qala Shahar. 23 IX 1939. Koelz 13997" (holotype US! [US00289206]).

Distribution in MA \& K: Tajikistan (Kŭhistani Badakhshan).

Distribution in other Asian countries: Pakistan, Afghanistan.

Distribution area: Central and South-Western Asia.

Ferula nevskii Korovin, 1937, Trudy Bot. Inst. Akad. Nauk SSSR (ser. 1 Fl. Sist. Vyssh. Rast.) 4: 270.

Type: TURKMENISTAN. "[Asia Media] Inter arbores Sabinae seraschanicae in montibus Kuhitang prope pag. Chodsha-i-fil. 27 VI 1931. Nevski 484" (holotype LE! [LE00051848]); "ad marginem inferiorem zonae Sabinae seraschanicae in montibus Kuhitang supra pagum Kuhitang. 14 VII 1931. Nevski" (syntype LE).

Distribution in MA \& $\mathbf{K}$ : Uzbekistan (Surxondarya d.), Turkmenistan (Lebap vel.). Endemic.

Distribution area: South-Western Asia.

Ferula nuda Spreng., 1818, Umbell. Sp.: 81, tab. 7, fig. 15 .

Type: KAZAKHSTAN. "In Sibiria [A Pallasio mis sa semina in horto Mayeri Sedinensis hanc plantam producerunt]".

Distribution in MA \& K: Kazakhstan (W: W Kazakhstan prov., Atyrau prov., Aktobe prov., Mangystau prov.; N: Akmola prov.; Central: Karagandy prov., Kzyl-Orda prov.), Uzbekistan (Kara-kalpak).

Distribution in other Asian countries: Russia.

Distribution area: Asia (Central), Europa.

Ferula nuratavica Pimenov, 1978, Byull. Moskovsk. Obshch. Isp. Prir. Otd. Biol. 83(4): 151.

$\equiv$ Silaus popovii Korovin, 1927, Byull. SredneAsiatsk. Gosud. Univ. 15: 52 [ Sched. Herb. Fl. Asia. Med. N 325]

$\equiv$ Johrenia popovii (Korovin) Korovin, 1959, in Vvedensky (ed.), Fl. Uzbekist. 4: 399.

$\equiv$ Silaum popovii (Korovin) M. Hiroe, 1979, Umbelliferae World: 772.

Type: UZBEKISTAN. "Montes meridionales: Sogdiano-Transoxanae. Ad abrupta calcarea montium Nura-tau in loco Jang-ogly dicto. $08 \mathrm{VI}$ 1926. Popov 325" (lectotype TASH! [TASH002337]; designated by Schischkin (1950: 548); isolectotypes B [B 10 0367274], BP!, BR [BR0000005623860], C! [C10008580], G! [G00359802, G00359803], K! [K001097274], LE! [LE01066093, LE01066094], MHA!, MO [MO-345419], MW! [MW0593905], NY [NY00406275], P [P03224545], S! [S-G-5676], Z [Z000028773).

Distribution in MA \& K: Uzbekistan (Navoi prov., Samarqand prov.). Endemic.

Distribution area: South-Western Asia.

Note:-Korovin (1927b) described the species on the basis of one series of specimens, edited in Sched. Herb. Fl. Asia Med., as exciccates, but he did not show what specimen, kept in what herbarium, was selected by him as a lectotype. Schischkin (1951: 548) showed this at the first time. A conbination of Korovin's (1927) and Schischkin's (1951) data seems to be a sufficient lectotypification of the species.

Ferula oopoda (Boiss. et Buhse) Boiss., 1872, Fl. Orient. (Boissier) 2: 984.

$\equiv$ Peucedanum oopodum Boiss. et Buhse, 1860, Nouv. Mém. Soc. Imp. Naturalistes Moscou 12: 100.

Type: AZERBAIDZHAN. "Beim Salzbergwerk in der Nahe von Nachtschewan, in einer Schlucht. 11 V 1847. Buhse 295/4" (lectotype G-BOIS!; designated as "typus" by Chamberlain \& Rechinger (1987: 421), isolectotypes E [E00433794] - photo], $\mathrm{P}$ [P02272182]).

= Ferula badghysi Korovin, 1947, Ill. Monogr. Ferula: 67, tab. 43, fig. 2.

Type: TURKMENISTAN. "Turcomania, SaryJasy. 08 VI 1916. Androssov" (holotype TASH! [TASH002379-TASH002384]).

= Ferula reza-dadjoua Parsa, 1966, Fl. Iran. 9: 185, fig. 62.

Type: IRAN. "Central Iran: Azizabad of Kashan. VI 1955. Parsa 10036" (holotype Herb. Parsa).

Distribution in MA \& K: Turkmenistan (Balkan prov., Akhal prov., Lebap prov., Mary prov.).

Distribution in other Asian countries: Iran, Azerbaidzhan, Armenia, Iraq.

Distribution area: South-Western Asia.

Ferula ovczinnikovii Pimenov, 1983, in Vvedensky (ed.), Opred. Rast. Sred. Azii 7: 377, 309.

Type: TAJIKISTAN. "Jugum Seravschanicum, steppae lapidosae in NE declivi prope ostium fl. Kschtut, 1550 m. 07 VI 1932. Ovczinnikov \& Slobodov" (holotype LE! [LE00051856]). 
- Ferula lehmannii auct. non Boiss.: Franch., 1883, Ann. Sci. Nat., Bot., sér. 7, 16: 297.

Distribution in MA \& K: Tajikistan (Sughd prov.), Uzbekistan (Samarqand prov.). Endemic.

Distribution area: South-Western Asia.

Ferula ovina Boiss., 1845, in Hohenacker (ed.). P1. Pers. Austr. № 283; id. 1872, F1. Orient. (Boissier) 2: 986).

EPeucedanum ovinum (Boiss.) Boiss., 1846, Diagn. P1. Orient. ser. 1, 6: 61.

Type: IRAN. "Persia austr., in rupestribus prope ruinas u. Persepolitanas, 22 IV 1842, Kotschy 283" (lectotype G-BOIS!; designated by Pimenov \& Jacquemoud in Kurbonov \& Pimenov, 2016: 1224); id., 2020: 53), isolectotypes BM! [BM000885452, BM000944771], C! [C10008441, C10008442), FI! [FI004421, FI014991], FR [FR0038111], G! [G00366721, G00366727, G00366745], GOET [GOET011338], HAL [HAL003647], JE! [JE0006704], K! [K000568180, K000568181], L! [L2576875], LE!, LIV!, M [M0173053, M0173054], MO [MO-345402, MO-345443, MO-345444], P! [P02272185 - P02272189, P04102870], S! [S-G2698], TI!, US [US00127482], W [W0053227, W0053228, W0053229, W-Rchb. 1889-0018448, W-Rchb. 1889-0018449], WAG [WAG0004949, WAG0004950]).

= Peucedanum thomsonii C. B. Clarke, 1879, in F1. Brit. India (J. D. Hooker) 2: 711.

Type: INDIA. "Kashmir, alt. 5000-9000 ft., Kishtwar. 10 V 1848. Thomson" (lectotype K! [K001097258], designated here by Pimenov; isolectotypes CAL, G! [G00366775], K! [K001097256, K001097257, K001097259], P! [P00752995], W! [W0053227]); “Kashmir, Banahal, Thomson" (syntypes K! [K01097250, K001097261]).

= Ferula pachycarpa Korovin 1935, in N.V. Pavlov (ed.), 1835, Fl. Centr. Kazakh. 2: 532, 537, nom. inval. (Art. 39.1).

=Ferula microcarpa Korovin, 1947, Ill. Monogr. Ferula: 58, tab. 33, fig. 2.

Type: KAZAKHSTAN. "Alatau Dshungariensis, distr. Kapal, Baj-Gazy. 11 VI 1917. Titov" (holotype TASH! [TASH002462]; isotypes LE [LE00051184], TASH! [TASH002463]).

= Ferula stylosa Korovin, 1947, Ill. Monogr. Ferula: 58, tab. 34, fig. 2.

Type: KAZAKHSTAN. "Mont. Tschu-ilienses, in loco Ajderke, Uzun-bulak, declivia calcarea. $18 \mathrm{VI}$ 1926. Titov 676" (holotype TASH! [TASH002497]).
= Ferula lapidosa Korovin, 1947, Ill. Monogr. Ferula: 59, tab. 35, fig. 1.

Type: KYRGYZSTAN. "Kirghyzstan, in angust. Buam, prope pontem medium, rubroarenosa gypsacea. 27 VII 1926. Abolin 639" (holotype TASH! [TASH002450, TASH002451]; isotypes TASH [TASH002454-TASH002456]).

Distribution in MA \& K: Kazakhstan (W: Mangystau prov.; NE: E Kazakhstan prov.; Central: Karagandy prov.; S: S Kazakhstan prov., Zhambyl prov.; SE: Almaty prov.), Kyrgyzstan (Batken prov., Jalal-Abad prov., Ysyk-köl prov., Naryn prov., Osh prov., Talas prov., Chüh prov.), Tajikistan (Kŭhistani Badakhshan, Sughd prov., Khatlon prov., Dushanbe prov.), Uzbekistan (Navoi prov., Dzhizak prov., Samarqand prov., Qashqadarya prov., Surxondarya prov., Toshkent prov.), Turkmenistan (Lebap prov.)

Distribution in other Asian countries: China, India, Pakistan, Afghanistan, Iran, Saudi Arabia, Yemen, Israel, Jordan.

Distribution area: Central \& South-Western Asia.

Ferula pachyphylla Korovin, 1947a, Ill. Monogr. Ferula: 54, tab. 28, fig. 2.

Type: KAZAKHSTAN. "Kara-tau, pars australis, Mt. Ak-murun, declivum lapitosum. 06 VI 1934. Tschilikina \& Volkova 96" (neotype TASH! [TASH002474], designated here).

Distribution in MA \& $\mathbf{K}$ : Kazakhstan ( $\mathrm{S}$ : $\mathrm{S}$ Kazakhstan prov., Zhambyl prov.). Endemic.

Distribution area: South-Western Asia.

Note: The type, designated by Korovin ("Asia Media, Karatau, Boroldaj. Tschlilikina"), was not been found in Tashkent. Neotype was separated among the gathertings of the same collector in TASH.

Ferula pallida Korovin, 1947a, Ill. Monogr. Ferula: 60, tab. 37, fig. 1.

Type: KAZAKHSTAN. "Prov. Syr-Darja, distr. Chimkent, SW pars montium Maisur-ata, $15 \mathrm{~km}$ ad stationem Montaitasch, declivia septentrionalia. 02 VI 1923. Simonova 329, 330" (holotype TASH! [TASH002475, TASH002476]).

Distribution in MA \& $\mathbf{K}$ : Kazakhstan ( $\mathrm{S}$ : S Kazakhstan prov., Zhambyl prov.), Kyrgyzstan (Talas prov.), Uzbekistan (Toshkent prov.). Endemic.

Distribution area: South-Western Asia.

Ferula paniculata (Ledeb.) Ledeb. 1844, Fl. Ross. (Ledeb.) 2: 305. 
$\equiv$ Peucedanum paniculatum Ledeb. 1829, Fl. Altaic. 1: 310. 1829, non Loisel. (1828), nom. illeg. (Art. 53.1).

$\equiv$ Peucedanum soongaricum $\mathrm{G}$. Don, 1834 . Gen. Hist. 3: 332, non Ferula songarica Pall. ex Schult., 1820 .

$\equiv$ Peucedanum feruloides Steud., Nomencl. Bot., ed. 2, 2: 311. 1841, nom. illeg. (Art. 52.1).

$\equiv$ Peucedanum pyramidatum Kar. \& Kir.1842, Bull. Soc. Imp. Naturalistes Moscou 15: 366, nom. illeg. (Art. 52.1).

$\equiv$ Ferula feruloides Korovin,1947a, Ill. Monogr. Ferula: 77, nom. illeg. (Art. 52.1).

$\equiv$ Ferula pyramidata Korovin, 1949, Izv. Akad Nauk Kaz. S.S.R. 52(3): 49 nom. illeg. (Art. 52.1).

Type: Kazakhstan. "In subsalsis deserti soongoro-kirghisici inter Irtysch \& montes Dolen-kara nec non in regione occidentali, C. A. Meyer 235" (lectotype LE [LE00052139!], designated by Pimenov, 2017, in Turczaninowia 20, 2: 143); isolectotypes CGE!, LE [LE00052140], P [P02272141, P02272142, P02272143]).

Distribution in MA \& K: Kazakhstan, China, Mongolia.

Distribution area: Central Asia

Note: The nomenclatural details see in Sennikov A. N. \& Pimenov M. G., Nomenclatural notes on four species of Umbelliferae (Apiaceae) in Middle Asia. Annales Botanicae fennicae (in press)

Ferula penninervis Regel et Schmalh., 1878, Trudy Imp. S.-Peterburgsk. Bot. Sada 5(2): 591.

Type: KAZAKHSTAN. "In Turkestaniae montibus karatavicis prope Karagus, A. Regel" (syntypes K!, LE! [LE00051861-LE00051864]); UZBEKISTAN. "Iter Turkestanicum, Tschirtschikthal, 4-6000', 1876, A. Regel" (lectotype LE! [LE00051867], designated by V. M. Vinogradova 2000: 93); isolectotypes G! [G00366768], K [K001097206], LE [LE00051865, LE00051866], P [P02272190, P02272191], US [US00517438]).

= Ferula olgae Regel et Schmalh., 1882, Izv. Obshch. Ljubit. Estestv. 34(2) (Descr. P1. Nov. Rar. Fedtsch.): 36.

Type: TAJIKISTAN. "In Kokaniae valle fluvii Sarawschan prope Obburden 6000' alt. O. A. Fedtschenko", (syntype LE?); KYRGYZSTAN. "In montibus Ktchi-alai 8000' alt. 27 VII 1871. O. A. Fedtschenko" (lectotype LE! [LE00051851], designated by V. M. Vinogradova 2000: 93); "Kokand khanate, Inter Karakasuk et Schagimardan 4500-6000' alt. O. Fedtschenko" (syntype LE [LE00051853]).
$=$ Ferula kaschkarovii Korovin, 1947a, Ill. Monogr. Ferula: 70, tab. 46, fig. 2.

Type: KYRGYZSTAN. "Fl. Ujunkur, 13 [Fergana, Ujunkur-su, affluxio Kara-su]. 31 VII 1926. Korovin 812, 814, 815, 817" (holotype TASH! [TASH002428-TASH002431])

Distribution in MA \& K: Kazakhstan (S Kazakhstan prov., Zhambyl prov.), Kyrgyzstan (Batken prov., Jalal-Abad prov., Ysyk-köl prov., Naryn prov., Osh prov., Talas prov., Chüh prov.), Tajikistan (Sughd prov.), Uzbekistan (Navoi prov., Dzhizak prov., Samarqand prov., Toshkent prov., Namangan prov.). Endemic.

Distribution area: South-Western Asia.

Ferula pimenovii Lazkov, 2006, Bot. Zhurn. (St. Perersburg) 91(9): 1410.

Type: KYRGYZSTAN. "Jugi Chatkalici brachia australis, ad meridiem ab pago Sumsar, in gypsaceis. 25 VII 2005. Lazkov" (holotype LE! [LE00051869]; isotypes FRU!, K! [K001097273], LE!, MW! [MW0594016]).

Distribution in MA \& K: Kyrgyzstan (JalalAbad prov.). Endemic.

Distribution area: South-Western Asia.

Ferula plurivittata Korovin, 1947a, Ill. Monogr. Ferula: 25, tab. 3, fig. 1.

Type: TURKMENISTAN. "Kasanshik, angustis montis Kjuren-dadh, 29 IV 1889, Antonov" (holotype LE! [LE00051876]; isotypes LE [LE00051871, LE00051875, LE00051877]).

Distribution in MA\& K: Turkmenistan (Balkan prov.). Endemic.

Distribution area: South-Western Asia.

Ferula potaninii Korovin [in Pavlov, 1935, Fl. Centr. Kazakh. 2: 532, 537, nom. inval., descr. ross.] 1947, Ill. Monogr. Ferula: 56, tab. 30, fig. 2.

Type: KAZAKHSTAN. "Asia Media, montes Tarbagatai [District Semipalatinsk, praemontia jugi Tarbagatai], Potanin" (holotype TK!).

Distribution in MA \& K: Kazakhstan (NE: E Kazakhstan prov.; SE: Almaty prov.).

Distribution in other Asian countries: China, Mongolia.

Distribution area: Central Asia.

Ferula prangifolia Korovin, 1947, Ill. Monogr. Ferula: 53, tab. 26, fig. 1.

Type: UZBEKISTAN. "Asia Media, TianSchan occidentalis, m. Tschimgan Minor. 15 VII 1926. Baranov" (holotype TASH - not found); 
"Syr-Darya prov., Toshkent distr., in declivo montis Tschimgan Minoris versus riv. Chadalak. 29 VII 1921. Drobov 214" (neotype TASH [TASH002478TASH002481], designated here).

Distribution in MA \& K: Kazakhstan ( $\mathrm{S}$ : $\mathrm{S}$ Kazakhstan prov.), Uzbekistan (Toshkent prov.). Endemic.

Distribution area: South-Western Asia.

Ferula pratovii F. O. Khass. et I. I. Maltzev, 1990, Dokl. Akad. Nauk Uzbeksk. SSR 8: 48.

Type: UZBEKISTAN. "Pamiroalaj occidentalis. Jugum Hissaricum. Vallis fl. Kyzyl-darja, prope pagum Vuary, $2400 \mathrm{~m}$ s. m. 30 VII 1989. Khassanov 448" (holotype TASH! [TASH002485]; isotypes LE, TASH! [TASH002482-TASH002484, TASH002486]).

Distribution in MA \& $\mathrm{K}$ : Uzbekistan (Qashqadarya prov.). Endemic.

Distribution area: South-Western Asia.

Note: The isotype of the species, transmitted by the authors to LE, is apparently not scanned yet.

Ferula renardii (Regel et Schmalh.) Pimenov, 1981, in S. K. Cherepanov, Sosud. Rast. SSSR: 21.

$\equiv$ Peucedanum renardii Regel et Schmalh 1878 , Trudy Imp. S.-Peterburgsk. Bot. Sada 5(2): 596.

$\equiv$ Talassia renardii (Regel et Schmalh.) Korovin, 1963, in Pavlov (ed.), Fl. Kazakhst. 6: 385.

Type: KYRGYZSTAN. "In Turkestaniae montibus Alexander in trajectum Karabura, 8000' alt., 1876, A. Regel" (lectotype LE [LE00052146]; isolectotype LE [LE00052147]; designated here by Pimenov; isolectotypes K! [K001097272]).], W photo).

= Peucedanum talassicum Korovin, 1947, Not. Syst. Herb. Inst. Bot. Zool. Acad. Sci. Uzbekistan 8: 7.

= Ferula talassica (Korovin) Pimenov, 1981, in S. K. Cherepanov, Sosud. Rast. SSSR: 21.

Type: KYRGYZSTAN. "Tian-Schan, montes Talassici, ad cursum fl. Simbili. 01 VIII 1922. Baranov 846" (holotype TASH! [TASH002558]).

Distribution in MA \& K: Kazakhstan (S: S Kazakhstan prov.), Kyrgyzstan (Jalal-Abad prov., Talas prov.), Uzbekistan (Toshkent prov.). Ende mic.

Distribution area: South-Western Asia.

Ferula rubroarenosa Korovin, 1947a, Ill. Monogr. Ferula: 59, tab. 39, fig. 1.

Type: KYRGYZSTAN. "Fergana orientalis, distr. Dzhelalabad, systema fl. Kugart-su, in cacuminibus Aschi-sai, in saxo rubroarenoso cretaceo. 17 VI 1927. Korovin 64, 66, 67" (holotype TASH! [TASH002488-TASH002490]).

= Ferula mollis Korovin, 1947a, Ill. Monogr. Ferula: 63, tab. 27, fig. 1.

Type: UZBEKISTAN. "Prov. Fergana, distr. Andishan, montes prope pag. Kenpyr-abad, 27 V 1916, Androssov 504" (lectotype LE! [LE00051846]; designated here; isolectotypes LE [LE00051845, LE00051847]).

$=$ Ferula latiloba Korovin, 1947a, I1l. Monogr. Ferula: 63, tab. 39, fig. 2.

Type: TAJIKISTAN. "Prov. Samarkand, distr. Chodshent, montes Mogoltau, Mt. Spa, in calcareis. 24 VI 1923. Popov \& Vvedensky 268, 269" (holotype TASH! [TASH002454-TASH002456]).

Distribution in MA \& K: Kyrgyzstan (Batken prov., Osh prov.), Tajikistan (Sughd prov.), Uzbekistan. (Samarqand prov., Qashqadarya prov., Surxondarya prov.). Endemic.

Distribution area: South-Western Asia.

Note: Holotype mounted on three sheets.

Ferula samarkandica Korovin, 1947a, Ill. Monogr. Ferula: 44, tab. 18, fig. 2.

Type: UZBEKISTAN. "Montes Samarkandici, prope pag. Ak-sai, 23 V 1931, Butkov 62" (holotype TASH! [TASH002491-TASH002494].

Distribution in MA \& K: Kazakhstan (S: S Kazakhstan prov.), Kyrgyzstan (Batken prov., JalalAbad prov.), Tajikistan (Sughd prov.), Uzbekistan (Dzhizak prov., Qashqadarya prov., Namangan prov., Samarqand prov., Surxondarya prov., Toshkent prov.). Endemic.

Distribution area: South-Western Asia.

Ferula schtschurowskiana Regel et Schmalh., 1882, Izv. Obshch. Ljubit. Estestv. 34(2) (Descr. Pl. Nov. Rar. Fedtsch.): 34.

$\equiv$ Dorema schtschurowskianum (Regel et Schmalh.) Koso-Pol., 1921, Bot. Mater. Gerb. Glavn. Bot Sada R.S.F.S.R. 2(1): 87.

Type: UZBEKISTAN. "In Turkestaniae deserto Kisil-Kum prope m. Ak-Kurai, $06 \mathrm{~V}$ 1871, O. A. Fedtschenko" (holotype LE! [LE00051889]; isotype LE [LE00051890]).

= Dorema serratum Aitch. et Hemsl., 1888, Trans. Linn. Soc. London, Bot. 3(1): 70, tab. 28.

Type: AFGHANISTAN. "Afghanistan, Kharez Ilias, 16 V 1885, Aitchison 476"; "Afghanistan, Karez. 25 VII 1885. Aitchison 718”.

Distribution in MA \& K: Kazakhstan (S: S Kazakhstan prov., Zhambyl prov.), Kyrgyzstan (Batken prov.), Tajikistan (Sughd prov.), Uzbekistan 
(Buxoro prov., Navoi prov., Dzhizak prov., Samarqand prov., Qashqadarya prov., Surxondarya prov., Sirdarya prov., Toshkent prov.), Turkmenistan (Akhal prov., Mary prov.).

Distribution in other Asian countries: Afganistan, Iran.

Distribution area: Central and South-Western Asia.

Ferula seravschanica Pimenov et Yu. V. Baranova, 1983, in Vvedensky (ed.), Opred. Rast. Sred. Azii 7: 376, 289.

Type: TAJIKISTAN. "Tadjikistania, jugum Turkestanicum, valle fl. Zarafschan in gypsaceis prope Gusaribad. 04 VII 1976. Baranova \& Kljuykov 918" (holotype MW! [MW0594018]; isotypes LE! [LE00051904, LE00051905], MW! [MW0594017]).

Distribution in MA \& K: Tajikistan (Sughd prov.). Endemic.

Distribution area: South-Western Asia.

Ferula sibirica Willd. 1798, Sp. Pl. (ed. 4) 1(2): 1411.

$\equiv$ Ferula peucedanifolia Willd. ex Spreng., 1818, Sp. Umbell.: 87; 1820, in Syst. Veg. ed. 15 bis Roemer et Schultes) 6: 592, nom. illeg.

$=$ Dardanis sibirica (Willd.) Raf. 1840, Good Book: 57

$\equiv$ Peucedanum cephalotes Boiss., 1844, Ann. Sci. Nat., sér. 3, 1: 314.

$\equiv$ Peucedanum pallasii Cusson ex Ledeb., 1844, Fl. Ross. (Ledebour) 2: 272, nom. inval. (Art. 36.1), in syn.

$\equiv$ Ferula pallasii [Cusson ex Ledeb.] KosoPol., 1925, Byull. Obshch. Estestvoisp. Voronezsk. Gosud. Univ. 1: 37, in obs., nom. inval. (Art. 32.1?) et 1925, Byull. Obshch. Estestvoisp. Voronezsk. Gosud. Univ. 1: 92, in clavi.

$\equiv$ Soranthus peucedanifolius (Willd.) Woronow, 1931, Trudy Bot. Sada Akad. Nauk URSS 43(2) (Flora Yugo-Vost. 5): 823.

$\equiv$ Soranthus sibiricus (Willd.) Korovin, 1947, Ill. Monogr. Ferula: 81.

Type: EUROPE, RUSSIA. "In Sibiria locis arenosis humidiusculis inter Wolgam et Jaikam, Pallas" (lectotype B-Willd! [B-W-05772-01 0, B-W-05772-02 0, B-W-05772-03 0]; designated by Pimenov, 2017, in Turczaninowia 20, 2: 146); isolectotype HAL [HAL0036472, HAL0036473]).

= Soranthus meyeri Ledeb., 1829 (Maj-Dec), Icon. Pl. 1: 20, tab. 82; ejusd. 1829 (Nov.-Dec.), Fl. Altaic. (Ledebour) 1: 345.1829 (Nov-Dec.)
$=$ Seseli meyeri (Ledeb.) Dietr. 1840, Syn. Pl. 2: 956.

$=$ Ferula meyeri (Ledeb.) Bunge, 1852, Beitr. Fl. Russl. (Lehm. Rel. Bot.): 131; 1851, Mém. Acad. Imp. Sci. St.-Petersbourg Divers Savans. 7: 307.

Type: KAZAKHSTAN. "In arenosis ad fl. Irtysch non procul a lacu, qui Noor-Saisan vocatur et ad fl. Bekau, Meyer 265" (lectotype LE!; designated by Pimenov, 2017, in Turczaninowia 20, 2: 146), isolectotype P! [P02272175, P02272176]).

= Ferula capillifolia Stschegl., 1854, Bull. Soc. Imp. Naturalistes Moscou 27(1): 168, nom. inval.

Type: KAZAKHSTAN. "In sabulosis ad fl. Irtysch. 01 VII 1843. Karelin" (holotype LE! [LE0100655850]).

- Ferula nodiflora auct. non L.: Pall., 1776, Reise russ. Reich 3: 735.

Type: KAZAKHSTAN. "Inter collem arenosis, locis humidioribus, copiosissime supra fortelitium Iamyschewa. VI 1771. Pallas".

Distribution in MA \& K: Kazakhstan (W: W Kazakhstan prov., Aktobe prov.; N: Akmola prov; NE: E Kazakhstan prov.; Central: Karagandy prov., Kzyl-Orda prov.).

Distribution in other Asian countries: Russia, China.

Distribution area: Asia (Central), Europa (E).

Ferula soongarica Pall. ex Spreng., 1820, in Syst. Veg. ed. 15 bis (Roemer \& Schultes) 6: 598.

$\equiv$ Peucedanum aureum Spreng., 1824, Syst. Veg. (ed. 16) 1: 911, p. p.

Type: RUSSIA. "Siberia, Pallas" (syntypes B-Willd [B-W 05774-01 0], BM?, M! [M0173051]).

= Peucedanum elatum Ledeb., 1829, Fl. Altaic. (Ledebour) 1: 304.

Type: KAZAKHSTAN. "[Altai] In declivibus herbedis ubique [Ad fl. Gramatucha], Ledebour 252" (lectotype LE! [LE00052120], designated by V. M. Vinogradova 2005: 963); isolectotypes C [C10008444, C10008445], CGE!, HAL, P [P02272203， P02272204， P022722067]); “In declivibus apricis herbedis frequens, 1826, Bunge" (syntypes LE!, P [P02272205, P02272206, $\mathrm{P} 02272215, \mathrm{PH}$ [PH00023031]).

= Ferula ehrenbergii H. Wolff, 1924, Repert. Spec. Nov. Regni Veg. 19: 310.

Type: RUSSIA. "Sibiria occidentalis, loco non indicato, Ehrenberg".

$=$ Ferula soongarica Pall. ex Spreng. var. arida Korovin, 1947, Ill. Monogr. Ferula: 71.

$=$ Ferula arida (Korovin) Korovin, 1963, in Pavlov (ed.), Fl. Kazakhst. 6: 411, tab. 57, fig. 2. 
Type: KAZAKHSTAN. "In regione desertorum arida".

= Ferula soongarica Pall. ex Spreng. subsp. mongolica V. M. Vinogr. et Kamelin, 1986, Novit. Syst. P1. Vasc. 23: 101.

= Ferula mongolica (V. M. Vinogr. et Kamelin) V. M. Vinogr. et Kamelin, 1990, Novit. Syst. P1. Vasc. 27: 118

Type: MONGOLIA. "Declivium montium in angustis Botchon. 18 VII 1899. Klementz" (holotype LE!).

Distribution in MA \& K: Kazakhstan (W: Aktobe prov.; N: N Kazakhstan prov., Pavlodar prov., Akmola prov.; NE: E Kazakhstan prov.; Central: Karagandy prov.; S: Zhambyl prov.; SE: Almaty prov.).

Distribution in other Asian countries: Russia, China, Mongolia.

Distribution area: Northern and Central Asia.

Ferula subtilis Korovin, 1947, Ill. Monogr. Ferula: 44, tab. 20, fig. 1.

Type: KYRGYZSTAN. "Asia Media, vall. Ferganensis inter Utsch-Kurgan et Arpalyk ad loca sicca lapidosa. 10 VI 1913. Desjatova 928" (holotype LE! [LE00051911]; isotypes LE [LE00051910, LE00051912]).

Distribution in MA \& K: Kyrgyzstan (Osh prov.). Endemic.

Distribution area: South-Western Asia.

Ferula sugatensis Bajtenov, 1970, Vestnik Akad. Nauk Kazak. SSR 7: 71.

Type: KAZAKHSTAN. "Ala-Tau transiliensis (pars orientalis), vallis Sjugatin, in maculis salsas. 15 VI 1955. Goloskokov" (holotype AA!; isotype LE! [LE00051909]).

Distribution in MA \& K: Kazakhstan (SE: Almaty prov.). Endemic.

Distribution area: South-Western Asia.

Ferula sumbul (Kauffm.) Hook. f., 1875, Bot. Mag. 101: tab. 6196.

三Euryangium sumbul Kauffm., 1870, Izv. Obshch. Ljubit. Estestv. 8(1): 392, tab. 1, 2.

$\equiv$ Peucedanum sumbul (Kauffm.) Baill., 1879, Hist. Pl. (Baillon) 7: 186.

Type: TAJIKISTAN. "Described on the basis of plants cultivated in BG of Moscow University from the roots collected by A. P. Fedtschenko in Magian Mts. near Pendzhikent, 28 V 1869" (holotype LE!).

$=$ Peucedanum pseudoreoselinum Regel et Schmalh., 1882, Izv. Obshch. Ljubit. Estestv. 34(2) (Descr. Pl. Nov. Rar. Fedtsch.): 36.
$=$ Ferula pseudoreoselinum (Regel et Schmalh.) Koso-Pol., 1925, Byull. Obshch. Estestvoisp. Voronezsk. Gosud. Univ. 1: 94, p. p.

Type: TAJIKISTAN. "In Turkestaniae regione fluvii Sarawschan in valle fluvii Iskander, 7000' alt., O. Fedtschenko" (syntype LE); "[In Turkestaniae] in angustiis Passrut in regione fluvii Sarawschan 6-10000' alt., O. A. Fedtschenko" (syntype LE); "[In Turkestaniae] ad lacum Iskander, 8000'. 15 VI 1870. O. A. Fedtschenko" (lectotype LE! [LE00052137]: designated here by Pimenov).

= Ferula moschata Koso-Pol., 1925, Byull. Obshch. Estestvoisp. Voronezsk. Gosud. Univ. 1: 94, nom. inval. (Art. 33.3).

Type: UZBEKISTAN/TAJIKISTAN. "Mont. Zeravschanici in valle fl. Zeravschan; mont. Turkestanicae in valle fl. Sansar; Mont. Hissarici in valle fl. Jakkabag" (syntypes).

= Ferula urceolata Korovin, 1964, Novit. Syst. Pl. Vasc. [1] 1964: 249.

Type: TAJIKISTAN. "Asia Media, Tajikistania centralis, jigum Karateginense, prope pag. Ramit, in valle fl. Tavischi, alt. 2300 m. s. m. 14 VIII 1962. Strishova 2018" (holotype TAD!; isotype LE!).

Distribution in MA \& K: Tajikistan (Sughd prov., Dushanbe prov.), Uzbekistan (Dzhizak prov., Qashqadarya prov., Surxondarya prov.). Endemic.

Distribution area: South-Western Asia.

Ferula syreitschikowii Koso-Pol., 1922, Bot. Mater. Gerb. Glavn. Bot. Sada R.S.F.S.R. 3(18): 71.

Type: KAZAKHSTAN. "In prov. Semiretschensk (Heptapotamia), distr. Kopal, in ripa fluminis Karatal inter loc. Kos-Tugaj et Naiman-mula, salsa rimosa takyr dicta. 03 VI 1913. Schischkin \& Genina" (syntypes LE! [LE00051913-LE00051917]).

= Ferula puberula Trautv. 1866, Bull. Soc. Imp. Naturalistes Moscou 39(2): 323, nom. illeg. (A 53), non Boiss. et Buhse (1860).

Type: KAZAKHSTAN. Ad lacum Balchasch. 13 VI 1841. Schrenk 349 (holotype LE! [LE00051886]), isotypes LE [LE00051880-LE00051884].

= Pastinaca olgae Regel et Schmalh., 1882, Izv. Obshch Ljubit. Estestv. 34(2) (Descr. Nov. Rar. Fedtsch.): 37.

Type: KAZAKHSTAN. "In Turkestatiae montibus Karak desertum Kisil-kum adjacentibus, $08 \mathrm{~V}$ 1871, O. A. Fedtschenko" (holotype LE! [LE00052192], designated by V. M. Vinogradova 2002: 139); "In Turkestania prope Chodschent. O. A. Fedtschenko" (syntype LE); "In Turkestaniae deserto prope Mai-bulak. Krause" (syntype LE).

Distribution in MA \& K: Kazakhstan (W: Aktobe prov.; NE: E Kazakhstan prov.; Central: 
Karagandy prov., Kzyl-Orda prov.; S: Zhambyl prov.; SE: Almaty prov.), Uzbekistan (Kara-kalpak).

Distribution in other Asian countries: China.

Distribution area: Central Asia.

Ferula szowitsiana DC., 1830, Prodr. (A. P. de Candolle) 4: 173.

$\equiv$ Peucedanum szowitsianum (DC.) Baill., 1879, Hist. Pl. (Baillon) 7: 186.

Type: IRAN. "In argillosis lapidosisque Persiae promontorii ad pagum Seidchodzi, distr. Khoi, prov. Aderbeizan, 20 V 1828, Szowits 294" (lectotype G-DC!; designated here by Pimenov; isolectotypes K! [K001097218, K001097219], LE!, M!, P! [P02272210, P02272211], S! [S12-21674], W! [W0053742]; "ibid. Szowitz 415" (syntypes K [K000568185], P [P02272212, P02272213]).

= Ferula microloba Boiss., 1872, Fl. Orient. (Boissier) 2: 989.

Type: IRAN. "In Persiâ ad Seidabad inter Teheran et Tabris, 06. VI 1859. Bunge" (syntypes GBOIS!, LE! [LE00015755]); "Prope Schahrud, 19 V 1858, Bunge" (lectotype G-BOIS!: designated by Pimenov et Jacquemoud (2020: 54); isolectotypes E [E00433774 - photo]), JE! [JE00002667], K! [K001097220], P! [P02272180, P02272181], S! [S12-21674]; "Seidabad. inter Teheran et Tabris. 06. 1858, Bunge" (syntypes G-BOIS, LE [LE00015755]).

= Ferula hirtella Boiss., 1872, Fl. Orient. (Boissier) 2: 993.

Type: IRAN. "Regio montana et alpina inter Nischapur et Mesched. VI-VII 1858. Bunge" (lectotype G-BOIS!; designated here by Pimenov \& Jacquemoud (2020: 56); isolectotypes P! [P02272158, P02272159], photo E [E00433776]).

= Ferula collina Freyn, 1900, Mém. Herb. Boissier 13: 25.

Type: TURKMENISTAN. "Regio transcaspica, ditio Aschabadensis: inter Anan et Giaurs, $02 \mathrm{~V}$ 1896, Brotherus 334" (lectotype BRNM!; designated by Pimenov et Sutorý 2014: 1106; isolectotype BRNM).

$=$ Ferula khorassanica Rech. f. et Aellen, 1952, Anz. Oesterr. Akad. Wiss., Math.-Naturwiss. K1. 89, 11: 174.

Type: IRAN. "Khorassan: inter Chenaran et Quchan, 1200-1300 m, Rechinger K. H. \& Rechinger F. 4695" (lectotype W! [[W 1963-0000629]; designated here by Pimenov; isolectotypes E [E002790 194], G! [G00366769]).

Distribution in MA \& K: Turkmenistan (Balkan prov., Akhal prov., Mary prov.).
Distribution in other Asian countries: Afghanistan, Iran, Azerbaidzhan, Armenia, Turkey.

Distribution area: South-Western Asia.

Ferula tadshikorum Pimenov, 1974, Byull. Glavn. Bot. Sada 94: 54.

Type: TAJIKISTAN. "Asia Media, Tajikist ania australis, jugum Sarsarjak, in valle fluvii Vachsch, ad trajectum Schar-Schar, in Acereto regelii, 1200 m s. m. 05 VII 1971. Pimenov 507" (holotype MW! [MW0594020, MW0594021]).

Distribution in MA \& K: Tajikistan (Khatlon prov., Dushanbe prov.), Uzbekistan (Surxondarya prov.), Turkmenistan (Lebap prov.).

Distribution in other Asian countries: Afghanistan.

Distribution area: South-Western Asia.

Ferula tatarica Fisch. ex Spreng., 1813, P1. Min. Cogn. Pug. 1: 27.

Type: RUSSIA. "Siberia".

= Peucedanum sibiricum Willd. 1798, Sp. P1. ed. 4 (Willdenow) 1 (2): 1406, non Ferula sibirica Willd.

$=$ Ferula sibirica (Willd.) Spreng., 1824, Syst. Veg. (ed. 16) 1: 915, nom. illeg., (Art. 53), non Ferula sibirica Willd. (1798), 1840, Good Book: 57.

Type: RUSSIA. "In Sibiria, Pallas" (lectotype B-Willd! [B-W05751-01-2, B-W05751-02 0], designated here by Pimenov; isolectotype HAL [HAL097783 -A \& B] sub Peucedanum sibiricum?).

Distribution in MA \& K: Kazakhstan (W: W Kazakhstan prov., Atyrau prov., Aktobe prov.; N: Kostonai prov., N Kazakhstan prov., Akmola prov., Pavlodar prov.; NE: E Kazakhstan prov.; Central: Karagandy prov.).

Distribution in other Asian countries: Russia.

Distribution area: Asia (Northern and Central), Europa (E).

Ferula taukumica Bajtenov, 1975, Bot. Mater. Gerb. Inst. Bot. Akad. Nauk Kazakhsk. SSR 9: 28.

Type: KAZAKHSTAN. "Cursus inferior fl. Ili, arenae Taukum, in demissis interjugalibus. $17 \mathrm{VI}$ 1970. Kurochkina" (holotype AA!).

- Ferula litwinowiana auct. non Koso-Pol.: Korovin, 1963, in Pavlov (ed.), Fl. Kazakhst. 6: 390, p. p.

Distribution in MA \& K: Kazakhstan (SE: Almaty prov.). Endemic.

Distribution area: Central Asia. 
Ferula tenuisecta Korovin, 1947a, Ill. Monogr. Ferula: 60, tab. 37, fig. 2.

Type: KAZAKHSTAN. "Fl. Kelte-Maschat [Tianschan occidentalis, ad declivia glareosa..., fl. Kelte-maschat pr. pag. Antonovka]. 16 V 1927. Linczevski \& Mokeeva 92" (holotype TASH! [TASH003714]).

Distribution in MA \& K: Kazakhstan (S: S Kazakhstan prov., Zhambyl prov.), Kyrgyzstan (Jalal-Abad prov.), Tajikistan (Sughd prov.), Uzbekistan (Buxoro prov., Navoi prov., Samarqand prov., Qashqadarya prov., Toshkent prov., Namangan prov.). Endemic.

Distribution area: South-Western Asia.

Ferula teterrima Kar. et Kir. 1842, Bull. Soc. Imp. Naturalistes Moscou 15: 363.

Type: KAZAKHSTAN. "In collibus lapidosis Songoriae prope fontem Sassyk-pastau, inter fluvios Ajagus et Lepsa. VI 1841. Karelin \& Kirilov 377" (lectotype LE! [LE00051922], designated by Korovin 1951: 91; isolectotypes LE [LE00051919LE00051921, LE00051923], MW! [MW0594031, MW0594032], TK).

= Ferula balchaschensis Bajtenov, 1970, Vestnik Akad. Nauk Kazak. SSR 7: 71.

Type: KAZAKHSTAN. "Regio Balchaschensis, in vallo ripario lacus arenoso schistose. 19 VI 1938. Dmitrieva" (holotype AA!).

Distribution in MA \& K: Kazakhstan (NE: E Kazakhstan prov.; SE: Almaty prov.).

Distribution in other Asian countries: China.

Distribution area: Central Asia.

Ferula transiliensis (Herder) Pimenov, 1981, in S. K. Cherepanov, Sosud. Rast. SSSR: 21.

三Peucedanum transiliense Herder, 1866, Bull. Soc. Imp. Naturalistes Moscou 39(3): 78.

三 Talassia transiliensis (Herder) Korovin, 1963, in Pavlov (ed.), F1. Kazakhst. 6: 384, tab. 52, fig. 1.

Type: KAZAKHSTAN. "Kurmetty-Passe im Alatau Transiliensis, in einer Hohe von 7000' [Kurmesty pass, Alatau transil.], Semenov" (lectotype LE! [LE00052171], designated here; isolectotypes LE [LE00052172, LE00052173]).

Distribution in MA \& K: Kazakhstan (S: Zhambyl prov.; SE: Almaty prov.), Kyrgyzstan (Batken prov., Jalal-Abad prov., Ysyk-köl prov., Naryn prov., Osh prov., Talas prov., Chüh prov.), Tajikistan (Sughd prov.), Uzbekistan (Toshkent prov.).

Distribution in other Asian countries: China.

Distribution area: Central and South-Western Asia.
Ferula tschimganica Lipsky ex Korovin, 1947, Ill. Monogr. Ferula: 54, tab. 29, fig. 1.

Type: UZBEKISTAN. "Mt. Chimgan, trajectus Peschany (arenosum). 17 VII 1897. O. A. Fedtschenko" (lectotype LE! [LE00051927, LE00051928], designated here; isolectotype LE [LE00051925, LE00051926]).

=Ferula minkwitziae Korovin, 1947, Ill. Monogr. Ferula: 53, tab. 27, fig. 2.

Type: KYRGYZSTAN. "Prov. Syr-Darja, distr. Aulie-Ata, jugum Ichkeletau, angustis Ulakan-kapka, saxa, 16 V 1909, Minkwitz 117' (holotype LE! [LE00051843]).

- Ferula ceratophylla auct. non Regel et Schmalh.: Korovin, 1926, Byull. Sredne-Asiatsk. Gosud. Univ. 14, Suppl. (Sched. Herb. Fl. As. Med. 10): N 244.

- Ferula karategina auct. non Lipsky ex Korovin: Korovin, 1951, in Schischkin (ed.), Fl. URSS 17: 107, p. p., quoad exc. HFAM N 244.

Distribution in MA \& K: Kazakhstan (S: S Kazakhstan prov.), Kyrgyzstan (Jalal-Abad prov., Talas prov.), Tajikistan (Sughd prov.), Uzbekistan (Toshkent prov.). Endemic.

Distribution area: South-Western Asia.

Ferula tschuiliensis Bajtenov, 1970, Vestnik Akad. Nauk Kazak. SSR 7: 71.

Type: KAZAKHSTAN. "Regio Dzhambulensis, in steppa inter Merke et Dzhambul. 10 VII 1949. N. V. Pavlov" (holotype AA!; isotype MW! [MW0594033]).

Distribution in MA \& K: Kazakhstan (S: Zhambyl prov.), Kyrgyzstan (Talas prov., Chüh prov.). Endemic.

Distribution area: Central and South-Western Asia.

Ferula tuberifera Korovin, 1947a, Ill. Monogr. Ferula: 43, tab. 13, fig. 2. [Herbarium Florae URSS N 6418]

Type: UZBEKISTAN. "Uzbekistania, brachia austro-occident. jugi Hissarici, Montes Tschulbair, pag. Oj-Borik, 26 IV 1928, Vvedensky 6418" (lectotype TASH! [TASH002498], 43); isolectotypes]; isotypes BM!, C! [C10005759], ERE [ERE0005558], G! [G00366800], LE! [LE0051930, LE00051931, LE00051932, LE00051933], LW [LW00214070], M! [[M0173197], MAK, MHA!, MICH [MICH1115191], MO [MO-345401], MW! [MW0594034], P [P00519032], S! [S-G-2700], TASH! [TASH002499-TASH002510], TNS!, US [US00664202], VAL [VAL10103], W!, WIS [WISv0255969WIS]). 
Distribution in MA \& K: Uzbekistan (Qashqadarya prov. Surxondarya prov.), Turkmenistan (Lebap prov.). Endemic.

Distribution area: South-Western Asia.

Note: See the note for Ferula kelifi

Ferula ugamica Korovin, 1947a, Ill. Monogr. Ferula: 66, tab. 42, fig. 1.

Type: UZBEKISTAN. "Ripa dextra fl. Angren, in cursu medio riv. Ak-sai, $\mathrm{N}$ ad Ablyk-sai, in rupibus. 20 VI 1924. Korovin 333, 336, 337, 339, 340, 341, 342, 343, 344, 345" (holotype TASH! [TASH002511-TASH002520]).

Distribution in MA \& K: Kazakhstan (S: S Kazakhstan prov.), Kyrgyzstan (Jalal-Abad prov.), Tajikistan (Sughd prov.), Uzbekistan (Toshkent prov.). Endemic.

Distribution area: South-Western Asia.

Ferula varia (Fisch., C. A. Mey. et Avé-Lall.) Trautv., 1866, Bull. Soc. Imp. Naturalistes Moscou 39(2): 325.

$\equiv$ Peucedanum varium Fisch., C. A. Mey. et AvéLall., 1843, Ind. Sem. Horti Petrop. 9, Suppl.: 15.

Type: KAZAKHSTAN. "In convallibus montium Chantau. 25 VI 1843. Schrenk 42" (lectotype LE! [LE00052175], designated here; isolectotypes LE [LE00052174, LE00052176, LE00052177]).

= Ferula schair Borszcz., 1860, Mém. Acad. Imp. Sci. St.-Petersbourg Divers Savants (7 ser.) 3(8): 37, tab. 6-8, "shair".

= Peucedanum schair (Borszcz.) Baill., 1879, Hist. Pl. (Baillon) 7: 186.

Type: KAZAKHSTAN. "In deserto sterilissimo argilloso-salso ex septemtrione fl. Ssyr-Darja inter locus Katschkan-Ssu et Aryss, 11 V 1851, Borszczow 751" (syntypes LE [LE001032129-LE001032136]).

Distribution in MA \& K: Kazakhstan (Central: Karagandy prov., Kzyl-Orda prov.; S: S Kazakhstan prov., Zhambyl prov.; SE: Almaty prov.), Kyrgyzstan (Chüh prov.), Uzbekistan (Kara-kalpak, Buxoro prov., Dzhizak prov., Navoi prov.). Endemic.

Distribution area: Central Asia.

Ferula vicaria Korovin, 1947a, Ill. Monogr. Ferula: 72.

Type: KYRGYZSTAN. "Prov. Fergana, montes Sary-tau. 24 VI 1920. Popov 624" (holotype TASH! [TASH002521]).

Distribution in MA \& K: Kyrgyzstan (Batken prov.), Uzbekistan (Fergona prov.). Endemic.

Distribution area: South-Western Asia.
Ferula violacea Korovin, 1964, Novit. Syst. P1. Vasc. [1] 1964: 250.

Type: TAJIKISTAN. "Asia Media, Tajikistania australis, distr. Baldshuan, systema fl. Talchak, prope pag. Dedy-Bul, 1160 m s. m. 04 VII 1956. Nikitin \& Czukavina 60" (holotype TAD!).

Distribution in MA \& K: Tajikistan (Kŭhistani Badakhshan, Khatlon prov.). Endemic.

Distribution area: South-Western Asia.

Ferula xeromorpha Korovin, 1947, Ill. Monogr. Ferula: 62, tab. 32, fig. 1.

Type: KAZAKHSTAN. "Praemontii collini W Tian-Schan in regione finem distr. Taschkent et Chimkent, montes Ala-tau, declivia Australia. 06 VI 1926. Jarmolenko 268" (holotype LE! [LE00051937, LE00051938]).

$=$ Ferula ligulata Korovin, 1947, Ill. Monogr. Ferula: 63, tab. 36, fig. 1.

Type: KAZAKHSTAN. "Culta in HB Univ. As. Med. e seminibus ad elevat. Alym-Tau".

Distribution in MA \& K: Kazakhstan (S: S Kazakhstan prov.). Endemic.

Distribution area: South-Western Asia.

Ferula xylorhachis Rech. f., 1940, Repert. Spec. Nov. Regni Veg. 48: 128.

Type: IRAN. "Khorasan: Im Schutt der Serpentinberge von Robat-Safid, zwischen Meshhed und Turbat-e Haidari, ca. 1700 m. 10-11.0 VII 1937. Rechinger 1529" (lectotype W! [W 19510011717]; designated here by Pimenov; isolectotypes BM [BM000944768], E! [E00433789 photo], K! [K000568179], LD! [LD183873], NY [NY004050954], S! [S-G-2701], US [US00127429], W [W1951-0011717]).

Distribution in MA \& K: Turkmenistan (Akhal prov.).

Distribution in other Asian countries: Iran.

Distribution area: South-Western Asia.

Ferulago subvelutina Rech. f., 1940, Repert. Spec. Nov. Regni Veg. 48: 129.

$\equiv$ Ferula subvelutina (Rech. f.) M. Hiroe, 1979, Umbelliferae World: 1525.

Type: IRAN. "Khorashan Persiae, steile steinige Hänge eines Tälchens in dem Bergland westlich von Budjnurd, jenseits der Passhöhe gegen Morawe Tappeh, $1100 \mathrm{~m}$... [In montibus inter Bidjnurd et Morawe Tappeh]. 26 VII 1937. Gauba [\& Rechinger] 1919" (holotype W [W 1951-0011714]; designated by Chamberlain (1987: 430); isotypes 
B! [B 10 0367192], BM! [BM000885412], E!, G, K! [K000685795], LD! [LD1227804], NY [NY00405956], S! [S-G-2702], US [US00127435]). $=$ Ferulago turcomanica Schischk. 1946, Bot. Zhurn. S.S.S.R. 31(6): 8.

$=$ Ferula turcomanica (Schischk.) M. Hiroe, 1979, Umbelliferae World: 1525.

Type: TURKMENISTAN. "Turcomania, in viciniis pagi Germab, in clivis lapidosis ad ripam fl. Germab. 02 VIII 1931. Linczevski" (holotype LE!).

Distribution in MA \& K: Turkmenistan (Balkan prov., Akhal prov.).

Distribution in other Asian countries: Iran.

Distribution area: South-Western Asia.

Foeniculum vulgare Mill., 1768, Gard. Dict. (ed. 8): Foeniculum N 1.

$\equiv$ Anethum foeniculum L., 1753, Sp. Pl.: 263.

三Ligusticum foeniculum (L.) Crantz, 1767, Cl. umbell. emend.: 82.

$\equiv$ Anethum rupestre Salisb., 1796, Prodr. stirp. Chap. Allerton: 168. 1796.

$\equiv$ Meum foeniculum (L.) Spreng., 1813, Neue Schr. Naturf. Ges. Halle 2, 1 (Pl. Umb. Prodr.): 32.

$\equiv$ Foeniculum foeniculum (L.) H. Karst., 1882, Deut. Fl. 9: 837, nom. inval. (Art. 23.4).

$\equiv$ Selinum foeniculum (L.) E. H. L. Krause, 1904, in Fl. Deutsch. (Sturm), ed. 2 12: 115.

$\equiv$ Seseli foeniculum (L.) Koso-Pol., 1916, Bull. Soc. Imp. Naturalistes Moscou, n. s. 29: 183.

Type: SOUTH EUROPA. "In Narbonne, Aremoriae, Maderae rupibus cretaceis, 106, Anethum 2" (lectotype BM-Cliff [BM000558385], designated by Jansen 1981: 25)

$=$ Foeniculum officinale All., 1785, Fl. pedem. 2: 25 .

Type: EUROPE, ITALY. "Circa Pinelorium. In comitatu Nicaeensi, et in provincia Uneliae".

= Anethum panmorium Roxb. ex Flem., 1810, Asiat. Res. 11: 156.

= Foeniculum panmorium (Roxb. ex Flem.) DC., 1830, Prodr. (A. P. de Candolle): 142.

Type: INDIA. "Plants, cultivated in Bengal".

= Ozodia foeniculacea Wight et Arn., 1834, Prodr. fl. Ind. orient. 1: 375.

Type: INDIA. "India Orientalis, Wight 1210" (lectotype E! [E00174688], designated here; isolectotypes NY, P [P03237720]).

$=$ Foeniculum commune Bubani, 1900, Fl. Pyren. (Bubani) 2: 372, "Feniculum", nom. illeg. (Art. 52.1).

$=$ Foeniculum vulgare Mill. f. pauciradiatum Nábĕlek 1923, Spisy Přir. Fak. Masarikovy Univ. 35: 127.
Type: EGYPT. "Arabia petraea, Wadi el Hsa inter El-Karac et Es-Sabak, inter rupes, alt. ca. 800 m. 16 VI 1909. Nábělek 467" (holotype SAV [SAV002348, SAV002349]).

Distribution in MA \& K: Kazakhstan (S: S Kazakhstan prov.), Tajikistan (Kühistani Badakhshan, Khatlon prov., Dushanbe prov.), Uzbekistan (Qashqadarya prov.), Turkmenistan (Balkan prov., Lebap prov.).

Distribution in other Asian countries: Russia, China, Korea, Philippines, Indonesia, Malaysia, Thailand, Myanmar, Bangladesh, Bhutan, Nepal, India, Pakistan, Afghanistan, Iran, Azerbaidzhan, Georgia, Armenia, Turkey, Iraq, Qatar, UAE, Saudi Arabia, Yemen, Lebanon, Syria, Israel, Jordan, Egypt (Sinai), Cyprus.

Distribution area: Asia (N, Central, E, SW, Mediterranean, W Tropical and E Tropical), Europa, Africa, N. America (adv.), S. America (adv.), Australia \& Oceania(adv.).

Galagania ferganensis (Korovin) M. G. Vassiljeva et Pimenov, 1983, in Vvedensky (ed.), Opred. Rast. Sred. Azii 7: 222.

三 Korovinia ferganensis Korovin, 1947, Not. Syst. Herb. Inst. Bot. Zool. Acad. Sci. Uzbekistan 8: 10.

Type: KYRGYZSTAN. "Tian-Schan, montes Ferganici prope p. Uzbek-gava. 02 VIII 1935. Kazatschkov" (holotype TASH! [TASH002559]).

Distribution in MA \& K: Kyrgyzstan (Batken prov., Jalal-Abad prov., Osh prov.), Tajikistan (Sughd prov., Khatlon prov., Dushanbe prov.), Uzbekistan (Namangan prov.). Endemic.

Distribution area: South-Western Asia.

Galagania fragrantissima Lipsky, 1900, Trudy Imp. S.-Peterburgsk. Bot. Sada 18(1): 63.

$\equiv$ Bunium fragrantissimum (Lipsky) Koso-Pol., 1916, Bull. Soc. Imp. Naturalistes Moscou, n. s. 29: 203, in adnot.

$\equiv$ Muretia fragrantissima (Lipsky) Korovin, 1926, Schedae Herb. Fl. As. Med. 10): 10.

Type: TAJIKISTAN. "Buchara, Hissar, Romit, 4100', 21 VII 1896, Lipsky 92” (lectotype LE! [LE00051634], designated by A. M. Geldykhanov 1992: 117; isolectotype LE! [LE00051635]; "Hissar, fl. Karatag, Pushti-miona, 23 VI 1897, Lipsky" (syntype LE! [LE00051638]); "Karatag, 29 VI 1896, Lipsky" (syntype LE! [LE00051642]); "Karatag, VII.1882, A. Regel" (syntype LE! [LE00051641]); "Dashnabad, 24 VI 1896, Lipsky" (syntype LE! [LE00051637]); "Inter Tian et Dagana, 03 VII 1899, Lipsky" (syntype LE! [LE00051636]); "Baldzhuan, 
Kalkat, decl. orient. Sangulak, 5000', 12 VII 1883, A. Regel" (syntype LE! [LE00051644]); "Fl. Jakhsu supra Sarypul, 04 VII 1897, Lipsky 96" (syntype LE! [LE00051646]); "Seravschan region, inter Guzar et Kshtut, 18 VI 1882, 3-4000', A. Regel" (syntypes LE! [ LE00051647, LE00051653]); "Kshtut, 5-7000', 19 VI 1882, A. Regel" (syntype LE! [LE00051654]); Fl. Jagnob, Uovon, 10 VI 1893, Komarov" (syntypes LE! [LE00051651, LE00051655]); UZBEKISTAN. "Shakhrisjabs, Khtai, 14 VI 1896, Lipsky" (syntypes LE! [LE00051639, LE00051640]); "Denau, Sangardak, 20 1896, Lipsky" (syntype LE! [LE00051645]); "Shakhimardan, 07 VII 1871, O. A. Fedtschenko" (syntype LE); KYRGYZSTAN. "Alabuga in fl. Naryn, 7-8000', 04 VI 1880, A. Regel" (syntypes LE! [LE00051656, LE00051657]); “inter Popak et Osh, 30 VII 1871, A. Regel" (syntype LE). KAZAKHSTAN. "Semirechje, Vernyi-AlexeevkaTurgen, Sorokin" (syntype LE! [LE00051652]); "inter Sjugaty et Kurdai, 24 VI 1871, A. Regel" (syntype LE! [LE00051648]); ibid. 24 VI 1879, A. Regel (syntype LE! [LE00051648] "Karatau, Turchi, 1876, A. Regel" (syntype LE); "Vallis fl. Tschu, Kurdai, 23 VII 1880, A. Regel" (syntype LE! [LE00051643, LE00051650]); "Mt. Alexandrovsky, Aishmara. 1876, A. Regel" (syntype LE).

Distribution in MA \& K: Kazakhstan (S: S Kazakhstan prov., Zhambyl prov.; SE: Almaty prov.), Kyrgyzstan (Batken prov., Ysyk-köl prov., Osh prov., Talas prov., Chüh prov.), Tajikistan (Kŭhistani Badakhshan, Sughd prov., Khatlon prov., Dushanbe prov.), Uzbekistan (Navoi prov., Dzhizak prov., Samarqand prov., Qashqadarya prov., Surxondarya prov., Toshkent prov.), Turkmenistan (Lebap prov.).

Distribution in other Asian countries: Afghanistan.

Distribution area: South-Western Asia.

Galagania gracilis (Kamelin et Pimenov) Kamelin et Pimenov, 1981, in S. K. Cherepanov, Sosud. Rast. SSSR: 21. 1981.

$\equiv$ Elaeosticta gracilis Kamelin et Pimenov, 1978, Novit. Syst. P1. Vasc. 15: 200.

Type: TAJIKISTAN. "Tadshikistania, jugum Karateginum, ad fonte fl. Iljak prope pagum Kaftagysar, 30 VII 1969, Pimenov 1586" (holotype LE!).

Distribution in MA \& K: Tajikistan (Khatlon prov., Dushanbe prov.).

Distribution in other Asian countries: Afghanistan.
Distribution area: South-Western Asia.

Galagania margiana Pimenov et M. G. Vassiljeva, 1983, in Vvedensky (ed.), Opred. Rast. Sred. Azii 7: 374, 223.

Type: TURKMENISTAN. "Turcomania, Badhyz, prope opp. Kuschka, pag. Poltavskoe, 06 VI 1978, Pimenov \& al. 279" (holotype LE!: isotypes BM! [BM000944764], MHA!, MW! [MW0593831]).

Distribution in MA \& K: Turkmenistan (Akhal prov., Mary prov.). Endemic.

Distribution area: South-Western Asia.

Galagania neglecta M. G. Vassiljeva et Kljuykov, 1983, Novit. Syst. Pl. Vasc. 20: 156; 1983, in Vvedensky (ed.), Opred. Rast. Sred. Azii 7: $373,222$.

Type: UZBEKISTAN. "Uzbekistania, Samarkand prov., jugum Seravschanicum, declivum australe prope trajectum Takhta-karacha (AmanKutan), 31 V 1976, Pimenov \& al. 290" (holotype LE!; isotypes BM!, MHA!, MW! [MW0593840]).

Distribution in MA \& K: Uzbekistan (Qashqadarya prov., Samarqand prov., Surxondarya prov.). Endemic.

Distribution area: South-Western Asia.

Galagania platypoda (Aitch. et Hemsl.) M. G. Vassiljeva et Pimenov, 1983, Novit. Syst. Pl. Vasc. 20: 156; 1983, in Vvedensky (ed.), Opred. Rast. Sred. Azii 7: 223.

$\equiv$ Johrenia platypoda Aitch. et Hemsl., 1888, Trans. Linn. Soc. Bot. London (2 ser.) 3: N 38, fig. 29.

Type: AFGHANISTAN. "Afghanistan, Harirud valley, 08 VI 1885, Aichison 620" (lectotype K! [K000695719]; designated here by Pimenov; isolectotypes G! [G00366888], GH [GH00076727], K [K000681158, K000695719], LE!, PH [PH00016525]).

= Peucedanum tenuisectum Regel et Schmalh. subsp. microcarpum Korovin, 1924, Byull. SredneAsiatsk. Gosud. Univ. 7, Suppl. (Sched. Herb. Fl. As. Med. 1-2): 28, in nota.

$=$ Korovinia microcarpa (Korovin) Korovin, 1950, in Nikitin (ed.), Fl. Turkmen. 5: 229; ejusd., 1951, in Schisckkin (ed.), Fl. URSS 17: 213

Type: TURKMENISTAN. "Turkmenia, Kopet-Dagh, 10 stadia rossica S de Firjuza, angustis Schu-sai, zona verticallis stepposa. 30 VI 1923, Korovin" (lectotype TASH! [TASH002560]; designated here by Pimenov; isolectotype TASH [TASH002561]). 
- Korovinia tenuisecta auct. non Nevski et Vved.: Mozaff., 1983, Fam. Umbell. Iran: 122, tab. 318.

Distribution in MA \& K: Turkmenistan (Balkan prov., Akhal prov., Mary prov.).

Distribution in other Asian countries: Afghanistan.

Distribution area: South-Western Asia.

Galagania tenuisecta (Regel et Schmalh.) M. G. Vassiljeva \& Pimenov, 1983, in Vvedensky (ed.), Opred. Rast. Sred. Azii 7: 223.

$\equiv$ Peucedanum tenuisectum Regel et Schmalh., 1882, Izv. Obshch. Ljubit. Estestv. 34(2) (Descr. P1. Nov. Rar. Fedtsch.): 37.

三 Korovinia tenuisecta (Regel et Schmalh.) Nevski \& Vved., 1937, Trudy Bot. Inst. Akad. Nauk SSSR (ser. 1 Fl. Sist. Vyssh. Rast.) 4: 273.

194.

EEulophus tenuisectus (Regel et Schmalh.) M. Hiroe, 1979, Umbelliferae World: 1603.

Type: KAZAKHSTAN. In Turkestaniae montibus karatavicis prope Boroldai, $03 \mathrm{VI}$ 1876, A. Regel" (lectotype LE!, designated by A. M. Geldykhanov 1992: 117); isolectotypes G! [G00366891], K! [K000695718], P! [P02272044]).

Distribution in MA \& K: Kazakhstan (Central: Kzyl-Orda prov.; S: S Kazakhstan prov., Zhambyl prov.), Kyrgyzstan (Batken prov., Talas prov.), Tajikistan (Sughd prov.), Uzbekistan (Buxoro prov., Dzhizak prov., Samarqand prov., Qashqadarya prov., Surxondarya prov., Sirdarya prov., Toshkent prov.), Turkmenistan (Lebap prov.).

Distribution in other Asian countries: Afghanistan.

Distribution area: South-Western Asia.

Helosciadium nodiflorum (L.) W. D. J. Koch,1824, Nova Acta Phys.-Med. Acad. Caes. Leop.-Carol. Nat. Cur. 12(1): 126, fig. 74.

ESium nodiflorum L., 1753, Sp. P1.: 251 .

$\equiv$ Seseli nodiflorum (L.) Scop.,1771, Fl. Carniol. (ed. 2) 1:213.

三Sison nodiflorum (L.) Brot.,1804, Fl. Lusit. 1: 423.

三Apium nodiflorum (L.) Lag., 1821, Amen. Nat. Españ.: 101.

$\equiv$ Helodium nodiflorum (L.) Dumort., 1827, F1. Belg. (Dumortier): 77.

三Lavera nodiflora (L.) Raf.,1840, Good book: 50. 1840.

三Selinum nodiflorum (L.) E. H. L. Krause, 1904, in Deutschl. Fl. (Sturm), ed. 2 12: 34. 1904.
Type: EUROPE. In Europa ad ripas fluviorum [cultivated plants from the garden of George Clifford], Herb. Clifford, 98, Sium 3 (lectotype BMCliff [BM000558307], designated by Jafri in Jafri \& El-Gadi, 1985: 78).

Distribution in MA \& K: Kazakhstan (S: S Kazakhstan prov.), Kyrgyzstan (Batken prov., Jalalabad prov., Osh prov.), Tajikistan (Kŭhistani Badakhshan, Sughd prov., Khatlon prov., Dushanbe prov.), Uzbekistan (Buxoro prov., Navoi prov. [?], Dzhizak prov., Samarqand prov., Qashqadarya prov., Surxondarya prov., Toshkent prov., Fergona prov.), Turkmenistan (Balkan prov., Lebap prov.).

Distribution in other Asian countries: Pakistan, Afghanistan, Iran, Azerbaidzhan, Turkey, Iraq, Saudi Arabia, Yemen, Lebanon, Syria, Israel, Jordan, Cyprus.

Distribution area: Asia (SW, Mediterranean \& W Tropical), Europa, Africa (N), N. America (adv.), S. America (adv.), Australia \& Oceania (adv.).

Heracleum dissectum Ledeb., 1829, Fl. Altaic. (Ledebour) 1: 301. 1829.

EPastinaca dissecta (Ledeb.) Koso-Pol., 1916, Bull. Soc. Imp. Naturalistes Moscou, n. s. 29: 113, nom. illeg. (Art. 53.1), non Vent. (1803).

$\equiv$ Heracleum sphondylium L. f. dissectum (Ledeb.) H. Ohba, 1994, J. Jap. Bot. 69(2): 119.

Type: RUSSIA/KAZAKHSTAN. "In pratis prope pagum Tschetschulicha, metallifodinam Riddersk et alibi [Prope Tschetschulicha], Ledebour 580. 242" (lectotype LE, designated by V. M. Vinogradova 2005: 962; isolectotype P! [P02272123]).

= Heracleum barbatum Ledeb., 1829, Fl. Altaic. (Ledebour) 1: 300.

= Pastinaca barbata (Ledeb.) Koso-Pol., 1916, Bull. Soc. Imp. Naturalistes Moscou, n. s. 29: 113.

= Heracleum dissectum Ledeb. var. barbatum (Ledeb.) Krylov, 1904, F1. Alt. Tomsk. Gub. 3: 534.

Type: KAZAKHSTAN. "[Altai] In pratis montanis [Riv. Pichtovka prope Buchtarminsk 20 VI 1826, Meyer 295" (lectotype LE, designated by V. M. Vinogradova 2005: 962).

Distribution in MA \& K: Kazakhstan (NE: E Kazakhstan prov.; Central: Karaganda prov.; SE: Almaty prov.), Kyrgyzstan (Jalal-abad prov., Ysykköl prov., Osh prov., Talas prov., Chüh prov.), TadzhikistanTajikistan (Sughd prov.), Uzbekistan (Toshkent prov.).

Distribution in other Asian countries: Russia, China, Korea, Mongolia. 
Distribution area: Arctic, N, Central, E \& SW Asia.

Heracleum lehmannianum Bunge, 1849, Delect. Sem. Horti Dorpat. App. 1850: unpaged [2]; id., 1852, Beitr. Fl. Russl. (Lehm. Rel. Bot.): 311.

$\equiv$ Pastinaca lehmanniana (Bunge) Koso-Pol., 1916, Bull. Soc. Imp. Naturalistes Moscou, n. s. 29: 113. 1916.

Type: UZBEKISTAN. "Ad torrentes prope Samarkand, 14 IX 1841, Lehmann" (lectotype P! [P00731531, P00731532, P00731533]; designated here by Pimenov; isolectotype G-BOIS! (sem.)).

= Heracleum afghanicum Kitam., 1958, Acta Phytotax. Geobot. 17: 131. 1958.

Type: AFGHANISTAN. "Near Schibar Pass, 2500 m, 24 VI 1955, Kitamura" (holotype KYO!).

- Heracleum leucocarpum auct. non Aitch. \& Hemsl.: Rech. f.., 1963, in Biol. Skr. 13, 4 (Symb. Afghan. 5): 83. 1963.

Distribution in MA \& K: Kazakhstan (S: S Kazakhstan prov.), Kyrgyzstan (Bakten prov., Jalal-abad prov., Osh prov.), Tajikistan (Kŭhistani Badakhshan, Sughd prov., Khatlon prov., Dushanbe prov.), Uzbekistan (Dzhizak prov., Qashqadarya prov., Samarqand prov., Surxondarya prov., Toshkent prov., Namangan prov. Fergona prov.).

Distribution in other Asian countries: Pakistan, Afghanistan

Distribution area: South-Western Asia.

Heracleum sibiricum L., 1753, Sp. P1., 249. 1753.

$\equiv$ Heracleum sphondylium L. subsp. sibiricum

(L.) Simonk., 1886, Enum. Fl. Transsilv.: 226. 1886.

$\equiv$ Heracleum sphondylium L. var. sibiricum (L.)

Schmalh., 1895, Fl. Sredn. Jushn. Rossii 1: 413. 1895.

$\equiv$ Selinum casparyi E. H. L. Krause, 1904 in Deutschl. Fl. (Sturm), ed. 2 12: 137. 1904.

$\equiv$ Pastinaca sibirica (L.) Calest., 1905 Webbia 1: 244.1905.

Type: RUSSIA. In Sibiria, 352.5 (lectotype LINN [LINN-HL352-5], designated by Reduron in Jonsell \& Jarvis 2002: 84).

$=$ Heracleum flavescens Willd., 1788, Sp. Pl. ed. 4 (Willdenow) 1(2): 1421. 1798.

Type: EUROPE. in Austria.

Distribution in MA \& K: Kazakhstan (W: W Kazakhstan prov., Aktobe prov.; N: Kostonai prov., Akmola prov., N Kazakhstan prov.; NE: E Kazakhstan prov.; Central: Karagandy prov.).

Distribution in other Asian countries: Russia, Mongolia
Distribution area: Asia ( $\mathrm{N} \&$ Central), Europa, N. America (adv.).

Hyalolaena bupleuroides (Schrenk ex Fisch. \& C. A. Mey.) Pimenov \& Kljuykov, 1982, Bot. Zhurn. (Leningrad) 67(7): 887, fig. 9, map 1, 2.

$\equiv$ Carum bupleuroides Schrenk ex Fisch. \& C. A. Mey., 1845, Bull. Phys.-Math. Acad. Petersb. 3: 305.

$\equiv$ Hymenolyma bupleuroides (Schrenk ex Fisch. \& C. A. Mey.) Korovin, 1948, Not. Syst. Herb. Inst. Bot. \& Zool. Acad. Sci. Uzbekistan 12: 51.

Type: KAZAKHSTAN. "Songaria, am Balchasch, 16 VI 1843, Schrenk 357" (lectotype LE!; designated by V. M. Vinogradova, 1999: 840; isotype MANCH!).

Distribution in other Asian countries: China.

Distribution area: Central \& South-Western Asia.

Hyalolaena depauperata Korovin, 1948, Not. Syst. Herb. Inst. Bot. \& Zool. Acad. Sci. Uzbekistan 12: 20.

三Selinum depauperatum (Korovin) M. Hiroe, 1979, Umbelliferae World: 1311.

Type: UZBEKISTAN. "Prov. Kashka-Darja, in montibus humilioribus gypsaceis septentriones versus a urbem Karshi, 31 V 1978, Pimenov \& Kljuykov 232" (neotype LE!, designated by Pimenov \& Kljuykov 1982: 886); isoneotypes MW! [MW0593910, MW0593911]).

Distribution in MA \& K: Uzbekistan (Buxoro prov., Qashqadarya prov., Samarqand prov., Surxondarya prov.). Endemic.

Distribution area: South-Western Asia.

Hyalolaena intermedia Pimenov \& Kljuykov, 1982, Bot. Zhurn. (Leningrad) 67(7): 887, fig. 8, map 1,4 .

Type: KYRGYZSTAN. "Thian-Schan, declivum meridionale jugi Tschatkalensis, in cursu inferiore fluminis Kassansai, supra pag. Kysyltokoi, in arenis rubris, 15 VI 1976, Pimenov, Kljuykov \& Vassiljeva 654" (holotype LE!; isotypes MHA!, MW! [MW0593912]).

Distribution in MA \& K: Kyrgyzstan (Jalalabad prov.), Uzbekistan (Namangan prov., Andijon prov.). Endemic.

Distribution area: South-Western Asia.

Hyalolaena issykkulensis Pimenov \& Kljuykov, 1990, Bot. Zhurn. (Leningrad) 75(1): 92, fig. 3, 4.

Type: KYRGYZSTAN. "Kyrgyzstan, in declivi boreali jugi Terskei Alatau, in valle fl. Chon-Kysylsu, 
14 VII 1974, Pimenov \& Kljuykov 907" (holotype LE!; isotypes MW! [MW0593914, MW0593915]).

Distribution in MA \& K: Kyrgyzstan (Ysykköl prov.).

Distribution in other Asian countries: China.

Distribution area: Central Asia.

Hyalolaena jaxartica Bunge, 1852, Beitr. Fl. Russl. (Lehm. Rel. Bot.): 128; 1854, Mém. Acad. Imp. Sci. Saint-Petersbourg Divers Savants 7: 304.

Type: KAZAKHSTAN. "In der Lehmsteppe am Ssyr-Darja (Jaxartes). [In deserto limoso versus Jaxartam], 09 V 1842, Lehmann" (lectotype LE!; designated here by Pimenov; isolectotypes G [G00366949], P! [P03237360]).

= Holopleura carioides Regel et Schmalh., 1882, Izv. Obshch. Ljubit. Estestv. 34(2) (Descr. Pl. Nov. Rar. Fedtsch.): 28.

Type: UZBEKISTAN. "In Turkestania prope Tschardara in collibus arenosis, in deserto Karak et Kisil-kum, 27 IV 1871, O. A. Fedtschenko" (lectotype LE!, designated by V. M. Vinogradova 2001: 52); Inter Karak-Ati et Adam Kir ullam prope Sultan-bibi in collibus arenosis, Korolkov (syntype LE?).

= Hyalolaena depauperata Korovin, 1948, Not. Syst. Herb. Inst. Bot. \& Zool. Acad. Sci. Uzbekistan 12: 20.

$\equiv$ Selinum depauperatum (Korovin) M. Hiroe, 1979, Umbelliferae World: 1311.

Type: UZBEKISTAN. "Prov. Kashka-Darja, in montibus humilioribus gypsaceis septentriones versus a urbem Karshi, 31 V 1978, Pimenov \& Kljuykov 232" (neotype LE!, designated by Pimenov \& Kljuykov 1982: 886); isoneotypes MW! [MW0593910, MW0593911]).

= Hyalolaena praemontana Korovin, 1962, Trudy Inst. Bot. Akad. Nauk Kazakhsk. SSR 13: 246.

Type: KAZAKHSTAN. "Desertum Kyzylkum, montes Karaktau, in regione Schardaradala [ad margines arenosis Kyzyl-kum, ad pedes montium Karaktau, NW locis Schardaradala] 11 VI 1959, Bykov \& Godvinski" (holotype AA!).

Distribution in MA \& K: Kazakhstan (Central: Karagandy prov., Kzyl-Orda prov.; S: S Kazakhstan prov., Zhambyl prov.), Kyrgyzstan (Batken prov.), Tajikistan (Sughd prov.), Uzbekistan (Kara-kalpak, Buxoro prov., Dzhizak prov., Navoi prov., Qashqadarya prov., Samarqand prov., Surxondarya prov.).

Distribution in other Asian countries: Afghanistan.
Distribution area: Central \& South-Western Asia.

Hyalolaena lipskyi (Korovin) Pimenov \& Kljuykov, 1982, Bot. Zhurn. (Leningrad) 67(7): 888, fig. 10, 11.

三Scaligeria lipskyi Korovin, 1928, Byull. Sredne-Asiatsk. Gosud. Univ. (ser. 8b, Bot.) 2: 34.

$\equiv$ Elaeosticta lipskyi (Korovin) Kljuykov, Pimenov \& V. N. Tikhom., 1976, Byull. Moskovsk. Obshch. Isp. Prir. Otd. Biol. 81(6): 93.

Type: TURKMENISTAN. "Pul-i Chatum, in montibus, 02 V 1885, Korshinsky" (lectotype LE!, designated by V. M. Vinogradova, 1997б: 57).

= Muretia oeroilanica Korovin, 1950, in Schischkin (ed.), Fl. URSS 16: 598, 318. 1950.

= Bunium oeroilanicum (Korovin) M. Hiroe, 1979, Umbelliferae World: 683. 1979.

Type: TURKMENISTAN. "Badghyz, in arenis gypsaceis in zona semideserti montani prope Erojlan-duz, 15 V 1925, Korovin 340" (lectotype TASH! [TASH002305]; designated here by Pimenov; isolectotypes Korovin 351, 352 (LE!, TASH [TASH002304, TASH002306]).

Distribution in MA \& K: Turkmenistan (Akhal prov., Mary prov.).

Distribution in other Asian countries: Afghanistan, Iran

Distribution area: South-Western Asia.

Hyalolaena melanorhiza Pimenov \& Kljuykov, 1983, Bot. Zhurn. (Leningrad) 68(10): 1415, fig. 1. 1983, "melanorrhiza".

Type: TAJIKISTAN. "Badachschan, jugum Jazgulem, in valle fluvii Pjandsh, inter pagum Shipad et fontem Schisgu, in declivi argilloso septentrionali, 30 VII 1981, Pimenov, Kljuykov, Baranova \& Vasilieva 1003, 1004" (holotype LE!; isotypes B! [B 10 0367178], E! [E00002350], JE [JE00025598], MHA!, MW! [MW0593916], TASH! [TASH002351]).

Distribution in MA \& K: Tajikistan (Kühistani Badakhshan). Endemic. The species was also recorded (Breckle et al., 2013: 134) for Afghanistan (N: Baghlan)

Distribution area: South-Western Asia.

Hyalolaena transcaspica (Korovin) Pimenov \& Kljuykov,1982. Bot. Zhurn. (Leningrad) 67(7): 888, fig. 11. 1982.

$\equiv$ Muretia transcaspica Korovin, 1950, in Schischkin (ed.), Fl. URSS 16: 598, 417. 1950. 
$\equiv$ Bunium transcaspicum (Korovin) M. Hiroe, 1979, Umbelliferae World: 683. 1979.

Type: TURKMENISTAN. "Turkmen prov., Kopet Dagh, SSW versus a Ashkhabad, 24 VI 1921, Popov 749, 750" (lectotype TASH! [TASH002301], isolectotype TASH [TASH002302]).

Distribution in MA \& K: Turkmenistan (Balkan prov., Akhal prov.). Endemic.

Distribution area: South-Western Asia.

Hyalolaena trichophylla (Schrenk) Pimenov \& Kljuykov, 1982, Bot. Zhurn. (Leningrad) 67(7): 887, fig. 8, map 1, 1. 1982.

$\equiv$ Carum trichophyllum Schrenk, 1841, Enum. P1. Nov. 1: 61. 1841.

$\equiv$ Conopodium trichophyllum (Schrenk) Korovin, 1924, Byull. Sredne-Asiatsk. Gosud. Univ. 7, Suppl. (Sched. Herb. Fl. As. Med. 1-2): 25. 1924.

$\equiv$ Bunium trichophyllum (Schrenk) H. Wolff, 1927, in Pflanzenr. (Engler) Umbellif.-Apioid.-Ammin. 90(IV, 228): 210. 1927.

$\equiv$ Hymenolyma trichophyllum (Schrenk) Korovin, 1948, Not. Syst. Herb. Inst. Bot. \& Zool. Acad. Sci. 2002b: Uzbekistan 12: 31. 1948.

Type: KAZAKHSTAN. "In desertis versus fl. Aksu [Songaria, Aksu], 10.06 VI.1840, Schrenk" (lectotype LE!, designated by V. M. Vinogradova 1997a: 99; isolectotype P! [P03237366]).

= Seseli scariosum Kar. \& Kir., 1842, Bull. Soc. Imp. Naturalistes Moscou 15: 361. 1842.

= Hymenolyma scariosum (Kar. \& Kir.) Korovin, 1948, Not. Syst. Herb. Inst. Bot. \& Zool. Acad. Sci. Uzbekistan 12: 31. 1948.

Type: KAZAKHSTAN. "In argillosis Songoriae ad rivulum Ai, 1841, Karelin \& Kirilov 1526" (syntypes LE!, MW! [MW0593886], P [P02496402], TK); "fl. Lepsa circa radicem montium Alatau, 06 VI 1841, Karelin \& Kirilov 1526" (lectotype LE!, designated by I. A. Gubanov \& al., 1998: 40).

$=$ Korovinia goloskokovii Bajtenov, Bot. Mater. Gerb. Inst. Bot. Akad. Nauk Kazakhsk. SSR 10: 37. 1977.

Type: KAZAKHSTAN. "Regio ripae dextrae adjacens fl. Ili inter Panfinov and Chorgos, in demissis loessaceis ad marginem arenis Muyunkum, 04 VII 1971, Goloskokov" (holotype AA!; isotypes AA!, LE!).

Distribution in MA \& K: Kazakhstan $(\mathrm{N}$ : Akmola prov.; NE: E Kazakhstan prov.; Central: Karaganda prov.; S: S Kazakhstan prov., Zhambyl prov.; SE: Almaty prov.), Kyrgyzstan (Ysyk-köl prov., Talas prov., Chüh prov.), Turkmenistan (Akhal prov.)
Distribution in other Asian countries: China.

Distribution area: Central \& South-Western Asia.

Hyalolaena tschuiliensis (Pavlov ex Korovin) Pimenov \& Kljuykov, 1982, Bot. Zhurn. (Leningrad) 67(7): 888, fig. 11. 1982.

ESeseli tschuiliense Pavlov ex Korovin, 1962, Trudy Inst. Bot. Akad. Nauk Kazakhsk. SSR 13: 249. 1962.

Type: KAZAKHSTAN. "Montes Tschuilienses, elevatio Chantau, declivia lapidosa, $15 \mathrm{~V}$ 1951, N.V. Pavlov" (holotype AA!; isotype MW! [MW0593890]).

Distribution in MA \& $\mathbf{K}$ : Kazakhstan (S: Zhambyl prov.). Endemic.

Distribution area: Central Asia.

Hymenidium nanum (Rupr.) Pimenov \& Kljuykov, 2000, Feddes Repert. 111(7-8): 549.

$\equiv$ Hymenolaena nana Rupr., 1869, Mém. Acad. Imp. Sci. Saint-Petersbourg (ser. 7) 14(4) (Sert. Tiansch.): 50. 1869.

$\equiv$ Hymenolaena lindleyana Klotzsch var. nana (Rupr.) B. Fedtsch., 1911, Beih. Bot. Centralbl. 28(2): 64. 1911.

Type: KYRGYZSTAN. "Tian-Schan, Taschrobat-Schlucht, 04 VIII 1867, Osten-Saken" (lectotype LE!, designated by V. M. Vinogradova 1997b: 54).

Distribution in MA \& K: Kazakhstan (SE: Almaty prov.), Kyrgyzstan (Ysyk-köl prov., Naryn prov., Osh prov.), Tajikistan (Kŭhistani Badakhshan).

Distribution in other Asian countries: China.

Distribution area: Central Asia

Hymenolaena badachschanica Pissjauk., 1966, Novit. Syst. Pl. Vasc. 3: 186, fig. 1, 2.

Type: TAJIKISTAN. "Asia Media, Pamir occidentalis, Chorog, jugum Schugnanicum, in valle fl. Sangovdara (systema fl. Gunt), 4100 m s. m., in rupibus, 09 VIII 1965, Pissjankova 202" (holotype LE!).

Distribution in MA \& K: Tajikistan (Kŭhistani Badakhshan).

Distribution in other Asian countries: Pakistan, Afghanistan.

Distribution area: Central \& Eastern Asia.

Hymenolaena pimpinellifolia Rupr., 1869, Mém. Acad. Imp. Sci. Saint-Petersbourg (ser. 7) 14(4) (Sert. Tiansch.): 50. 1869. 
$\equiv$ Hymenolaena lindleyana Klotzsch var. pimpinellifolia (Rupr.) B. Fedtsch., 1911, Beih. Bot. Centralbl. 28(2): 64. 1911.

Type: KYRGYZSTAN. "In regione subalpina jugi Tian-Schan. Gebirge welche den Son-kul vom Dschumgal Tunus (Son-kul-Plateau, Santasch), 14 VIII 1867, Osten-Saken" (lectotype LE!, designated by V. M. Vinogradova 1997b: 54).

$=$ Renarda siifolia Regel, 1882, in Trautv. Regel, Maxim. \& Winkl., Decas Pl. Nov.: 5.

Type: KYRGYZSTAN. 'M. Ssussamyr, 12000', 21 VII 1881, Fetissow" (lectotype LE!, designated by V. M. Vinogradova 1997b: 56); isolectotypes BM [BM000944697], BR [BR0000005624362], G!, GH, K! [K000685363, K000685367], P!, S! [S-G5214], US, WU!).

三 Hymenolaena lindleyana Klotzsch var. soongorica Lipsky, 1900, Trudy Imp. S.-Peterburgsk. Bot. Sada 18(1): 71.

Type: KYRGYZSTAN. "Susamyr-tau, 12000', 21 VII 1881, Fetissow" (syntype LE); TAJIKISTAN. "Pamirs, Ljangar-su, 18 VIII 1897, Korshinsky" (syntype LE)

$=$ Hymenolaena lindleyana Klotzsch var. bucharica Lipsky, 1900, Trudy Imp. S.-Peterburgsk. Bot. Sada 18(1): 71.

Type: TAJIKISTAN. "Prope glaciem Dibarar in Karategin, 11000', 11 VIII 1896, Lipsky" (syntype LE); "Pakshif, 7000'-11000', 30 VII 1893, Komarov" (syntype LE); "Zeravschan bassin, Roch, 12 VIII 1893, Komarov" (syntype LE); KYRGYZSTAN. “Arrasan, Mts. Alexandri, 9-11000', 09 VIII 1880, Fetissow" (syntype LE).

Distribution in MA \& K: Kazakhstan ( $\mathrm{S}$ : Zhambyl prov.), Kyrgyzstan (Batken prov., IssykKöl prov., Naryn prov., Osh prov., Talas prov., Chüh prov.), Tajikistan (Sughd prov., Dushanbe prov.). Endemic.

Distribution area: South-Western Asia.

Kadenia dubia (Schkuhr) Lavrova et V. N. Tikhom., 1986, Byull. Moskovsk. Obshch. Isp. Prir. Otd. Biol. 91(2): 93.

$\equiv$ Seseli dubium Schkuhr, 1791, Bot. Handb. 1: 217.

इSelinum pratense Spreng., 1806, Fl. Hal.: 92, tab. 2. 1806.

$\equiv$ Cnidium palustre Rchb. 1832, Fl. Germ. Excurs.: 469 2, nom. illeg. (Art. 52.1).

$\equiv$ Cnidium dubium (Schkuhr) Thell, 1806, In Hegi, 1926, in Ill. Fl. Mittel.-Eur. 5(2): 1305.

三Selinum dubium (Schkuhr) Leute, 1970, Ann. Naturhist. Mus. Wien 74: 501, nom. illeg. Art. 53.1), non Turcz. (1844), nec Krause (1904).
Type: EUROPE, GERMANY. "Wittb. jenseit der Elbe in den querlaufenden Gebüschen unterhalb den Wiesen" (syntypes HAL [HAL0117423, HAL0117424]).

= Seseli venosum Hoffm., 1800, Deutschl. Fl., Jahrgang 3 (Hoffm.): 144.

= Cnidium venosum (Hoffm.) W. D. J. Koch, 1824, Nova Acta Phys.-Med. Acad. Caes. Leop.Carol. Nat. Cur. 12(1): 109.

= Aethusa venosa (Hoffm.) Jess. 1879, Deut. Exkursions-Fl.: 185.

= Meum venosum (Hoffm.) Baill., 1879, Hist. Pl. (Baillon) 7: 191.

$=$ Selinum venosum (Hoffm.) Prantl, 1884, Exkurs.-Fl. Bayern: 284.

= Ligusticum venosum (Hoffm.) Calest. 1905, Webbia 1: 211.

Type: EUROPE. "In pratis sylvaticis trans Albim (? Type B [B-W05929-01 0]).

Distribution in MA \& K: Kazakhstan (W: W Kazakhstan prov., Aktobe prov.; N: Kostonai prov., Akmola prov., N Kazakhstan prov., Pavlodar prov.; NE: E Kazakhstan prov.; Central: Karagandy prov.).

Distribution in other Asian countries: Russia.

Distribution area: Asia ( $\mathrm{N}$ and Central), Europa.

Kafirnifania hissarica (Korovin) Kamelin et Kinzik. 1984, in Ovczinnikov (ed.), Fl. Tadzhikskoi SSR 7: 523, 153, tab. 27, fig. 1-3.

三Peucedanum hissaricum Korovin, 1947, Not. Syst. Herb. Inst. Bot. Zool. Acad. Sci. Uzbekistan 8: 8 .

Type: TAJIKISTAN. "Pamir-Alaj, montes Hissarici, vallis fl. Sarda-i-miona, 26 VI 1930, Pazij \& Mironov 250" (holotype TASH! [TASH002546]; isotype TASH [TASH002547]).

Distribution in MA \& K: Tajikistan (Khatlon d, Dushanbe prov.). Endemic.

Distribution area: South-Western Asia.

Karatavia kultiassovii (Korovin) Pimenov et Lavrova, 1987, Bot. Zhurn. (Leningrad) 72(1): 36.

$\equiv$ Selinum kultiassovii Korovin, 1950, in Schischkin (ed.), Fl. URSS 16: 604, 561.

$\equiv$ Sphaenolobium kultiassovii (Korovin) Pimenov, 1975, Novit. Syst. Pl. Vasc. 12: 245.

Type: KAZAKHSTAN. "Asia Media, montes Talasski Alatau, in pratis udis ad ripem fl. Dzhabogly-su, alt. ca. $2200 \mathrm{~m}, 13$ VIII 1931, N. V. Pavlov 1053" (holotype LE! [LE01065761]; isotype MW! [MW0593927]).

Distribution in MA \& K: Kazakhstan (S: S Kazakhstan prov., Zhambyl prov.). Endemic.

Distribution area: South-Western Asia. 
Kitagawia baicalensis (Redow. ex Willd.) Pimenov, 1986, Bot. Zhurn. (Leningrad) 71(7): 944. $\equiv$ Selinum baicalense Redow. ex Willd. 1809, Enum. Pl. (Willdenow) 1: 306.

$\equiv$ Peucedanum baicalense (Redow. ex Willd.) W. D. J. Koch, 1824, Acta Phys.-Med. Acad. Caes. Leop.-Carol. Nat. Cur. 12(1): 94.

$\equiv$ Pteroselinum sibiricum Rchb. 1832, Fl. Germ. Excurs.: 454.

Type: RUSSIA. "Culta in H. B. Berol. [Origin: ad lacum Baical. Redowsky [5719]" (holotype B-Willd! [B-W 05719-01 0]).

= Selinum obscurum Fisch. ex Trev., 1821, Index Sem. Horti Wratisl. App. 3: 3.

= Peucedanum polyphyllum Ledeb., 1829, Fl. Altaic. (Ledebour) 1: 314.

$=$ Peucedanum polyphyllum Ledeb. var. pubescens Ledeb., 1829, Fl. Altaic. (Ledebour) 1: 315.

Type: RUSSIA "Altai. In rupestribus siccis ad fl. Tscharysch et Kerlyk. [In rupestribus ad fl. Tscharysch], 1826, Ledebour [Bunge] 848" (lectotype LE!, designated by V. M. Vinogradova 2005: 964; isolectotypes CGE!, HAL [HAL0098500, HAL00985501], P [P00731609, P03223233, P03223234, P03223236, P03223238, P03223240]); KAZAKHSTAN “prope Riddersk, Loktewsk et alibi (Ledebour); in collibus arenosis circa Schubinsk ad fl. Irtysch (Meyer)".

= Peucedanum polyphyllum Ledeb. var. glabrum Ledeb., 1829, Fl. Altaic. (Ledebour) 1: 315.

Type: KAZAKHSTAN/RUSSIA. "In sabulosis prope Schubinsk (Meyer) et Loktewsk (Ledebour). [In collibus arenosis pinetis inter redout Schubinsk et Oserki non procul a fortalintis Semipalatinsk. Meyer (lectotype LE!); designated by V. M. Vinogradova (2005: 964); isolectotypes CGE!, HAL [HAL0098500, HAL0098501], P [P00731609, P00731610, P03223234, P03223236, P03223238, P03223240]; RUSSIA. "Prope Loktevsk, Ledebour 1195" (syntype LE!).

= Peucedanum polyphyllum Ledeb. var. lineare Ledeb., 1829, Fl. Altaic. (Ledebour) 1: 315.

Type: KAZAKHSTAN. "In rupestribus siccis prope Riddersk, Ledebour" (holotype LE!).

= Peucedanum caespitosum H. Wolff, 1926, Acta Horti Gothob. 2: 323.

Type: CHINA. "Chili: Hsiao-wu-tai-shan, Tienlin-ssu, Uferabhang. Ad rivulum in declivitate aprica, lapidosa; strandbrink, 23 IX 1921, H. Smith 66" (lectotype UPS!; designated by Pimenov, 2017, in Turczaninowia 20, 2: 166; isolectotype GB! [GB0048816]).
= Peucedanum trinioides H. Wolff, 1926, Acta Horti Gothob. 2: 325.

Type: CHINA. "Chili: Hsiao-wu-tai-shan, Yangkia-p'ing, Tung-lin. auf Gebirgstriften, 1700-1900 m, 27 VIII 1921, H. Smith 452" (syntypes BM [BM000885398], GB! [GB-0048818], UPS!); "Chili: zwischen Tao-lai-shui und Kuo-chewai, 1300-1600 m. 15 IX 1921. H. Smith 364" (lectotype UPS!; designated by Pimenov, 2017, in Turczaninowia 20, 2: 166).

Distribution in MA \& K: Kazakhstan (NE: E Kazakhstan prov.).

Distribution in other Asian countries: Russia, China, Mongolia.

Distribution area: Northern, Central and Eastern Asia.

Komarovia anisosperma Korovin, 1939, in Commem. Vol. 70th Ann. V. L. Komarov: 430.

Type: UZBEKISTAN. "Pamiro-Alaj occidentalis, Mont. Seravschanici prope pag. Urgut, Mt. Chakhmalyk, in angustiis Aine-kor, declivum occidentale, in schistosis, 27 VI 1927, Gnezdillo 259" (holotype TASH! [TASH002522TASH002524, TASH002526].

Distribution in MA \& K: Tajikistan (Sughd prov.), Uzbekistan (Samarqand prov.). Endemic.

Distribution area: South-Western Asia.

Korshinskia bupleuroides Korovin, 1924, Bot. Mater. Gerb. Glavn. Bot. Sada R.S.F.S.R. 5(6): 83.

Type: TAJIKISTAN. "Distr. Chodzhent, montes Mogoltau, Mt. Spa, in calcareis, 24 VI 1923, Popov et Vvedensky 274, 275" (lectotype TASH [TASH002298]; designated by Korovin (1950: 420); isolectotypes LE! [LE00051662], TASH! [TASH002299]).

Distribution in MA \& K: Kazakhstan (S: S Kazakhstan prov.), Tajikistan (Sughd prov.). Endemic.

Distribution area: South-Western Asia.

Note: Schischkin (1950: 414) erroneously placed the name of this species in synonymy Hymenolyma bupleuroides, while in the same edition it is correctly considered as the name of an independent species of Korshinskia (Korovin, 1950: 420).

Korshinskia kopetdaghensis (Korovin) Pimenov et Kljuykov, 1981, Bot. Zhurn. (Leningrad) 66(4): 481; id. 1981, in S. K. Cherepanov, Sosud. rast. SSSR: 23.

$\equiv$ Physospermum kopetdaghense Korovin, 1924, Bot. Mater. Gerb. Glavn. Bot. Sada R.S.F.S.R. 5(6): 84. 
$\equiv$ Scaligeria kopetdaghensis (Korovin) Schischk., 1950, in Schischkin (ed.), Fl. URSS 16: 207, tab. 34, fig. 15.

$\equiv$ Kamelinia kopetdaghensis (Korovin) F. O. Khass. et I. I. Maltzev, 1992, Uzb. Biol. Zhurn. 2: 52.

Type: TURKMENISTAN. "Inter Tschuli [Czuli] et Cheirabad, 26 VI 1898, Litvinov 2449" (lectotype LE! [LE00051258], designated by Pimenov et Kljuykov 1981b: 481; isolectotypes AA, LE! [LE00051257, LE00051259]); “Transcaspia: in mont. Kopetdagh in regione subalpina, 7000' [Arwaz, angustiae], 10-11 VI 1917, Korovin" (syntype TASH - not found); "Transcasp. Prov., Distr. Krasnovodsk., vallis fl. Sumbar, in angustiis Ai-Dere, 30 V 1912, Lipsky 3690" (syntypes LE! [LE00051260, LE00051261, LE00051262, LE00051263, LE00051264]); “Transcasp. Distr., Aschabad reg., Mts. Kopetdagh, Kargil-Nuchur, 10 V 1910, Michelson" (syntype LE! [LE00051265]); "Turkmenistan, Kopetdagh, prope Firusa, 23 VI 1921, Popov 606" (syntypes LE! [LE00051266]).

Distribution in MA \& K: Turkmenistan (Balkan prov., Akhal prov.).

Distribution in other Asian countries: Afghanistan.

Distribution area: South-Western Asia.

Korshinskia olgae (Regel et Schmalh.) Lipsky, 1900, Trudy Imp. S.-Peterburgsk. Bot. Sada 18(1): 59.

EPhysospermum olgae Regel et Schmalh., 1882, Izv. Obshch. Ljubit. Estestv. 34(2) (Descr. Pl. Nov. Rar. Fedtsch.): 40.

三 Conopodium olgae (Regel et Schmalh.) KosoPol., 1916, Bull. Soc. Imp. Naturalistes Moscou, n. s. 29: 205.

Type: KYRGYZSTAN/UZBEKISTAN. "In Kokania, in angustis prope Schagimardan, 07 VII 1871, O. A. Fedtschenko" (holotype LE! [LE00051267], designated by A. M. Geldykhanov 1992: 97).

Distribution in MA \& K: Kyrgyzstan (Batken prov., Jalal-Abad prov.), Tajikistan (Kŭhistani Badakhshan, Sughd prov., Khatlon prov., Dushanbe prov.), Uzbekistan (Navoi prov., Dzhizak prov., Samarqand prov., Qashqadarya prov., Surxondarya prov.), Turkmenistan (Lebap prov.). Endemic.

Distribution area: South-Western Asia.

Korshinskia tianschanica (F. O. Khass. et I. I. Maltzev) Pimenov, 2020, comb. nov.

$=$ Kamelinia tianschanica F. O. Khass. et I. I. Maltzev, 1992, Uzb. Biol. Zhurn. 2: 51.
Type: UZBEKISTAN. "Montes Chatkalenses, montes Chiltenboa, in lapidosis schistosis, 1900 m s. m., 27 VII 1989, Maltzev" (holotype TASH! [TASH002300]; isotypes K!, LE!).

Distribution in MA \& K: Uzbekistan (Toshkent prov.). Endemic.

Distribution area: South-Western Asia.

Note: The issue of Kamelinia's generic independence remains to be debatable, is Kamelinia independent genus or a part of Korshinskia. There are arguments pro et contra, and, in our opinion, there are more of the latters. The essential difference between Kamelinia s. str. (K. tianschanica, but not $K$. kopetdaghensis) and Korshinskia is only the structure of the underground part of plants, while the independence of Kamelinia is not confirmed by molecular (G.V. Degtjareva, unpublished) and partly by carpological data. At the same time, not only the independence of the species $K$. tianschanica, but also its more or less isolated position among the species of Korshinskia is obvious. Most likely, this species should be distinguished as a monotypic subgenus within Korshinskia. Isotype of the species name must be in LE.

Korshinskia Lipsky subgen. Kamelinia (F. O. Khass. et I. I. Maltzev) Pimenov, stat. nov.

$\equiv$ Kamelinia F. O. Khass. et I. I. Maltzev, 1992, Uzb. Biol. Zhurn. 2: 50. Type: Kamelinia tianschanica F. O. Khass. et I. I. Maltzev, 1992, Uzb. Biol. Zhurn. 2: 51 .

Kozlovia paleacea (Regel et Schmalh.) Lipsky, 1904, Trudy Imp. S.-Peterburgsk. Bot. Sada 23: 147.

三 Albertia paleacea Regel et Schmalh., 1878, Trudy Imp. S.-Peterburgsk. Bot. Sada 5(2): 606.

$\equiv$ Trachydium paleaceum (Regel et Schmalh.) M. Hiroe, 1979, Umbelliferae World: 713.

Type: UZBEKISTAN. "Vallis fl. Zarawschan, inter Jarbash et Djiaman, 1260'-2040', 01 V 1869, O. A. Fedtschenko" (lectotype LE! [LE00051316], designated by A. M. Geldykhanov 1992: 90); “inter Karasu et Katty-kurgan. 26 IV 1869. O. A. Fedtschenko" (syntype LE]).

Distribution in MA \& K: Kyrgyzstan (Batken prov., Jalal-Abad prov.), Tajikistan (Kŭhistani Badakhshan, Sughd prov., Khatlon prov., Dushanbe prov.), Uzbekistan (Navoi prov., Dzhizak prov., Samarqand prov., Qashqadarya prov., Surxondarya prov., Sirdarya prov., Toshkent prov., Namangan prov., Fergona prov.), Turkmenistan (Mary prov.).

Distribution in other Asian countries: Afghanistan.

Distribution area: South-Western Asia. 
Krasnovia longiloba (Kar. et Kir.) Popov, 1950, in Schischkin (ed.), Fl. URSS 16: 118, 592, tab. 15, fig. 1.

$\equiv$ Sphallerocarpus longilobus Kar. et Kir., 1841, Bull. Soc. Imp. Naturalistes Moscou 14: 432.

$\equiv$ Chaerophyllum sphallerocarpus Kar. et Kir., 1842, Bull. Soc. Imp. Naturalistes Moscou 15: 367, nom. illeg. (Art. 52.1).

$\equiv$ Chaerophyllum longilobum (Kar. et Kir.) O. et B. Fedtsch., 1909, Consp. Fl. Turkest. (O. A. Fedtschenko et B. A. Fedtschenko) 3: 120.

$\equiv$ Conopodium longilobum (Kar. et Kir.) KosoPol., 1916, Bull. Soc. Imp. Naturalistes Moscou, n. s. 29: 206.

$\equiv$ Kozlovia longiloba (Kar. et Kir.) Spalik et S. R. Downie, 2001, Ann. Missouri Bot. Gard. 88(2): 289.

Type: KAZAKHSTAN. "In rupestribus montium Tarbagatai ad torrentem Dschanybek, 1840, Karelin \& Kirilov 272" (lectotype LE! [LE0001010], designated by I. A. Gubanov \& al., 1993: 41); isolectotypes LE [LE00051011, LE00051012], NY (sub n 272) [NY00405793], P (sub n 272) [P03212484]).

Distribution in MA \& K: Kazakhstan (NE: E Kazakhstan prov.; S: Zhambyl prov.; SE: Almaty prov.), Kyrgyzstan (Ysyk-köl prov., Chüh prov.).

Distribution in other Asian countries: China.

Distribution area: Central and South-Western Asia.

Kuramosciadium corydalifolium Pimenov, Kljuykov et Tojibaev, 2011, Syst. Bot. 36(2): 492.

Type: UZBEKISTAN. "Uzbekistania, jugum Kurama, Parda Tursun, juxta fontem fluminis Novbulak, in detritis mobilibus, $\mathrm{h}=2700-2900$ m. 20 VII 2009, Tojibaev" (holotype TASH! [TASH002358], isotypes MW! [MW0593920, MW0593921]).

Distribution in MA \& K: Uzbekistan (Namangan prov.). Endemic.

Distribution area: South-Western Asia.

Ladyginia bucharica Lipsky, 1904, Trudy Imp. S.-Peterburgsk. Bot. Sada 23: 150, tab. 9.

$\equiv$ Ferula bucharica (Lipsky) Koso-Pol., 1916, Vestn. Tiflissk. Bot. Sada 11 [1915]: 137, 166.

Type: TAJIKISTAN. "Hissar, Mt. Tach-Bulak, 4-6000', 29-30 IV 1883, A. Regel" (syntype); "Iomut, decl. orient. Gasi-Mailik Mts., inter Vaschch et Kafirnigan, 3000', 13 V 1883, A. Regel" (syntype LE); "Karadagh, 23 VI 893, Lipsky" (syntype LE!); "Baldzhuan, 3000', Mussa" (syntypes LE, LECB [LECB0002308]); "Kurgan-Tube: Sang-Tuda ad fl. Vaschch, 2000-2500', 17 V 1883, A. Regel" (lectotype LE!, designated by A. M. Geldykhanov 1992: 157); "Jakkabag: Jangi-kischlak, 15 VI 1896, Lipsky" (syntype LE); "Bova-schody, 03 VI 1897, Lipsky" (syntype: LE); UZBEKISTAN. "Baisun: Sary-Kamysch, 2800 m, 07 VI 1897, Lipsky 46" (syntype LE!); TAJIKISTAN. "Kabadian: Babatag, ad declivibus, 15 V 1897, Korshinsky" (syntype LE).

Distribution in MA \& K: Tajikistan (Sughd prov., Khatlon prov., Dushanbe prov.), Uzbekistan (Dzhizak prov., Samarqand prov., Qashqadarya prov., Surxondarya prov.), Turkmenistan (Lebap prov.).

Distribution in other Asian countries: Afghanistan.

Distribution area: South-Western Asia.

Ledebouriella multiflora (Ledeb.) H. Wolff, 1910, in Pflanzenr. (Engler) Umbellif.-Apioid.Ammin. 43(IV, 228): 191.

$\equiv$ Rumia multiflora Ledeb., 1844, Fl. Ross. (Ledebour) 2: 281.

Type: KAZAKHSTAN. "[Altai?] In montibus deserti Kirghisici, Sievers, 1623 (10)" (holotype B-Willd; designated by V. M. Vinogradova (2007: 270) as "holotypus"; isolectotype LE! [LE00051461]).

= Stenocoelium tenuifolium Korovin, 1962, Trudy Inst. Bot. Akad. Nauk Kazakh. SSR 13: 256.

Type: KAZAKHSTAN. "Altai, mons Mramornaja, ad declivitatem lepidosam non procul pag. Alexeevka, 17 VII 1958, Stepanova" (lectotype AA!, designated as "typus" by Goloskokov (1963: 29); "Tarbagatai, in angustis riv. Tschitlosty, alt. $1200 \mathrm{~m}$, ad locos lapidosos, 19 VII 1955, Stepanova" (syntype AA!).

Distribution in MA \& K: Kazakhstan (NE: E Kazakhstan prov.).

Distribution in other Asian countries: China.

Distribution area: Central Asia.

Ledebouriella seseloides (Hoffm.) H. Wolff, 1910, in Pflanzenr. (Engler) Umbellif.-Apioid.-Ammin. 43(IV, 228): 192.

$\equiv$ Rumia seseloides Hoffm., 1816, Gen. Pl. Umbell. (ed. 2): 174, p. p.

$\equiv$ Cachrys seseloides (Hoffm.) M. Bieb. 1819, Fl. Taur.-Cauc. 3: 217.

$\equiv$ Trinia seseloides (Hoffm.) Ledeb., 1829, Fl. Altaic. (Ledebour) 1: 357, "seselioides".

$\equiv$ Johrenia seseloides (Hoffm.) Koso-Pol., 1916, Bull. Soc. Imp. Naturalistes Moscou, n. s. 29: 133. 
$\equiv$ Johrenia sieversii Koso-Pol., 1916, Bull. Soc. Imp. Naturalistes Moscou, n. s. 29: 133, nom. illeg. (Art. 52.1).

Type: KAZAKHSTAN. "Altai in campis et collibus siccis prope montes [lapidosis ad montam] Tschingistau, deserti soongoro-kirghisici, VIII 1826, C. A. Meyer 226" (neotype LE! [LE00051463]; designated by M. M. Fedoronchuk 1983: 151; isoneotypes LE [LE00051459, LE00051460, LE00051462, LE00051464], P [P04366556]).

Distribution in MA \& K: Kazakhstan (Central: Karagandy prov.). Endemic.

Distribution area: Central Asia.

Leutea turcomanica (Schischk.) V. M. Vinogr. 2004, Novit. Syst. P1. Vasc. 36: 174.

$\equiv$ Peucedanum involucratum Korovin, 1924, Bot. Mater. Gerb. Glavn. Bot. Sada R.S.F.S.R. 5(6): 84, nom illeg. (Art. 53.1), non W. D. J. Koch (1824).

$\equiv$ Peucedanum turcomanicum Schischk., 1950, Fl. Turkm. 5: 230, tab. 39. 1950, nom. inval.], 1951, in Schischkin (ed.), Fl. USSR 17: 177, tab. 18, fig. 1.

$\equiv$ Ferula turcomanica (Schischk.) Pimenov, 1981, in S. K. Cherepanov, Sosud. Rast. SSSR: 21, nom. inval. (Art. 53.1), non M. Hiroe (1979).

Type: TURKMENISTAN. "Transcaspia in montibus Bolchie Balchany ad rupes in regione subalpina [fontes Bash-Mychur], 12 VII 1923, Korovin 723, 725" (lectotype LE! [LE00052168]; designated by V. M. Vinogradova (2004: 174); isolectotypes LE [LE00052169, LE00052170], TASH! [TASH002548-TASH002551]); "fonts Bash-Mygur. 12 VII 1923. Korovin 723, 724, 728" (syntypes LE!, TASH [TASH002548, TASH002549, TASH002550, TASH002551])

Distribution in MA \& K: Turkmenistan (Balkan prov., Akhal prov.).

Distribution in other Asian countries: Afghanistan, Iran.

Distribution area: South-Western Asia.

Lipskya insignis (Lipsky) Nevski, 1937, Trudy Bot. Inst. Akad. Nauk SSSR (ser. 1 Fl. Sist. Vyssh. Rast.) 4: 272.

三Schrenkia insignis Lipsky, 1900, Trudy Imp. S.-Peterburgsk. Bot. Sada 18(1): 73.

$\equiv$ Anidrum insigne (Lipsky) Koso-Pol., 1920, Izv. Glavn. Bot. Sada R.S.F.S.R. 36, 1 (Fl. As. Ross. 15): 83.

$\equiv$ Hippomarathrum insigne (Lipsky) M. Hiroe, 1979, Umbelliferae World: 1224, "insignis".

Type: TAJIKISTAN. "In prov. Buchara, Jakkabagh in collibus herbidis, Bova-Schody,
3000', 03 VI 1897, Lipsky [264]" (lectotype LE! [LE00051041], designated by V. M. Vinogradova 1997b: 58; isolectotypes LE [LE00051042LE00051048], MPU [MPU020976]).

Distribution in MA \& K: Tajikistan (Sughd prov.), Uzbekistan (Dzhizak prov., Qashqadarya prov., Surxondarya prov.), Turkmenistan (Lebap prov.). Endemic.

Distribution area: South-Western Asia.

Lomatocarpa alata (Korovin) Pimenov et Sennikov, comb. nov.

$\equiv$ Meum alatum Korovin, 1947, Not. Syst. Herb. Inst. Bot. \& Zool. Acad. Sci. Uzbekistan 8: 15; non Baill., 1879, nom. illeg. (Art. 53.1).

$\equiv$ Aulacospermum alatum Korovin, 1963, in N. V. Pavlov, Fl. Kazakhst. 6: 365.

= Lomatocarpa korovinii Pimenov, 1981, in Cherepanov, Sosud. Rast. SSSR: 24, nom. illeg. (Art. 52.1).

Type: UZBEKISTAN. "Tian-Schan occidentalis, vallis fl. Angren, apud put. Arasan, Mts. Aktau, 3100 m”, 01 IX 1938, Pjataeva \& Momotov 1495 (holotype TASH! [TASH002342!).

Distribution in MA \& K: Kazakhstan (S: S Kazakhstan prov.), (Jalal-Abad prov., Talas prov.), Tajikistan (Sughd prov.), Uzbekistan (Toshkent prov.). Endemic.

Distribution area: South-Western Asia.

Note: The nomenclatural details see in A. N. Sennikov \& M. G. Pimenov, Nomenclatural notes on four species of Umbelliferae (Apiaceae) in Middle Asia. Annales Botanicae fennicae (in press)

Lomatocarpa albomarginata (Schrenk) Pimenov et Lavrova, 1987, Bot. Zhurn. (Leningrad) 72(1): 35 .

$\equiv$ Neogaya simplex (L.) Meisn.var. albomarginata Schrenk, 1842, Enum. Pl. Nov. 2: 41.

$\equiv$ Pachypleurum albomarginatum (Schrenk) Rupr., 1869, Mém. Acad. Imp. Sci. Saint-Petersbourg (ser. 7) 14(4) (Sert. Tiansch.): 49.

$\equiv$ Neogaya albomarginata (Schrenk) O. et B. Fedtsch., 1909, Consp. Fl. Turkest. (O. A. Fedtschenko et B. A. Fedtschenko) 3: 97.

$\equiv$ Alposelinum albomarginatum (Schrenk) Pimenov, 1982, Byull. Moskovsk. Obshch. Isp. Prir. Otd. Biol. 87, 1: 113.

Type: KAZAKHSTAN. "Songarei, in montibus Dschabyk, 22 VII 1841, Schrenk 85I" (holotype LE!).

= Ligusticum wolffianum Fedde ex H. Wolff, 1930, Repert. Spec. Nov. Regni Veg. 27: 318. 
Type: AFGHANISTAN. "Pamir [Pamir Boundary Commission], in nullahs alongside running water, 14000-15000 ft.,1895, Alcock 17712" (lectotype BM! [BM000826930]; designated by Pimenov, 2017, in Turczaninowia 20, 2: 173); isolectotype DD!).

= Pachypleurum aemulans Korovin, 1963, in Pavlov (ed.), Fl. Kazakhst. 6: 309, nom. inval. (Art. 39.1), descr. ross.

= Cortia schmidii Nasir, 1972, in Nasir et Ali (eds.), Fl. W Pakistan 20: 115, fig. 34 (A-C).

$=$ Ligusticum schmidii (Nasir) Farille et S. B. Malla, 1985, Candollea 40(2): 551.

Type: PAKISTAN. "Chitral, upper Yarkhun Vy, Schmid 2365" (holotype RAW).

- Neogaya simplex auct. non Meisn.: O. et B. Fedtsch., 1911, Beih. Bot. Centralbl. 28(2): 42.

- Pachypleurum alpinum auct. non Ledeb.: Schischk., 1950, in Schischkin (ed.), Fl. URSS 16: 579 , p. p.

Distribution in MA \& K: Kazakhstan (S: S Kazakhstan prov., Zhambyl prov.; SE: Almaty prov.), Kyrgyzstan (Batken prov., Jalal-Abad prov., Ysyk-köl prov., Naryn prov., Osh prov., Talas prov., Chüh prov.), Tajikistan (Khatlon prov., Dushanbe prov.), Uzbekistan (Qashqadarya prov., Samarqand prov., Toshkent prov.).

Distribution in other Asian countries: China, Afghanistan.

Distribution area: Central \& South-Western Asia.

Lomatocarpa multivittata Pimenov et Kljuykov, 2014, Bot. Zhurn. (St. Petersburg) 99(5): 605, fig. 5,6 .

Type: UZBEKISTAN. "Uzbekistania, jugum Chatkalense, decluvum australe, reservatum montano-sylvaticum Chatkalense, partition Baschkyzylsai, Kurgantasch, in itinere geologicum, declivia schistosa, 2800 m. 18 VIII 1978, Krasovskaya" (holotype MW! [MW0595552]).

Distribution in MA \& K: Uzbekistan (Toshkent prov.). Endemic.

Distribution area: South-Western Asia.

Lomatocarpa steineri (Podlech) Pimenov 1982, Byull. Moskovsk. Obshch. Isp. Prir. Otd. Biol. 87(1): 116.

= Ligusticum steineri Podlech, 1970, Mitt. Bot. Staatsamml. München 8: 173.

Type: AFGHANISTAN. "Nordost-Afghanistan, prov. Kapisa, Oberes Panjir-Tal, Passh'he am Übergang von Kur-Petau in das obere Dekhawak-
Tal. 3800 m, 19 VIII 1965, Podlech 12497" (holotype $\mathrm{M}$ ! [M0172883]; isotypes G! [G00367032], K [K000695867], W! [W 1974-0023251]).

= Pachypleurum linearilobum Korovin, 1973, Izv. Akad. Nauk Tadzh. SSR, Otd. Estest. Nauk 2(51): 9 .

Type: TAJIKISTAN. "Pamir occidentalis, regio Schachdariensis, adversus pago Roschtkaly, in altitudine 3800 m s. m. 29 VII 1960, Agachanajanz 277" (holotype TAD!).

Distribution in MA \& K: Tajikistan (Kŭhistani Badakhshan).

Distribution in other Asian countries: Afghanistan.

Distribution area: South-Western Asia.

Mediasia macrophylla (Regel et Schmalh.) Pimenov, 1974, Novit. Syst. P1. Vasc. 11: 255, fig. 1-3.

三Seseli macrophyllum Regel et Schmalh., 1882, Izv. Obshch. Ljubit. Estestv. 34(2) (Descr. Pl. Nov. Rar. Fedtsch.): 31.

$\equiv$ Athamanta macrophylla (Regel et Schmalh.) Korovin, 1926, Schedae Herb. Fl. As. Med. 10): 10, N 239.

Type: UZBEKISTAN. "In Kokania, in angustiis in vicinitate urbis Schagimardan, 07 VII 1871, O. A. Fedtschenko" (lectotype LE! [LE01065990], designated by A. M. Geldykhanov 1992: 142; isolectotype LE! [LE01065991]); "In Turkestaniae montibus Alatavicis in valle fluvii Tschotkal, 6000' alt., A. Regel" (syntype LE! [LE01065989]); KAZAKHSTAN. "In montibus karatavicis prope Saylik, 3-5000' alt., 1876, A. Regel"' (syntypes K! [K000695779], LE).

Distribution in MA \& K: Kazakhstan (S: S Kazakhstan prov.), Kyrgyzstan (Batken prov., JalalAbad prov., Osh prov., Chüh prov.), Tajikistan (Kŭhistani Badakhshan, Sughd prov., Khatlon prov., Dushanbe prov.), Uzbekistan (Dzhizak prov., Samarqand prov., Qashqadarya prov., Surxondarya prov., Sirdarya prov., Toshkent prov.), Turkmenistan (Lebap prov.).

Distribution in other Asian countries: Afghanistan

Distribution area: South-Western Asia.

Mogoltavia narynensis Pimenov et Kljuykov, 1992, Bot. Zhurn. (St. Petersburg) 77(2): 92, fig. 1.

Type: KYRGYZSTAN. "Asia Media, Kyrgyzstan, Prov. Osch, distr. Toktogul, in systemate fluminis Naryn, prope ostium fluvii Kokomeren, in loco Ak-Tatyr, in partitionibus schistoso-lapidosis, subdesertum artemiseto-salsolacearum, 13 VI 1977, 
Aidarova et al." (holotype MW! [MW0594079]).

Distribution in MA \& K: Kyrgyzstan (Naryn prov.). Endemic.

Distribution area: South-Western Asia.

Mogoltavia sewerzowii (Regel) Korovin, 1947, Not. Syst. Herb. Inst. Bot. \& Zool. Acad. Sci. Uzbekistan 8: 11.

$\equiv$ Carum sewerzowii Regel, 1878, Trudy Imp. S.-Peterburgsk. Bot. Sada 5(2): 587.

$\equiv$ Bunium sewerzowii (Regel) Drude, 1898, in Nat. Pflanzenfam. (Engler \& Prantl) 3(8): 194.

$\equiv$ Peucedanum sewerzowii (Regel) Korovin, 1926, Byull. Sredne-Asiatsk. Gosud. Univ. 14, Suppl. (Sched. Herb. Fl. As. Med. 10): 13, N 245, "severtzovii".

Type: TAJIKISTAN. "Turkestan, Mogoltau. 25 IV-04 V 1877. Sewerzow" (lectotype LE! [LE00051524], designated by V. P. Botschantzev (Vinogradova 1997a: 98); isolectotypes LE! [LE00051525, LE00051526]; Mogoltau. 25 IV 1877. Sewerzow (syntypes LE! [LE00051527, LE00051528]).

= Peucedanum gypsaceum Korovin, 1924, Bot. Mater. Gerb. Glavn. Bot. Sada R.S.F.S.R. 5(6): 75.

Type: KYRGYZSTAN. "Prov. Fergana, prope petroleas "Santo", ad colles gypsiferos, VI 1922. Popov \& Vvedensky" (syntype TASH! [TASH002544]); "Fergana, montes Sary-tau, in planitiae Rabat districti Kokandensis. 13 VI 1920. Popov 523, 526" (lectotype LE! [LE00052121], designated by V. M. Vinogradova (2001: 54); isolectotype TASH! [TASH002543, TASH002545]); TAJIKISTAN. "Prov. Samarqand, distr. Chodshent, in montibus Mogol-tau supra sepulerum Tschaschma-arzanak in rupibus graniticus. $15-23 \mathrm{~V}$ 1924. Popov \& Vvedensky 245" (syntypes TASH! [TASH003712]).

Note: List of herbarium, edited in framework of the HFAM, contains two collections. Syntype P. gypsaceum (Popov et Vvedensky 245) from Mogoltau Mts., is situated in the upper left corner of sheet. Plant, collected by A. I. Vvedensky in distr. Kokand, does not belong to type material of the species.

Distribution in MA \& K: Kyrgyzstan (Batken prov., Osh prov.), Tajikistan (Sughd prov.), Uzbekistan (Namangan prov., Fergona prov.). Endemic.

Distribution area: South-Western Asia.

Myrrhoides nodosa (L.) Cannon, 1968, Feddes Repert. 79(1-2): 65. $\equiv$ Scandix nodosa L., 1753, Sp. Pl.: 257.

$\equiv$ Chaerophyllum nodosum (L.) Crantz, 1767, Cl. umbell. emend.: 76.

$\equiv$ Torilis macrocarpa Gaertn. 1788, Fr. Sem. P1. 1: 183, tab. 20, nom. illeg. (Art. 52.1).

$\equiv$ Torilis tumida Moench,1794, Methodus (Moench): 102, nom. illeg. (Art. 52.1).

$\equiv$ Anthriscus nodosa (L.) Pers. 1805, Syn. P1. 1: 320.

$\equiv$ Anthriscus scandix M. Bieb. 1808, Fl. Taur.Cauc. 1: 231, nom. illeg. (Art. 52.1).

$\equiv$ Biasolettia nodosa (L.) Bertol., 1837, Fl. Ital. (Bertoloni) 3: 191.

$\equiv$ Acularia nodosa (L.) Raf. 1840, Good Book: 53.

$\equiv$ Physocaulis nodosus (L.) W. D. J. Koch, 1843, Syn. Fl. Germ. Helv. (ed. 2): 348.

$\equiv$ Fiebera nodosa (L.) Opiz, 1852, Seznam: 44.

Type: EUROPE, ITALY. "In Sicilia, 364.6" (lectotype LINN [LINN-HL364-6], designated by Hedge et Lamond 1987: 101)

Note: According to another information, the origin of type material is Uppsala (Sweden).

Distribution in MA \& K: Kazakhstan ( $\mathrm{S}$ : S Kazakhstan prov.), Kyrgyzstan (Jalal-Abad prov.), Tajikistan (Khatlon prov., Dushanbe prov.), Uzbekistan (Navoi prov., Samarqand prov., Qashqadarya prov., Toshkent prov.), Turkmenistan (Balkan prov., Akhal prov.).

Distribution in other Asian countries: Russia, Azerbaidzhan, Georgia, Armenia, Turkey, Iraq, Lebanon, Syria, Israel, Jordan.

Distribution area: Asia (Northern, South-Western and Mediterranean), Europa, Africa.

Oedibasis apiculata (Kar. et Kir.) Koso-Pol., 1916, Bull. Soc. Imp. Naturalistes Moscou, n. s. 29: 175.

$\equiv$ Carum apiculatum Kar. et Kir. 1842, Bull. Soc. Imp. Naturalistes Moscou 15: 358.

$\equiv$ Bunium apiculatum (Kar. et Kir.) Drude, 1898, in Nat. Pflanzenfam. (Engler et Prantl) 3(8): 184.

Type: KAZAKHSTAN. "In arenosis inter montis Arganaty et ontem Tschingildy nec non ad fl. Lepsa, VII 1841, Karelin \& Kirilov 360" (lectotype LE! [LE00051477], designated by V. M. Vinogradova 1999: 84; isolectotypes LE! [LE00051478LE00051483], MW!).

= Peucedanum rapiferum Trautv. 1866, Bull. Soc. Imp. Naturalistes Moscou 39(2): 326.

Type: KAZAKHSTAN. "In deserto sabuloso arido fl. Tschu adjacente. 10 VIII 1842. Schrenk" (holotype LE!) 
$=$ Oedibasis apiculata (Kar. et Kir.) Koso-Pol. subsp. australis Kamelin, 1977, Novit. Syst. P1. Vasc. 14: 189.

Type: KAZAKHSTAN. "Kasachstania australis, prope pag. Mankent, 25 V 1916, Lipsky 550" (holotype LE!).

Distribution in MA \& K: Kazakhstan (NE: E Kazakhstan prov.; Central: Karagandy prov., Kzyl-Orda prov.; S: S Kazakhstan prov., Zhambyl prov.; SE: Almaty prov.), Kyrgyzstan (Batken prov., Jalal-Abad prov., Naryn prov., Osh prov., Talas prov., Chüh prov.), Tajikistan (Sughd prov.), Uzbekistan (Dzhizak prov., Navoi prov., Toshkent prov.). Endemic.

Distribution area: Central and South-Western Asia.

Oedibasis chaerophylloides (Regel \& Schmalh.) Korovin, 1926 Byull. Sredne-Aziatsk. Gosud. Univ. 14(Suppl.): 14.

$\equiv$ Carum chaerophylloides Regel \& Schmalh., 1978.Acta Horti Petropolit. 5(2): 587, 1878, non

Carum chaerophylloides Regel \& Schmalh., Acta Horti Petropolit. 5(2): 585. 1878

Type: TAJIKISTAN. "In Kokaniae valle fluvii Sarawschan, in montibus Daschti Kasi, 4000' alt., O. A. Fedtschenko" (holotype LE).

= Carum tamerlanii Lipsky, 1904, Trudy Imp. S.-Peterburgsk. Bot. Sada 23: 129, nom. illeg.

$=$ Oedibasis tamerlanii (Lipsky) Korovin ex Nevski, 1937, Trudy Bot. Inst. Akad. Nauk SSSR (ser. 1. Fl. Sist. Vyssh. Rast. 4: 273.

Type: UZBEKISTAN. "Vallis fl. Zeravschan, montibus prope Dashti-Kasy, $31 \mathrm{~V} 1869$, O. A. Fedtschenko" (lectotype LE! [LE00051593], designated by A. M. Geldykhanov 1992: 118); "Angustis Tamerlan, inter Dzhisak et Yany-kurgan, 19 V 1882, A. Regel" (syntype LE! [LE00051592); "Kum, 03 VI 1893, Komarov" (syntype LE! [LE00051586]); "Urgut, 21 V 1892, Komarov" (syntypes LE! [LE00051584, LE00051585]); "Chakhrisiabz, Chut, 05 VII 1896, Lipsky" (syntype LE! [LE00051587]); "Niaz-bek, prope Tashkent, Lipsky" (syntypes LE! [LE00051588, LE00051589].

Distribution in MA \& K: Kyrgyzstan (Batken prov., Jalal-Abad prov., Osh prov.), Tajikistan (Sughd prov.), Uzbekistan (Buxoro prov., Navoi prov., Dzhizak prov., Samarqand prov., Qashqadarya prov., Surxondarya prov.), Turkmenistan (Lebap prov.). Endemic.

Distribution area: South-Western Asia.

Note: The nomenclatural details see in A. N. Sennikov \& M. G. Pimenov, Nomenclatural notes on four species of Umbelliferae (Apiaceae) in Middle Asia. Annales Botanicae fennicae (in press).

Oedibasis platycarpa (Lipsky) Koso-Pol., 1916, Bull. Soc. Imp. Naturalistes Moscou, n. s. 29: 175.

$\equiv$ Carum platycarpum Lipsky, 1904, Trudy Imp.

S.-Peterburgsk. Bot. Sada 23: 132.

$\equiv$ Bunium platycarpum (Lipsky) H. Wolff, 1927, in Pflanzenr. (Engler), Umbellif.-Apioid.-Ammin. 90(IV, 228): 208.

Type: UZBEKISTAN. "Prov. Syr-Darja, inter Pskem et Nanai, 01 VI 1903, Lipsky 284" (lectotype LE! [LE00051513], designated by V. M. Vinogradova 1997a: 98, isolectotype LE! [LE00051514]); "Tianschan occid., valle fl. Chimgan, 1900, Chomutova" (syntype LE); "Valle fl. Pskem, Obi-Galva. 02 VIII 1902. B. A. Fedtschenko" (syntypes LE [LE00051515, LE00051516]).

= Oedibasis karatavica Korovin, 1951, in Schischkin (ed.), Fl. URSS 17: 355, 207.

= Carum karatavicum (Korovin) M. Hiroe, 1979, Umbelliferae World: 871, "karatavica".

Type: KAZAKHSTAN. "Asia Media. Montes Karatau [Tian-schan occidentalis, montes Karatau, declivum NW schistoso-lapidosum, locum Chuschka-bulak dictum, 23 V 1935, Pjataeva 141" (holotype TASH! [TASH002567]).

Distribution in MA \& K: Kazakhstan (S: S Kazakhstan prov., Zhambyl prov.), Kyrgyzstan (Jalal-Abad prov.), Uzbekistan (Toshkent prov.). Endemic.

Distribution area: South-Western Asia.

Oenanthe aquatica (L.) Poir., 1798, in Encycl. (Lamarck et al.) 4: 530, "aquaticum".

$\equiv$ Phellandrium aquaticum L., 1753, Sp. Pl.: 255.

$\equiv$ Ligusticum phellandrium Crantz 1767, Stirp. Austr. Fasc. 3: 84.

$\equiv$ Oenanthe phellandrium Lam., 1779, Fl. franç. (Lamarck) 3: 432.

$\equiv$ Phellandrium divaricatum S. F. Gray, 1821, Nat. arr. Brit. pl. 2: 508.

$\equiv$ Selinum phellandrium (Crantz) E. H. L. Krause, 1904, in Deutschl. Fl. (Sturm), ed. 2 12: 93, tab. 15.

Type: EUROPA. "In Europae fossis, Herb. Clifford, 100, Phellandrium 1" (lectotype BM-Cliff [BM000558326], designated by Reduron et Jarvis in Jarvis et al., 1993: 75); Leiden, van Royen (syntype L! [L0053017]).

Distribution in MA \& K: Kazakhstan (W: W Kazakhstan prov., Aktobe prov.; N: Kostonai prov., Akmola prov., N Kazakhstan prov.; NE: E Kazakh- 
stan prov.; Central: Karagandy prov.; SE: Almaty prov.).

Distribution in other Asian countries: Russia, Mongolia, Iran, Turkey.

Distribution area: Asia (Northern, Central and South-Western), Europa, N. America (adv.), Australia \& Oceania (adv.).

Oenanthe fedtschenkoana Koso-Pol., 1916, Izv. Imp. S.-Peterburgsk. Bot. Sada 16: 226.

Type: UZBEKISTAN. "Turkestania, Kokan, O. A. Fedtschenko" (holotype MW? - not found).

Distribution in MA \& K: Uzbekistan (Fergona prov.). Endemic.

Distribution area: South-Western Asia.

Oenanthe silaifolia M. Bieb. 1819, Fl. Taur.Cauc. 3: 232.

Type: EUROPE, CRIMEA. "In Tauria maxime meridionali circa Nikitam occurens [NK Nikita], 1816, Steven" (lectotype LE!, designated by V. M. Vinogradova 2003: 107).

= Oenanthe media Griseb., 1843, Spic. Fl. Rumel. 1: 352.

$=$ Oenanthe silaifolia M. Bieb. var. media (Griseb.) Beck, 1892, Fl. Nieder-Österr. 2, 1: 641.

Type: EUROPE. "In Macedonia et Serbia: in peninsula Hajion-Oros, Frivaldsky" (lectotype GOET [GOET011377]; designated by Strid 2000: 269; isolectotype BM [BM000751985]); "in pratis pr. Toliewacz, Friedrichsthal 183" (syntype GOET [GOET007372]).

= Oenanthe heldreichii Boiss., 1872, Fl. Orient. (Boissier) 2: 957.

$\equiv$ Phellandrium lobelii Bubani, 1900, Fl. Pyren. (Bubani) 2: 370, nom. illeg. (Art. 52.1).

Type: TURKEY. "In pratis paludosis Pamphyliae ad fluv. Melas. [In pratis paludosis au bord du Melas près du Khan de Monargat]. 02 VII 1845. Heldreich 571" (lectotype G-BOIS!; designated by Pimenov et Jacquemoud, 2020: 48); isolectotype P [P02518787]; "Caria. Pinard" (syntype P [P02518786]).

= Oenanthe brevisecta E. Simon,1903, Rev. Bot. Syst. Géogr. Bot. 1: 96.

Type: TURKEY. "Smyrne, 1854, Balansa 52" (syntype); "Tmolus occid., Yaila de Bozdagh, Balansa" (syntype); "Samsoun, Tchihatschev" (syntype G-BOIS); RUSSIA. "Caucase: Ekaterinodar, gouv. Kuban, Lipsky" (syntype); GREECE. 'Peloponese, Tyrinthe, Orphanides" (syntype); CROATIA. "Istria: Pola, Wawra" (syntype).

= Oenanthe caucasica E. Simon, 1903, Rev. Bot. Syst. Géogr. Bot. 1: 99.
Type: AZERBAIDZHAN. "Littoral de la mer Caspienne; lieux ombrages et humides près de Lenkoran, bouv. de Bakou, 1836, Hohenecker 1842" (syntype); Meyer (syntype).

= Oenanthe biebersteinii E. Simon, 1903, Rev. Bot. Syst. Géogr. Bot. 1: 93, p. min. pte.

= Oenanthe longifoliolata Schischk., 1950, Bot. Mater. Gerb. Bot. Inst. Komarova Akad. Nauk SSSR 13: 164.

Type: AZERBAIDZHAN. "In locis humidis et in paludibis inter Dschairan-Berga et Lenkoran. 15 V 1830. Meyer 1083" (holotype LE!).

= Oenanthe heterococca Korovin, 1948, Not. Syst. Herb. Inst. Bot. \& Zool. Acad. Sci. Uzbekistan 12: 13.

Type: UZBEKISTAN. "Pamiro-alaj occidentalis, in paludosis occidentalem versus urb. Schachrisjabz. 10 VI 1927. Kultiassov \& Granitov 596" (holotype TASH! [TASH002335]), "ibid, ejusd, 621" (isotype TASH [TASH002336]).

Distribution in MA \& $\mathbf{K}$ : Uzbekistan (Qashqadarya prov.).

Distribution in other Asian countries: Russia, Iran, Azerbaidzhan, Georgia, Armenia, Turkey, Lebanon, Syria, Israel.

Distribution area: Asia (Northern, SouthWestern and Mediterranean), Europa, Africa.

Ormopterum turcomanicum (Korovin) Schischk., 1950, in Schischkin (ed.), Fl. URSS 16: 363, 597, tab. 30, fig. 6, 6a.

E Hyalolaena turcomanica Korovin, 1948, Not. Syst. Herb. Inst. Bot. Zool. Acad. Sci. Uzbekistan 12: 21.

三Selinum turcomanicum (Korovin) M. Hiroe, 1979, Umbelliferae World: 1311.

Type: TURKMENISTAN. "Badhyz, in arenosis prope lacus Oerojlan-duz, 16 V 1925, Korovin 355, 356" (holotype TASH! [TASH002356]; designated here, isolectotype TASH! [TASH002357]).

Distribution in MA \& K: Turkmenistan (Akhal prov., Lebap prov., Mary prov.).

Distribution in other Asian countries: Afghanistan.

Distribution area: South-Western Asia.

Osmorhiza aristata (Thunb.) Rydb. 1894, Bot. Surv. Nebraska 3: 37.

$\equiv$ Chaerophyllum aristatum Thunb., 1784, Syst. Veg. ed. 14 (J. A. Murray): 288 et 1784, Fl. Jap. (Thunberg): 119.

$\equiv$ Myrrhis aristata (Thunb.) Spreng., 1813, Neue Schr. Naturf. Ges. Halle 2, 1 (Pl. Umb. Prodr.): 29. 
$\equiv$ Osmorhiza japonica Siebold et Zucc. 1843, Abh. Math.-Phys. Cl. Königl. Akad. Wiss. 4(2): 203, nom. illeg. (Art. 52.1).

$\equiv$ Uraspermum aristatum (Thunb.) Kuntze, 1891, Revis. gen. pl. 1: 270.

$\equiv$ Scandix aristata (Thunb.) Koso-Pol., 1916, Bull. Soc. Imp. Naturalistes Moscou, n. s. 29: 143.

Type: JAPAN. "[presumably from Japan, exact locality unknown], Thunberg" (holotype UPSThunb. [UPS V-007148]).

= Osmorhiza laxa Lindl., 1835, in Ill. Bot. Himal. Mts. (Royle): 233, tab. 52, fig. 1.

$=$ Washingtonia laxa (Lindl.) Koso-Pol., 1920, Izv. Glavn. Bot. Sada R.S.F.S.R. 36(1) (Fl. As. Ross. 15): 52.

= Osmorhiza aristata (Thunb.) Rydb. var. laxa (Lindl.) Shan Renhwa et Constance, 1948, Univ. Calif. Publ. Bot. 23(3): 130.

Type: INDIA. "Punjab, Simore in the Himalayan Mountains [NW India], Royle" (lectotype K! [K000695697], designated by Lowry et Jones 1984: 1150; photo, E [E00265113]; isolectotype K [K000695688]).

= Osmorhiza amurensis F. Schmidt ex Maxim. 1859, Mém. Acad. Imp. Sci. Saint-Petersbourg Divers Savants 9 (Prim. Fl. Amur.): 129.

= Scandix amurensis (F. Schmidt ex Maxim.) Koso-Pol., 1916, Bull. Soc. Imp. Naturalistes Moscou, n. s. 29: 143.

$=$ Washingtonia amurensis (F. Schmidt ex Maxim.) Koso-Pol., 1920, Izv. Glavn. Bot. Sada R.S.F.S.R. 36, 1 (Fl. As. Ross. 15): 50, nom. inval., pro syn.

Type: RUSSIA. "Am untern Amur, in der Nähe der Dondon-Mündung, bei Dshare, an schattigen Laubwaldstellen, zerstreut, 18 VII 1855, Maximowicz" (lectotype LE!; designated by Pimenov, 2017, in Turczaninowia 20, 2: 179; isolectotype K! [K000695689]).

= Washingtonia claytonii (Michx.) Britton subsp. orientalis Koso-Pol., 1920, Izv. Glavn. Bot. Sada R.S.F.S.R. 36, 1 (Fl. As. Ross. 15): 51.

Type: not indicated.

= Washingtonia claytonii (Michx.) Britton subsp. occidentalis Koso-Pol., 1920, Izv. Glavn. Bot. Sada R.S.F.S.R. 36, 1 (Fl. As. Ross. 15): 51.

Type: not indicated.

- Osmorhiza longistylis auct. non DC.: Miq. 1867, Ann. Mus. Bot. Lugduno-Batavi 3: 64.

- Osmorhiza claytonii auct. non Michx.: C. B. Clarke, 1879, in Fl. Brit. India (J. D. Hooker) 2: 690.

Distribution in MA \& K: Kazakhstan (NE: E Kazakhstan prov.).
Distribution in other Asian countries: Russia, China, Japan, Korea, Bhutan, Nepal, India, Pakistan.

Distribution area: Northern, Central and Eastern Asia.

Ostericum palustre (Besser) Besser, 1822, Enum. pl. (Besser): 94.

$\equiv$ Imperatoria palustris Besser, 1809, Prim. fl. Galiciae austriac. 1: 214.

$\equiv$ Angelica palustris (Besser) Hoffm., 1814, Gen.

P1. Umbell.: 162.

三Selinum ostericum E. H. L.Krause, 1904, in Fl. Deutschl. (Sturm), ed. 2 12: 119, fig. 24.

Type: EUROPA, UKRAINE. Leopoli, in pratis uliginosis prope Bogdanowka rara; ad ortum autem sptentrionalem stagni Janowiensis versus Stawki in paludosis frequent. Besser (?) (holotype KWBesser!).

= Ostericum pratense Hoffm. 1816, Gen. P1. Umbell. (ed. 2): 164.

= Angelica pratensis M. Bieb. ex Spreng., 1818, Sp. Umbell.: 60.

Type: UKRAINE. "In pratis depressioribus circa Charkoviam, Marchall von Bieberstein" (holotype MW-Hoffm!).

= Gomphopetalum albiflorum Turcz., 1841, Bull. Soc. Imp. Naturalistes Moscou 14: 540.

= Angelica albiflora (Turcz.) Benth. ex Maxim. 1873, Bull. Acad. Imp. Sci. Saint-Petersbourg 19: 253.

= Ostericum albiflorum (Turcz.) Kitag. 1936, J. Jap. Bot. 12(4-5): 236, in adnot.

Type: RUSSIA. "In viciniis urbis Krasnoyarsk ad rivulos prope pagum Torgaschina locis humidis. [In humidis ad pagum prope Krasnojarsk], Turczaninow" (lectotype LE!; designated here by Pimenov; isolectotypes KW?, K [ K001097156, K001097157], LE!).

Distribution in MA \& K: Kazakhstan (W: Aktobe prov.; N: Kostonai prov., N Kazakhstan prov., NE: E Kazakhstan prov.; Central: Karagandy prov.).

Distribution in other Asian countries: Russia.

Distribution area: Asia (Northern and Central), Europa.

Ostericum tenuifolium (Pall. ex Spreng.) Chu Youchang, 1995, Clavis Pl. Chinae Bor.-Or. (ed. 2): 478, "tenuifolia".

$\equiv$ Athamanta tenuifolia Pall. ex Spreng., 1820, in Syst. Veg. ed. 15 bis (Roemer et Schultes) 6: 495.

$\equiv$ Libanotis tenuifolia (Pall. ex Spreng.) DC., 1830, Prodr. (A. P. de Candolle) 4: 151. 
$\equiv$ Angelica tenuifolia (Pall. ex Spreng.) Pimenov, 1985, Bot. Zhurn. (Leningrad) 70(11): 1494, fig. 1.

Type: RUSSIA. (holotype B-Willd! [BW05743$010]$; designated here; isolectotypes B-Willd! [BW05743-02 0, B-W05743-03 0, B-W05743-04-0]; "In Sibiria versus Ochotsk. Herb. Pallas (Steller [?]) (syntype [?] HAL [HAL0098504]).

= Peucedanum salinum Pall. ex Spreng., 1824, Syst. Veg. (ed. 16) 1: 910.

Type: RUSSIA. "Sibir".

= Oreoselinum humile Besser ex Steud., 1840, Nomencl. Bot., ed. 2, 1: 226.

= Peucedanum humile Turcz. [1838, Bull. Soc. Imp. Naturalistes Noscou 11: 93, nom. inval. (Art. 39.1)], 1844, Bull. Soc. Imp. Naturalistes Moscou 17: 745 .

Type: RUSSIA. "[Baikal \& Transbaikalia] In umbrosis sylvaticis ad rivulorum margines [Ircutia], 1830, 1831, Turczaninow" (syntypes FI! [FI014986], K! [K001097159], LECB [LECB0000079, LECB0000080], M! [M0173148], P! [P00753014P00753017, P02284829, P02818054]).

= Ostericum filisectum Chu Youchang et Liou, 1977, Fl. Pl. Herb. Chin. Bor.-Or. 6: 294, 245, tab. 99.

= Ostericum maximowiczii (F. Schmidt) Kitag. var. filisectum (Chu Youchang et Liou) Yuan Changchi et Shan Renhwa, 1985, Bull. Nanjing Bot. Gard. Mem. Sun Yat Sen, 1984-85: 3.

Type: CHINA. "Nei Mongol: Ergun Banner. 16 VIII 1951. Wang Zhan 1970" (holotype IFP).

Distribution in MA \& K: Kazakhstan (NE: E Kazakhstan prov.).

Distribution in other Asian countries: Ruissia, China.

Distribution area: Arctic, Northern and Eastern Asia.

Pachypleurum alpinum Ledeb., 1829, Fl. Altaic. (Ledebour) 1: 297.

$\equiv$ Ligusticum alpinum (Ledeb.) Kurtz, 1894, Bot. Jahrb. Syst. 19: 464, nom. illeg., non Spreng. (1824).

$\equiv$ Ligusticum mutellinoides (Crantz) Vill. subsp. alpinum (Ledeb.) Thell. 1926, in Hegi, (ed.), Ill. Fl. Mittel.-Eur. 5(2): 1323.

Type: KAZAKHSTAN. "In monte crucis prope Riddersk, regionis subalpinis, Ledebour 248" (syntypes LE!, P! [P03776963, P03776975], PH?); RUSSIA. [Altai] "In alpe Aigulacense. Bunge" (lectotype LE!, designated by V. M. Vinogradova 2005: 963; isolectotypes K [K000695882] P! [P02284825, P03776972, P03776974], PH [PH00018171]).
= Conioselinum gayoides Less. 1834, Linnaea 9: 178.

Type: RUSSIA. "In madidis submuscosis montis Iremel per totam regionem alpinam, altit. 40004700 ped., 1832, Lessing" (holotype LE?).

= Pachypleurum schischkinii Serg. 1956, Sist. Zametki Mater. Gerb. Krylova Tomsk Gosud. Univ. 79-80: 7.

= Pachypleurum alpinum Ledeb. var. schischkinii (Serg.) Malyschev, 1965, Vysokogorn. Fl. Vostochn. Sajana: 188.

Type: KAZAKHSTAN. "Kasachstania orientalis. Systema flum. Kaba, Muzbel, in pratis alpinis, 14 VII 1920, Saposhnikov" (lectotype TK!; designated by A.V. Polozhij \& V.F. Balashova (1989: 26), as "type"; isolectotypes TK).

Distribution in MA \& K: Kazakhstan (NE: E Kazakhstan prov.; SE: Almaty prov.).

Distribution in other Asian countries: Russia, China.

Distribution area: Asia (Arctic, Northern and Central), Europa.

Pachypleurum altaicum Revuschkin, 1990, Sist. Zametki Mater. Gerb. Krylova Tomsk. Gosud. Unin. 88: 1, fig. 1. (contrary to Art 37.7 of the ICBN (2006).

Type: KAZAKHSTAN. "Kazachstania orientalis, jugum Ivanovsky, alpes Proiezdnoi dictae, regio subalpina, ad collem continuum siccum, 29 VII 1985, Revuschkin \& Vydrina" (holotype TK!).

Distribution in MA \& K: Kazakhstan (NE: E Kazakhstan prov.). Endemic.

Distribution area: Northern Asia.

Palimbia defoliata (Ledeb.) Korovin, 1963, in N.V. Pavlov (ed.), Fl. Kazakhst. 6: 382.

$\equiv$ Seseli defoliatum Ledeb., 1829, Fl. Altaic. (Ledebour) 1: 343.

Type: KAZAKHSTAN. "In arenoso-limosis salsis deserti soongoro-kirghisici occidentalis. [Inter fl. Irtysch et montes Tschingistau], VI 1826, Meyer 267" (lectotype LE! [LE01065951], designated by V. M. Vinogradova 2002: 134); isolectotypes C! [C10008579], LE [LE01065952], PH [PH00023775]).

Distribution in MA \& K: Kazakhstan (NE: E Kazakhstan prov.; Central: Karagandy prov.).

Distribution in other Asian countries: China.

Distribution area: Central Asia.

Palimbia rediviva (Pall.) Thell., 1926, in Hegi, (ed.), Ill. Fl. Mittel.-Eur. 5(2): 1364. 
$\equiv$ Peucedanum redivivum Pall., 1779, Nova Acta Petrop. 2: 252, tab. 8.

$\equiv$ Sison salsum L. f. 1782, Suppl. P1.: 181.

$\equiv$ Agasyllis salsa (L. f.) Spreng., 1812, Mag. Neuersten Entdesk. Gesammten Naturk. Ges. Naturf. Freude Berlin 6: 259.

$\equiv$ Siler salsum (L. f.) Spreng., 1818, Sp. Umbell.: 90.

$\equiv$ Ferula salsa (L. f.) Ledeb., 1829, Fl. Altaic. (Ledebour) 1: 344, in nota.

$\equiv$ Palimbia salsa (L. f.) Besser [1822, Enum. pl. (Besser): 55, 93, nomen] ex DC., 1830, Prodr. (A. P. de Candolle) 4: 175.

$\equiv$ Apseudes caspica Raf. 1840, Good Book: 57, nom. inval. (Art. 52.1).

$\equiv$ Peucedanum salsum (L. f.) Steud., 1841, Nomencl. Bot. (Steudel), ed. 2, 2: 311.

$\equiv$ Peucedanum palimbia Baill., 1879, Hist. Pl. (Baillon) 7: 100.

$\equiv$ Foeniculum salsum (L. f.) Calest. 1905, Webbia 1: 205.

$\equiv$ Seseli salsum (L. f.) Koso-Pol., 1916, Bull. Soc. Imp. Naturalistes Moscou, n. s. 29: 183, in nota.

$\equiv$ Ferula rediviva (Pall.) Schischk. 1935, in Krylov, Fl. Sibir. Occ. 8: 1996.

Type: EUROPE, RUSSIA. "In salsis limosis aridissimis circa Wolgam. [In aridis deserti caspiae], Pallas" (lectotype B-Willd! [B-W05860 02 0]; designated here by Pimenov; isolectotypes B-Willd [B-W05860-01 0, B-W05860-03 0], BM, K!, LINN, $\mathrm{M}$ ! [M0173089]).

Distribution in MA \& K: Kazakhstan (W: W Kazakhstan prov., Aktobe prov.; N: Kostonai prov., Akmola prov., Pavlodar prov.; NE: E Kazakhstan prov.; Central: Karagandy prov.).

Distribution in other Asian countries: Russia.

Distribution area: Asia (Northern and Central), Europa.

Palimbia turgaica Lipsky ex Woronow, 1931, Trudy Bot. Sada Akad. Nauk URSS 43, 2 (Flora Rossiae Austro-Orient. 5): 818, descr. ross.

$=$ Palimbia rediviva auct. non Thell.: Schischk., 1951, in Schischkin (ed.), Fl. URSS 17: 54, p. p.

Type: RUSSIA. "Bashkiria, kanton Sterlitamak, Chekleevskoe, pag. Jakchimbetova, 03 VII 1928, Kucherovskaya 281" (lectotype LE!, designated by V. M. Vinogradova 2003: 109); "Pugachev distr., Kuschum Minor ad Irgiz, 25 VII 1927, Terechova" (syntype LE!); “Orenburg prov., Ural'sk - Buzuluk Orenburg, prope pag. Nikolaevka, 06 VI 1927, B. A. Fedtschenko \& Bobrov 193" (syntype LE!); RUSSIA/KAZAKHSTAN. "Orenburg prov., Ilek distr., declivum septentrionale riv. Kindele prope pag. Mustaevo, 18 VIII 1928, Borisova 1543" (syntype LE!).

Distribution in MA \& K: Kazakhstan (W: W Kazakhstan prov.; N: Kostonai prov., N Kazakhstan prov., Akmola prov.; Central: Karagandy prov.).

Distribution in other Asian countries: Russia.

Distribution area: Asia (Northern and Central), Europa.

Paraligusticum discolor (Ledeb.) V. N. Tikhom., 1973, Byull. Moskovsk. Obshch. Isp. Prir. Otd. Biol. 78(1): 107, fig. 4-7.

$\equiv$ Ligusticum discolor Ledeb., 1829, Fl. Altaic. (Ledebour) 1: 321.

$\equiv$ Pleurospermum discolor (Ledeb.) M. Hiroe, 1958, Umbellif. Asia 1: 122.

Type: KAZAKHSTAN. "In pratis v. gr. prope Riddersk, Ledebour 233" (syntype LE! [LE01065782]); “Alexandrowsk, 21 VI 1826, Meyer 266" (lectotype LE! [LE01065781], designated by V. M. Vinogradova 2005: 963; isolectotype P [P03224819]); "Pagum Belaya ad fl. Buchtorma, Ledebour" (syntypes KFTA [KFTA0002588], LE!, $\mathrm{P}$ [P03224819]).

Distribution in MA \& K: Kazakhstan (NE: E Kazakhstan prov.; S: S Kazakhstan prov., Zhambyl prov.; SE: Almaty prov.), Kyrgyzstan (Jalal-Abad prov., Osh prov., Chüh prov.), Tajikistan (Kŭhistani Badakhshan, Sughd prov., Khatlon prov., Dushanbe prov.), Uzbekistan (Toshkent prov.).

Distribution in other Asian countries: China, Mongolia.

Distribution area: Central and South-Western Asia.

Parasilaus asiaticus (Korovin) Pimenov, 1978, Byull. Glavn. Bot. Sada 109: 31.

EScaphospermum asiaticum Korovin, 1951, in Schischkin (ed.), Fl. URSS 17: 358, 292.

Type: TAJIKISTAN. "Tajikistania australis, ditio Duschty-dzum, montes Farkan, trajectus Siun, in rupestribus ad pag. Parvar, alt. 1650-1850 m. 24 VI 1935. Linczevski 541" (holotype LE! [LE01066097]; isotypes LE! [LE01066095, LE01066096]).

= Silaus afghanicus Gilli, 1959, Repert. Spec.

Nov. Regni Veg. 61(3): 197.

$=$ Parasilaus afghanicus (Gilli) Leute, 1972, Oesterr. Bot. Z. 120(3): 291.

Type: AFGHANISTAN. "Nordost-Afghanistan: Pandscherschlucht bei Gulbahar, Nordosthang, Gesteinsspalten unter überhängenden Felsen (Hornblendeschiefer?), 1700 m. 26 V 1950. Gilli 2012" (holotype W [W 1980-0005225]). 
Distribution in MA \& K: Tajikistan (Kŭhistani

Badakhshan, Khatlon prov.).

Distribution in other Asian countries: Afghanistan.

Distribution area: South-Western Asia.

Pastinaca clausii (Ledeb.) Calest. 1905, Webbia 1: 245

$\equiv$ Heracleum clausii Ledeb 1844, Fl. Ross. (Ledebour) 2: 323.

Type: KAZAKHSTAN. "In deserto caspio hinc inde, v. g. circa m. Ischaptschatschi, Claus".

= Pastinaca graveolens M. Bieb. 1808, Fl. Taur.-

Cauc. 1: 237, nom. illeg., non Bernh. (1800).

= Heracleum graveolens (M. Bieb.) Spreng., 1813, Neue Schr. Naturf. Ges. Halle 2(1) (Pl. Umb. Prodr.): 12.

$=$ Malabaila graveolens (M. Bieb.) Hoffm., 1814, Gen. Pl. Umbell.: 126.

= Peucedanum biebersteinii Schmalh., 1895, Fl. Sredn. Yushn. Rossii 1: 411.

= Pastinaca biebersteinii (Schmalh.) Galushko, 1980, Fl. Severn. Kavkaza 2: 272.

Type: EUROPE, CRIMEA. "In arvis et inter segetis imprimis Tauriae [Ex Tauria], 1807, Marchall von Bieberstein" (lectotype LE-Bieb!, designated by Fedoronchuk et Krytzkaja (Vinogradova 2003: 108).

Distribution in MA \& K: Kazakhstan (W: W Kazakhstan prov., Atyrau prov., Aktobe prov.; N: Kostonai prov., Akmola prov., Pavlodar prov.; NE: E Kazakhstan prov.; Central: Karagandy prov.).

Distribution in other Asian countries: Russia.

Distribution area: Asia (Northern and Central), Europa.

Pastinaca sativa L. subsp. sylvestris (Mill.) Rouy et Camus, 1901, in Rouy \& Foucaud (ed.), Fl. France 7: 372, "silvestris".

E Pastinaca sylvestris Mill., 1768, Gard. Dict. (ed. 8): Pastinaca N 1.

$\equiv$ Pastinaca sativa L. var. sylvestris (Mill.) DC., 1830, Prodr. (A. P. de Candolle) 4: 189.

Type: EUROPE, GREAT BRITAIN. "On the side of banks, and on dry land, in many parts of England".

= Pastinaca sativa L. var. arvensis Pers. 1805, Syn. Pl. 1: 322.

Distribution in MA \& K: Kazakhstan (W: Aktobe prov.; N: N Kazakhstan prov.; NE: E Kazakhstan prov.; S: Zhambyl prov.; SE: Almaty prov.), Kyrgyzstan (Talas prov., Chüh prov.), Uzbekistan (Toshkent prov.).
Distribution in other Asian countries: Russia, China, Turkey.

Distribution area: Asia (N, Central and SW), Europa, Africa, N. America (adv.), S. America (adv.), Australia \& Oceania (adv.).

Pastinacopsis glacialis Golosk., 1950, Bot. Mater. Gerb. Bot. Inst. Komarova Akad. Nauk SSSR 12: 198 .

$\equiv$ Pastinaca glacialis (Golosk.) M. Hiroe, 1979, Umbelliferae World: 1759.

Type: KAZAKHSTAN. "Alatau Transiliensis, angustiae fl. M. Alma-Atinka, morenae recentes glacierum Tujuk-su, declivum lapidosum, 3850 m, 06 VIII 1943, Goloskokov" (holotype LE! [LE00052199]; isotypes AA!, LE [LE00052193LE00052198], TASH! [TASH002571]).

Distribution in MA \& K: Kazakhstan (SE: Almaty prov.), Kyrgyzstan (Ysyk-köl prov., Chüh prov.).

Distribution in other Asian countries: China.

Distribution area: Central and South-Western Asia.

Paulita alaica (Pimenov et Kljuykov) Pimenov et Kljuykov, 1986, Bot. Zhurn. (Leningrad) 71(4): 488.

$\equiv$ Neopaulia alaica Pimenov et Kljuykov, 1983, Bot. Zhurn. (Leningrad) 68(11): 1562, fig. 1.

Type: KYRGYZSTAN. "Kyrgyzstan, jugum Alaicum, in gypsaceis rubroarenosis inter oppida Osch et Naukat, trajectus Chokmak, prope pagum Uschbai, 16 VII 1981, Pimenov, Kljuykov, Vasilieva, Baranova, Tomkovich \& Lavrova 365" (holotype LE! [LE01065771]; isotypes MHA!, MW! [MW0593776, MW0593777], TASH! [TASH002230]).

Distribution in MA \& K: Kyrgyzstan (Osh prov.). Endemic.

Distribution area: South-Western Asia.

Paulita alpina (Schischk.) Soják, 1982, Čas. Nár. Muz. Praze, Rada Př́r. 150(1-2): 216.

$\equiv$ Hymenolaena alpina Schischk. 1950, Bot. Mater. Gerb. Bot. Inst. Komarova Akad. Nauk SSSR 13: 158, "aplina".

$\equiv$ Pleurospermum alpinum (Schischk.) M. Hiroe, 1979, Umbelliferae World: 746.

$\equiv$ Paulia alpina (Schischk.) Korovin ex Pimenov et Kljuykov, 1979, Vestnik Mosk. Gosud. Univ. (ser. 16, Biol.) 1: 18, fig. 1-5.

$\equiv$ Neopaulia alpina (Schischk.) Pimenov et Kljuykov, 1983, Bot. Zhurn. (Leningrad) 68(11): 1562. 
Type: TAJIKISTAN. “Asia Media, Tajikistania, in cacumine jugi Vaschsky ad meridiem a trajectu Tundak. 09 VII 1932. Gonczarov, Grigoriev \& Nikitin 604" (holotype LE! [LE00051279]).

Distribution in MA \& K: Tajikistan (Kŭhistani Badakhshan, Khatlon prov., Dushanbe prov.). Endemic.

Distribution area: South-Western Asia.

Paulita ovczinnikovii (Korovin) Soják, 1981, (publ. 1982), Čas. Nár. Muz. Praze, Rada Přír. 150(1-2): 216.

$\equiv$ Paulia ovczinnikovii Korovin, 1973, Izv. Akad. Nauk Tadzh. SSR, Otd. Estest. Nauk 1(50): 14, fig. $1-4$.

$\equiv$ Neopaulia ovczinnikovii (Korovin) Pimenov et Kljuykov, 1983, Bot. Zhurn. (Leningrad) 68(11): 1562 .

Type: TAJIKISTAN. "Karategin, in regione pagum Ramit, 24 VI 1962, Strizhova \& Ivanova 1851" (holotype TAD!; isotype LE! [LE01065772]).

Distribution in MA \& K: Tajikistan (Sughd prov., Khatlon prov., Dushanbe prov.), Uzbekistan (Qashqadarya prov., Surxondarya prov.). Endemic.

Distribution area: South-Western Asia.

Peucedanum morisonii Besser ex Schult., 1820, in Syst. Veg. ed. 15 bis (Roemer \& Schultes) 6: 567.

$=$ Ligusticum longifolium Willd. 1798, Sp. Pl. (ed. 4), 1 (2): 1428, non Peucedanum longifolium Waldst. et Kit.

= Peucedanum ledebourii G. Don, 1834, Gen. Hist. 3: 330.

Type: RUSSIA. "Native in Siberia, everywhere in open situations, particularly on rocks (holotype B-Willd [B-W 05835-01 0, B-W 05835-02 0])

= Peucedanum songoricum Schischk., 1951, in Schischkin (ed.), Fl. URSS 17: 354, 176., nom. illeg., non Peucedanum soongaricum G. Don (1834).

Type: KAZAKHSTAN. "Kazachstania. In jugo Dshungarski Alatau, montes Bajan-Dshurjuk, 03 VII 1909, Lipsky 1798" (holotype LE! [LE00052155]; isotypes LE [LE00052156-LE00052159]).

- Peucedanum officinale auct. non L.: Ledeb., 1829, Fl. Altaic. (Ledebour) 1: 304.

- Peucedanum ruthenicum auct. non M. Bieb.: Krylov, 1935, Fl. Zapadnoi Sibiri 8: 2045.

Distribution in MA \& K: Kazakhstan (N: Kostonai prov., N Kazakhstan prov., Akmola prov., Pavlodar prov.; NE: E Kazakhstan prov.; Central: Karagandy prov.; SE: Almaty prov.).

Distribution in other Asian countries: Russia, China.
Distribution area: Northern and Central Asia.

Peucedanum vaginatum Ledeb., 1829, Fl. Altaic. (Ledebour) 1: 312.

Type: RUSSIA. "In pratensibus ad fl. Irtysch et Koksun, Ledebour" (lectotype LE!; designated by V. M. Vinogradova 2005: 964); isolectotypes HAL [HAL0098524?], LE!, P! [P02272050, P02272051, P02272053], PH? [PH00023074]).

= Peucedanum vaginatum Ledeb. var. pumilum Ledeb., 1829, Fl. Altaic. (Ledebour) 1: 312.

Type: RUSSIA. "In apricis siccissimis deserti Kuraici [Ad Kuraici]. 16-19 VI 1826. Bunge 849" (lectotype LE, designated by V. M. Vinogradova 2005: 964; isolectotypes LE!, P [P02272052]); “Ad fl. Kerlyk, Ledebour" (syntype LE?).

= Peucedanum seseloides Turcz. [1838, Bull. Soc. Imp. Naturalistes Moscou 1: 93, nomen]; ejusd. 1844. Bull. Soc. Imp Naturalistes Moscou 17: 743.

Type:RUSSIA."Ingraminosistransbaicalensibus frequens. 1830. Turczaninow" (lectotype LE!; designated here by Pimenov; isolectotypes K!, M! [M0173140], P [P02272054, P02272055]).

Distribution in MA \& K: Kazakhstan (NE: E Kazakhstan prov.) see Baitulin \& Kotukhov, 2011).

Distribution in other Asian countries: Russia, Mongolia.

Distribution area: Northern Asia.

Phlojodicarpus villosus (Turcz. ex Fisch. et C. A. Mey.) Ledeb., 1844, Fl. Ross. (Ledebour) 2: 331.

$\equiv$ Libanotis villosa Turcz. ex Fisch. et C. A. Mey., 1835, Ind. Sem. Horti Petrop. 1: 31; ejusd., 1836, Linnaea 10, Litt.: 94.

$\equiv$ Stenocoelium villosum (Turcz. ex Fisch. et C. A. Mey.) Koso-Pol., 1916, Bull. Soc. Imp. Naturalistes Moscou, n. s. 29: 132.

$\equiv$ Johrenia villosa (Turcz. ex Fisch. et C. A. Mey.) Kudo, 1923, Rep. Veg. N. Saghal.: 91.

$\equiv$ Phlojodicarpus sibiricus (Fisch. ex Spreng.) Koso-Pol. var. villosus (Turcz. ex Fisch. et C. A. Mey.) Chu Youchang, 1977, Fl. Herb. Chin. Bor.Or. 6: 2897.

$\equiv$ Phlojodicarpus sibiricus (Fisch. ex Spreng.) Koso-Pol. subsp. villosus (Turcz. ex Fisch. et C. A. Mey.) Vorosch. [1982, Opredelitel' Rast. Sovet. Dal'n Vost.: 423, comb. inval.] et 1985, in Florist. Issl. Razn. Raionakh SSSR (A. K. Skvortsov): 184.

Type: RUSSIA. "In montibus trans Baicalem. [In montosis sylvaticis ad Baicalem atque in lapidosis subalpinis prope Schebutui], 1830, Turczaninow" (lectotype LE!; designated by V. M. 
Vinigradova (2005: 963); isolectotypes K! [K0006811171], KFTA [KFTA0001003], KW!, LE!, LECB [LECB0000076], M [M0173080?], P! [P02496408-P02496412]).

= Phlojodicarpus microcarpus Ledeb., 1844, Fl. Ross. (Ledebour) 2: 331.

Type: RUSSIA. "In Sibiriae altaicae montibus Ssojuticus, 1834, Lessing" (holotype LE!).

$=$ Libanotis petrophila Korovin, 1962, Trudy Inst. Bot. Akad. Nauk Kazakhskoi. SSR 13: 255.

Type: KAZAKHSTAN. "Altai, jugum Sarymsakty, fontes fl. Usch-Kungei, 16 VIII 1958, Stepano$v a$ " (holotype AA; designated by V.P. Goloskokov (1963: 29).

Distribution in MA \& K: Kazakhstan (NE: E Kazakhstan prov.).

Distribution in other Asian countries: Russia, Mongolia.

Distribution area: Asia (Arctic, Central and Northern), Europa (E).

Pilopleura goloskokovii (Korovin) Pimenov, 1976, Byull. Glavn. Bot. Sada 101: 50.

三 Platytaenia goloskokovii Korovin, 1962, Trudy Inst. Bot. Akad. Kazakhsk.Kazakhskoi SSR 13: 50.

$\equiv$ Semenovia goloskokovii (Korovin) Bajtenov, 1972, Ill. Opred. Rast. Kazakhstana 2: 108, nom. inval., sine basion. ref.

$\equiv$ Merwiopsis goloskokovii (Korovin) L. K. Safina, 1975, Izv. Akad. Nauk Kazakhskoi SSR, ser. Biol. 5: 35. 1975, nom. inval. (Art. 41.5), without basionym page.

Type: KAZAKHSTAN. "Jugum Dzhungarsky Alatau, pars austro-occidentalis, montes Matai, in system. fl. Matai, 20 VI 1956, Goloskokov" (holotype AA!; isotype LE!).

Distribution in MA \& K: Kazakhstan (SE: Almaty prov.). Endemic.

Distribution area: South-Western Asia.

Pilopleura tordyloides (Korovin) Pimenov, 1976, Byull. Glavn. Bot. Sada 101: 48. 1976.

$\equiv$ Zosima tordyloides Korovin, 1924, Bot. Mater. Gerb. Glavn. Bot. Sada RSFSR 5(6): 82. 1924.

$\equiv$ Platytaenia tordyloides (Korovin) Korovin, 1963, in Pavlov (ed.), Fl. Kazakhst. 6: 423. 1963.

Type: KAZAKHSTAN. "Prov. Syr-Daria, distr. Chimkent, m. Ak-bas-tau, fl. Dengis, pag. Trechsvjatskoe, zona xerophytorum stepposis saxatilibus. 12 VIII 1921. Abolin \& Popov 8169" (lectotype TASH! [TASH002576], designated by Pimenov 1976: 48; isolectotypes LE! [LE00052217], TASH [TASH002575]), "Declivum septemtrionale montium Talas Alatau, Bisch-tasch. 28 VIII 1922. Korovin 1797" (syntypes LE [LE00052216], TASH [TASH002574]; "Prov Syr-darja, distr. Aulie-ata, Mt. Talas Alatau, riv. Kurkursu-Arabik. 04 IX 1921. Abolin \& Popov 8766" (syntypes LE [LE00052218, LE00052219], TASH [TASH002573]).

= Peucedanum dasycarpum Regel et Schmalh., 1878, Trudy Imp. S.-Peterburgsk. Bot. Sada 5(2): 254, nom. illeg. (Art. 53.1), non Torr. et A. Gray (1840).

= Pilopleura koso-poljanskyi Schischk., 1951, in Schischkin (ed.), Fl. URSS 17: 293, 359.

Type: KYRGYZSTAN. "In Turkestaniae valle fluvii Karakol [Karakol, Alatau], VIII 1876, A. Regel" (lectotype LE! [LE00052097], designated by Schischkin 1951: 294; isolectotypes K! [K000695597], LE [LE00052095, LE00052096, LE00052098], P [P00731615]).

= Libanotis talassica Korovin, 1962, Trudy Inst. Bot. Akad. Nauk Kazakhsk. SSR 13: 251.

Type: KAZAKHSTAN. "Tian Schan occidentalis, jugum Talass Alatau, angustiae KschiKaindy, ad ripam dextram, prope frutices Juniperi turkestanicae, in schistosis fixis, 22 VII 1929, Granitov 244" (holotype TASH! [TASH002327]).

Distribution in MA \& K: Kazakhstan (S: S Kazakhstan prov., Zhambyl prov.), Kyrgyzstan (Jalal-Abad prov. Naryn prov., Talas prov., Chüh prov.), Uzbekistan (Toshkent prov.). Endemic.

Distribution area: South-Western Asia.

Pimpinella anisactis Rech. f .Spec. Nov. Regni Veg. 48: 124.

Type: IRAN. "Khorassan, Kopet Dagh inter Quchan et Loftabad, in jugo Allah Akbar, 1800 m, Rechinger 1714" (holotype W [W 1951 00117703]; isotype JE! [JE00002661]).

= Pimpinella litvinovii Schischk. 1950, Bot. Mater. Gerb. Bot. Inst. Komarova Akad. Nauk SSSR 12: 208.

Type: TURKMENISTAN. "Cheirabad, 27 VI 1898, Litvinov 2455" (holotype LE! [LE01065708]; isotype LE [LE01065709].

Distribution in MA \& K: Turkmenistan (Ahal vel.).

Distribution in other Asian countries: Iran.

Distribution area: South-Western Asia.

Pimpinella aurea DC., 1830, Prodr. (A. P. de Candolle) 4: 120.

$\equiv$ Reutera aurea (DC.) Boiss., 1872, Fl. Orient. (Boissier) 2: 863. 
Type: IRAN. "In glareosis Persiae ad Seidkhodzi distr. Khoi prov. Aderbeidzan, 23 VI 1828, Szovits 497" (lectotype G-DC!; designated as "typus" by Engstrand (1987: 315); isolectotype LE!)

= Pimpinella ramosissima DC., 1830, Prodr. 4: 120, nom. illeg. (Art. 52.1), non Fisch. ex Trev. (1819)

$=$ Reutera cervariifolia Boiss., 1844, Ann. Sci. Nat. sér. 3, $2: 134$.

Type: IRAN. "In Persia, Olivier \& Bruguière" (syntype P); "Ispahan, 1837, Aucher-Eloy 3749, 4635" (syntypes FI!, G-BOIS!, P [P00752767]).

= Pimpinella flava C. A. Mey., 1831, Verz. Pfl. Casp. Meer. (C. A. Meyer): 122.

$=$ Reutera flava (C. A. Mey.) Boiss., 1844, Ann. Sci. Nat. sér. 3, 2: 135.

Type: AZERBAIDZHAN. "In locis siccis lapidosis montium Talusch prope pagum Swant (alt. 670 hexap.), Meyer".

Distribution in MA \& K: Turkmenistan (Akhal prov.).

Distribution in other Asian countries: Iran, Azerbaidzhan, Georgia, Armenia, Turkey.

Distribution area: South-Western Asia.

Pimpinella bobrovii (Woronow ex Schischk.) M. Hiroe, 1979, Umbelliferae World: 834.

$\equiv$ Reutera bobrovii Woronow ex Schischk., 1950, in Schischkin (ed.), Fl. URSS 16: 599, 449, tab. 29, fig. 1.

Type: TURKMENISTAN. "Montes Balchany Majores, declivia septentrionalia montibus SekisDagh, in rupibus, 29 VI 1928, Bobrov 135" (lectotype LE! [LE01065712], designated by V. M. Vinogradova 2002: 132).

Distribution in MA \& K: Turkmenistan (Balkan prov.). Endemic.

Distribution area: South-Western Asia.

Pimpinella peregrina L., 1753, Sp. Pl.: 264.

$\equiv$ Apium peregrinum (L.) Crantz, 1767, $\mathrm{Cl}$. umbell. emend.: 101.

$\equiv$ Tragium peregrinum (L.) Spreng., 1813, Neue Schr. Naturf. Ges. Halle 2, 1 (Pl. Umbl. prodr.): 26.

$\equiv$ Carum peregrinum (L.) Baill. ex Koso-Pol., 1916, Bull. Imp. Soc. Naturalistes Moscou 29: 198.

Type: EUROPE, ITALY. "In Italiae pascuis sterilibus, 373.9" (lectotype LINN [LINNHL373-9]; designated by Dawit 1992: 367).

= Caucalis tuberculata Poir., 1811, in Encycl. (J. Lamarck et al.) Suppl. 2: 137.

$=$ Torilis tuberculata (Poir.) Spreng., 1820, in Syst. Veg. ed. 15 bis (Roemer et Schultes) 6: 426.
= Tragium tauricum Ledeb., 1819, Ind. Sem. Horti Dorpat. 6: 286 et Ledeb.ex Link, 1821, Enum. Horti Berol. Alt.: 286.

= Pimpinella taurica (Ledeb.) Steud., 1841, Nomencl. Bot. (Steudel), ed. 2, 2: 336.

$=$ Ledebouria pimpinelloides Link, 1821, Enum. Hort. Berol. Alt. 1: 286, nom. inval.

Type: EUROPA, [Tauria], 2574 Herb. Ledebour" (lectotype LE!: designated here by Pimenov).

= Torilis trilobata Viv. ex DC., 1830, Prodr. (A. P. de Candolle) 4: 121.

= Pimpinella affinis Ledeb., 1844, Fl. Ross. (Ledebour) 2: 257.

Type: "In provinciis transcaucasicis. Eichwald, $359.8 \mathrm{Hb}$. Ledebour" (holotype LE!)

= Pimpinella reuteriana Boiss., 1849, Diagn. Pl. Orient. (ser. 1) 10: 26.

Type: IRAN. "In monte Elbrus [Elburz] Persiae borealis prope Derbent, Kotschy 405" (holotype G?),

= Pimpinella griffithiana Boiss., 1856, Diagn. P1. Orient. (ser. 2) 2: 74.

Type: AFGHANISTAN. "In regno Cabulico, Griffith 897" (lectotype G-BOIS!; designated by Pimenov \& Jacquemoud (2020: 18) ; isolectotype $\mathrm{K}$ ! [K000685584]).

= Pimpinella affinis Ledeb. var. multiradiata Boiss., 1872, Fl. Orient. (Boissier) 2: 868.

= Pimpinella multiradiata (Boiss.) Korovin, 1959, in Vvedensky (ed.), Fl. Uzbekist. 4: 354, nom. illeg. (Art. 53.1), non Santapau (1949).

= Pimpinella korovinii Kamelin, 1971, Novit. Syst. Pl. Vasc. 8: 208.

Type: IRAN. "In dumetis montium Avroman et Schahu Persiae occidentalis Kurdicae [In dumet. Avroman et Schahu, 4-5000'], 06., VI 1867, Haussknecht 462 (lectotype G-BOIS!; designated here by Pimenov et Jacquemoud (2020: 18); isolectotype JE! [JE00003884, JE00003885]).

Note: The isolectotype deposited in G-BOIS with [printed label] "Haussknecht Orient Montes Avromani/Schahu Jul. 1867.

= Pimpinella ambigua K. Koch ex H. Wolff, 1921, Repert. Spec. Nov. Regni Veg. 17: 44.

Type: GEORGIA. "Transcaucasia: KartschChal, Otingo, in valle humido torrentes, a silva densa circumdata, VIII 1895, Rickmer-Rickmers 111 " (syntype B?); TURKEY. "Pontus, in montibus loco non indicato, K. Koch" (syntype B?).

Distribution in MA \& K: Kazakhstan (S: S Kazakhstan prov.), Kyrgyzstan (Batken prov., JalalAbad prov., Osh prov., Chüh prov.), Tajikistan (Kŭhistani Badakhshan, Sughd prov., Khatlon prov., 
Dushanbe prov.), Uzbekistan (Kara-kalpak, Buxoro prov., Navoi prov., Dzhizak prov., Samarqand prov., Qashqadarya prov., Surxondarya prov., Sirdarya prov., Toshkent prov., Andijon prov.), Turkmenistan (Balkan prov., Akhal prov., Lebap prov., Mary prov.).

Distribution in other Asian countries: Russia, Afghanistan, Iran, Azerbaidzhan, Georgia, Armenia, Turkey, Iraq, Saudi Arabia, Lebanon, Syria, Israel, Jordan, Cyprus.

Distribution area: Europa (S), Asia (SW), Africa (N).

Pimpinella puberula (DC.) Boiss., 1844, Ann. Sci. Nat. sér. 3, 1: 129.

$\equiv$ Ptychotis puberula DC., 1830, Prodr. (A. P. de Candolle) 4: 109.

Type: IRAQ. "In Oriente circa Baghdad [de Bagdad a Aleppo; de Baghdad a Kermansha], Olivier \& Bruguière" (lectotype G-DC!; designated here by Pimenov; isolectotypes P! [P00752874, P00752875, P00752882]).

= Pimpinella petraea Nábělek, 1923, Spisy Přir. Fak. Masarikovy Univ. 35: 122, tab. 13, fig. 2, nom. illeg. (Art. 53.1), non Willd. ex Spreng. (1818).

$=$ Pimpinella cretica Poir. var. petraea Zohary, 1972, Fl. Palaestina 2: 427.

= Pimpinella nabelekii M. Hiroe, 1979, Umbelliferae World: 834.

Type: EGYPT, SINAI. "In Arabia Petraea in valle Seil ed Dra inter El-Kerak et Et-Tafile, alt. ca. 500 m, 15 VI 1909, Nábělek 441" (lectotype SAV! [SAV0002324], designated by Pimenov, 2017, in Turczaninowia 20, 2: 192; isolectotypes SAV! [SAV0002322, SAV0002323, SAV0002325]).

$=$ Pimpinella cretica Poir. var. lasiopetala Feinbr., 1931, Repert. Spec. Nov. Regni Veg. 29: 134.

Type: JORDAN. "Mejdel (Gilead). 09 V 1927. Eig, Feinbrun \& Zohary" (syntype); "Umgebung von Amman. 08 V 1927. Eig, Feinbrun \& Zohary" (syntype); “Zwischen Jordan und Es-Salt. 06 V 1927. Eig, Feinbrun \& Zohary" (syntype); "Callirrhoe, 03 IV 1925, Eig, Feinbrun \& Zohary” (syntype).

Distribution in MA \& K: Kazakhstan (Central: Kzyl-Orda prov.; S: S Kazakhstan prov., Zhambyl prov.), Kyrgyzstan (Batken prov., Jalal-Abad prov., Osh prov.), Tajikistan (Sughd prov., Khatlon prov., Dushanbe prov.), Uzbekistan (Buxoro prov., Dzhizak prov., Samarqand prov., Qashqadarya prov., Surxondarya prov., Sirdarya prov., Toshkent prov., Namangan prov., Fergona prov., Andijon prov.), Turkmenistan (Balkan prov., Akhal prov., Mary prov.).

Distribution in other Asian countries: China, Pakistan, Afghanistan, Iran, Azerbaidzhan, Turkey, Iraq, Kuwait, Qatar, Saudi Arabia, UAE, Yemen, Syria, Israel, Jordan.

Distribution area: Central, South-Western and W Tropical Asia.

Pimpinella saxifraga L., 1753, Sp. P1.: 263.

三Apium tragoselinum Crantz, 1767, Cl. Umbell. emend.: 100, nom. illeg. (Art. 52.1).

$\equiv$ Tragoselinum minus Lam., 1779, Fl. franç. Lamarck) 3: 447, nom. illeg. (Art. 52.1).

三Tragoselinum saxifragum (L.) Moench, 1794, Methodus (Moench): 99.

$\equiv$ Pimpinella variifolia Salisb., 1796., 1896, Prodr. stirp. Chap. Allerton: 168, nom. illeg. (Art. 52.1).

$\equiv$ Pimpinella minor Erhr. ex Spreng., 1820, in Syst. Veg. ed. 15 bis (Roemer \& Schultes), 6: 386, nom. illeg. (Art. 52.1).

$\equiv$ Carum saxifraga (L.) Benth. 1867, in Gen. P1. (Bentham \& Hooker f.) 1(3): 892.

$\equiv$ Apium pimpinella Caruel, 1886, in F1. Ital. (Parlatore) 8: 452, nom. illeg. (Art. 52.1).

$\equiv$ Selinum pimpinella E. H. L. Krause, 1904, in Fl. Deutschl. (Sturm) ed. 2 12: 53, fig. 8.

$\equiv$ Apium saxifragum (L.) Calest. 1905, Webbia 1: 178 .

Type: EUROPE. "In Europae pascuis siccis, Saxifraga 1, 373.1" (lectotype LINN [LINNHL373-1], designated by Reduron et Jarvis in Jarvis et al., 1993: 76).

= Pimpinella calvertii Boiss., 1856, Diagn. P1. Orient. (ser. 2) 2: 73.

$=$ Pimpinella saxifraga L. var. dissectifolia Boiss., 1872, Fl. Orient. (Boissier) 2: 873.

Type: TURKEY. "Circa Erzeroum, Calvert 748" (lectotype G-BOIS; designated by Pimenov et Jacquemoud (2020: 21); isolectotypes G-BOIS!, JE! [JE00002662]).

- Pimpinella major auct. non Huds.: Tamamsch., 1967, in Fl. Kavkaza (Grossheim) ed. 2 7: 85, map 93.

- Pimpinella dissecta auct. non Retz.: Vodop., 1979, in Fl. Tsentral'noi Sibiri 2: 678.

Distribution in MA \& K: Kazakhstan (W: W Kazakhstan prov., Aktobe prov.; N: Kostonai prov.).

Distribution in other Asian countries: Russia, Iran, Azerbaidzhan, Georgia, Armenia, Turkey, Iraq. 
Distribution area: Asia (Northern, Central and South-Western), Europa, N. America.

Pimpinella tragium Vill., 1779, Prosp. Hist. pl. Dauphiné: 24. 1779.

$\equiv$ Tragium columnae Spreng., 1813, Neue Schr. Naturf. Ges. Halle 2(1) (Pl. Umb. Prodr.): 26.

$\equiv$ Tragium petraeum Hoffm., 1814, Gen. P1. Umbell.: 91 .

$\equiv$ Apium tragium (Vill.) Caruel, 1889, in Fl. Ital. (Parlatore) 8: 460.

Type: EUROPE, S FRANCE. "Dauphiné, Villars 442" (syntypes BM [BM000049613 BM000049616], designated by O. V. Yurtzeva 1995: 64).

= Pimpinella pseudotragium DC., 1830, Prodr. (A. P. de Candolle) 4: 122.

= Pimpinella tragium Vill. var. pseudotragium (DC.) Boiss., 1872, Fl. Orient. (Boissier) 2: 872.

= Pimpinella tragium Vill. subsp. pseudotragium (DC.) V. A. Matthews, 1972, in Davis (ed.), Fl. Turkey 4: 361.

Type: IRAN. "In apricis siccis lapidosis circa Seidchadzi, distr. Khoi, prov. Aderbeidschan, $18 \mathrm{VI}$ 1828, Szovits 467" (lectotype G-DC!; designated here by Pimenov; isolectotype LE! [LE00015722]).

= Tragium depressum Sieber ex Spreng., 1820, Syst. Veg, ed. 15 bis (Roemer \& Schultes) 6: 392.

= Pimpinella depressa (Sieber ex Spreng.) DC., 1830, Prodr. (A. P. de Candolle) 4: 120.

$=$ Pimpinella tragium Vill. var. depressa (Sieber ex Spreng.) Boiss., 1872, Fl. Orient. (Boissier) 2: 831.

= Pimpinella tragium Vill. subsp. depressa (Sieber ex Spreng.) Tutin, 1968, Feddes Repert. 79(1-2): 62.

Type: EUROPE, GREECE. "Insula Creta, Mt. Sphar. 1817, Sieber 144" (syntypes FR [FR0044739], G, JE [JE00015430]).

= Pimpinella tragium Vill. var. laciniata DC., 1830, Prodr. (A. P. de Candolle) 4: 171

= Pimpinella triradiata Boiss., 1849, Diagn. Pl. Orient. (ser. 1) 10: 27.

$=$ Pimpinella tragium Vill. var. depauperata Boiss., 1872, Fl. Orient. (Boissier) 2: 872.

Type: LEBANON. "Syria, inter Ainete et Deir el Achmar. V-VI 1846. Boissier" (lectotype G-BOIS; designated by Pimenov \& Jacquemoud (2020: 20); isolectotype P [P02519764]).

= Pimpinella polyclada Boiss. et Heldr. 1856, Diagn. Pl. Orient. (ser. 2) 2: 75.

$=$ Pimpinella tragium Vill. var. polyclada (Boiss. et Heldr.) Boiss., 1872, Fl. Orient. (Boissier) 2: 872.
$=$ Pimpinella tragium Vill. subsp. polyclada (Boiss. et Heldr.) Tutin, 1968, Feddes Repert. 79(12): 62 .

Type: EUROPE, GREECE. "In regione sylvaticâ Olympi Thessaliae, 31 VII 1851, Heldreich 2457" (lectotype G-BOIS!, designated by O. V. Yurtzeva 1995: 65; isolectotypes BP!, FI!, GOET [GOET011389, GOET011390], LE!, WAG [WAG0004956]); "In monte Parnasso. In reg. abietina Hagios Nicolaos (Kalkaria), 15 VIII 1852, Heldreich 2691, 2692, 2693" (syntypes G-BOIS!, $\mathrm{W}$ [W0062486]).

= Pimpinella zagrosica Boiss. et Hausskn., 1872, in Fl. Orient. (Boissier) 2: 872.

Type: IRAN. "In montibus Avroman et Schahu Persiae occid. alt. 5000'-7000', VII 1867. Haussknecht [464?]" (lectotype G-BOIS!; designated by Pimenov \& Jacquemoud (2020: 21); isolectotypes JE! [JE00005821, JE00005822], K! [K000685571]).

= Pimpinella anthriscoides Boiss. var. dissecta Kuntze, 1887, Trudy Imp. S.-Peterburgsk. Bot. Sada 10: 192.

Type: TURKMENISTAN. "Turkmen-Steppe. 06.1886. Kuntze" (syntypes NY [NY00406190, NY00406191]).

= Peucedanum pimpinelloides Boiss. et Hausskn. 1888, in, Fl. Orient. (Boissier) Suppl.: 268.

Type: IRAN. "In graniticis montis Elvend, Haussknecht 484c" (lectotype G-BOIS; designated here by Pimenov et Jacquemoud (2020: 66); isolectotype JE!).

= Pimpinella tragium Vill. var. cyprica $\mathrm{H}$. Wolff, 1927, in Pflanzenr. (Engler) Umbellif.-Apioid.Ammin.) 90(IV, 228): 251.

Type: CYPRUS. "Cypern, an Felsen oberhalb Bellapais [Ad rupes pr. Bellapais], 1880, Sintenis \& Rigo 592" (syntypes K [K000685568, K000685569], LD [LD1215911]).

= Pimpinella tragium Vill. var. afghana H. Wolff, 1927, in Pflanzenr. (Engler) Umbellif.-Apioid.Ammin. 90(IV, 228): 253.

Type: AFGHANISTAN. "Kurum-Tal, Aitchison, 738, 748, 930".

= Pimpinella idae Takht. 1940, Zametki Sist. Geogr. Rast. 9: 24.

Type: GEORGIA. "Abkhasia, in faucibus fl. Bzyb, in fissuris rupium calcareorum, 07 VIII 1939, Takhtajan 668" (holotype TBI! [TBI1024322]).

= Pimpinella grossheimii Schischk. 1950, Bot. Mater. Gerb. Bot. Inst. Komarova Akad. Nauk SSSR 12: 204. 
Type: AZERBAIDZHAN. "Talysh, steppa altimontana inter pagos Aschurat [Amurat] et Dygya, alt. ca. 1500 m, 13 VI 1894, Lomakin" (lectotype LE!; designated here by Pimenov; isolectotype TBI! [TBI1024319]).

= Pimpinella daghestanica Schischk. 1950, Bot. Mater. Gerb. Bot. Inst. Komarova Akad. Nauk SSSR 12: 203.

Type: RUSSIA. "Prov. Daghestan, in declivibus montanis inter opp. Machacz-kala (ol. Petrovsk) and Buinaksk (ol. Temir-chan-Schura). 29 VI 1940. Gordjagin" (holotype LE!).

= Pimpinella lithophila Schischk. 1950, Bot. Mater. Gerb. Bot. Inst. Komarova Akad. Nauk SSSR 12: 206.

$=$ Pimpinella tragium Vill. subsp. lithophila (Schischk.) Tutin, 1968, Feddes Repert. 79(1-2): 62.

Type: EUROPE, "Tauria, Staryi Krym, in declivitatibus montis Agar-mysch. 17 VIII 1927. Tzyrina" (holotype LE!).

= Pimpinella turcomanica Schischk. 1950, Bot. Mater. Gerb. Bot. Inst. Komarova Akad. Nauk SSSR 12: 207.

Type: TURKMENISTAN. "Kopet-Dagh, vicinitas urb. Karakala, angustiae Ioldere, declivum australe montis Chosar-Dagh, 1200-1500 m s. m. 23 VI 1931. Linczevski 334" (holotype LE! [LE01065710]; isotype LE! [LE01065711]).

Distribution in MA \& K: Kazakhstan (W: W Kazakhstan prov., Aktobe prov.), Turkmenistan (Balkan prov., Akhal prov.).

Distribution in other Asian countries: Russia, Iran, Azerbaoidzhan, Georgia, Armenia Turkey, Iraq, Lebanon, Syria.

Distribution area: Asia (Northern, SouthWestern and Mediterranean), Europa, Africa (N).

Pleurospermum uralense Hoffm., 1814, Gen. P1. Umbell.: IX, tab. 1B, 2.

$\equiv$ Pleurospermum austriacum (L.) Hoffm. subsp. uralense (Hoffm.) Horn, 1946, Svensk Bot. Tidskr. 40: 182 .

Type: RUSSIA. "Hortus Gorenk. et botanicum Mosquens introducto F. G. Helm".

= Pleurospermum camtschaticum Hoffm., 1814, Gen. Pl. Umbell.: X.

= Ligusticum kamtschaticum (Hoffm.) Steud., 1821, Nomencl. Bot. (Steudel): 478.

Type: RUSSIA. "Kamtschatka, Langsdorff" (holotype LE!).

$=$ Laserpitium athamantae Spreng., 1820, in Syst. Veg. ed. 15 bis (Roemer \& Schultes) 6: 624.
Type: RUSSIA. "In Sibiria".

= Pleurospermum archangelica Ledeb., 1829, Fl. Altaic. (Ledebour) 1: 369.

Type: KAZAKHSTAN. "In Sibiria altaica ad rivulorum margines circa Riddersk. Ledebour".

- Pleurospermum austriacum auct. non Hoffm.: Ledeb., 1829, Fl. Altaic. (Ledebour) 1: 368.

Distribution in MA \& $\mathbf{K}$ : Kazakhstan $(\mathrm{N}$ : Kostonai prov. Akmola prov.; NE: E Kazakhstan prov.).

Distribution in other Asian countries: Russia, China, Japan, Korea, Mongolia

Distribution area: Northern, Central and Eastern Asia.

Prangos ammophila (Bunge) Pimenov et V. N. Tikhom., 1981, in S. K. Cherepanov, Sosud. Rast. SSSR: 27.

ECryptodiscus ammophilus Bunge, 1852, Beitr. Fl. Russl. (Lehm Rel. Bot.): 139.

$\equiv$ Cachrys ammophila (Bunge) Koso-Pol., 1916, Bull. Soc. Imp. Naturalistes Moscou, n. s. 29: 163.

$\equiv$ Neocryptodiscus ammophilus (Bunge) Hedge et Lamond, 1987, in Flora Iranica (Rechinger) 162: 208.

Type: UZBEKISTAN. "Im Flugsande des Batkak-kum, 21 IV 1842, Lehmann 569" (holotype LE! [LE00050137]; isotypes LE [LE00050135, LE00050136]).

$=$ Cachrys korolkowii Regel et Schmalh., 1878, Trudy Imp. S.-Peterburgsk. Bot. Sada 5(2): 602.

Type: UZBEKISTAN. "Inter Chala-ati et Adam Kir-Ullan in solo arenoso [Chiva]. 30 IV 1873. Korolkov" (holotype LE! [LE00050139]; isotype LE [LE00050138]).

Distribution in MA \& K: Uzbekistan (Karakalpak, Khorezm prov., Buxoro prov., Navoi prov.), Turkmenistan (Balkan prov., Daşoguz prov., Akhal prov.). vel.). Endemic.

\section{Distribution area: Central Asia.}

Prangos bucharica B. Fedtsch., 1899, Bull. Herb. Boissier 7: 179.

$\equiv$ Cachrys bucharica (B. Fedtsch.) Herrnst. et Heyn, 1975, Notes Roy. Bot. Gard. Edinburgh 33(3): 443.

Type: TAJIKISTAN. "Buchara, in montibus Chirmat, 1897, Geyer" (holotype LE!).

= Prangos afghanica Podlech, 1970, Mitt. Bot. Staatsamml. München 8: 175.

Type: AFGHANISTAN. "Nordost-Afghanistan, Prov. Takhar: ca. $5 \mathrm{~km}$ südlich von Iskamish, $1300 \mathrm{~m}$, Lössboden, 14 V 1965, Podlech 10640" (lectotype 
$\mathrm{M}$ ! [M0172830]; designated here; isolectotypes M [M0172831], MSB [MSB003000, MSB003001]).

Distribution in MA \& K: Tajikistan (Khatlon prov., Dushanbe prov.), Uzbekistan (Qashqadarya prov., Surxondarya prov.), Turkmenistan (Labap prov.).

Distribution in other Asian countries: Afghanistan.

Distribution area: South-Western Asia.

Prangos cachroides (Schrenk) Pimenov et V. N. Tikhom., 1981, in S. K. Cherepanov, Sosud. Rast. SSSR: 28.

$\equiv$ Cryptodiscus cachroides Schrenk, 1841, Enum. Pl. Nov. 1: 65.

$\equiv$ Cachrys cryptodiscus Koso-Pol., 1916, Bull. Soc. Imp. Naturalistes Moscou, n. s. 29: 163, nom. illeg. (Art. 52.1).

$\equiv$ Neocryptodiscus cachroides (Schrenk) V. M. Vinogr., 1994, in Grubov (ed.), Rast. Tsentr Azii 10: 59.

Type: KAZAKHSTAN. "Semina matura ad lacum Alakul lecta. 13 VII 1840. [In collibus sabulosis Songoriae, inter fontem Sassykpastau et montes Arganaty, 1841], Schrenk" (holotype LE).

$=$ Cryptodiscus rutifolius Bunge, 1852, Beitr. Fl. Russl. (Lehm. Rel. Bot.): 139.

= Cachrys rutifolia (Bunge) Koso-Pol., 1916, Bull. Soc. Imp. Naturalistes Moscou, s. n. 29: 163.

Type: KAZAKHSTAN. "Auf der Lehmsteppe zwischen dem Kuwan- und Syr-Darja. 09 V 1842, Lehmann" (syntype LE [LE00051338]; isolectotype LE [LE00051337]); "Häufig im Sande bei Malibasch am Syr-Darja, 13 V 1842, Lehmann 566" (lectotype LE! [LE00051339]; designated by V. M. Vinogradova 1999: 84); "Zwischen Malibasch und dem See Aigirak und auf Lehmflächen und Sandhügeln am Syr-Darja und Aigirak-See. 15 V 1842. Lehmann" (syntypes).

Note: It is very possible that three sheets kept in $\mathrm{P}$ under the barcodes P00834635, P00834636, and P00834685 also belong to type materials of Cryptodiscus rutifolius.

= Cachrys didyma Regel, 1878, Trudy Imp. S.Peterburgsk. Bot. Sada 5(2): 601.

= Cryptodiscus didymus (Regel) Korovin, 1924, Byull. Sredne-Asiatsk. Gosud. Univ. 7, Suppl. (Sched. Herb. Fl. As. Med. 1-2): 23.

=Prangos didyma (Regel) Pimenov et V. N. Tikhom., 1981, in S. K. Cherepanov, Sosud. Rast. SSSR: 28.

$=$ Neocryptodiscus didymus (Regel) Hedge et Lamond, 1987, in Flora Iranica (Rechinger) 162: 208.
Type: TAJIKISTAN. "In montibus karatavicis Mogol-tau. 20 IV 1868. Sewerzov" (lectotype LE! [LE00051350], designated by A. M. Geldykhanov 1992: 104).

$=$ Cachrys didyma Regel var. glabra Regel, 1878, Trudy Imp. S.-Peterburgsk. Bot. Sada 5(2): 601.

Type: KAZAKHSTAN. "Desertum Aralense. Regio fluv. Ssyr-Darja, inter Murtur et Karabogut. 14 V 1858. Borszczow 729" (lectotype LE! [LE00051352]; designated here by Pimenov; isolectotype LE! [LE00051351]).

= Cryptodiscus arenarius Schischk., 1950, in Schischkin (ed.), Fl. URSS 16: 594, 263.

$=$ Cachrys arenaria (Schischk.) M. Hiroe, 1979, Umbelliferae World: 420.

$=$ Prangos arenaria (Schischk.) Pimenov et V. N. Tikhom., 1981, in S. K. Cherepanov, Sosud. Rast. SSSR: 28.

Type: KAZAKHSTAN. "Asia Media, arenae Mujunkum pars austro-orientalis, in Artemisietocollium arenosorum. 14 VI 1935. Gael \& Kolikov" (holotype LE! [LE00051334]; isotype LE [LE00051333]).

Distribution in MA \& K: Kazakhstan (W: Aktobe prov.; NE: E Kazakhstan prov.; Central: Karagandy prov., Kzyl-Orda prov.; S: S Kazakhstan prov., Zhambyl prov.; SE: Almaty prov.), Kyrgyzstan (Jalal-Abad prov., Chüh prov.), Tadjikistan (Soghd prov.), Uzbekistan (Kara-kalpak, Buxoro prov., Navoi prov., Dzhizak prov., Sirdarya prov., Toshkent prov.), Turkmenistan (Lebap prov., Mary prov.).

Distribution in other Asian countries: China, Afghanistan

Distribution area: Central and South-Western Asia.

Prangos dzhungarica Pimenov, 1983, in Vvedensky (ed.), Opred. Rast. Sred. Azii 7: 371, 203.

Type: KAZAKHSTAN. "Prov. Taldy-Kurgan, Montes Dzhungar Alatau, declivum septentrionale, valle fl. Rgaity, declive lapidosum, 29 VI 1974, Pimenov 476" (holotype MW! [MW0593780]; isotypes LE! [LE00051384, LE00051385, LE00051386]).

$=$ Prangos multicostata Kljuykov et Lyskov, 2016, Phytotaxa 277(1): 70.

Type: KAZAKHSTAN. "East Kazakhstan province: Urzhar destrict, vicinity of Kysyl-Bulak village, Tarbagatai Range (S side, valley of Kokterek river, gravelly slope, alt $=830-910 \mathrm{~m}, 47^{\circ} 00^{\prime} \mathrm{N}$, $82^{\circ} 17^{\prime}$ E. 01 VI 2010. Maslova \& Khrustaleva" (holotype MW! [MW095558, MW0595559]).

Note: Type material of the last species is represented by evidently terate specimen with synflorescence the most probably infected by insects 
(probably additional mericarp ribs, not typical for the genus Prangos, have the same origin). Difference in length of terminal leaves lobes may be connected with ecological conditions and vegetation in different years. Position P. multicostata in subclade of molecular tree together with P. didyma and P. cachroides (two names of the same species) is in clear incongruence with basic carpological characters of three species. So, the separation of $P$. multicostata seems to be dubious and at least praemature. The isotypes of $P$. dzhungarica in LE are more similar to P. multicostata than those in MW

Distribution in MA \& K: Kazakhstan (NE: E Kazakhstan prov.; SE: Almaty prov.). Endemic.

Distribution area: Central and South-Western Asia.

Prangos equisetoides Kuzmina, 1962, Bot. Zhurn. (Leningrad) 47(2): 252, in adnota.

Type: KAZAKHSTAN. "Tian-Schan occidentalis, jugum Kara-tau in faucibus Kok-saraj. In declivibus schistosis, alt. $500 \mathrm{~m} \mathrm{~s} .1$. m., 04 VI 1958, Nevstroeva, Markova \& Medvedeva" (syntypes LE! [LE00051387-LE00051395]).

Distribution in MA \& K: Kazakhstan (Central: Kzyl-Orda prov.; S: S Kazakhstan prov.). Endemic.

Distribution area: Central and South-Western Asia.

Prangos fedtschenkoi (Regel et Schmalh.) Korovin, 1948, Not. Syst. Herb. Inst. Bot. Zool. Acad. Sci. Uzbekistan 12: 24.

$\equiv$ Hippomarathrum fedtschenkoi Regel et Schmalh., 1878, Trudy Imp. S.-Peterburgsk. Bot. Sada 5(2): 603.

Type: Tajikistan. "In Turkestania, prope Chodschent. 04 VI 1871. O. A. Fedtschenko" (lectotype LE! [LE00051327], designated by V. M. Vinogradova (2001: 52); isolectotypes G! [G00367238], LE [LE00051326]; "Steppe prope Maibulak. Krause" (syntype LE [LE00051325], "Kokand, inter Jordan et Karakasuk. 09 VII 1871. O. A. Fedtschenko" (syntypes LE [LE00051323, LE00051324]).

= Prangos pachypoda Korovin, 1924, Bot. Mater. Gerb. Glavn. Bot. Sada R.S.F.S.R. 5(6): 73.

Type: Tajikistan. "Tian-Schan occidentalis, in mont. Mogol-tau, prope opp. Chodschent, 19 VI 1923, Popov \& Vvedensky 172, 173, 175, 176, 177" (lectotype LE! [LE00051406], designated by V. M. Vinogradova (2001: 57); isolectotypes LE [LE00051407], MHA!); UZBEKISTAN. "Prov. Fergana, prope Dragomirovo, 29 VI 1920. Popov
756 " (syntype TASH [TASH002262]; TAJIKISTAN. "Chodschent, 24 IV 1916, Betger" (syntype TASH! [TASH002261, TASH002263]).); UZBEKISTAN. "Dschisak, 1921, Vvedensky".

Distribution in MA \& K: Kyrgyzstan (Batken prov., Osh prov.), Tajikistan (Sughd prov., Dushanbe prov.), Uzbekistan (Dzhizak prov., Qashqadarya prov., Samarqand prov., Surxondarya prov.). Endemic.

Distribution area: South-Western Asia.

Prangos herderi (Regel) Herrnst. et Heyn, 1977, Boissiera 26: 63, fig. 17.

$\equiv$ Cachrys herderi Regel, 1877, Trudy Imp. S.Peterburgsk. Bot. Sada 5(2): 601

Type: KAZAKHSTAN. "In Turkestaniae orientalis montibus alatavicis cis-iliensibus Kara Tschek, Semenov" (holotype LE! [LE00051353]).

Distribution in MA \& K: Kazakhstan (NE: E Kazakhstan prov.; SE: Almaty prov.).

Distribution in other Asian countries: China.

Distribution area: Central and South-Western Asia.

Prangos lachnantha (Korovin) Pimenov et Kljuykov, 1986, Byull. Moskovsk. Obshch. Isp. Prir. Otd. Biol. 91(6): 110.

ESchrenkia lachnantha Korovin, 1962, Trudy Inst. Bot. Akad. Nauk Kazakh. SSR 13: 244.

Type: KAZAKHSTAN. "Kazakh SSR, desertum transkaratavicum, planities argillosa, artemisiosa prope oppidum Tschulak-kurgan. $06 \mathrm{~V} 1930$. N. V. Pavlov 188" (holotype AA!; isotype MW! [MW0593749]).

Distribution in MA \& K: Kazakhstan (S: Zhambyl prov.). Endemic.

Distribution area: Central Asia.

Prangos latiloba Korovin, 1924, Bot. Mater. Gerb. Glavn. Bot. Sada R.S.F.S.R. 5(6): 74.

$\equiv$ Cachrys latiloba (Korovin) Herrnst. et Heyn, 1975, Notes Roy. Bot. Gard. Edinburgh 33(3): 443.

Type: TURKMENISTAN. "Montes KopetDagh, in regione alpina et inferiore, prope Kaakha, 01 V 1914, Korovin E 540" (holotype TASH! [TASH003702]).]; designated here by Pimenov et Kljuykov).

Distribution in MA \& K: Turkmenistan (Balkan prov., Akhal prov.).

Distribution in other Asian countries: Afghanistan, Iran.

Distribution area: South-Western Asia. 
Prangos ledebourii Herrnst. et Heyn, 1977, Boissiera 26: 68, fig. 20.

$\equiv$ Cachrys macrocarpa Ledeb., 1829, Fl. Altaic. (Ledebour) 1: 364.

Type: KAZAKHSTAN. "In collibus apricis et rupestribus deserti songoro-kirghisici ex adverso fortalitii Ustkamenogorsk et alibi, Ledebour" (syntypes C [C10008532], HAL [HAL0098493], LE, PH [PH00010104]); "Inter fortalitium Buchtorminsk et lacum, qui Noor-Saisan vocatur [Altai. In campestribus et collibus apricis petrosis a fortalitio Buchtarminsk orientem versus usque ad montes Dolen-Kara et Arkaul], 1826, Meyer 228" (lectotype LE! [LE00051354], designated by V. M. Vinogradova 1999: 83); In collibus deserti Korgisorum. Herb. Ledebour. 09 V 1826 [?], coll. unclear 160" (syntypes LE [LE00051355LE00051363]).

Distribution in MA \& K: Kazakhstan $(\mathrm{N}$ : Akmola prov.; NE: E Kazakhstan prov.; Central: Karagandy prov.; SE: Almaty prov.).

Distribution in other Asian countries: China, Mongolia.

Distribution area: Central Asia.

Prangos lipskyi Korovin, 1927, Byull. SredneAsiatsk. Gosud. Univ. 15, Suppl.: 49, in clavi. [Schedae Herb. Fl. Asia Med. N 319b].

Type: KYRGYZSTAN. "[M. Ferganenses] Fergana, valle fl. Arslanbob, ad ripam sinistram, 12 VII 1926, Korovin 331, 332" (lectotype TASH! [TASH002249, TASH002250]; designated here).

= Prangos isphairamica B. Fedtsch., 1950, in Schischkin (ed.), Fl. URSS 16: 594, 267.

Type: KYRGYZSTAN. "Asia Media, Jugum Alaicum in systemate flum. Isphairam, in rupestribus as ripam flum. Iniczke. 10 VIII 1915. Drobov 474" (lectotype LE! [LE00051402]; designated by V. M. Vinogradova (2001: 56); isolectotype TASH! [TASH002245, TASH002246]).

Distribution in MA \& K: Kyrgyzstan (Batken prov., Jalal-Abad prov., Osh prov.), Uzbekistan (Namangan prov.). Endemic.

Distribution area: South-Western Asia.

Note: The first brief description of Prangos lipskyi was placed on the label of Schedae in the determination key to the Middle Asian representatives of the genus. The type specimen was not designated; only it is noted that the species is distributed in "m. Ferganenses". B. A. Fedtschenko (1950: 267) described the species only in Russian and considered it as endemic to $\mathrm{W}$ Tianshan, the type of which is in Tashkent. Type specimen was not designated also in Korovin (1959), Kuzmina (1962: 252) and Pimenov \& Tikhomirov (1983: 206); it was selected only by Pimenov \& Kljuykov (2002a) as "type".

Prangos odontalgica (Pall.) Herrnst. et Heyn, 1977, Boissiera 26: 66, fig. 18.

$\equiv$ Cachrys odontalgica Pall., 1776, Reise Russ. Reiches 3: 720.

Type: EUROPE, RUSSIA. "In desertis limosis aridissimis inter Volgam et Iaicam, Pallas (lectotype LINN [LINN-HL349-4] ex Herb. Pallas]; designated here, isolectotypes BM, C [C10008372, C10008373], HAL [HAL0098491], LE! [LE00051368], LIV!).

$=$ Cachrys callosperma Pall., 1776, Reise Russ. Reich. 3(2): 663.

- Cachrys libanotis auct. non L.: Georgi, 1800, Beschr. Russ. Reich 3(4): 845.

Distribution in MA \& K: Kazakhstan (W: W Kazakhstan prov., Atyrau prov., Aktobe prov.; Central: Karagandy prov., Kzyl-Orda prov.).

Distribution in other Asian countries: Russia.

Distribution area: Asia (Northern and Central), Europa.

Prangos ornata Kuzmina, 1962, Bot. Zhurn. (Leningrad) 47(2): 252, in adnota.

Type: KAZAKHSTAN. "Tian-Schan occidentalis, prope pagum Iskander. 15 VII 1897. O. A. Fedtschenko" (holotype LE! [LE00051404, LE00051405]).

= Prangos quasiperforata Kuzmina, Bot. Zhurn. (Leningrad) 47(2): 252. 1962, in adnota.

Type: KYRGYZSTAN. "[Turkestan] Alabuga (Naryn), 7000-8000', 04 VI 1880, A. Regel" (holotype LE! [LE00051411]; isotypes LE! [LE00051408-LE00051410]).

- Prangos uloptera auct. non DC.: Korovin, 1927, Byull. Sredne-Asiatsk. Gosud. Univ. Suppl. 15: 49.

Distribution in MA \& K: Kazakhstan (S: S Kazakhstan prov., Zhambyl prov.), Kyrgyzstan (Jalal-Abad prov., Talas prov., Chüh prov.), Tajikistan (Sughd prov.), Uzbekistan (Navoi prov., Namangan prov., Samarqand prov., Toshkent prov.). Endemic.

Distribution area: South-Western Asia.

Prangos pabularia Lindl., 1825, Quart. Journ. Sci. 19: 7.

$\equiv$ Cachrys pabularia (Lindl.) Baill., 1879, Hist. P1. (Baillon) 7: 193. 
$\equiv$ Koelzella pabularia (Lindl.) M. Hiroe, 1958, Umbellif. Asia 1: 147.

Type: INDIA. "In Indiae orientalis temperatis circa Draz, Moorcraft".

= Hyalolaena sewerzowii Regel et Herder, 1866, Bull. Soc. Imp. Naturalistes Moscou 39(3): 114.

Type: UZBEKISTAN. "Mittel-Asien (Turkestan) [Trajectus inter Keles et Chirchik, montes Kyrjintau, 4-6000'], 1857, Sewerzow" (holotype LE! [LE01065764-LE01065770]).

= Prangos hissarica Korovin, 1973, Izv. Akad. Nauk Tadzhiksk. SSR, Otd. Estest. Nauk 1(50): 18.

Type: TAJIKISTAN. "Montes Hissarici, vall. fluv. May-Chura, trajectus Schutur-Gardan. 12 VIII 1944. Pissjaukova 559" (holotype TAD!; isotype LE! [LE00051399]).

Distribution in MA \& K: Kazakhstan (S: S Kazakhstan prov., Zhambyl prov.), Kyrgyzstan (Batken prov., Jalal-Abad prov., Naryn prov., Osh prov., Talas prov.), Tajikistan (Kŭhistani Badakhshan, Sughd prov., Khatlon prov., Dushanbe prov.), Uzbekistan (Navoi prov., Dzhizak prov., Samarqand prov., Qashqadarya prov., Surxondarya prov., Toshkent prov., Namangan prov.), Turkmenistan (Lebap prov.).

Distribution in other Asian countries: India, Pakistan, Afghanistan.

Distribution area: Eastern and South-Western Asia.

Prangos pabularia Lindl. subsp. cylindrocarpa (Korovin) Pimenov et V. N. Tikhom. 1981, in S. K. Cherepanov, Sosud. Rast. SSSR: 28.

三 Prangos cylindrocarpa Korovin, 1948, Not. Syst. Herb. Inst. Bot. Zool. Acad. Sci. Uzbekistan 12: 24

Type: UZEKISTAN. "Pamiralaj occidentalis, fontes fl. Yakkobag-darja, prope pagum Taschkurgan, in clivis arduis argillosis schistosisque, 30 VI 1936, Botschantzev \& Butkov 50"1 (holotype TASH! [TASH002241, TASH002242]; isotype TASH! [TASH002243, TASH002244]).

Distribution in MA \& $\mathrm{K}$ : Uzbekistan, Turkmenistan.

Prangos pabularia Lindl. subsp. gyrocarpa (Kuzmina) Lyskov et Pimenov, 2017, Plant Syst. Evol. 303(7): 825.

$\equiv$ Prangos gyrocarpa Kuzmina, 1962, Bot. Zhurn. (Leningrad) 47(2): 253, in adnot.

Type: KYRGYZSTAN. "In declivibus australibus jugi Alaici, vallis Alaicis, basin fl. Taldyk, prope pagum Daraut-Kurgan, 22 IX 1948,
Stanjukovicz 569" (holotype LE! [LE00051401]; isotype LE! [LE00051400]).

Distribution in MA \& K: Kyrgyzstan (Osh prov.). Endemic.

Distribution area: South-Western Asia.

Prangos pabularia Lindl. subsp. lamellata (Korovin) Pimenov et V. N. Tikhom., 1981, in S. K. Cherepanov, Sosud. Rast. SSSR: 28.

$\equiv$ Prangos lamellata Korovin, 1959, in Vvedensky (ed.), Fl. Uzbekist. 4: 490, 323.

Type: UZBEKISTAN. "Pamiro-Alaj occidentalis, jugum Zeravschanicum, prope pagum Urgut, angustis Sailyk, pratulum prope ad confluentes cum affluxio, 06 V 1936, Gnezdillo 167" (holotype TASH! [TASH002247, TASH002248]).

Distribution in MA \& K: Uzbekistan.

Prangos pabularia Lindl. subsp. sarawschanica (Regel et Schmalh.) Lyskov et Pimenov, 2017, Plant Syst. Evol. 303(7): 825.

= Hippomarathrum sarawschanicum Regel et Schmalh., 1878, Trudy Imp. S.-Peterburgsk. Bot. Sada 5(2): 603, in adnota.

= Prangos sarawschanica (Regel et Schmalh.) Korovin, 1948, Not. Syst. Herb. Inst. Bot. Zool. Acad. Sci. Uzbekistan 12: 24.

Type: TAJIKISTAN. "Systema fluminis Sarawschan, pagum Ansob, 7000'. 22 VI 1870. O. A. Fedtschenko" (lectotype LE! [LE00051332], designated by V. M. Vinogradova 2001: 52; isolectotype LE [LE00051331]; "Zarawschan, angustis Dschidschik 19 VI 1870. O. A. Fedtschenko" (syntype LE [LE00051330]).

Prangos pubescens (Pall. ex Spreng.) Pimenov et Kljuykov, 2015, Bot. Zhurn. (St. Petersburg) 100(7): 724 .

$\equiv$ Ferula pubescens Pall. ex Spreng., 1820, in Syst. Veg. ed. 15 bis (Roemer et Schultes) 6: 598.

$\equiv$ Cachrys pubescens (Pall. ex Spreng.) Schischk., 1950, in Schischkin (ed.), Fl. URSS 16: 259.

Type: RUSSIA. "Sibiria. Pallas" (holotype HAL! [HAL0033090]).

Distribution in MA \& K: Kazakhstan (NE: E Kazakhstan prov.; Central: Karagandy prov.)

Distribution in other Asian countries: Russia.

Distribution area: Central Asia.

Prangos tschimganica B. Fedtsch., 1899, Bull. Herb. Boissier 7: 180.

Type: UZBEKISTAN. "Pesochny [sandy] pass prope Tschimgan, 17 VII 1897, O. A. Fedtschenko \& 
B. A. Fedtschenko" (lectotype LE! [LE00051414]; designated here; isolectotypes LE! [LE00051412, LE00051413]).

Distribution in MA \& K: Kazakhstan (S: S Kazakhstan prov.), Kyrgyzstan (Jalal-Abad prov.), Uzbekistan (Toshkent prov.). Endemic.

Distribution area: South-Western Asia.

Psammogeton canescens (DC.) Vatke, 1876, Ind. Sem. Horti Berol. App. 2: 3.

$\equiv$ Athamanta canescens DC., 1830, Prodr. (A. P. de Candolle) 4: 155.

Type: [Syria/Iraq] "Inter Alep et Baghdad, Olivier \& Bruguière".

= Pimpinella crinita Boiss., 1844, Ann. Sci. Nat. sér. 3, 1: 131.

= Psammogeton crinitus (Boiss.) Boiss., 1872, Fl. Orient. (Boissier) 2: 1078.

= Daucus crinitus (Boiss.) Kuntze, 1887, Trudy Imp. S.-Peterburgsk. Bot. Sada 10: 191, nom. illeg. (Art. 53.1), non Desf. (1798).

= Cuminum crinitum Koso-Pol., 1916, Bull. Soc. Imp. Naturalistes Moscou, n. s. 29: 208.

Type: [Iran] "In Persia, Aucher-Eloy 4587" (lectotype G-BOIS!; designated by Pimenov \& Jacquemoud (2020: 81); isolectotypes BM! [BM000944703, BM000944704], FI!, K! [K000695506], P [P00834466, P00834467]).

= Psammogeton biternatus Edgew. var. villosus C. B. Clarke, 1879, in Fl. Brit. India (J. D. Hooker) 2: 719 .

= Psammogeton canescens (DC.) Vatke var. villosus (C. B. Clarke) Raizada, 1976, Suppl. Duthie's Fl. Upper Gangetic Plain 5: 88.

Type: PAKISTAN. "[Baluchistan] Scinde, Stocks 1102" (lectotype K! [K000695501], designated here; isolectotypes K [ K000695502, K000695503]).

= Psammogeton caramanicus Bornm., 1934, Repert. Spec. Nov. Regni Veg. 36: 343.

Type: IRAN. "In collibus regionis calidae pr. Kerman, ca 2000 m, 1892, Bornmüller 3807, 3708" (lectotype B! [B 10 0366018, B 10 0366019]; designated here by Pimenov; isolectotypes E [E00000112, E00000113], G! [G00367246], HBG [HBG513099], HBG513100], JE! [JE00003615, JE00003959], KFTA [KFTA0001964], LD! [LD1245021]).

Distribution in MA \& K: Turkmenistan (Akhal prov., Mary prov.)

Distribution in other Asian countries: India, Pakistan, Afghanistan, Iran, Iraq.

Distribution area: Eastern, South-Western and W Tropical Asia.
Pseudotrachydium dichotomum (Korovin) Pimenov et Kljuykov, 2000, Feddes Repert. 111(78): 527.

$\equiv$ Trachydium dichotomum Korovin, 1948, Not. Syst. Herb. Inst. Bot. Zool. Acad. Sci. Uzbekistan 12: 19.

$\equiv$ Aulacospermum dichotomum (Korovin) K1juykov, Pimenov et V. N. Tikhom., 1976, Byull. Moskovsk. Obshch. Isp. Prir. Otd. Biol. 81(4): 82.

Type: UZBEKISTAN. "Pamir-Alaj; montes Kuhitang supra p. Kyzyl-alma, 28 VI 1927, Popov 193" (holotype TASH! [TASH002238]; isolectotypes E!, LE! [LE00051317]).

= Trachydium depressum (Boiss.) Boiss. subsp. chitralicum Rech. f. et Riedl, 1963, in Biol. Skr. 13(4) (Symb. Afghan. 5): 131.

Type: PAKISTAN. "Chitral, Oihor Gol, BowesLyon 825" (holotype BM! [BM000885511]; isotypes RAW, W [W 1960-0023330]).

Distribution in MA \& K: Kyrgyzstan (Batken prov., Osh prov.), Tajikistan (Kŭhistani Badakhshan, Sughd prov., Dushanbe prov.), Uzbekistan (Samarqand prov., Qashqadarya prov., Surxondarya prov.), Turkmenistan (Lebap prov.).

Distribution in other Asian countries: Pakistan, Afghanistan.

Distribution area: South-Western Asia.

Pseudotrachydium kopetdaghense (Korovin) Sennikov \& Pimenov, comb. nov.

= Trachydium kopetdaghense Korovin, 1924, in Byull. Sredne-Asiatsk. Gosud. Univ. 7: 23.

Type: TURKMENISTAN "Regio Turkomanica, Kopet-Dagh, in cacumime montium Chapan, zona verticalis stepposa. 02 VII 1923. Korovin 423" (lectotype TASH [TASH002231]; designated here by Sennikov \& Pimenov); isolectotypes TASH [TASH002232, TASH002233]).

$=$ Trachydium vesiculoso-alatum Rechinger 1940, Repert. Spec. Nov. Regni Veg. 48: 125, tab. 302, fig. 2. (1940: 125)

= Pseudotrachydium vesiculoso-alatum (Rech. f.) Pimenov \& Kljuykov, 2000, Feddes Repert. 111(7-8): 527.

$\equiv$ Aulacospermum vesiculoso-alatum (Rech. f.) Kljuykov, Pimenov \& V. N. Tikhom., 1976, Byull. Moskovsk. Obsc.Isp. Prir. Otd. Biol. 81(4): 82.

Type: IRAN. "Khorasan: Kuh-e-Bizg, felsige Abhänge der Juniperus polycarpa-Stufe, ca. 2000 $\mathrm{m}, 05$ VII 1937, R. H. Rechinger \& F. Rechinger 1495" (holotype W; isotype BM [BM000944722]). 
Distribution in MA \& K: Turkmenistan (Akhal prov.).

Distribution in other Asian countries: Afghanistan, Iran

Distribution area: South-Western Asia.

Note: The nomenclatural details see in A. N. Sennikov \& M. G. Pimenov, Nomenclatural notes on four species of Umbelliferae (Apiaceae) in Middle Asia. Annales Botanicae fennicae (in press).

Sajanella monstrosa (Willd. ex Spreng.) Soják 1980, Čas. Nár. Mus. Praze, Rada Prrír.148(3-4): 209.

$\equiv$ Athamanta monstrosa Willd. ex Spreng.1820, in Syst. Veg. ed. 15 bis (Roemer et Schultes) 6: 495.

$\equiv$ Libanotis monstrosa (Willd. ex Spreng.) DC., 1829, Col. Mém. 5 (Mém. Fam. Ombell.): 48.

$\equiv$ Sajania monstrosa (Willd. ex Spreng.) Pimenov, 1974, Byull. Moskovsk. Obshch. Isp. Prir. Otd. Biol. 79, 3: 114.

三 Schulzia monstrosa (Willd. ex Spreng.) M. Hiroe, 1979, Umbelliferae World: 760.

Type: RUSSIA. "In Sibiria, Stephan" (holotype B-Willd! [B-W 05730-01 0]).

= Athamanta compacta Ledeb., 1829, Fl. Altaic. (Ledebour) 1: 327.

= Schulzia compacta (Ledeb.) Ledeb., 1844, Fl. Ross. (Ledebour) 2: 258, "Schultzia".

Type: RUSSIA. "In humidiusculis alpium v. gr. ad fontes fl. Tscharysch et alibi [Altai, in humidiusculis alpium], VII-VIII 1826, Ledebour \& Bunge 272" (lectotype LE!; designated by V. M. Vinogradova (2005: 959; isolectotypes C!, K! [K000681258, K000681259]), “Alp. ad Tschuja, Bunge" (syntype LE!).

=Libanotis stephaniana DC., 1830, Prodr. (A. P. de Candolle) 4: 151.

= Athamanta compacta Ledeb. var. laciniata Ledeb., 1829, Fl. Altaic. (Ledebour) 1: 327.

$=$ Schulzia compacta (Ledeb.) Ledeb. var. laciniata Ledeb., 1844, Fl. Ross. (Ledebour) 2: 258.

= Libanotis monstrosa (Willd. ex Spreng.) DC. var. laciniata (Ledeb.) Krylov, 1935, Fl. Zapadnoi Sibiri 8: 2074.

Type: RUSSIA. "[Altai]. In humidiusculis alpium v. gr. ad fontes fl. Tscharysch et alibi. Ledebour". (lectotype LE!; designated here by Pimenov).

Distribution in MA \& K: Kazakhstan (NE: E Kazakhstan prov.).

Distribution in other Asian countries: Russia.

Distribution area: Northern Asia.

Note: The name of Sajania monstrosa is nomen illegitimum as in its description (Pimenov, 1974) in
Sajania Pimenov the earlist homonym of generic name Sajania A. G. Vologdin (1962) (fossil Algae) did not taken into account.

Sanicula europaea L., 1753, Sp. P1.: 235.

$\equiv$ Caucalis sanicula Crantz, 1767, Stirp. Austr.

Fasc. 3: 125.

$\equiv$ Astrantia diapensia Scop., 1771, Fl. Carniol. (ed. 2) 1: 186, nom. illeg. (Art. 52.1).

三 Sanicula sylvatica Salisb., 1796, Prodr. stirp.

Chap. Allerton: 159, nom. illeg. (Art. 52.1).

$\equiv$ Sanicula vulgaris Fr., 1845, Summa Veg. Scand.: 24, nom. illeg. (Art. 52.1).

Type: EUROPE. In Europae sylvis montosis [Sweden Gotland], 333.1 (lectotype LINN [LINNHL333-1], designated by Reduron et Jarvis in Jarvis et al., Regnum Veg. 127: 8 (1993)).

$=$ Sanicula officinalis Gouan, 1762, Hortus Monsp.: 131.

Type: EUROPE, FRANCE. Hab. Monspelii, à l'Esperon, au Capouladou et au bois de Salvous.

= Sanicula officinarum Neck, 1768, Delic. gallobelg.: 137.

= Caucalis capitata Stokes, 1812, Bot. Mat. Med. 2: 64.

Distribution in MA \& K: Kazakhstan (NE: E Kazakhstan prov.).

Distribution in other Asian countries: Russia, Iran, Azerbaidzhan, Georgia, Armenia, Turkey.

Distribution area: Asia (Northern, SouthWestern and Mediterranean), Europa, Africa (N).

Sanicula giraldii H. Wolff, 1913, in Pflanzenr. (Engler) Umbellif.-Sanicul. 61(IV, 228): 60.

Type: CHINA. "Nord-Schensi, südl. Lao-y-san, auf dem Berge Sciu-ian-san, am Kan-y-huo [China interior, provincia Schensi septentr. Monte Sciu-iansan, lungo il fiume Kan-y-huo, a Sud del Lao-y-san], 15 V 1899, Giraldi 5838" [herbarium A. Biondi] (holotype FI! [FI014784]).

= Sanicula uralensis Kleop. ex Kamelin, Czubarov et Shmakov, 2002, Turczaninowia 5, 2: 18, fig. 1, map 3.

Type: EUROPA, RUSSIA. "Provincia Ufa, silvae montanae prope officinam metallurgicam Simsky, 20 VII 1888, Antonov" (holotype LE!).

Distribution in MA \& K: Kazakhstan (NE: E Kazakhstan prov.).

Distribution in other Asian countries: Russia, China.

Distribution area: Asia (Northern, and Eastern), Europa (E). 
Scandix aucheri Boiss., 1844, Ann. Sci. Nat. sér. 3, 2: 58 .

$\equiv$ Scandicium aucheri (Boiss.) Bornm. 1935, Repert. Spec. Nov. Regni Veg. 39: 121.

Type: IRAN. "Persia, prope Ispahan, AucherEloy 4618" (lectotype G-BOIS!; designated here by Pimenov \& Jacquemoud (2020: 36); isolectotypes G! [G00370271, G00370272], P! [P00834418], W [W0054257]).

= Scandix damascena Bornm. 1912, Repert. Spec. Nov. Regni Veg. 10: 468.

$=$ Scandicium stellatum (Banks et Sol.) Thell. var. damascenum (Bornm.) Zohary,1941, Palestine J. Bot. (Jerusalem Ser.) 2: 168.

Type: SYRIA. "In Antilibani (Syriae) declivitatibus orientalibus, in rupestribus aridissimis montis Dshebel Kasioun ditionis urbis Damasci, alt. c. 8-900 m. 13-14 V 1910. J. \& F. Bornmüller 11820" (lectotype B! [B 10 0367208]; designated here; isolectotype B [B 10 0367209]).

Distribution in MA \& K: Turkmenistan (Balkan prov., Akhal prov.)

Distribution in other Asian countries: Pakistan, Afghanistan, Iran, Azerbaidzhan, Armenia, Turkey, Syria.

Distribution area: South-Western Asia.

Scandix pecten-veneris L., 1753, Sp. P1.: 256.

$\equiv$ Chaerophyllum pecten-veneris (L.) Crantz, 1767, Stirp. Austr. Fasc. 3: 66.

$\equiv$ Scandix pecten Scop., 1771, Fl. Carniol. (ed. 2) 1: 211, nom. illeg. (Art. 52.1).

$\equiv$ Myrrhis pecten-veneris (L.) All., 1785, Fl. pedem. 2: 29 .

$\equiv$ Pecten veneris Lam., 1779, Fl. franç. 3: 437.

$\equiv$ Chaerophyllum rostratum Lam., 1785, in Encycl. (Lamarck et al.) 1(2): 685.

$\equiv$ Scandix rostrata (Lam.) Salisb., 1796, Prodr. stirp. Chap. Allerton: 166, nom. illeg. (Art. 52.1).

$\equiv$ Pectinaria vulgaris Bernh., 1800, Syst. Verz. (Bernhardi): 168, nom. illeg. (Art. 52.1).

$\equiv$ Scandix pectiniformis St. Lag., 1880, Ann. Soc. Bot. Lyon 7: 70, nom. illeg. (Art. 52.1).

$\equiv$ Wylia pecten-veneris (L.) Bubani, 1900, Fl. Pyren. (Bubani) 2: 407.

$\equiv$ Selinum pecten (Scop.) E. H. L. Krause, 1904, in Fl. Deutschl. (Sturm), ed. 2 12: 75, fig. 10.

Type: EUROPE. "Inter Germaniae et Europae australioris segetes, 364.2" (lectotype LINN [LINNHL364-2], designated by Jafri in Jafri et El-Gadi (eds.), Fl. Libya 117: 20 (1985)).

= Scandix cretica Mill., 1768, Gard. Dict. (ed. 8): Scandix N 4.
= Scandix pectinifera Stokes, 1812, Bot. Mat. Med. 2: 122.

= Scandix vulgaris S. F. Gray, 1821, Nat. arr. Brit. pl. 2: 503.

$=$ Scandix persica Marloth, 1838, Linnaea (litt.) 12: 87 .

Type: IRAN. E Persia misit hort. Imp. Petropolitanus.

$=$ Scandix pecten-veneris L. var. brevirostris Boiss., 1872, Fl. Orient. (Boissier) 2: 915.

Type: EUROPE, GREECE. "In cultis praesertim montanis et saxosis montes Lassiti et mons Ida Cretae. V 1846. Heldreich 1498" (lectotype G-BOIS!, designated Pimenov \& Jacquemoud (2020: 35); SYRIA. "Syria, Antilibanus. V-VI 1846. Boissier" (syntype G-BOIS!); AZERBAIDZHAN. "Georgia, in vineis prope Helenendorf. VIIVIII 1838. Hohenacker" (syntypes G-BOIS!, $\mathrm{K}$ ! [K000695804], P [P03260589, P03260591]); AFGHANISTAN. "Afghania [Affghanistan]. 1851. Griffith 1026" (syntypes G-BOIS!, K [K000695805, K000695806]); IRAN/PAKISTAN. "Belutschia [Beloutchistan, Zahue]. 1851. Stokes 747" (syntypes G-BOIS!, K! [K000695805, K000695806, K000695808]); EUROPE, GREECE. "Creta, in saxosis Mt. Ida 5000-6000'. VI 1846. Heldreich" (syntype G-BOIS!).

$=$ Scandix iberica M. Bieb. var. parviflora Kuntze, 1887, Trudy Imp. S.-Peterburgsk. Bot. Sada 10: 192.

Type: GEORGIA. "Kachetien, V 1886, Kuntze" (holotype NY [NY004062363]).

$=$ Scandix pecten-veneris L. var. involucrata Bornm, 1905, Mitt. Thuering. Bot. Vereins, n.f. 6: 66.

Type: TURKEY. "Tokat [Anatolia ad Tokat (Wilajet Siwas), 600-700 m, IV 1893], Bornmüller 3347" (holotype JE [JE00002194]).

= Scandix pecten-veneris L. var. glabrescens Koso-Pol., 1920, Izv. Glavn. Bot. Sada R.S.F.S.R. 36(1) (Fl. As. Ross. 15): 57.

Type: not indicated.

= Scandix pecten-veneris L. var. pilosulum KosoPol., 1920, Izv. Glavn. Bot. Sada R.S.F.S.R. 36(1) (Fl. As. Ross. 15): 57.

Type: not indicated.

= Scandix pecten-veneris L. var. longistyla Post, 1932, Fl. Syria, Palestine and Sinai 1: 353.

Type: LEBANON. Beirut, 20 VI 1892, Post 113 (holotype BEI).

$=$ Scandix pecten-veneris L. var. multifida Gombault,1943, Bull. Soc. Bot. France 90(4-6): 55. 
Type: LEBANON. "Jebel Baroouk, vers 1800 mètres sur les crêtes, Blanche 726" (holotype $\mathrm{P}$ [P00723448]; "Maase ech Chouf. 28 V 1933. Gombault 2511" (syntype P [P00602124])

$=$ Scandix pecten-veneris L. var. pleiactinia Bornm. 1945, in Danish Sci. Invest. Iran 4: 14.

Type: IRAN. "Kharon, 1300 m, 1945, Koie $721 "$.

Distribution in MA \& K: Kazakhstan (S: S Kazakhstan prov., Zhambyl prov.), Kyrgyzstan (Batken prov., Jalal-Abad prov., Ysyk-köl prov., Naryn prov., Osh prov., Talas prov.), Tajikistan (Kŭhistani Badakhshan, Sughd prov., Khatlon prov., Dushanbe prov.), Uzbekistan (Navoi prov., Dzhizak prov., Samarqand prov., Qashqadarya prov., Surxondarya prov., Toshkent prov., Andijon prov.), Turkmenistan (Balkan prov., Akhal prov., Lebap prov., Mary prov.).

Distribution in other Asian countries: Russia, India, Pakistan, Afghanistan, Iran, Azerbaidzha, Georgia, Armenia, Turkey, Iraq, Saudi Arabia, UAE, Oman, Lebanon, Syria, Israel, Jordan, Cyprus.

Distribution area: Asia (Northern, SouthWestern and Mediterranean), Europa, Africa, N. America (adv.), Australia \& Oceania (adv.).

Scandix stellata Banks et Sol. 1794, in Nat. Hist. Aleppo (ed. 2) 2: 249.

三Scandicium stellatum (Banks et Sol.) Thell. 1919, Repert. Spec. Nov. Regni Veg. 16: 16.

Type: SYRIA. "Aleppo, sine loco exacto, Russel" (syntypes BM [BM000944714, BM000944715]).

$=$ Scandix pinnatifida Vent. 1800, Descr. P1. Nouv.: tab. 14.

$=$ Chaerophyllum pinnatifidum (Vent.) Poir.1804, in Encycl. (J. Lamarck et al.) 5: 144.

= Birostula pinnatifida (Vent.) Raf. 1840, Good Book: 59.

Type: IRAN. "Sur la route d'Amadan à Tehran, introduite chez Cels (Paris), Bruguière \& Olivier" (lectotype G [G00341470]; designated here; IRAQ. "De Bagdad à Kermanchah, Olivier \& Bruguière" (syntype P [P03260537]).

$=$ Scandix pinnatifida Vent. var. songarica Schrenk, 1841, Enum. Pl. Nov. 1: 62.

Type: KAZAKHSTAN. "Semina lecta versus montes Labassy. 14 VI 1840, Schrenk" (holotype LE?).

$=$ Scandix pinnatifida Vent. var. hirsuta K. Koch, 1842, Linnaea 16: 363.

Type: GEORGIA. "In Georgia prope Tiflis et in Armenia, K. Koch".
= Scandix russeliana Griseb., 1843, Spic. Fl. Rumel. 1: 369.

Type: IRAQ. "In Mesopotamia, Donietti" (holotype GOET [GOET011410]).

= Scandix hispidula Bertol. 1842, Misc. Bot. (Bertoloni) 5: 427.

Type: IRAQ. "Ex vallo castri Sedjim Kala. Chesney".

= Scandix pinnatifida Vent. var. decipiens Bornm. 1898, Verh. Zool.-Bot. Ges. Wien 48: 593.

Type: LEBANON. "Libanon: In der Alpenregion des Sannin bei 2300 m, am Rand der Schneefelder zwischen Felsen zusammen mit Lamium Ehrenbergii Boiss., Bornmüller 11823" (syntype JE [JE00020230]).

= Scandix fedtschenkoana Koso-Pol., 1916, Izv. Imp. S.-Peterburgsk. Bot. Sada 16: 227.

Type: TURKMENISTAN. "In regione transcaspica, in montibus Bolchie Balchany, prope Djebel. 20 IV 1912. Andrjusczenko" (holotype LE! [LE00051027]).

= Scandix manjurkiana Tamamsch., 1932, Bot. Arch. 34: 524.

Type: ARMENIA. "In Armenien, Nahe von Erivan zwischen Djervesch und Woachaberd, 13 VI 1931, Tamamschjan" (holotype ERE?).

= Scandicium stellatum (Banks et Sol.) Thell. var. eriophorum Bornm. 1945, in Danish Sci. Invest. Iran 4: 14.

Type: IRAN. "Kechwar, 700-900 m, Koie 888".

Distribution in MA \& K: Kazakhstan (W: Mangystau prov.; S: S Kazakhstan prov., Zhambyl prov.; SE: Almaty prov.), Kyrgyzstan (Batken prov., Jalal-Abad prov., Ysyk-köl prov., Osh prov., Talas prov., Chüh prov.), Tajikistan (Kŭhistani Badakhshan, Sughd prov., Khatlon prov., Dushanbe prov.), Uzbekistan (Kara-kalpak, Navoi prov., Dzhizak prov., Samarqand prov., Qashqadarya prov., Surxondarya prov., Toshkent prov., Namangan prov., Fergona prov., Andijon prov.), Turkmenistan (Balkan prov., Akhal prov., Lebap prov., Mary prov.)).

Distribution in other Asian countries: Russia, China, India, Pakistan, Afghanistan, Iran, Azerbaidzhan, Gejrgia, Armenia, Turkey, Iraq, Saudi Arabia, Yemen, Lebanon, Syria, Israel. Jordan, Egypt (Sinai), Cyprus.

Distribution area: Asia (Northern, Central, South-Western and Mediterranean), Europa, Africa (N).

Schrenkia alaica Pimenov, 2015, Phytotaxa 195(4): 264, figs. 8, 9. 
Type: KYRGYZSTAN. "Provincia Osch, jugum Alaicum, declivum septentrionale, in valle fluminis Ak-bura, inter ostio fluvii Schtschjut et ostio fluvii Kaindy, $40^{\circ} 08^{\prime}$ N, $72^{\circ} 58^{\prime}$ E. 17 VII 1981. Pimenov, Vasilieva \& Lavrova 503" (holotype MW! [MW0595633], isotype LE [LE00051034]).

Distribution in MA \& K: Kyrgyzstan (Osch prov.). Endemic.

Distribution area: South-Western Asia.

Schrenkia congesta Korovin, 1962, Trudy Inst. Bot. Akad. Nauk Kazakh. SSR 13: 245.

Type: KAZAKHSTAN. "Prov. Syr-Darya, distr. Chimkent, montes Talassky Alatau, in valle Dschebagly-su, ad declivitatem lapidosam, 18 VIII 1921, Abolin \& Popov 8365" (lectotype TASH! [TASH002173]; designated here; isolectotype TASH [TASH002172]).

Distribution in MA \& K: Kazakhstan (S: S Kazakhstan prov., Zhambyl prov.). Endemic.

Distribution area: South-Western Asia.

Schrenkia fasciculata Korovin, 1948, Not. Syst. Herb. Inst. Bot. \& Zool. Acad. Sci. Uzbekistan 12: 15. 1948.

References: Korovin 1959a: 291.

Type: UZBEKISTAN. "Montes Nuratau, in regione urbis Dshizak, promont. Kojtasch [Bagadan]. 25 V 1914. Michelson" (holotype LE! [LE00051037]; isotypes LE [LE00051038-LE051040]).

Distribution in MA \& K: Tajikistan (Sughd d.), Uzbekistan (Navoi d., Samarqand d.). Endemic.

Distribution area: South-Western Asia.

Schrenkia golickeana (Regel et Schmalh.) B. Fedtsch., 1909, Consp. Fl. Turkest. (O. A. Fedtschenko \& B. A. Fedtschenko) 3: 125. 1909.

$\equiv$ Daucus golickeanus Regel et Schmalh., 1877, Trudy Imp. S.-Peterburgsk. Bot. Sada 5(2): 599. 1877.

$\equiv$ Anidrum golickeanum (Regel et Schmalh.) Koso-Pol., 1916, Bull. Soc. Imp. Naturalistes Moscou, n. s. 29: 147.

$\equiv$ Bifora golickeana (Regel et Schmalh.) KosoPol., 1916, Bull. Soc. Imp. Naturalistes Moscou, n. s. 29: 147. 1916, in adnot.

Type: KAZAKHSTAN. "In fluvium Syr-Darja, 1871, Golike" (holotype LE [LE00052233]

= Schrenkia syrdarjensis Lipsky, 1904, Trudy Imp. S.-Peterburgsk. Bot. Sada 23: 160, p. p.

Type: KAZAKHSTAN. "Regio Syrdajensis, Aksu. 05 VI 1907. Lipsky 267" (lectotype LE! [LE00051187], designated by V. M. Vinogradova (1997b: 58). This specimen did not listed in a protologue!; "Pr. Tulkubasch \& Jaskilschin ad fonted fl. Aksu, inter Aulie-Ata \& Tschimkent. V 1880, Mussa" (syntypes LE [LE00051188-LE00051191]; "inter Chodschikent et Mt. Tschimgan. 11 VI 1881. A. Regel" (syntype LE [LE00051192, LE00051193]; "Niaz-beck 30 V 1903, Lipsky" (syntype LE [LE00051194]; "Alexandrovski Mts, Kaschkasu, 09 VII 1903. Fischikov" (syntype LE [LE00051195]; "Naldybulek (Angren) V 1880. A. Regel" (syntype LE [LE00051182-LE00051184]; "Ala-archa 6-7000'. V 1913, Fetissov" (syntype LE [LE00051185]).

= Anidrum minkwitziae Koso-Pol., 1920, Izv. Glavn. Bot. Sada R.S.F.S.R. 36(1) (Fl. As. Ross. 15): 85 .

Type: KAZAKHSTAN. "Syr-Darja prov., AulieAta distr., angustis Berk-kara (Karatau), 23 V 1909, Minkwitz 29" (lectotype LE! [LE00051201], designated by V. M. Vinogradova (1999: 82)); isolectotypes LE (LE00051202, LE00051203]; "Aulie-Ata distr. Angustis Kok-tal, 1909, Minkwitz 388” (syntype LE [LE00051204).

= Anidrum roschewitzii Koso-Pol., 1920, Izv. Glavn. Bot. Sada R.S.F.S.R. 36(1) (Fl. As. Ross. 15): 85 .

Type: KIRGHYZSTAN. "Tian-Schan centaralis, Semirechie prov, Pischpek distr., vallis fl. Susamyr. 24 VII 1908. Roschewitz 1424" (holotype LE! [LE00051205]; isotype LE! [LE00052061]; "Angust. Ala-artcha, m. Alexander, 6-7000". V 1913. Fetissov" (syntype LE [LE00051208]).

= Anidrum subinvolucratum Koso-Pol., 1920, Izv. Glavn. Bot. Sada R.S.F.S.R. 36(1) (Fl. As. Ross. 15): 86

Type: KIRGHYZSTAN. "Tian-Schan centralis, steppa prope st. Ak-Chulak, 10 V 1908, Roschewitz" (lectotype LE! [LE00051207], designated by V. M. Vinogradova (1999: 82)).

Distribution in MA \& K: Kazakhstan (Central: Kzyl-Orda prov.; S: S Kazakhstan prov., Zhambyl prov.), Kyrgyzstan (Batken prov., Naryn prov., Osh prov., Chüh prov.), Tajikistan (Sughd prov.), Uzbekistan (Dzhizak prov., Toshkent prov.). Endemic.

Distribution area: South-Western Asia.

Schrenkia hebecarpa (Pimenov et Kamelin) Pimenov, 1915, Phytotaxa 195(4): 264.

$\equiv$ Kosopoljanskia hebecarpa Pimenov et Kamelin, 2002, Bot. Zhurn. (St. Petersburg) 87(7): 110. 
Type: KYRGYZSTAN. "Kyrgyzstan, jugum Alaicum, declivum septentrionale, in valle fluvii Isfairam-sai, inter locum Dara et ostium rivulum Schibbe, in schistosis lapidosis, 12 VII 1987, Pimenov, Kljuykov, Vassilieva \& Lavrova 299" (holotype LE! [LE00051196]; isotypes MW! [MW0593044MW0593047]).

Distribution in MA \& $\mathbf{K}$ : Kyrgyzstan (Osh prov.). Endemic.

Distribution area: South-Western Asia.

Schrenkia involucrata Regel et Schmalh., 1877, Trudy Imp. S.-Peterburgsk. Bot. Sada 5(2): 606.

ESchrenkia songarica Lipsky, 1904, Trudy Imp. S.-Peterburgsk. Bot. Sada 23: 160, nom illeg. (Art. 52.1).

三 Anidrum involucratum (Regel et Schmalh.) Koso-Pol., 1920, Izv. Glavn. Bot. Sada R.S.F.S.R. 36(1) (F1. As. Ross. 15): 83.

E Bifora involucrata (Regel et Schmalh.) KosoPol., 1920, Izv. Glavn. Bot. Sada R.S.F.S.R. 36(1) (Fl. As. Ross. 15): 83, in adnota.

Type: KAZAKHSTAN. "Songaria, Balchasch, 16 VI 1843, Schrenk 352" (lectotype LE! [LE00051048], designated by V. M. Vinogradova (1997b: 58); isolectotypes BM! [BM000885367, BM000885453], JE! [JE00002669], LE [LE00051049-LE00051058, LE00051060, LE00051061], MANCH!); "Songarei, Chantau, 25 VI 1843, Schrenk 354" (syntype LE!); "Songaria, in collibus ad ac. Balchasch nec non in mont. Bektau-Ata 03-18 VI1843, Schrenk 354"' (syntype LE! [LE00051058, LE00051059]).

Distribution in MA \& K: Kazakhstan (Central: Karagandy prov.; S: Zhambyl prov.). Endemic.

Distribution area: Central Asia.

Schrenkia kultiassovii Korovin, 1924, Byull. Sredne-Asiatsk. Gosud. Univ. 7: 22.1924. [Sched. Herb. Fl. As. Med. 2: N 28 ].

三 Bifora kultiassovii (Korovin) M. Hiroe, 1979, Umbelliferae World: 1130, fig. 190.

Type: KAZAKHSTAN. "Prov. Syr-Darja, distr. Tschimkent. Tian-Schan occidentalis: in montibus siccis lapidosis Duany-tau, 24 VII 1922, Kultiassov 28" (lectotype LE! [LE00051066]; designated by Schischkin (1950: 198); isolectotypes BK, BR! [BR0000005624300], BRNU [BRNU131487], C! [C10008550], E! [E00284195], G! [G00367274, G00367302], K! [K000697377], LE [LE00051066LE00051068, LE00051198], MA [MA85846], MHA!, MO [MO-345350], MW! [MW0593748], NY [NY00406264], P [P00834615], S!, TASH! [TASH002174], W [W 1927 0011380].
Distribution in MA \& K: Kazakhstan (S: S Kazakhstan prov.). Endemic.

Distribution area: South-Western Asia.

Note: A case is similar to that with Angelica tschimganica. S. kultiassovii was described on the basis of one series of duplicates, published as exciccates in Schedae (HFAM 2: no. 28). Korovin in the protologue did not indicate which specimen and in which herbarium is the lectotype (type) of the species name. This was done by Schischkin (1950: 198).

Schrenkia papillaris Regel et Schmalh., 1877, Trudy Imp. S.-Peterburgsk. Bot. Sada 5(2): 608.

三Anidrum papillare (Regel et Schmalh.) KosoPol., 1916, Bull. Soc. Imp. Naturalistes Moscou, n. s. 29: 147.

E Bifora papillaris (Regel et Schmalh.) KosoPol., 1916, Bull. Soc. Imp. Naturalistes Moscou, n. s. 29: 147, in adnot.

三 Hippomarathrum papillare (Regel et Schmalh.) M. Hiroe, 1979, Umbelliferae World: 1225, "papillarum".

Type: KAZAKHSTAN. "In Turkestaniae montibus Karatavicis prope Boroldai, 03 VI 1876, A. Regel 135" (lectotype LE! [LE00051178], designated by V. M. Vinogradova (1997b: 58); isolectotypes K! [K000697378], LE [LE00051176, LE00051177], P [P02530560]).

Distribution in MA \& K: Kazakhstan ( $\mathrm{S}$ : S Kazakhstan prov.). Endemic.

Distribution area: South-Western Asia.

Schrenkia pulverulenta Pimenov, 1983, in Vvedensky (ed.), Opred. Rast. Sred. Azii 7: 370, 191.

Type: KYRGYZSTAN. "Kirghisia, Osch prov., Yangibasar distr., valle fl. Chatkal, riv. Ajakterek, ad declivitatios terrasis, 16 V 1976, Pimenov 750" (holotype MW! [MW0593751]; isotypes LE! [LE00051076, LE00051077, LE00051078]).

Distribution in MA \& K: Kyrgyzstan (JalalAbad prov., Naryn prov., Talas prov., Chüh prov.). Endemic.

Distribution area: South-Western Asia.

Schrenkia pungens Regel et Schmalh., 1877, Trudy Imp. S.-Peterburgsk. Bot. Sada 5(2): 607.

三 Anidrum pungens (Regel et Schmalh.) KosoPol., 1916, Bull. Soc. Imp. Naturalistes Moscou, n. s. 29: 147.

三 Bifora pungens (Regel et Schmalh.) Koso-Pol., 1916, Bull. Soc. Imp. Naturalistes Moscou, n. s. 29: 147 , in adnot. 
$\equiv$ Anidrum vaginatum (Ledeb.) Koso-Pol. var. pungens (Regel et Schmalh.) Koso-Pol., 1920, Izv. Glavn. Bot. Sada R.S.F.S.R. 36(1) (Fl. As. Ross. 15): 82 .

Type: KAZAKHSTAN. "Turkestan, Kulanscheku, Karatau, 1876, A. Regel" (lectotype LE! [LE00051179], designated by V. M. Vinogradova (1997b: 58); isolectotype LE [LE00051180]).

- Schrenkia golickeana auct. non B. Fedtsch.: Schischk., 1950, in Schischkin (ed.), Fl. URSS 16: 195, p. p.

- Schrenkia involucrata auct. non Regel et Schmalh.: Korovin, 1963, in Pavlov (ed.), Fl. Kazakhst. 6: 284, p. p.

Distribution in MA \& K: Kazakhstan (Central: Karagandy prov.; S: S Kazakhstan prov., Zhambyl prov.), Kyrgyzstan (Talas prov.), Uzbekistan (Toshkent prov.). Endemic.

Distribution area: Central and South-Western Asia.

Schrenkia turkestanica (Korovin) Pimenov, 2015, Phytotaxa 195(4): 265.

$\equiv$ Kosopoljanskia turkestanica Korovin, 1923, Trudy Turkestansk. Naučn. Obshch. 1: 85; et 1925, ByullPochvov. Geobot. Sredneas. Gosud. Univ. 1: 19.

Type: KAZAKHSTAN. "Distr. Aulie-Ata, Mt. Ak-tasch prope hibernaculum Kydyr-Ali, ad schistis, 12 VII 1922, Korovin 1427" (lectotype LE [LE00051198], designated by V.Vinogradova (2001: 52); isolectotypes MHA!, TASH (1431，1432,1433,1434, 1435 [TASH003704TASH003706, TASH003710, TASH003711]); "Declivum boreale jugi Talas Alatau, cursus medium fl. Kumysch-tag, prope ostium fl. Taldy-bulak, declivia schistosa. 08 VIII 1922. Korovin 2176" (syntype TASH [TASH002183]); "Conglomerata tertiaria ad ripam dextram fluminis Karagoin, prope ostium hujis. 01 VIII 1922. Korovin 2026, 2027, 2029, 2030" (syntypes LE [LE00051199], TASH [TASH002180-TASH002182]; "Syr-Darya prov., distr. Aulie-Ata, Talas Alatau, riv. Kurkursu-Arabik. 04 IX 1921 Abolin \& Popov 8743" (syntypes TASH [TASH003707-TASH003709].

Distribution in MA \& K: Kazakhstan (S: S Kazakhstan prov., Zhambyl prov.), Kyrgyzstan (Talas prov.). Endemic.

Distribution area: South-Western Asia.

Schrenkia ugamica Korovin, 1948, Not. Syst. Herb. Inst. Bot. Zool. Acad. Sci. Uzbekistan 12: 14.

Type: UZBEKISTAN. "In valle fl. Ugam, Ming-bulak, 16 VIII 1922, Simonova \& Batueva" (holotype TASH! [TASH002175]; isotype MW! [MW0593752]).

Distribution in MA \& K: Kazakhstan (S: S Kazakhstan prov.), Kyrgyzstan (Jalal-Abad prov.), Uzbekistan (Toshkent prov.). Endemic.

Distribution area: South-Western Asia.

Schrenkia vaginata (Ledeb.) Fisch. et C. A. Mey. 1841, in Schrenk, Enum. Pl. Nov. 1: 64.

$\equiv$ Cachrys vaginata Ledeb., 1829, Fl. Altaic. (Ledebour) 1: 366.

$\equiv$ Anidrum vaginatum (Ledeb.) Koso-Pol., 1916, Bull. Soc. Imp. Naturalistes Moscou, n. s. 29: 146.

$\equiv$ Bifora vaginata (Ledeb.) Koso-Pol., 1916, Bull. Soc. Imp. Naturalistes Moscou, n. s. 29: 147, in adnot.

Type: KAZAKHSTAN/RUSSIA. [Altai] "In montibus Dolenkara [deserti Soongoro-Kirghisici] nec non versus lacum Balchasch, 06 VI 1826, Meyer 229" (lectotype LE! [LE00051374]; designated here by Pimenov; isolectotypes C [C10008551], $\mathrm{H}$ [H1393929], HAL [HAL0098496], LE [LE00051375-LE00051380], P [P02790436], PH [PH00010105]).

$=$ Schrenkia vaginata (Ledeb.) Fisch. et C. A. Mey. var. transitoria Lipsky, 1904, Trudy Imp. S.Peterburgsk. Bot. Sada 23: 160.

= Anidrum transitorium (Lipsky) Koso-Pol., 1920, Izv. Glavn. Bot. Sada R.S.F.S.R. 36(1) (Fl. As. Ross. 15): 85.

$=$ Schrenkia transitoria (Lipsky) Kamelin, 1973, Bot. Zhurn. (Leningrad) 58(5): 630, quoad nomen, cum basyon. auct. Koso-Pol.

Type: TAJIKISTAN. "Jagnob, Margif, 18 VII 1892, Komarov" (holotype LE! \{LE00051209]; isotype LE [LE00051210]).

= Schrenkia mogoltavica O. E. Politova, 1978, Byull. Moskovsk. Obshch. Isp. Prir. Otd. Biol. 83(4): 140.

Type: TAJIKISTAN. "Turkestan, prov. Samarkand, montes Mogol-Tau prope pagum Chilkovo, 04 VI 1916, Androssov 1790" (holotype LE! [LE00051069]; isotype LE [LE00051075]).

Distribution in MA \& K: Kazakhstan (NE: E Kazakhstan prov.; S: S Kazakhstan prov., Zhambyl prov.; SE: Almaty prov.), Kyrgyzstan (Batken prov., Jalal-Abad prov., Ysyk-köl prov., Naryn prov., Osh prov., Talas prov., Chüh prov.), Tajikistan (Sughd prov., Dushanbe prov.), Uzbekistan (Dzhizak prov., Navoi prov., Samarqand prov., Toshkent prov., Namangan prov., Fergona prov.).

Distribution in other Asian countries: China.

Distribution area: Central and South-Western Asia. 
Schtschurowskia margaritae Korovin, 1925, Bull. Inst. Pedol. Geobot. Univ. As. Centr. 1: 109.

Type: KAZAKHSTAN. "Kazakh SSR, provincia Syr-Darya, districtus Aulie-Ata, montes Karatau, pars australis, in systemate fluminis Asa, planities Karatau, declivia schistosa angustis Berkara. 20 VI 1925. Sovetkina 514" (lectotype TASH! [TASH002581], designated here; isolectotype TASH [TASH002582]).

Distribution in MA \& K: Kazakhstan (S: Zhambyl prov.). Endemic.

Distribution area: South-Western Asia.

Note: Closely related to $S$. meifolia. In its protologue Korovin (1925) cited one specimen, which evidently is the type or lectotype, located in Tashkent (for the second species, described in the same article, this is rightly noted, but for $S$. margaritae not).

Note: Closely related to $S$. meifolia.

Schtschurowskia meifolia Regel et Schmalh., 1882, Izv. Obshch. Ljubit. Estestv. 34(2) (Descr. P1. Nov. Rar. Fedtsch.): 40.

Type: TAJIKISTAN. "In Kokania valle fluvii Sarawschan in angustis Makschewat, 18 VI 1870, O. A. Fedtschenko" (holotype LE! [LE00052239]), designated by V. M. Vinogradova (1997b: 59)).

Distribution in MA \& K: Kazakhstan (S: S Kazakhstan prov., Zhambyl prov.), Kyrgyzstan (Jalal-Abad prov., Naryn prov., Talas prov.), Tajikistan (Sughd prov., Dushanbe prov.), Uzbekistan (Dzhizak prov., Samarqand prov., Qashqadarya prov., Surxondarya prov., Toshkent prov.). Endemic.

Distribution area: South-Western Asia.

Schulzia albiflora (Kar. et Kir.) Popov, 1940, F1. Alma-Atinsk. Zapovedn.: 35.

三Chamaesciadium albiflorum Kar. et Kir., 1842, Bull. Soc. Imp. Naturalistes Moscou 15: 360.

ESchulzia crinita (Pall.) Spreng. subsp. albiflora (Kar. et Kir.) Pimenov, 1983, in Vvedensky (ed.), Opred. Rast. Sred. Azii 7: 263. 1983.

Type: KAZAKHSTAN. "In herbidis subalpinis et alpinis Alatau ad fl. Sarchan, VII 1841, Karelin \& Kirilov 1514" (lectotype MW! [MW0593896], designated by Gubanov (1993); isolectotypes BM! [BM000793391], BR [BR0000005419586], G [G00367262, G003677277], H [H1395759], K! [K000695716, K000695717, K000695936], LE! [LE00051669-LE00051678], M! [M01722898], NY [NY00405795], P! [P03212454, P03212456], TK, WU! [WU0025941]).
= Chamaesciadium albiflorum Kar. et Kir. var. subacaulis Trautv. 1866, Bull. Soc. Imp. Naturalistes Moscou 39(2): 317.

Type: KAZAKHSTAN. "In montibus Alatau, VII 1841, Schrenk" (lectotype LE!; designated by Pimenov, 2017, in Turczaninowia 20, 2: 207; isolectotype BM!); "In pylis Kokatau, 06 VII 1841, Schrenk" (syntype LE); "In alpis ad fl. Baskan, 29 VII 1841, Schrenk" (syntype LE); "In montibus Dschill-Karagai, 20 VI 1841, Schrenk” (syntype LE).

= Chamaesciadium albiflorum Kar. et Kir. var. caulescens Trautv., 1866, Bull. Soc. Imp. Naturalistes Moscou 39(2): 317.

Type: KAZAKHSTAN. "In excursione versus montes Saratau. VII 1841. Schrenk" (holotype LE).

= Ligusticum baltistanicum H. Wolff, 1930, Repert. Spec. Nov. Regni Veg. 27: 310.

Type: INDIA. "Baltistan, Karpuchu Valley, 13-14000 ft. 10 VII 1892. Duthie 11956" (lectotype K! [K000695935]: designated by Pimenov, 2017, in Turczaninowia 20, 2: 207; isolectotypes BM, E!, L); "Baltistan, Nar nullah, above Dras., 12-13000 ft. 03 VII 1892. Duthie 11794" (syntype $\mathrm{K}$ ! [K000695936]).

= Schulzia tianschanica Lazkov et Kljuykov, 2019, Wulfenia 26: 72.

Type: KYRGIZSTAN. "Santalash gorge, basin of Santalash river, accent to traj. Kumbel, limestone rocks, $2613 \mathrm{~m}, 41^{\circ} 58^{\prime} 02,5^{\prime \prime} \mathrm{N}, 71^{\circ} 08^{\prime} 55,6^{\prime \prime} \mathrm{E} .27 \mathrm{VII}$ 2018. Lazkov" (holotype LE!).

- Schulzia dissecta auct. non C. Norman: Nasir, 1972, in F1. W Pakistan 20: 113, fig. 33, A-C.

Distribution in MA \& $\mathbf{K}$ : Kazakhstan (SE: Almaty prov.), Kyrgyzstan (Jalal-Abad prov., Ysykköl prov., Naryn prov., Osh prov., Talas prov., Chüh prov.), Tajikistan (Kŭhistani Badakhshan, Sughd prov.), Uzbekistan (Toshkent prov.).

Distribution in other Asian countries: China, India, Pakistan.

Distribution area: Central and South-Western Asia.

Schulzia crinita (Pall.) Spreng., 1813, Neue Schr. Naturf. Ges. Halle 2, 1 (P1. Umbell. Prodr.): 30 .

ESison crinitum Pall., 1779, Acta Acad. Sci. Imp. Petrop. 2: 250, tab. 7.

$\equiv$ Sium crinitum (Pall.) Poir., 1811, in Encycl. (Lamarck et al.) Suppl. 1(2): 622.

$\equiv$ Athamanta crinita (Pall.) Ledeb., 1829, Fl. Altaic. (Ledebour) 1: 326. 
$\equiv$ Carum crinitum (Pall.) Koso-Pol., 1916, Bull. Soc. Imp. Naturalistes Moscou, n. s. 29: 198.

Type: RUSSIA. "Altai. Sibiria occidentalis, in udis summarum alpinum. 1829. Ledebour \& Bunge 272" (lectotype LE; designated by V. M. Vinogradova (2005: 959).

Type: RUSSIA. In nivalibus montium Altaicorum ingis, viginti circiter stadia a fortalitia et rivo Tigerek versus occidentalem. [In alpibus altaicis], 1791, Hb. Pallas [Patrin?] (lectotype K!; designated by Pimenov, in Pimenov et Ostroumova, 2012: 210; isolectotypes B-Willd! [B-W 0586103 0, B-W 05861-04 0], BM! [BM000885442], HAL! [HAL0098515], P! [P00228476, P03224725, P03224736]).

Distribution in MA \& K: Kazakhstan (NE: E Kazakhstan prov.).

Distribution in other Asian countries: Russia, China, Mongolia.

Distribution area: Northern Asia.

Schulzia prostrata Pimenov et Kljuykov, 1990, Bot. Zhurn. (Leningrad) 75(1): 94, fig. 5, 6.

Type: KYRGYZSTAN. "Kyrgyzstan, regio Naryn, distr, At-baschy, Tian-Schan Centralis, in systemate fluminis Aksai, vall. fl. Terek, in glareosis inundatis, $\mathrm{h}=3500 \mathrm{~m}, 08$ VIII 1987, Pimenov \& Kljuykov 790" (holotype LE! [LE01066087]; isotypes FRU!, LE [LE01066084, LE01066087], MW! [MW0593893, MW0593897, MW0593899]).

- Trachydium tianschanicum auct. non Korovin: $\mathrm{Pu}$ Fating in Fl. Reipubl. Popularis Sin. 55(1): 203, tab. 107, fig. 1-8. 1979.

Distribution in MA \& K: Kyrgyzstan (Naryn prov.).

Distribution in other Asian countries: China.

Distribution area: Central Asia.

Sclerotiaria pentaceros (Korovin) Korovin, 1952, Trudy Inst. Bot. Akad. Nauk Kazakh. SSR 13: 243. 1962, in nota.

$\equiv$ Kosopoljanskia pentaceros Korovin, 1925, Bull. Inst. Pedol. \& Geobot. Univ. As. Centr. 1: 105, fig. 1.

$\equiv$ Schtschurowskia pentaceros (Korovin) Schischk., 1950, in Schischkin (ed.), Fl. URSS 16: 188.

Type: KYRGYZSTAN. "Tianschan Centralis, jugum Alexandri, trajectus Kainar, ad declivum schistosum, zona subalpina, 18 VIII 1925, Sovetkina 1554" (lectotype TASH! [TASH002178]; designated by Schischkin (1950: 188); isolectotypes LE! [LE00051197, LE00051198], TASH [TASH002176, TASH002177]).
Distribution in MA \& K: Kazakhstan (S: Zhambyl prov.), Kyrgyzstan (Talas prov.). Endemic.

Distribution area: South-Western Asia.

Semenovia alaica Lazkov, 2007, Bot. Zhurn. (St. Perersburg) 92(6): 913.

Type: KYRGYZSTAN. "Jugum Alaicum, declivum generale boreale ad occidentem ab lacum Kurban-Kul, 15 VI 2006, Lazkov" (holotype LE!; isotypes FRU, MW! [MW0594096, MW0594097]).

Distribution in MA \& K: Kyrgyzstan (Batken prov.). Endemic.

Distribution area: South-Western Asia.

Semenovia bucharica (Schischk.) Manden. 1959, Trudy Tbilis. Bot. Inst. 20: 22.

三 Platytaenia bucharica Schischk., 1951, in Schischkin (ed.), Fl. URSS 17: 356, 271.

三Zosima bucharica (Schischk.) M. Hiroe, 1979, Umbelliferae World: 1761.

Type: TAJIKISTAN. "Asia Media, Tajikistania, Jakkabag, 19 V 1891, Lipsky" (holotype LE! [LE00052224]; isotypes LE [LE00052222, LE00052223]).

$=$ Seseli tragioides Pimenov, 2008, Novit. Syst. Pl. Vasc. 40: 190.

Type: TAJIKISTAN. "Tajikistania, jugum Karateginum, declivum septentrinale montibus Kabud-Krym, in systemate fluminis Komarou, in vicinitate pagi Dara-i Tanchak, vallis torrentem Pandara (Pagandara), 39 $09^{\prime} \mathrm{N}, 7^{\circ} 24^{\prime} \mathrm{E} .03 \mathrm{VII}$ 1988. Pimenov, Vasilieva \& Lavrova 244" (MW! [MW0593889], isotypes LE! [LE01066032], MW [MW0593888]).

Distribution in MA \& K: Tajikistan (Sughd prov., Dushanbe prov.), Uzbekistan (Qashqadarya prov., Surxondarya prov.). Endemic.

Distribution area: South-Western Asia.

Semenovia dasycarpa (Regel et Schmalh.) Korovin, 1981, in S. K. Cherepanov, Sosud. Rast. SSSR: 29. 1981.

E Pastinaca dasycarpa Regel et Schmalh., 1977, Trudy Imp. S.-Peterburgsk. Bot. Sada 5(2): 598.

$\equiv$ Zosima dasycarpa (Regel et Schmalh.) Korovin, 1924, Bot. Mater. Gerb. Glavn. Bot. Sada R.S.F.S.R 5, 6: 82.

$\equiv$ Malabaila dasycarpa (Regel et Schmalh.) Schischk., 1951, in Schischkin (ed.), Fl. URSS 17: 262.

$\equiv$ Platytaenia dasycarpa (Regel et Schmalh.) Korovin, 1959, in Vvedensky (ed.), Fl. Uzbekist. 4: 462. 
Type: KYRGYZSTAN. "In Turkestaniae montibus Alexander in trajectu Karabura, 8000' alt., A. Regel" (holotype LE! [LE00052191]).

= Heracleum brignoliifolium Franch., 1883, Ann. Sci. Nat. (sér. 6) 16: 297.

Type: UZBEKISTAN. "Tourpag Bell, vallée de l'Ona Oulgane, dans les monts Tschirtchik, alt. $2300 \mathrm{~m}, 23$ VIII 1881, Capus 530" (lectotype P! [P00753148]; designated by Pimenov, 2017, in Turczaninowia 20, 2: 208; isolectotype LE! [LE00052225] (fragment), P [P00753149, P00753150].

= Platytaenia komarovii Schischk., 1951, in Schischkin (ed.), F1. URSS 17: 357, 272.

= Semenovia komarovii (Schischk.) Manden., 1959, Trudy Tbilis. Bot. Inst. 20: 19.

= Zosima komarovii (Schischk.) M. Hiroe, 1979, Umbelliferae World: 1762.

Type: TAJIKISTAN. "Asia Media, in jugo inter fl. Muran [Churan] et Ksyl-su, in declivio occidentali montis Karimdzag [Karamanak]. 27 VII 1932. Gonczarov, Grigoriev \& Nikitin 809" (lectotype LE! [LE00052228], designated by R. V. Kamelin (Vinogradova, 1997b: 56); isotype LE [LE00052229]).

= Platytaenia rosea Nikitina, 1959, in Vvedensky (ed.), Fl. Kirgiz. SSR 8: 111, nom. inval.; id. ibid., 1960, 9: 207.

Type: KYRGYZSTAN. "Fergana Mts., declivum australe, loc. Kurmaidan. 26 VI 1956. Lebedeva" (holotype FRU!).

= Heracleum multibracteatum Rech. f., 1963, in Biol. Skr. 13(4) (Symb. Afghan. 5): 83.

Type: AFGHANISTAN. "NE: Nuristan: Minjan: Ptili, 2700 m, 19 VII 1948, Edelberg 2128”, (lectotype W! [W 1963-0000592]; designated here by Pimenov; isolectotype C! [C10008553]).

Distribution in MA \& K: Kazakhstan (S: S Kazakhstan prov., Zhambyl prov.), Kyrgyzstan (Batken prov., Jalal-Abad prov., Osh prov., Talas prov., Chüh prov.), Tajikistan (Kŭhistani Badakhshan, Sughd prov., Khatlon prov., Dushanbe prov.), Uzbekistan (Dzhizak prov., Qashqadarya prov., Toshkent prov.).

Distribution in other Asian countries: China, Afghanistan

Distribution area: South-Western Asia.

Semenovia dissectifolia Ukrainskaja et Kljuykov, 2013, Nordic J. Bot. 31(6): 649, Fig. 2, 5-7.

Type: TAJIKISTAN. "Tadshikistania, Badachschan region autonoma, Schugnan districtus, in brachiis orientalibus jugi Rushani, $200 \mathrm{~m}$ west a trajectu Koi-Tezek, in declivi arduo SE expositionis, inter fragmina lapidum in detritis mobilibus, $37^{\circ} 29^{\prime} \mathrm{N}, 72^{\circ} 44^{\prime} \mathrm{E}, 4022 \mathrm{~m}$ a. s. 1. 02 VIII 2011. Kljuykov, Zakharova \& Ukrainskaja 20" (holotype MW! [MW0594098], isotypes K [K001096687], MW [MW0594099]).

Distribution in MA \& K: Tajikistan (Kŭhistani Badakhshan). Endemic.

Distribution area: Central Asia.

Semenovia furcata Korovin, 1973, Izv. Akad. Nauk Tadzh. SSR, Otd. Estest. Nauk 2(51): 11.

Type: TAJIKISTAN. "Pamir, vall. fl. Bilandkiik, fl. Sel-Dara. 18 VIII 1958. Sidorov 8152" (holotype TAD!).

Distribution in MA \& K: Tajikistan (Kŭhistani Badakhshan, Dushanbe prov.). Endemic.

Distribution area: Central Asia.

Semenovia heterodonta (Korovin) Manden. 1959, Trudy Tbilis. Bot. Inst. 20: 22.

$\equiv$ Platytaenia heterodonta Korovin, 1947, Not. Syst. Herb. Inst. Bot. Acad. Sci. Uzbekistan 8: 5.

三Zosima heterodonta (Korovin) M. Hiroe, 1979, Umbelliferae World: 1762.

Type: UZBEKISTAN. "Pamir-Alaj, montes Turkestanici, vallis fl. Sansar, Guralasch-saj, 08 VIII 1937, Korotkova 872" (holotype TASH - not found).

- Zosima tragioides auct. non Boiss.: O. et B. Fedtsch., 1909, Consp. F1. Turkest. (O. A. Fedtschenko et B. A. Fedtschenko) 3: 113.

Distribution in MA \& K: Tajikistan (Sughd prov., Dushanbe prov.), Uzbekistan (Dzhizak prov., Surxondarya prov.). Endemic.

Distribution area: South-Western Asia.

Note: The type of this species name has not been found in joint TASH herbarium so far. It was written in protologue, that it was deposited at "Herb. So. Uzbek. in Taschkent".

Semenovia imbricata Ukrainskaja et Kljuykov, 2013, Nordic J. Bot. 31(6): 652, Fig. 3, 5-7.

Type: TAJIKISTAN. "Tadshikistania, Badachschan region autonoma, Schugnan districtus, in brachiis septentrionalibus jugi Schugnani, ripa sinistra fluvii Gunt, ostium fluvii Duzachdara, haud procul ab influxi fluminis Toguzbulak, in rupibus NE expositionis, 370 $40^{\prime} \mathrm{N}, 72^{\circ} 30^{\prime} \mathrm{E}$. 01 VIII 2011. Kljuykov, Zakharova \& Ukrainskaja 13" (holotype MW! [MW0594100], isotypes K [K001096685], MW [MW0594101]).

Distribution in MA \& K: Tajikistan (Kŭhistani Badakhshan).), Afghanistan (NE: Badakhshan). 


\section{Distribution in other Asian countries: Afghanistan.}

Distribution area: South-Western Asia.

Semenovia pamirica (Lipsky) Manden. 1959, Trudy Tbilis. Bot. Inst. 20: 22.

$\equiv$ Zosima pamirica Lipsky, 1903, Vidensk. Medd. Dansk Natur. Foren Kjobenhavn 1903: 143.

$\equiv$ Platytaenia pamirica (Lipsky) Nevski et Vved. 1947, Trudy Bot. Inst. Akad. Nauk SSSR, ser. 1 F1. Sist. Vyssh. Rast. 4: 271.

$\equiv$ Neoplatytaenia pamirica (Lipsky) Geld. 1990, in V. V. Niktin et A. M. Geldikhanov, Opred. Rast. Turkmenistana: 455; 1988, in Bot. Zhurn. (Leningrad) 75, 5: 711, IX sine basion. compl.

Type: TAJIKISTAN. "Pamir, in angustiis Chargush, 4300 m, 04 IX 1898, Paulsen 1254", (syntypes C! [C10008556, C10008557], LE [LE00052210]); "Chugnan: Masar in Chakh-dara, in declivibus lapidosis, 25. VII 1897 Korshinsky 3181" (lectotype LE! [LE00052211], designated by V. M. Vinogradova (2002: 138); isolectotypes LE [LE00052212, LE00052213]).

Distribution in MA \& K: Tajikistan (Kŭhistani Badakhshan).

Distribution in other Asian countries: Afghanistan.

Distribution area: Central and South-Western Asia.

Semenovia pimpinelloides (Nevski) Manden., 1959, Trudy Tbilis. Bot. Inst. 20: 22.

$\equiv$ Platytaenia pimpinelloides Nevski, 1937, Trudy Bot. Inst. Akad. Nauk SSSR, ser. 1 (Fl. Sist. Vyssh. Rast.) 4: 271.

$\equiv$ Zosima pimpinelloides (Nevski) M. Hiroe, 1979, Umbelliferae World: 1761.

$\equiv$ Neoplatytaenia pimpinelloides (Nevski) Geld., 1988, in V. V.Nikitin Niktin et A. M. Geldikhanov, Opred. Rast. Turkmenistana: 455; 1990, Bot. Zhurn. (Leningrad) 75(5): 711 .

Type: UZBEKISTAN. "In pratis stepposis lapidosis cum Eremuro kauffmanii sub cacuminibus montium Kuhitang supra pagum Chodsha-i-fil. 17 VII 1931. Nevski 883" (holotype LE! [LE00052231], isotype LE [LE00052230]).

Distribution in MA \& K: Tajikistan (Sughd prov.), Uzbekistan (Dzhizak prov., Qashqadarya prov., Surxondarya prov.), Turkmenistan (Lebap prov.).

Distribution in other Asian countries: China.

Distribution area: South-Western Asia.
Semenovia pulvinata Pimenov et Kljuykov, 2013, Nordic J. Bot. 31(6): 649, Fig. 1.

Type: TAJIKISTAN. "Tadshikistania, Pamir orientalis, in systemate fluminis Muksu, prata in cursu inferiore fluminis Kaindy. 20 VIII 1936. Stanjukovich 372" (holotype TASH! [TASH002583]).

Distribution in MA \& K: Tajikistan (Kŭhistani Badakhshan). Endemic.

Distribution area: Central Asia.

Semenovia rubtzovii (Schischk.) Manden., 1959, Trudy Tbilis. Bot. Inst. 20: 23.

$\equiv$ Platytaenia rubtzovii Schischk., 1951, in Schischkin (ed.), Fl. URSS 17: 357, 273, tab. 23, fig. 5.

三 Zosima rubtzovii (Schischk.) Kamelin, 1977, Novit. Syst. P1. Vasc. 14: 192. 1977.[

Type: KAZAKHSTAN. "Kasachstania, Montes Dzhungarski Alatau. Ditio Dzharkent, in faucibus Taldy-bulak, 18 VII 1937, Rubtzov \& Linczevski” (holotype LE [LE00052232]; isotype AA!).

Distribution in MA \& K: Kazakhstan (SE: Almaty prov.).

Distribution in other Asian countries: China.

Distribution area: Central \& South-Western Asia.

Note: The species occupies an isolated position in the genus Semenovia, and, in many ways, is closer to the genus Zosima (Kamelin, 1977)

Semenovia tragioides (Boiss.) Manden., 1959, Trudy Tbilis. Bot. Inst. 20: 21.

$\equiv$ Zosima tragioides Boiss., 1844, Ann. Sci. Nat. sér. 3, 1: 341.

$\equiv$ Heracleum tragioides (Boiss.) Benth. et Hook. f. ex C. B. Clarke, 1879, in Fl. Brit. Ind. (J. D. Hooker) 2(6): 712.

$\equiv$ Platytaenia tragioides (Boiss.) Nevski et Vved. 1937, Trudy Bot. Inst. Akad. Nauk SSSR, ser. 1 Fl. Sist. Vyssh. Rast. 4: 271, in obs.

Type: IRAN. "In Persia, Aucher-Eloy 3702" (lectotype G-BOIS!; designated by Pimenov \& Jacquemoud; (2020: 70), FI! [FI015039], G! [G00370265, G00370266], K! [K000685767], MPU [MPU021016], P [P00752733, P00757877]).

- S. dichotoma auct. non Manden.: Pimenov, 1983, in Vvedensky (ed.), Opred. Rast. Sred. Azii 7: 319 .

Distribution in MA \& K: Turkmenistan (Balkan prov., Akhal prov.).

Distribution in other Asian countries: Iran.

Distribution area: South-Western Asia. 
Note: Lipsky (1904:154) showed Zosima tragioides Boiss. for vicinities of Yaschilkul lake in Pamirs (collections of O.W. Paulsen and B. A. Fedtschenko) and noted the presence of two forms, differing in the dissection of the leaves, in the species. At the same time, Z. tragioides (Semenovia tragioides) is not given from the territory of Pamirs and grows in Middle Asia only in highlands of Kopetdagh (Turkmenistan), while the only species of Semenovia at the lake Yaschilkul is S. pamirica.

Semenovia transiliensis Regel et Herder, 1866, Bull. Soc. Imp. Naturalistes Moscou 39(2): 79.

$\equiv$ Heracleum transiliense (Regel et Herder) O. et B. Fedtsch., 1909, Consp. Fl. Turkest. (O. A. Fedtschenko et B. A. Fedtschenko \& B. A. Fedtschenko) 3: 112 .

Type: KAZAKHSTAN. "Ober Tschilik Thal Alatau transiliensis, 23 VII 1857, Semenov" (lectotype LE! [LE00052241], designated by V. M. Vinogradova (2002: 134); isolectotypes LE [LE00052242LE00052244], US [US00517444]); "Alatau transiliensis, Kurmetu Pass, 1857, Semenov" (syntypes LE [LE0005172, LE0005173, LE00052240]).

= Heracleum pimpinellifolium Rupr., 1869, Mém. Acad. Imp. Sci. Saint-Petersbourg, (ser. 7), 14(4) (Sert. Tianschan.): 49, nom. illeg. (Art. 52.1), non Spreng. (1813).

Type: KYRGYZSTAN. "Schamsi-Schlucht, 09 VII 1867, Osten-Saken" (holotype LE! [LE00052209]).

= Heracleum angustilobatum Nikitina, 1959, in Vvedensky (ed.), Fl. Kirgiz. SSR 8: 107, nom. inval. (Art. 39.1); id. ibid., 1960, 9: 207, descr.

Type: KYRGYZSTAN. "Bassin fl. Chon-Kemin, loco Karagailun-Bulak, declivum septentrionaleoccidentalis, prata. 22 VI 1954. Isakov" (holotype FRU!).

Distribution in MA \& K: Kazakhstan (SE: Almaty prov.), Kyrgyzstan (Ysyk-köl prov., Naryn prov., Chüh prov.).

Distribution in other Asian countries: China.

Distribution area: Central and South-Western Asia.

Semenovia vachanica Ukrainskaja et Kljuykov, 2013, Nordic J. Bot. 31(6): 654, Fig. 4-7.

Type: TAJIKISTAN. "Tadshikistania, Badachschan region autonoma, Ischkaschim districtus, declive occidentalis jugi Ischkaschimi, ad ripam fluminis Boguschdara, $6 \mathrm{~km}$ supra vicum Bolo, in declivi expositionis septentrionalis, in caespitio et in glareosis, $37^{\circ} 03.5^{\prime} \mathrm{N}, 71^{\circ} 34^{\prime} \mathrm{E}, 3000$ $\mathrm{m}$ a. s. 1. 05 VIII 2011. Kljuykov, Zakharova \& Ukrainskaja 28" (holotype MW! [MW0594102], isotypes K [K001096686], MW [MW0594103]).

Distribution in MA \& K: Tajikistan (Kŭhistani Badakhshan).

Distribution in other Asian countries: Afghanistan.

Distribution area: Central and South-Western Asia.

Semenovia vaginata Pimenov, 2008, Novit. Syst. Pl. Vasc. 40: 192.

Type: KYRGYZSTAN. "Kyrgyzstan, provincia Osch, declivum septentrionale jugi Turkestanici, in vicinitate pagi Andigen, locus Sary-jaz, 3949'N, $70^{\circ} 30^{\prime}$ E. 15 VII 1969. Pimenov, Borjaev, Trusov \& Saprunova 1254" (holotype MW! [MW0594104], isotype LE! [LE00052246, LE00052247]).

Distribution in MA \& K: Kyrgyzstan (Batken prov.). Endemic

Distribution area: South-Western Asia.

Semenovia zaprjagaevii Korovin, 1973, Izv. Akad. Nauk Tadzhiksk. SSR, Otd. Estest. Nauk 2(51): 12 .

Type: KYRGYZSTAN. "Jugum Turkestanicum, pars septentrionalis, in angustis Orgli, 2100 m s. m., 07 VII 1954, Zaprjagaeva" (holotype TAD!).

Distribution in MA \& K: Kyrgyzstan (Batken prov.), Tajikistan (Sughd prov.). Endemic.

Distribution area: South-Western Asia.

Seseli abolinii (Korovin) Schischk., 1950, in Schischkin (ed.), Fl. URSS 16: 505, tab. 34, fig. 5.

$\equiv$ Phlojodicarpus abolinii Korovin, 1924, Bot. Mater. Gerb. Glavn. Bot. Sada R.S.F.S.R 5(6): 74.

$\equiv$ Libanotis abolinii (Korovin) Korovin, 1963, inN.V. Pavlov (ed.), Fl. Kazakhst. 6: 351.

Type: KAZAKHSTAN. "Prov. Heptapotamica, jugum Dzhungarsky Alatau, montes Karu-Duvan, valle fl. Borochudsir, decl. saxosum, 28 VI 1917, Abolin, Borman \& Schamberg 4165" (lectotype TASH! [TASH002368], designated by Pimenov 1978: 189); "Prov. Heptapotamica, distr. Dzharkent, Dzhungarski Alatau, Mts. Kok-dzeta, riv. W Usek, Taldy-su. 02 VIII 1917. Abolin, Borman \& Schtamberg 4801" (syntypes LE, TASH [TASH002367]; "Prov. Heptapotamica, distr. Verny, Mt. Turaigyr, trajectum Terekty. 11 VII 1917. Abolin 3488, 3552" (syntypes TASH [TASH002365, TASH002366]; "Prov. Heptapotamica, distr. Dzharkent, Dzhungar- 
ski Alatau, Mt. Suat-tau, Kara-sai. 03 VIII 1917, $A b$ olin, Borman \& Schtamberg 4925" (syntype TASH [TASH002364]).

$=$ Seseli songoricum Schischk., 1950, in Schischkin (ed.), Fl. URSS 16: 602, 505.

$=$ Libanotis soongorica (Schischk.) Korovin, 1963, in Pavlov (ed.), Fl. Kazakhst. 6: 350.

Type: KAZAKHSTAN. "Dzhungarski Alatau, Chaptzagai meridionalis, ad ripam affluentis contra Karaschar, alt. ca. 2400 m, 30 VIII 1879, A. Regel" (lectotype LE! [LE01066012], designated by Schischkin $(1950 ; 602)$; isolectotypes LE! [LE01066010, LE01066011]) VIII 1879, A. Regel' (holotype LE!)

= Libanotis michaylovae Korovin, 1962, Trudy Inst. Bot. Akad. Nauk Kazakh. SSR 13: 254.

Type: KAZAKHSTAN. "Montes DshungarskyAlatau, pars meridionalis, in angustic Suak-su, ad locos lapidosos, 22 VIII 1938, Michaylova" (holotype AA!).

= Libanotis turajgyrica Bajtenov, Vestnik 1968, Vestn. Akad. Nauk Kazakh. SSR 7 (279): 67.

Type: KAZAKHSTAN. "Transiliensi Alatau, montes Turajgyr, mons Serektas ad declivitate lapidosas, 29 VIII 1967, Bajtenov" (holotype AA!; isotype LE! [LE01066055]).

= Seseli langshanense Zhao Yizhi et Ma Yuchuan, 1991, Acta Sci. Nat. Univ. Intramongol. 22(3): 407.

Type: CHINA. "Nei Mongol: Bayannaoermen, Langshan, Hulougaier, in vallibus vel clivis petrosis, alt. 1800-2000 m, 07 VII 1988, Zhao Yizhi et al. 2197" (holotype HIMC).

Distribution in MA \& K: Kazakhstan (SE: Almaty prov.).

Distribution in other Asian countries: China, Mongolia

Distribution area: Central and South-Western Asia.

Seseli aemulans Popov, 1940, Bot. Mat. Gerb. Bot. Inst. Komarova Akad. Nauk SSSR 8(4): 73.

Type: KAZAKHSTAN. "Alatau transiliensis, ad fl. Tscharyn, in deserto saxoso gypsaceo (hamada) prope pag. Tscharyn, inter frutices Caraganae aurantiacae, cum Sisymbrio subspinescenti, Anabasi etc., 09 VIII 1937, Popov" (lectotype LE! [LE01065731]; designated by Goloskokov (1963: 52); isolectotype AA!).

Distribution in MA \& K: Kazakhstan (S: Zhambyl prov.; SE: Almaty prov.).

Distribution in other Asian countries: China.

Distribution area: Central and South-Western Asia.
Seseli afghanicum (Podlech) Pimenov, 1977, Byull. Moskovsk. Obshch. Isp. Prir. Otd. Biol. 82(3): 140.

三Libanotis afghanica Podlech, 1970, Mitt. Bot. Staatssamml. München 8: 171.

Type: AFGHANISTAN. "Nordost-Afghanistan, Prov. Badakhshan: Oberes Anjuman-Tal, Umgebung des Ortes Anjuman, 3100 m, 14 VIII1965, Podlech 12358" (holotype M! [M0172928]; isotypes E! [E00002743], LE!, MSB [MSB003203]).

= Seseli subaphyllum Korovin, 1975, Izv. Akad. Nauk Tadzh. SSR, Otd. Estest. Nauk 3(60): 6.

Type: TAJIKISTAN. "Systema fl. Pjandzh, vallis fl. Bidzhun-Dara, prope pagum Bidzhun-Dara. 21 VII 1936. Nikiforova \& Kuznetzov 310" (holotype TASH [TASH002316]).

Distribution in MA \& K: Tajikistan (Kŭhistani Badakhshan).

Distribution in other Asian countries: Afghanistan.

Distribution area: South-Western Asia.

Seseli alaicum Pimenov, 1990, Byull. Moskovsk. Obshch. Isp. Prir. Otd. Biol. 95(1): 102, fig. 1.

Type: KYRGYZSTAN. "Kyrgyzstan, jugum Alaicum, declivum septentrionale. Mts. Arpalyk, angustis Dundurek-dange, in fissuris rupium, $11 \mathrm{VII}$ 1987, Pimenov 237" (holotype MW! [MW0593854]; isotypes LE! [LE01065733, LE01065734]).

Distribution in MA \& K: Kyrgyzstan (Batken prov.). Endemic.

Distribution area: South-Western Asia.

Seseli asperulum (Trautv.) Schischk., 1950, in Schischkin (ed.), Fl. URSS 16: 520, tab. 34, fig. 7.

$\equiv$ Seseli coronatum Ledeb. var. asperulum Trautv. 1871, Trudy Imp. S.-Peterburgsk. Bot. Sada 1: 32).

Type: KAZAKHSTAN. "Songoria, 1869, Hb. Trautvetter" (lectotype LE! [LE01065944], designated as "typus" by Pimenov (1978:195); isolectotypes LE! [LE01065945-LE01065947].

Distribution in MA \& K: Kazakhstan (NE: E Kazakhstan prov.; SE: Almaty prov.).

Distribution in other Asian countries: China.

Distribution area: Central Asia.

Seseli betpakdalense Bajtenov, 1974, Bot. Mater. Gerb. Inst. Bot. Akad. Nauk Kazakh. SSR 8: 36.

Type: KAZAKHSTAN. "Betpak-Dala, fl. Saryssu, in argillis rubris denudates. 24 IX 1954. $B a$ jtenov 927" (holotype AA).

Distribution in MA \& K: Kazakhstan (Central: Karagandy prov.). Endemic. 


\section{Distribution area: Central Asia.}

Seseli buchtormense (Spreng.) W. D. J. Koch, 1824, Nova Acta Phys.-Med. Acad. Caes. Leop.Carol. Nat. Cur. 12(1): 111.

$\equiv$ Bubon buchtormensis Spreng., 1815, P1. Min. Cogn. Pug. 2: 55.

$\equiv$ Libanotis buchtormensis (Spreng.) DC., 1829, Col. Mém. 5 (Mém. Fam. Ombell.): 48, tab. 3, fig. S.

$\equiv$ Hippomarathrum buchtormense (Spreng.) Link, 1829, Handbuch (Link) 1: 349.

三 Balinotella buchtormensis (Spreng.) Soják, 1982, Zprávy Krajsk. Vlastiv. Muz. Olomouci 215: 4.

Type: RUSSIA/KAZAKHSTAN. "In Sibiria [Ex Hort. Bot. Berol.]" (holotype B?).

$=$ Athamanta rigida Hornem. 1815, Hort. Bot. Hafn. 2: 960.

Type: not indicated.

= Seseli giraldii Diels, 1900, Bot. Jahrb. Syst. 29 (3-4) (Fl. Centr. China): 497.

Type: CHINA. "N, Kuan to shan [Kuan-to-san, Shen-si septent. (Cina)]. XI 1896. Giraldi 1335" (holotype FI!).

= Libanotis cycloloba Gilli, 1959, Repert. Spec. Nov. Regni Veg. 61(3): 196.

= Seseli cyclolobum (Gilli) Pimenov et Sdobnina, 1975, Bot. Zhurn. (Leningrad) 60(8): 1120.

Type: AFGHANISTAN. "Ost-Afghanistan: Gebirge oberhalb des zwischen Gulbahar und Surobi gelegenen Nedschrab-(Nidjrau-Tales [Kabul, Nijrao, Gebirge oberhalb des Nedschrabtales], enge Stelle in einer Schlucht, durch die ein Bächlein wasserfallartig herunterstürzt, in einer schwer erreichbaren Felsspalte, 3100 m, 13 IX 1951 Gilli 1992" (holotype W!).

- Seseli sibiricum auct. non Garcke: Benth. ex C. B. Clarke, 1879, in F1. Brit. India (J. D. Hooker) 2: 693 .

- Seseli libanotis auct. non W. D. J. Koch: Nasir, 1972, in Nasir et Ali (ed.), Fl. W Pakistan 20: 76.

Distribution in MA \& K: Kazakhstan ( $\mathrm{N}$ : Pavlodar prov.; NE: E Kazakhstan prov.; Central: Karagandy prov.; SE: Almaty prov.), Kyrgyzstan (Ysyk-köl prov.).

Distribution in other Asian countries: Russia, China, India, Pakistan, Mongolia, Afghanistan.

Distribution area: Northern, Central, Eastern and South-Western Asia.

Seseli calycinum (Korovin) Pimenov et Sdobnina, 1975, Bot. Zhurn. (Leningrad) 60(8): 1118.
E Libanotis calycina Korovin, 1947, Not. Syst. Herb. Inst. Bot. Zool. Acad. Sci. Uzbekistan 8: 20.

E Balinotella calycina (Korovin) Soják, 1982, Zprávy Krajsk. Vlastiv. Muz. Olomouci 215: 4.

Type: UZBEKISTAN. "Tian-Schan occidentalis, mons Tschimgan, Korovin 136" (holotype TASH! [TASH002318]).

Distribution in MA \& K: Kyrgyzstan (JalalAbad), Uzbekistan (Namangan prov., Toshkent prov.), Tajikistan (Sughd prov.). Endemic.

Distribution area: South-Western Asia.

Seseli condensatum (L.) Rchb. f. 1867, in Icon. F1. Germ. Helv. (Reichenbach) 21: 37.

三Athamanta condensata L., 1753, Sp. Pl.: 1195.

$\equiv$ Libanotis condensata (L.) Crantz, 1767, Cl. Umb. Emend.: 106.

三Ligusticum condensatum (L.) Spreng., 1813, Neue Schr. Naturf. Ges. Halle 2(1) (P1. Umb. Prodr.): 40.

E Libanotis vulgaris DC. var. condensata (L.)

DC., 1830, Prodr. (A. P. de Candolle) 4: 150.

E Peucedanum condensatum (L.) Koso-Pol., 1922, Sched. Herb. F1. Ross. 8: 115.

$\equiv$ Pachypleurum condensatum (L.) Korovin, 1963, in Pavlov (ed.), Fl. Kazakhst. 6: 310.

Type: RUSSIA. "[Described on plants, grown at Uppsala from seeds, collected by P. G. Demidov in "Siberia", probably near Solikamsk], 345.5" (lectotype LINN [LINN-HL 345-5], designated by Jarvis et Knees in Taxon 37(2): 474 (1988)).

= Ligusticum vaginatum Spreng., 1815, P1. Min. Cogn. Pug. 2: 57.

= Libanotis fastigiata Rupr., 1845, Beitr. Pflanzenk. Russ. Reiches 2: 36, in textu.

$=$ Libanotis arctica Rupr., 1845, Beitr. Pflanzenk. Russ. Reiches 2: 36.

= Seseli arcticum (Rupr.) Calest. 1905, Webbia 1: 203. 1905.

= Libanotis condensata (L.) Crantz subsp. arctica (Rupr.) V. G. Sergienko, 1980, Novit. Syst. P1. Vasc. 17: 245.

Type: RUSSIA. "[N. Ural] Epromont, Mikulkin" (holotype LE?).

= Carum takenakai Kitag. 1951, J. Jap. Bot. 26(6): 166.

Type: CHINA. "China: Prov. Hepei: in monte Hsiao-wu-tai-shan, VIII 1938, Takenaka 88”, (holotype TI!).

Distribution in MA \& K: Kazakhstan (NE: E Kazakhstan prov.; SE: Almaty prov.).

Distribution in other Asian countries: Russia, China, Mongolia. 
Distribution area: Asia (Arctic, Norhtrn, Central and Eastern), Europe (E).

Seseli coronatum Ledeb., 1829, Fl. Altaic. (Ledebour) 1: 336.

Type: KAZAKHSTAN. "[Altai] In deserto soongoro-kirghizico, 1826, Meyer 264" (lectotype LE! [LE01065948]; designated as "typus" by Pimenov (1978: 195); isolectotypes C [C10008562], LE! [LE01065949], P! [P02496371]).

- Seseli tschuiliense auct. non Korovin: Yang Chunyu, 1985, Clav. Pl. Xinjiang. 3: 403.

- Hyalolaena tschuiliensis auct. non Pimenov et Kljuykov: V. M. Vinogr. 1994, in Rast. Tsentral'noi Azii 10: 57.

Distribution in MA \& K: Kazakhstan (N: Akmola prov.; NE: E Kazakhstan prov.).

Distribution in other Asian countries: China.

Distribution area: Central Asia.

Seseli desertorum Pimenov, 1977, Byull. Moskovsk. Obshch. Isp. Prir. Otd. Biol. 82(3): 138.

Type: UZBEKISTAN. "Kysyl-Kum, inter pag. Kok-petas et pag. Djus-Kuduk, in arenosis, 06 VII 1932, Russanov 346" (lectotype LE! [LE01065954]; designated as "typus" by Pimenov (1977: 139); Kysil-Kum, montes Kok-patas, in arenosos. 08 VI 1932. Muravlinsky (paratype LE).

Distribution in MA \& K: Uzbekistan (Navoi prov.). Endemic.

Distribution area: Central and South-Western Asia.

Seseli eriocarpum (Schrenk) B. Fedtsch., 1915, Rastit. Turkest.: 617.

$\equiv$ Libanotis eriocarpa Schrenk, 1843, Bull. Phys.-Math. Acad. Petersb. 2: 195.

Type: KAZAKHSTAN. "Songarei, grasreiche Steppen am Kara-Kingir, 10 VI 1842, Schrenk 621" (syntypes LE! [LE01066036, LE01066037]); "In collibus aridis mont. Ulutau, Schrenk 622" (lectotype et isolectotypes LE! [LE01066038LE01066042]; designated as "typus" by Pimenov (1978: 189); isolectotype MANCH!).

Distribution in MA \& $\mathbf{K}$ : Kazakhstan $(\mathrm{N}$ : Kostonai prov., Akmola prov., Pavlodar prov.; Central: Karagandy prov.).

Distribution in other Asian countries: China, Mongolia.

Distribution area: Central Asia.

Seseli eriocephalum (Pall. ex Spreng.) Schischk., 1950, in Schischkin (ed.), Fl. URSS 16: 518, tab. 34, fig. 3. $\equiv$ Bubon eriocephalus Pall. ex Spreng., 1824, Syst. Veg. (ed. 16) 1: 900.

三Seseli lessingianum Turcz. ex Kar. et Kir. var. eriocephalum (Pall. ex Spreng.) O. et B. Fedtsch., 1909, Consp. Fl. Turkest. (O. A. Fedtschenko et B. A. Fedtschenko) 3: 92.

Type: KAZAKHSTAN. "Sibir, ad lacum Inder, Pallas" (holotype LINN).

= Seseli lessingianum Turcz. ex Kar. et Kir., 1841, Bull. Soc. Imp. Naturalistes Moscou 14: 429.

$=$ Lomatopodium lessingianum (Turcz. ex Kar. et Kir.) Fisch. et C. A. Mey. 1845, Bull. Phys.-Math. Acad. Petersb. 3: 305.

Type: KAZAKHSTAN. "In deserto Kirghisico orientaliore [Deserto Kirgisorum], Lessing" (lectotype LE! [LE01065978], designated by V. M. Vinogradova (2002: 135); isolectotypes LE! [LE01065979-LE01065981], MW! [MW0593867]).

= Lomatopodium platyphyllum Schrenk, 1845, Bull. Phys.-Math. Acad. Petersb. 3: 306.

= Seseli platyphyllum (Schrenk) O. et B. Fedtsch., 1909, Consp. Fl. Turkest. (O. A. Fedtschenko \& B. A. Fedtschenko) 3: 94. 1909.

Type: KAZAKHSTAN. "Songaria, Balchasch. [In litore fluvii Chatynssu], 23-25 VII 1843, Schrenk" 347 (lectotype LE! [LE01066074]; designated here by Vinogradova (2001: 53); isolectotypes BP!, G! [G00367368], JE! [JE00019224], K!, LE [LE01066075, LE01066080], MANCH!, MW! [MW0593852]); "Songaria, 23 VII 1847. Schrenk" (syntypes LE! [LE01066077-LE01066083]).

= Lomatopodium karelinianum Turcz. ex Stschegl., Bull. Soc. Imp. Naturalistes Moscou 27(1): 167. 1854.

Type: KAZAKHSTAN. "In salsis deserti Soongaro-Kirghisici inter Kurtschum et lacum Noor-Saissan, 1840, Karelin \& Kirilov" (lectotype LE! [LE01066063, LE01066064],, designated by I. A. Gubanov et al. (1998: 64); isolectotypes LE! [LE01066065], MW! [MW0693866, MW0593867]).

Distribution in MA \& K: Kazakhstan (W: W Kazakhstan prov., Atyrau prov., Aktobe prov., Mangystau prov.; N: Akmola prov.; NE: E Kazakhstan prov.; Central: Karagandy prov.; S: S Kazakhstan prov., Zhambyl prov.; SE: Almaty prov.), Uzbekistan (Kara-kalpak).

Distribution in other Asian countries: Russia, China.

Distribution area: Central Asia.

Seseli eryngioides (Korovin) Pimenov et V. N. Tikhom., 1981, in S. K. Cherepanov, Sosud. Rast. 
SSSR: 30. $\equiv$ Sphenocarpus eryngioides Korovin, 1947, Not. Syst. Herb. Inst. Bot. Zool. Acad. Sci. Uzbekistan 8: 23.

Type: KYRGYZSTAN. "Fergana orientali s, vallis fluv. Itokar, 17 IX 1927, Sovetkina 1260" (holotype TASH! [TASH002331]; isotype LE! [LE01066062 - only fruits]).

Distribution in MA \& K: Kyrgyzstan (JalalAbad prov.). Endemic.

Distribution area: South-Western Asia.

Seseli fasciculatum (Korovin) Korovin ex Schischk., 1950, in Schischkin (ed.), Fl. URSS 16: 507, p. p.

$\equiv$ Libanotis fasciculata Korovin, 1926, Byull. Sredne-Asiatsk. Gosud. Univ. 14, Suppl. (Sched. Herb. Fl. As. Med. 10): 11, N 240.

Type: UZBEKISTAN. "Syr-Darja, distr. Taschkent, ad declivia saxosa in regione subalpina montis Tschimgan Majoris, 30 VII 1925, Korovin \& Mokeeva 240" [Sched. Herb. Fl. Asia Med 10: N 240] (lectotype TASH! [TASH002319[TASH002319; designated as "typus" by Pimenov (1978: 192); isolectotypes B! [B 10 0367282], BP!, C! [C10008564], G! [G00367366], K! [K000697450], LE! [LE01066050-LE01066052], MO, MW! [MW0593851], NY [NY00406068], P! [P02496391], S! [S-G-3660], Z [Z-000028693]).

Distribution in MA \& K: Kazakhstan (S: S Kazakhstan prov., Zhambyl prov.), Kyrgyzstan (Jalal-Abad prov., Talas prov.), Uzbekistan (Tashkent prov.), Tajikistan (Sughd prov.). Endemic.

Distribution area: South-Western Asia.

Seseli galioides Lazkov, 2008, Novit. Syst. Pl. Vasc. 40: 226.

Type: KYRGYZSTAN. "Jugi Ferganici declivum generale boreale, riv. Karasu, in rupibus, alt. $1300 \mathrm{~m} \mathrm{s.} \mathrm{m.,} 41^{\circ} 39^{\prime} 23^{\prime \prime}$ lat. bor., $72^{\circ} 49^{\prime} 23^{\prime \prime}$ long. or. 04 VIII 2006. Lazkov" (holotype LE! [LE01065955]; isotypes FRU, MW [MW0593859]).

Distribution in MA \& K: Kyrgyzstan (JalalAbad prov.). Endemic.

Distribution area: South-Western Asia.

Seseli giganteum Lipsky, 1904, Trudy Imp. S.Peterburgsk. Bot. Sada 23: 140.

Type: KYRGYZSTAN. "Tian-Schan occidentalis, vallis fl. Chodzha-ata, in vallis riv. Karanchutun, 23 VIII 1902, B. A. Fedtschenko" (lectotype LE! [LE01065956], designated as type by Pimenov (1974: 242); isolectotypes LE! [LE01065957, LE01065958].
Distribution in MA \& K: Kyrgyzstan (JalalAbad prov.). Endemic.

Distribution area: South-Western Asia.

Seseli glabratum Willd. ex Spreng., 1820, in Syst. Veg. ed. 15 bis (Roemer et Schultes) 6: 406.

Type: RUSSIA. "In Sibiria, Pallas W5945" (holotype B-Willd!).

= Seseli tenuifolium Ledeb., 1829, Fl. Altaic. (Ledebour) 1: 333; 1829, Icon. Pl. Alt. 1: 23, tab. 97.

Type: KAZAKHSTAN. "In apricis siccis sterilibus prope fortalitium Ustkamenogorsk [locis apricis pr. Ustkamenogorsk et simelibus locis in deserto occidental. trans. fl. Irtysch], Meyer 260" (lectotype LE! [LE01066016], designated by V. M. Vinogradova (2002: 136); isolectotypes B, C! [C10008566?], HAL [HAL0098518], LE! [LE01066017-LE01066019]); "In deserto Kirghisorum inter Ustkamenogorsk et Ablaikit, Meyer 379" (syntypes LE, P [P02496622, $\mathrm{P} 02496623])$.

Distribution in MA \& K: Kazakhstan (W: W Kazakhstan prov., Atyrau prov., Aktobe prov., Mangystau prov.; N: Kostonai prov., Akmola prov., Pavlodar prov.; NE: E Kazakhstan prov.; Central: Karagandy prov.), Uzbekistan (Kara-kalpak).

Distribution in other Asian countries: Russia, China, Mongolia.

Distribution area: Central Asia.

Seseli incanum (Steph. ex Willd.) B. Fedtsch., 1915, Rastit. Turkest.: 617.

$\equiv$ Athamanta incana Steph. ex Willd. 1798, Sp. Pl. ed. 4 (Willdenow) 1 (2): 1402.

$\equiv$ Libanotis incana (Steph. ex Willd.) O. et B. Fedtsch., 1909, Consp. Fl. Turkest. (O. A. Fedtschenko \& B. A. Fedtschenko) 3: 94.

Type: KAZAKHSTAN. "Habitat in Sibiria [Altay, near Ust-Kamenogorsk], Stephan" (holotype B-Willd!).

= Libanotis patriniana DC., 1830, Prodr. (A. P. de Candolle) 4: 150.

Type: KAZAKHSTAN. "In collo schistoso arido ad ripam flum. Irtisch, in Sibiria altaica ad arcem Oust-Kamenogorsk, Patrin".

= Seseli graveolens Ledeb., 1829, Fl. Altaic. (Ledebour) 1: 340; id., 1830, Icon. Pl. Altaic. 3: 6, tab. 104, nom. illeg. (Art. 53.1), non Scop. (1771).

$\equiv$ Libanotis graveolens $\mathrm{G}$. Don, 1834, Gen. Hist. 3: 312 .

Type: KAZAKHSTAN. "In rupestribus rarius prope fortalitium Ustkamenogorsk, nec non frequens trans.fl. Irtysch, versus ruinas Ablakit, 1826, Meyer 
179" (lectotype LE! [LE01065959], designated by V. M. Vinogradova (2002: 135); isolectotypes B [B 10 0367278?], HAL [HAL0098527], LE [LE01065960, LE01065961]).

Distribution in MA \& K: Kazakhstan (NE: E Kazakhstan prov.; Central: Karagandy prov.).

Distribution in other Asian countries: China.

Distribution area: Central Asia.

Seseli karateginum Lipsky, 1904 Trudy Imp. S.-Peterburgsk. Bot. Sada 23: 141.

Type: TAJIKISTAN. "Karategin, ad fl, Surchob prope Damburashi, 30 VII 1897, Lipsky 1497" (lectotype LE! [LE01065967; designated as "typus" by Pimenov (1978: 190); isolectotypes LE! [LE01065968, LE01065969]).

- Seseli lehmannianum auct. non Boiss.: Kaschtsch. 1959, in Vvedensky (ed.), Fl. Kirgh. SSR 8: 69, p. p.

Distribution in MA \& K: Kyrgyzstan (Batken prov., Osh prov.), Tajikistan (Dushanbe prov.). Endemic.

Distribution area: South-Western Asia.

Seseli kaschgaricum Pimenov et Kljuykov, 1992, Bot. Zhurn. (St. Perersburg) 77(2): 94, fig. 2.

Type: KYRGYZSTAN. "Asia Media, Kyrgyzstan, Tian-Schan Orientalis, in systemate fluminis Sarydzhaz, rivulus Ucz-czat ad meridiem a Taschkoroo, 14 VIII 1988, Pimenov \& Kljuykov 601" (holotype MW! [MW0593865]; isotypes LE! [LE01065971], MW [MW0593863, MW0593864]).

Distribution in MA \& K: Kyrgyzstan (Ysykköl prov.). Endemic.

Distribution area: Central Asia.

Seseli korovinii Schischk., 1950, Bot. Mater. Gerb. Bot. Inst. Komarova Akad. Nauk SSSR 13: 162.

$\equiv$ Libanotis korovinii (Schischk.) Korovin, 1959, in Vvedensky (ed.), Fl. Uzbekist. 4: 370.

Type: UZBEKISTAN. "Asia Media, PamiroAlai, reservatum Guralasch, in Juniperetum clivi septentrionalis, 29 VI 1936, Karczevsky" (holotype LE! [LE01065977]).

- Seseli fasciculatum auct. non Korovin: Kaschtsch. 1959, in Vvedensky (ed), Fl. Kirgiz. SSR 8: 69, p. p.

Distribution in MA \& K: Kyrgyzstan (Batken prov.), Tajikistan (Sughd prov., Dushanbe prov.), Uzbekistan (Dzhizak prov., Qashqadarya prov., Navoi prov., Samarqand d.). Endemic.

Distribution area: South-Western Asia.
Seseli korshinskyi (Schischk.) Pimenov, 1977, Byull. Moskovsk. Obshch. Isp. Prir. Otd. Biol. 82(3): 141.

三 Pimpinella korshinskyi Schischk. 1950, Bot. Mater. Gerb. Bot. Inst. Komarova Akad. Nauk SSSR 13: 160.

Type: KYRGYZSTAN. "Riv. Karakul, declivum lapidosum, 4500, 18 VIII 1895, Korshinsky" (holotype LE! [LE01065707]).

Distribution in MA \& K: Kyrgyzstan (Batken prov., Jalal-Abad prov., Naryn prov.). Endemic.

Distribution area: South-Western Asia.

Seseli ledebourii G. Don, 1834, Gen. Hist. 3: 308.

ESeseli hippomarathrum L. var. hebecarpum DC., 1830, Prodr. (A. P. de Candolle) 4: 144.

Type: KAZAKHSTAN. ["In Sibiria. Adams"] "Altai, in dry grassy places at the river Irtysch, near Loktevsk and Smejov. Ledebour" LE (designated as "t ypus" by Pimenov (1978: 1960).

$=$ Elaeopleurum monococcum Korovin, Truby1962, Trudy Inst. Bot. Akad. Nauk Kazakh. SSSR 13: 248.

Type: KAZAKHSTAN. „Desertum Betpakdala (pars borealis), vall. fluv. Sarysu, in regione riv. Unrek, ad collium declivitates petraeas, 16 VI 1932, Pasyi 429" (lectotype TASH! [TASH002332], isolectotype TASH! [TASH002333]).

- Seseli hippomarathrum auct. non L.: Ledeb., 1829, Fl. Altaic. (Ledebour) 1: 335.

Distribution in MA \& K: Kazakhstan (W: W Kazakhstan prov., Aktobe prov.; N: Kostonai prov., Akmola prov., N Kazakhstan prov., Pavlodar prov.; NE: E Kazakhstan prov.; Central: Karagandy prov.).

Distribution in other Asian countries: Russia.

Distribution area: Asia (Northern and Central), Europa.

Seseli lehmannianum (Bunge) Boiss., 1872, Fl. Orient. (Boissier) 2: 967.

$\equiv$ Libanotis lehmanniana Bunge, 1852, Beitr. Fl. Russl. (Lehm. Rel. Bot.): 124; 1854, Mem. Acad. Imp. Sci. St.-Petersbourg Divers Savans 7: 300.

Type: UZBEKISTAN. "Auf felsigen Abhängen in der subalpinen Region des Karatau. [In declivibus ruperstibus regionis subalpina montium Karatau], 12-17 IX 1841, Lehmann 530" (lectotype P! [P02496296]; designated here; isolectotype LE!).

= Seseli fedtschenkoanum Regel et Schmalh. var. kokanicum Regel et Schmalh., 1881, Izv. Obshch. Ljubit. Estestv. 34(2) (Descr. Pl. Nov. Rar. Fedtsch.): 30. 
= Seseli kokanicum (Regel et Schmalh.) Lipsky ex B. Fedtsch., 1915, Rastit. Turkest.: 616.

Type: TAJIKISTAN. "In Kokaniae valle fluvii Sarawschan, inter Obburden et Pachud 5-6000' alt., O. A. Fedtschenko" (syntype LE! [LE01065973]); "Ad lacum Iskander 7000' alt., 15 VI 1870. O. A. Fedtschenko" (syntype LE! [LE01065974]); KYRGYZSTAN. "Inter Karakasuk et Schagimardan 5-6000' alt., O. A. Fedtschenko" (syntype LE); "In pratis ad fluvium Kisil-su 8000' alt., O. A. Fedtschenko" (syntype LE); "Prope oppidum Osch, 2000' alt., 06 VIII 1871, O. A. Fedtschenko" (lectotype LE! [LE01065972]; designated by V. M. Vinogradova (1997b: 60); isolectotype K [K000697452], LE! [LE01065975]).

Distribution in MA \& K: Kyrgyzstan (Batken prov.), Tajikistan (Sughd prov., Dushanbe prov.), Uzbekistan (Qashqadarya prov., Samarqand prov., Surxondarya prov., Toshkent prov.). Endemic.

Distribution area: South-Western Asia.

Seseli libanotis (L.) W. D. J. Koch, 1824, Nova Acta Phys.-Med. Acad. Caes. Leop.-Carol. Nat. Cur. 12(1): 111, fig. 49, 50.

$\equiv$ Athamanta libanotis L., 1753, Sp. P1.: 244.

三Libanotis montana Crantz,1767, Stirp. Austr. Fasc. 3: 117.

$\equiv$ Ammi daucifolium Scop., 1771, Fl. Carniol. (ed. 2) 1: 207.

三Athamanta collina Salisb., 1796, Prodr. stirp. Chap. Allerton: 168.

$\equiv$ Bubon libanotis (L.) Dumort., 1827, Fl. Belg. (Dumortier): 79.

$\equiv$ Libanotis vulgaris DC., 1830, Prodr. (A. P. de Candolle) 4: 150.

$\equiv$ Athamanta riviniiana Host ex Steud., 1840,

Nomencl. Bot., ed. 2, 1: 166.

$\equiv$ Seseli vulgare (DC.) Bubani, 1900, Fl. Pyren.

(Bubani) 2: 376, nom. illeg. (Art. 52.1).

$\equiv$ Selinum libanotis (L.) E. H. L. Krause, 1904, in

Deutschl. Fl. (Sturm), ed. 2, 12: 102, tab. 18.

三Balinotella libanotis (L.) Soják, 1982, Zprávy

Krajsk. Vlastiv. Muz. Olomouci 215: 3.

Type: EUROPE. "In Sueciae, Germaniae pratis siccis apricis, 345.1" (lectotype LINN [LINNHL345-1], designated by Pardó (1981: 168)).

= Libanotis daucoides Scop., 1771, Fl. Carniol. (ed. 2) 1: 192.

= Athamanta daucoides (Scop.) J. F. Gmelin, 1791, Syst. Nat. ed. 13 2(1): 477.

= Athamanta daucifolia Host, 1827, Fl. Austriac. 1: 362 .

= Libanotis daucifolia (Host) Rchb. 1832, Fl. Germ. Excurs.: 468.
Type: AUSTRIA. "[Carniolia] In alpibus Vochinensibus".

= Athamanta pubescens Retz., 1783, Obs. Bot. 3: 28 .

= Ligusticum athamantoides Spreng., 1813, Neue Schr. Naturf. Ges. Halle 2(1) (Pl. Umb. Prodr.): 40.

= Seseli athamantoides Ledeb., 1829, Fl. Altaic. (Ledebour) 1: 342.

= Libanotis sibirica C. A. Mey., 1831, Verz. Pfl. Casp. Meer. (C. A. von Meyer): 124.

Type: RUSSIA? "[Altai] In campestribus ubique, Ledebour" (syntype LE?); KAZAKHSTAN "Ad fl. Irtysch, 14 VIII 1826, Meyer 219" (lectotype LE! [LE01065737], designated by V. M. Vinogradova (2005: 965, isolectotype LE! [LE01065738)).

$=$ Seseli athamantoides Ledeb. var. acaule Ledeb., 1829, Fl. Altaic. (Ledebour) 1: 342.

Type: KAZAKHSTAN. "Ad rivulum Tschaganka prope mont. Tschingistan deserti soongoro-kirghisici locis fertilibus subhumidis, 14 VIII 1826, Meyer 219" (lectotype LE [LE01065740], designated by V. M. Vinogradova (2005).

= Libanotis intermedia Rupr., 1845, Beitr. Pflanzenk. Russ. Reiches 4: 53.

= Seseli libanotis (L.) W. D. J. Koch subsp. intermedium (Rupr.) P. W. Ball, 1968, Feddes Repert. 79(1-2): 64.

= Seseli intermedium (Rupr.) Vodopyanova, 1979, in Malyshev (ed.), Fl. Tsentral'noi Sibiri 2: 681, fig. 23.2, map 965.

Type: EUROPA, RUSSIA. "In umbrosis ad pedem montis Duderhof, 1842, Zobel" (holotype LE!).

= Seseli sibiricum Garcke, 1849, Fl. N. Mitt. Deutschland (ed. 1): 139.

- Libanotis sibirica auct. non C. A. Mey.: Krylov, 1935, Fl. Zapadnoi Sibiri 8: 2069.

- Libanotis transcaucasica auct. non Schischk.: Achundov, 1955, Fl Azerb. S.S.R.: 464, p. p.

- Seseli transcaucasucum auct. non Pimenov et

Sdobnina: Manden. 1934, Fl. Georg. 2 ed. 9: 231.

Distribution in MA \& K: Kazakhstan (W: W Kazakhstan prov., Aktobe prov.; N: Kostonai prov., Akmola prov., N Kazakhstan prov., Pavlodar prov.; NE: E Kazakhstan prov.; Central: Karagandy prov.).

Distribution in other Asian countries: Russia, China, Iran, Azebraydzhan, Georgia, Armenia, Turkey.

Distribution area: Asia (Northern, Central, South-Western and Mediterranean), Europa, Africa, N. America (adv.). 
Seseli luteolum Pimenov, 2002, Bot. Zhurn. (St. Petersburg) 87(6): 135.

Type: KYRGYZSTAN. "Kyrgyzstan, TianSchan centralis, in valle fl. Kokomeren, infra pagum Ksyl-oi, in declivibus lapidosis, 24 VII 1970, Pimenov \& Borjaev 807" (lectotype LE! [LE01065984], designated by Pimenov (1978: 192); isolectotypes LE! [LE01065985-LE01065988], MW! [MW0593869, MW0593870]).

- Seseli fasciculatum auct. non Korovin: Schischk., 1950, in Schischkin (ed.), Fl. URSS 16: 507, p. p., quoad pl. Tiansch. centr.

Distribution in MA \& K: Kyrgyzstan (JalalAbad prov., Naryn prov.). Endemic.

Distribution area: South-Western Asia.

Seseli marginatum (Korovin) Pimenov et Sdobnina, 1973, Bot. Zhurn. (Leningrad) 58(10): 1490.

$\equiv$ Libanotis marginata Korovin, 1924, Bot. Mater. Gerb. Glavn. Bot. Sada R.S.F.S.R. 5(6): 81.

Type: KAZAKHSTAN. "Prov. Syr-Darja, distr. Chimkent, in parte superiore system. fl. Aksu, regio stepposa, alt. ca. 4000', in clivis conglomeratis, 14 VIII 1921, Abolin \& Popov 8275" (lectotype TASH! [TASH002323], designated by Pimenov (1978: 189); "Prov. Syr-Darja, dist. Aulie-ata, finis meridianam Kara-tau. 11 IX 1921. Abolin \& Popov 8953" (syntypes TASH [TASH002321, TASH002322]).

= Seseli karatavicum Schischk., 1950, Bot. Mater. Gerb. Bot. Inst. Komarova Akad. Nauk SSSR 13: 163 .

Type: KAZAKHSTAN. „Kazachstania, jugum Kara-dak in rupestribus sub cacumine montis Bucun-tau, 12 IX 1931, N.V. Pavlov 1260" (holotype LE! [LE01065970]).

Distribution in MA \& $\mathbf{K}$ : Kazakhstan ( $\mathrm{S}: \mathrm{S}$ Kazakhstan prov., Zhambyl prov.), Uzbekistan (Toshkent prov.). Endemic.

Distribution area: South-Western Asia.

Seseli merkulowiczii (Korovin) Pimenov et Sdobnina, 1975, Bot. Zhurn. (Leningrad) 60(8): 1121.

$\equiv$ Libanotis merkulowiczii Korovin, 1947, Not. Syst. Herb. Inst. Bot. Zool. Acad. Sci. Uzbekistan 8: 16.

Type: UZBEKISTAN. "Pamir-Alaj, montes Hissarici, vallis fl. Surchan-Darja, ad pag. Churbaton, 05 VIII 1931, Merkulowicz" (holotype TASH! [TASH002325, TASH002326]).

Distribution in MA \& K: Uzbekistan (Surxondarya prov.). Endemic.

Distribution area: South-Western Asia
Seseli mironovii (Korovin) Pimenov et Sdobnina, 1973, Bot. Zhurn. (Leningrad) 58(10): 1489.

三Libanotis mironovii Korovin, 1962, Trudy Inst. Bot. Akad. Nauk Kazakh. SSR 13: 252.

Type: KAZAKHSTAN. "Desertum BetpakDala, monticola Tanatkan, ad declivitatem lapidosum, 03 IX 1965, Mironov 942, 943” (holotype TASH).

Distribution in MA \& K: Kazakhstan (Central: Karagandy prov.; S: Zhambyl prov.). Endemic.

Distribution area: Central Asia.

Seseli mucronatum (Schrenk) Pimenov et Sdobnina, 1973, Byull. Moskovsk. Obshch. Isp. Prir. Otd. Biol. 78(4): 139.

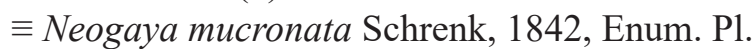
Nov. 2: 40; 1843, Bull. Phys.-Math. Acad. Petersb. 1: 80 .

$\equiv$ Pachypleurum mucronatum (Schrenk) Schischk. 1950, in (ed.), Fl. URSS 16: 581, tab. 34, 37.

$\equiv$ Ligusticum mucronatum (Schrenk) Leute, 1970, Ann. Naturhist. Mus. Wien 74: 473. 1970

Type: KAZAKHSTYAN "In montibus Alatau [Songarei, Alatau], 18 VII 1841, Schrenk 850" (lectotype LE! [LE01065785]; designated as type by Pimenov \& Sdobnina (1973: 139); isolectotypes LE! [LE01065786-LE01065789].

=Ligusticum thomsonii C. B. Clarke, 1879, in Fl. Brit. India (J. D. Hooker) 2: 698.

Type: INDIA. "Tibet occid., Reg. temp. Zanskar, 11000-13000 ped., 29 VI 1848, Thomson" (syntypes BM, G! [G00367247], K [K000695853, K000695854]); "Tibet occ. Reg. temp. alt. 1113000 ped., Nubra, 01 VIII 1848, Thomson, 2 Oreocome" (lectotype E! [E00265213], designated by Leute (1970: 490); isolectotypes E [E00265213], G! [G00367248], K! [K000695851, K000695852], MH!, NY [NY00406027], P! [P03221130P03221132]).

= Pleurospermum longicaule H. Wolff, 1929, Repert. Spec. Nov. Regni Veg. 27: 117.

Type: CHINA. Kansu: upper Kap Ching K'ou, near Old Taochow, 3800-4300 m s. m., 28-31 VIII 1923, Ching 878"' (holotype US! [US00036517]).

$=$ Libanotis subsimplex Popov, 1935, Index Sem. Hort. Bot. Alma-Ata 2: 13; 1957, Not. Syst. Herb. Inst. Bot. Acad. Sci. URSS 18: 318.

Type: KAZAKHSTAN. [Alatau transiliensis] "Mts. Kungei Alatau, ad fontes fluminis Tschilik, declivia pratensia in valle rivuli Tjulku-bulak, 17 VIII 1934, Popov" (neotype AA!; designated by V. P. Goloskokov (1963: 30). 
$=$ Libanotis dolichostyla Schischk., 1950, in Schischkin (ed.), Fl. URSS 16: 600, 480.

= Pachypleurum dolichostylum (Schischk.) Korovin ex Kamelin, 1971, in Fl. Rastit. Uchel. Reki Varsob: 194.

= Seseli dolichostylum (Schischk.) M. Hiroe, 1979, Umbelliferae World: 1126, "dolichostyla".

Type: KYRGYZSTAN. "Asia Media, Montes Tjan-Schan. Ad ripam fl. Karakol occident., 22 VII 1908, Roshevitz" (holotype LE! [LE01066035]).

Distribution in MA \& K: Kazakhstan (S: S Kazakhstan prov.; SE: Almaty prov.), Kyrgyzstan (Jalal-Abad prov., Ysyk-köl prov., Naryn prov., Osh prov., Talas prov., Chüh prov..), Tajikistan (Kŭhistani Badakhshan, Sughd prov., Dushanbe prov.), Uzbekistan (Dzhizak prov., Qashqadarya prov., Toshkent prov.),

Distribution in other Asian countries: China, India, Pakistan, Mongolia, Afghanistan

Distribution area: Central, Eastern and SouthWestern Asia

Note: The type of $L$. subsimplex was not specified in the protologue (Goloskokov, 1963); in this regard, a neotype could be proposed from the specimens stored in AA.

Seseli nemorosum (Korovin) Pimenov, 1979, Novit. Syst. Pl. Vasc. 15: 200.

$\equiv$ Neogaya nemorosa Korovin, 1947, Bot. Mater. Gerb. Inst. Bot. Zool. Akad. Nauk Uzbeksk. SSR 8: 12.

$\equiv$ Pachypleurum nemorosum (Korovin) Korovin, 1959, in Vvedensky (ed.), Fl. Uzbekist. 4: 389.

Type: KYRGYZSTAN. "Tian-Schan, montes Talassici, in valle fl. Arabik, 08 IX 1923, Korovin 155" (holotype TASH! [TASH002361]).

= Pachypleurum talassicum Bajtenov, 1971, Bot. Mater. Gerb. Inst. Bot. Akad. Nauk Kazakh. SSR 7: 29.

Type: KAZAKHSTAN. "Alatau Talassicus, fl. Dzhebogly, in declivibus lapidosis 02 VII 1962. Karmyscheva" (holotype AA!).

Distribution in MA \& K: Kazakhstan (S: S Kazakhstan prov.), Kyrgyzstan (Jalal-Abad prov., Talas prov.), Uzbekistan (Toshkent prov.). Endemic.

Distribution area: South-Western Asia.

Seseli nevskii (Korovin) Pimenov et Sdobnina, 1973, Bot. Zhurn. (Leningrad) 58(10): 1489.

$\equiv$ Libanotis nevskii Korovin, 1947, Not. Syst. Herb. Inst. Bot. Zool. Acad. Sci. Uzbekistan 8: 19.

Type: TURKMENISTAN. "Pamir-alaj, montes Kuhitang, ad pag. Chodscha-fil-ata, 25 VII 1935, Androssov 613"' (holotype ASH!).
Distribution in MA \& K: Uzbekistan (Surxondarya d,), Turkmenistan (Lebap prov.). Endemic.

Distribution area: South-Western Asia.

Note: N 613 with above mentioned label is indicated in protologue. There is, however, in ASH only a specimen with different label and number: "Karluk district, Kuhitang Mts., in roch crevices in gorge near Chodzha-fil-ata. 27 VII 1935. Androssov $1751 "$.

Seseli pelliotii (H. Boissieu) Pimenov et Kljuykov, 2000, Bot. Zhurn. (St. Perersburg) 85(10): 105, fig. 2, E.

$\equiv$ Pituranthos pelliotii H. Boissieu, 1910, Bull. Mus. Hist. Nat. (Paris) 16: 164.

$\equiv$ Eriocycla pelliotii (H. Boissieu) H. Wolff, 1927, in Pflanzenr. (Engler) Umbellif.-Apioid.-Ammin. 90(IV, 228): 106.

Type: CHINA. "Turkestan chinois, prov. de Kou-Tchar, vallée de Gair, à 1500 m. 03 VIII 1907. Pelliot \& Vaillant 436" (holotype P! [P00752857]).

= Platytaenia depauperata Schischk., 1951, in Schischkin (ed.), Fl. URSS 17: 356, 270.

= Semenovia depauperata (Schischk.) Manden., 1959, Trudy Tbilis. Bot. Inst. 20: 22.

= Zosima depauperata (Schischk.) M. Hiroe, 1979, Umbelliferae World: 1761.

= Seseli depauperatum (Schischk.) V. M. Vinogr. 1981, in S. K. Cherepanov, Sosud. Rast. SSSR: 30.

Type: KYRGYZSTAN. "Kyrgyzstan, Montes Tian-Schan, ad ripas Kiczik-Usungusch, 4 km supra ostium, in clivis herbosis lapidosisque, 01 IX 1939, Jakovleva 235" (holotype LE! [LE00052225]).

Distribution in MA \& K: Kyrgyzstan (Ysykköl prov., Naryn prov.).

Distribution in other Asian countries: China.

Distribution area: Central Asia.

Seseli rimosum Pimenov, 1977, Byull. Moskovsk. Obshch. Isp. Prir. Otd. Biol. 82(3): 139.

Type: TAJIKISTAN. "Tadshikistania, area naturalis hissaro-darvasica, declivium australe jugi Petri Primi, vallis fl. Obi-Chingou, in fissuris rupium prope pag. Punjekva, 24 VII 1974, Pimenov 624" (holotype LE! [LE01065992]; isotypes MW! [MW0593877-MW0593883]).

Distribution in MA \& K: Tajikistan (Khatlon prov., Dushanbe prov.). Endemic.

Distribution area: South-Western Asia.

Seseli schrenkianum (C. A. Mey. ex Schischk.) Pimenov et Sdobnina, 1975, Bot. Zhurn. (Leningrad) 60(8): 1119 . 
$\equiv$ Libanotis schrenkiana C. A. Mey. ex Schischk., 1950, in Schischkin (ed.), Fl. URSS 16: 601, 478.

$\equiv$ Balinotella schrenkiana (C. A. Mey. ex Schischk.) Soják, 1982, Zprávy Krajsk. Vlastiv. Muz. Olomouci 215: 4.

Type: KAZAKHSTAN. "[described on the basis of plants, grown in St. Petersburg Botanical Garden from seeds, collected by A. Schrenk in East Kazakhstan] (holotype LE! [LE01066054]).

- Libanotis sibirica auct. non C. A. Mey.: O. et B. Fedtsch., 1909, Consp. Fl. Turkest. (O. A. Fedtschenko et B. A. Fedtschenko) 3: 95.

Distribution in MA \& K: Kazakhstan (S: S Kazakhstan prov., Zhambyl prov.; SE: Almaty prov.), Kyrgyzstan (Jalal-Abad prov. Ysyk-köl prov., Naryn prov., Osh prov., Talas prov., Chüh prov.), Tajikistan (Kŭhistani Badakhshan, Sughd prov., Dushanbe prov.), Uzbekistan (Dzhizak prov., Qashqadarya prov., Samarqand prov., Surxondarya prov., Toshkent prov.).

Distribution in other Asian countries: China.

Distribution area: Central and South-Western Asia.

Seseli sclerophyllum Korovin, 1975, Izv. Akad. Nauk Tadzh. SSR, Otd. Estest. Nauk 3(60): 7.

Type: TAJIKISTAN."Ad declivitatem australem jugi Darvasici, in valle fl. Obi-Charok, 09 VII 1962, N. G. Popov, Stepanenko \& Statzkji 1222" (holotype TAD!).

Distribution in MA \& K: Tajikistan (Kŭhistani Badakhshan, Dushanbe prov.). Endemic.

Distribution area: South-Western Asia.

Note: Lately, despite special search, the species was not collected in its classical locality.

Seseli seravschanicum Pimenov et Sdobnina, 1975, Bot. Zhurn. (Leningrad) 60(8): 1118.

$\equiv$ Ligusticum fedtschenkoanum Schischk. 1950, Bot. Mater. Gerb. Bot. Inst. Komarova Akad. Nauk SSSR 13: 166, non Regel et Schmalh. (1882).

Type: TAJIKISTAN. "Asia Media, fontes $\mathrm{fl}$. Seravschan, ad ripam lacus Karakul, 30 VII 1892, Komarov" (holotype LE! [LE01065783]).

Distribution in MA \& K: Tajikistan (Sughd prov.), Uzbekistan (Qashqadarya prov., Surxondarya prov.). Endemics.

Distribution area: South-Western Asia.

Seseli sessiliflorum Schrenk, 1845, Bull. Phys.Math. Acad. Petersb. 3: 307.

Type: KAZAKHSTAN. "In montibus Chantau, locis arenosis, 24 VI 1843, Schrenk" (lectotype LE!
[LE01066002]; designated as "typus" by Pimenov (1978: 195); isolectotypes LE! [LE01066003LE01066005], P! [P02496637]).

= Seseli squarrosum Schischk. 1950, Bot. Mater. Gerb. Bot. Inst. Komarova Akad. Nauk SSSR 13: 162.

Type: KAZAKHSTAN. "Kasachstania, distr. Karsakpai, ad marginem occidentalem deserti Bedpak-dala, in loco Kamys-bulak, 14 VIII 1929, Smirnov 622" (lectotype LE! [LE01066007], designated by V. M. Vinogradova (1997: 60); "Kzyl-Orda prov., Karsakpai distr. W Betpadala. 14 VIII 1929. Smirnov 522" (syntype LE! [LE01066008]) VIII 1929, Smirnov 622" (holotype LE!).

Distribution in MA \& K: Kazakhstan (Central: Kzyl-Orda prov.; S: S Kazakhstan prov., Zhambyl prov.; SE: Almaty prov.), Kyrgyzstan (Jalal-Abad prov., Ysyk-köl prov., Chüh prov.).

Distribution in other Asian countries: China.

Distribution area: Central and South-Western Asia.

Seseli setiferum Pimenov et Sdobnina, 1975, Bot. Zhurn. (Leningrad) 60(8): 1118.

इLigusticum setiferum Korovin ex Pavlov, 1937, Trudy Prikl. Bot., ser. 1, Sist. Rast. Obscie Vopr. Rasteniev.: 267, nom. inval. (Art. 39.1), sine descr.

$\equiv$ Libanotis setifera (Korovin ex Pavlov) Schischk., 1950, in Schischkin (ed.), Fl. URSS 16: 481, nom. inval. (Art. 39.1), descr. ross.

Type: KAZAKHSTAN. "Montes Talass-Alatau, in fissuris saxorum in fluvii superiore fl. Dzhabaglysu, 09 VIII 1931, N.V. Pavlov 992" (holotype MW! [MW0593887]).

Distribution in MA \& K: Kazakhstan (S: S Kazakhstan prov.), Uzbekistan (Toshkent prov.). Endemic.

Distribution area: South-Western Asia.

Seseli staurophyllum Rech. f. 1940, Repert. Spec. Nov. Regni Veg. 48: 127.

$\equiv$ Eriocycla staurophylla (Rech. f.) Rech. f., Riedl et Kuber 1964, Anz. Oesterr. Akad. Wiss. Math.-Naturwiss. K1. 101(1-15): 365.

$\equiv$ Lomatopodium staurophyllum (Rech. f.) Rech. f. 1987, in Flora Iranica (Rechinger) 162: 349, tab. 281.

Type: IRAN. "Khorassan: in montibus serpentinicis prope Robat-i Safid inter Mashhad et Torbate Hayderi, 1700 m, 10 VII 1937, Rechinger 1510" (lectotype W! [W1951-0011600]; designated by Rechinger (1987: 349), as "typus"; isolectotypes BM [BM000944753], K! [K000697449], NY [NY00406273], US! [US00127231]). 
= Seseli jomuticum Schischk. 1950, Bot. Mater. Gerb. Bot. Inst. Komarova Akad. Nauk SSSR 13: 163.

Type: TURKMENISTAN. "Asia Media, Turcomania, in alveo sicco rivi montani ad stationem viae ferreae Achcza-Kujma, 21 X 1915, Androssov 1578" (holotype LE! [LE01065966]).

Distribution in MA \& K: Kazakhstan (W: Aktobe prov., Mangystau prov.), Uzbekistan (Karakalpak), Turkmenistan (Balkan prov.).

Distribution in other Asian countries: Iran.

Distribution area: Central and South-Western Asia.

Note: The species is closely related to S. cuneifolium M. Bieb. and S. eriocephalum (Pall.) B. Fedtsch., forming a complicated taxonomic circle. Their united area is very large - from Eastern Caucasus to Kazakhstan-Chinese border. Although infrapopulational and interpopulational variability is sometimes broad, it is difficult to find any constant differences among long-distant populations. Analysis of nrDNA ITS variation (unpublished data) also does not show any difference among them. S. staurophyllum (incl. S. jomuticum) seems now to be differing from $S$. eriocephalum in its polycarpic (not monocarpic) life-form.

Seseli strictum Ledeb., 1829, Fl. Altaic. (Ledebour) 1: 338.

$\equiv$ Athamanta stricta (Ledeb.) Ledeb., 1840, in Nomencl. Bot. (Steudel), ed. 2, 1: 166.

Type: RUSSIA/KAZAKHSTAN. "In pratensibus ad radices montium altaicorum frequens $v$. gr. prope Sogra, Loktewsk, Smejow alibique (Ledebour \& Meyer); [Loktewsk], Ledebour \& Meyer 257" (lectotype LE!, designated as "typus" by Pimenov (1978: 197); isolectotypes C! [C10008566?], P! [P02496631]; “Altai, Sogra, 24 VIII 1826, Ledebour 1151” (syntype LE! [LE01066013]).

= Pseudammi ehrenbergii H. Wolff, 1920, Repert. Spec. Nov. Regni Veg. 17: 173.

= Ammi ehrenbergii (H. Wolff) M. Hiroe, 1979, Umbelliferae World: 1025.

Type: RUSSIA. "Sibiria occidentalis: inter Omsk et Petropavlowsk, 1929, Ehrenberg" (syntype); "Altai: loco et tempore non indicates, Ehrenberg" (syntype).

Distribution in MA \& K: Kazakhstan (W: Aktobe prov.; N: Kostonai prov., Akmola prov., N Kazakhstan prov., Pavlodar prov.; NE: E Kazakhstan prov.; Central: Karagandy prov.; S: Dzhambyl prov.).

Distribution in other Asian countries: Russia, China.
Distribution area: Asia (Northern and Central), Europa (E).

Seseli talassicum (Korovin) Pimenov et Sdobnina, 1975, Bot. Zhurn. (Leningrad) 60(8): 1118.

$\equiv$ Ligusticum pumilum Korovin, 1924, Bot. Mater. Gerb. Glavn. Bot. Sada R.S.F.S.R. 5(6): 82, nom. illeg. (Art. 53.1), non A. P. de Candolle (1830: 245).

三 Ligusticum talassicum Korovin, 1979, in Vvedensky (ed.), Fl. Uzbekist. 4: 384, excl. distr.

$\equiv$ Ligusticum turkestanensis M. Hiroe,1979, Umbelliferae World: 1088, nom. illeg. (Art 52.1).

Type: KAZAKHSTAN. "Mont. Ak-baschtau, in valle Dondus, 12 VIII 1921, Abolin \& Popov 8258" (lectotype LE! [LE01065784TASH! [TASH002359]; designated by V. M. Vinogradova (1997b: 54); isolectotypes TASH! [TASH! [TASH002359, TASH002360]).

Distribution in MA \& K: Kazakhstan (S: S Kazakhstan prov.), Kyrgyzstan (Jalal-Abad prov., Naryn prov., Talas prov.), Uzbekistan (Toshkent prov.). Endemic.

Distribution area: South-Western Asia.

Seseli tenellum Pimenov, 1974, Bot. Zhurn. (Leningrad) 59(3): 373.

Type: KYRGYZSTAN. "Kyrgyzstan, TianSchan occidentalis, in valle fl. Chatkal infra pag. Jangi-Bazar, saxa et declivia lapidosa adversum ostium fl. Ters, 01 VIII 1970, Pimenov 998" (holotype LE! [LE01066014]; isotype LE! [LE01066015]).

Distribution in MA \& K: Kyrgyzstan (JalalAbad prov.). Endemic.

Distribution area: South-Western Asia.

Seseli tenuisectum Regel et Schmalh., 1882, Izv. Obshch. Ljubit. Estestv. 34(2) (Descr. Pl. Nov. Rar. Fedtsch.): 31 .

$\equiv$ Libanotis tenuisecta (Regel et Schmalh.) Korovin, 1963, in Pavlov (ed.), Fl. Kazakhst. 6: 345.

Type: KYRGYZSTAN. "In Kokania, in trajectu Kendyr-davan SW Alatau, 25 VIII 1871, O. A. Fedtschenko" (lectotype LE! [LE01066029], designated as type by Pimenov (1974: 243;1978: 190); "In Turkestaniae montibus alatavicis in valle fl. Tschirtschik, 4-6000', VIII 1876, A. Regel" (syntype LE! [LE01066027, LE01066030]); "In valle fl. Tschotkal, 4-6000', VIII 1876, A. Regel" (syntypes K! [K000697455], LE! [LE01066028, LE01066031]).

= Libanotis juncea Korovin, 1947, Not. Syst. Herb. Inst. Bot. Zool. Acad. Sci. Uzbekistan 8: 17. 
Type: TAJIKISTAN. "Tian-schan, montes Mogoltavici, 18 VI 1933, B. M. Komarov" (holotype Herb. Khudzhand!).

- Seseli giganteum auct. non Lipsky: Schischk., 1950, in Schischkin (ed.), Fl. URSS 16: 509, p. p.

Distribution in MA \& K: Kazakhstan (S: S Kazakhstan prov.), Kyrgyzstan (Batken prov., JalalAbad prov.), Tajikistan (Sughd prov.), Uzbekistan (Namangan prov., Toshkent prov.). Endemic.

Distribution area: South-Western Asia.

Seseli turbinatum Korovin, 1947, Not. Syst. Herb. Inst. Bot.Zool. Acad. Sci. Uzbekistan 8: 21.

Type: UZBEKISTAN. "Pamir-alaj meridionalis, jugum Koj-tasch, m. Piasly, 05 VIII 1934 VIII 1931, Botschanczev" (holotype TASH! [TASH002317]).

Distribution in MA \& K: Kazakhstan (S: S Kazakhstan prov.), Tajikistan (Sughd prov.,), Uzbekistan (Dzhizak prov.). Endemic.

Distribution area: South-Western Asia.

Seseli unicaule (Korovin) Pimenov, 1974, Novit. Syst. P1. Vasc. 11: 245.

$\equiv$ Libanotis unicaulis Korovin, 1947, Not. Syst. Herb. Inst. Bot. Zool. Acad. Sci. Uzbekistan 8: 18.

Type: KYRGYZSTAN. "Tian-Schan, montes Ferganici, vallis riv. Tochtalyk, 03 IX 1927, Abolin 613" (lectotype TASH! [TASH002328, TASH002329], designated as type by Pimenov, 1974: 245; isolectotype TASH! [TASH002330]).

- Seseli lehmannianum auct. non Boiss.: Schischk., 1950, in Schischkin (ed.), Fl. URSS 16: 506, p. p.

- Seseli giganteum auct. non Lipsky: Kaschtsch. 1959, in Vvedensky (ed.), Fl. Kirgh. SSR 8: 70.

Distribution in MA \& K: Kazakhstan (S: S Kazakhstan), Kyrgyzstan (Jalal-Abad prov., Naryn prov., Osh prov., Talas prov.), Uzbekistan (Fergona prov.). Endemic.

Distribution area: South-Western Asia.

Seseli vaillantii H. Boissieu, 1910, Bull. Mus. Natl. Hist. Nat. (Paris) 16: 165.

Type: CHINA. "Turkestan chinois, prov. de Kutchar, Zamutek-Tagh, mine d'Ammone, à 2000 m. [Asie Centrale, Zamutek tagh (Ammone)], 30 VII 1907, Pelliot \& Vaillant 382" (holotype P! [P02496432, P02496433]).

= Seselifedtschenkoanum Regel et Schmalh. var. iliense Regel et Schmalh., 1882, Izv. Obshch. Ljubit. Estestv. 34(2) (Descr. Pl. Nov. Rar. Fedtsch.): 31.
$=$ Seseli iliense (Regel et Schmalh.) Lipsky ex

B. Fedtsch., 1915, Rastit. Turkest.: 616.

$=$ Libanotis iliensis (Regel et Schmalh.) Korovin, 1963, in Pavlov (ed.), Fl. Kazakhst. 6: 345.

Type: KAZAKHSTAN. "In valle fl. Ili in angustis Koibin, 1876, A. Regel" (lectotype LE! [LE01065963], designated by Pimenov (1978: 190); isolectotypes K! [ K000697451], LE! [LE01065965], P [P02496318); "Ilithal, 1876. A. Regel" (syntype LE! [LE01065964]).

= Seseli altissimum Popov, 1940, Bot. Mat. Gerb. Bot. Komarova Akad. Nauk SSSR 8(4): 72.

Type: KAZAKHSTAN. "Tianschan orientalis, regio iliensis, in angustiis fluminis Tscharyn loco Kurtogai dicto, in rupibus porphyricis, 12 VIII 1937, Popov \& Goloskokov" (holotype AA!; isotype LE! [LE01065941, LE01065942, LE01065943]).

Distribution in MA \& $\mathbf{K}$ : Kazakhstan (SE: Almaty prov.).

Distribution in other Asian countries: China.

Distribution area: Central and South-Western Asia.

Seseli valentinae Popov, 1940, Bot. Mat. Gerb. Bot. Inst. Komarova Akad. Nauk SSSR 8(4): 73.

Type: KAZAKHSTAN. "Tianschan orientalis, jugum Kungei Alatau ad flumen Tschilik ad rivulum Saty, in collibus siccis rubris gypsaceis, 22 VIII 1937, Michailova" (lectotype LE!, designated by V. P. Goloskokov, 1963: 53; isolectotype AA!).

$=$ Lomatopodium lessingianum (Turcz.) Fisch. et C. A. Mey. var. tenuifolium Regel et Herder, 1866, Bull. Soc. Imp. Naturalistes Moscou 39(3): 75.

Type: KAZAKHSTAN. "Keskelen-Thale im Alatau transiliensis, Semenov" (holotype LE! [LE01066033]; isolectotype LE! [LE01066073]); "Kungei Alatau, riv. Tschilik, angustis riv. Saty 14 VII 1937. Michailova" (syntype LE! [LE01066034]).

Distribution in MA \& K: Kazakhstan (SE: Almaty prov.), Kyrgyzstan (Ysyk-köl prov., Naryn prov.).

Distribution in other Asian countries: China.

Distribution area: Central and South-Western Asia.

Seselopsis pusilla Pimenov et Lavrova, 1983, Bot. Zhurn. (Leningrad) 68(11): 1565, fig. 2.

Type: KYRGYZSTAN. "Kyrgyzstan, pugum Alaicum in systemate fluminis Gulcza, in declivibus gypsaceis inter lacum Chonkol et pagum Kolduk, 25 VII 1981, Pimenov et al. 709" (holotype LE! [LE01065773]; isotypes E! [E00000464], 
LE [LE01065774, LE01065775], MHA!, MW! [MW0593841], TASH! [TASH002229]).

Distribution in MA \& K: Kyrgyzstan (Osh prov.).

Distribution in other Asian countries: China.

Distribution area: South-Western Asia.

Seselopsis tianschanica Schischk. 1950, Bot.

Mater. Gerb. Bot. Inst. Komarova Akad. Nauk SSSR 13: 159.

Type: KAZAKHSTAN. "Tian-Schan, jugum Ketmenj-tau in faucibus Kischkine-Czijbut, in rupium fissuris, alt. ca. $2500 \mathrm{~m}, 14$ VIII 1946, Rubtzov" (holotype LE! [LE01065776]; isotypes LE01065777, LE01065778]).

Distribution in MA \& K: Kazakhstan (SE: Almaty prov.).

Distribution in other Asian countries: China.

Distribution area: Central \& South-Western Asia.

Silaum silaus (L.) Schinz et Thell., 1915, Vierteljahrssch. Naturf. Ges. Zürich 60: 359.

$\equiv$ Peucedanum silaus L., 1753, Sp. P1.: 246.

$\equiv$ Selinum silaus (L.) Crantz, 1767, Cl. Umb.

Emend.: 61.

$\equiv$ Ligusticum silaus (L.) Vill., 1779, Prosp. Hist.

P1. Dauphiné: 25.

$\equiv$ Sium silaus (L.) Roth., 1788, Tent. Fl. Germ. 1: 129 .

$\equiv$ Peucedanum flavum Salisb., 1796, Prodr. stirp.

Chap. Allerton: 163, nom, illeg. (Art. 52.1).

$\equiv$ Crithmum silaus (L.) Wibel, 1799, Prim. Fl.

Werth.: 197.

$\equiv$ Silaus flavescens Bernh., 1800, Syst. Verz.

(Bernhardi): 174, nom, illeg. (Art. 52.1).

$\equiv$ Cnidium silaus (L.) Spreng., 1813, Neue Schr.

Naturf. Ges. Halle 2(1) (Pl. Umb. Prodr.): 40.

$\equiv$ Meum silaus (L.) Baill., 1879, Hist. Pl. (Bail-

lon) 7: 191 .

$\equiv$ Silaus silaus (L.) Karst., 1895, Deut. Fl. (ed. 2)

2: 402, nom. illeg. (Art. 23.4).

Type: EUROPE. "In Helvetia, Narbona VIII 73"

(lectotype UPS-Burser, designated by Reduron in Jonsell et Jarvis, 2002: 84).

= Peucedanum alpestre L., 1753, Sp. Pl.: 246.

= Selinum alpestre (L.) Crantz, 1767, Cl. Umb. Emend.: 60 .

$=$ Silaus alpestris (L.) Besser ex Schult., 1820, in Syst. Veg. ed. 15 bis (Roemer et Schultes) 6: 36; Besser, 1822, Enum. Pl. (Besser): 43.

= Ligusticum alpestre (L.) Calest., 1905, Webbia 1: 120.
= Silaus alpestre (L.) Thell., 1926, in Hegi (ed.), Ill. Fl. Mittel.-Eur. 5(2): 1295.

Type: "alpestre 2" 346.4" (lectotype LINN [LINN-HL346-4], designated by J.-P. Reduron in: Jarvis \& al., Taxon 55(1): 213 (2006); "Peucedanum foliolis linearibus ramosis Leiden, van Royen" (syntype L! [L0060378]).

Note: In historical herbarium of van Royen (L) there is the second specimen of Peucedanum alpestre (L0053021). It corresponds to L0060378 in all details with an exclusion - it contains a stem top with mature fruits, being evidently an alien element in this collection. L0053021 can be regarded as type material only pro parte.

$=$ Seseli selinoides Jacq., 1762, Enum. Stirp. Vindob.: 51, 227.

= Silaus selinoides (Jacq.) Halácsy, 1886, in Kerner (ed.), Sched. Fl. Austro-Hung. 4: 37.; ex Simonk., 1886, Enum. Fl. Transsilv.: 261.

Type: EUROPE, AUSTRIA. "In pratensibus inter Lanzendorf et Himberg".

$=$ Seseli pratense Crantz, 1767, Stirp. Austr. Fasc. 3: 96.

= Peucedanum pratense (Crantz) Lam., 1779, Fl. franç. 3: 469.

= Silaus pratensis (Crantz) Besser ex Spreng., 1820 , in Syst. Veg. ed. 15 bis (Roemer et Schultes) 6: 36 .

= Cnidium pratense (Crantz) Bubani, 1900, Fl. Pyren. (Bubani) 2: 381.

Type: EUROPE, AUSTRIA. Prope Lantz, Ebersdorf, in prato supra Grunzing.

= Selinum sibiricum Retz., 1781, Observ. Bot. 2: 16.

= Meum sibiricum Spreng., 1813, Neue Schr. Naturf. Ges. Halle 2, 1 (Pl. Umb. Prodr.): 32.

Type: RUSSIA. "In Sibiria" (lectotype B [B-W 05718-04 0]; designated here; isolectotypes B [B-W 0571801 0, B-W 05718-02 0, B-W 05718-03 0], LD [LD1743608]).

= Silaus besseri DC., 1830, Prodr. (A. P. de Candolle) 4: 161 .

= Silaum besseri (DC.) Grossh. 1932, Fl. Kavkaza (Grossheim) 3: 170.

Type: EUROPE, UKRAINE. "In campis apricis Podoliae australis. [In Osopo campis] 1828, Besser" (lectotype G-DC, designated by Reduron \& Jacquemoud (Reduron, 2008: 2367); isolectotype KW-Besser!, LECB [LECB0000083]).

= Silaus gracilis Bunge, 1852, Beitr. Fl. Russl. (Lehm. Rel. Bot.): 125; 1854, Mem. Acad, Imp. Sci. St.-Petersbourg Divers Savans 7: 301. 
Type: EUROPE, RUSSIA. "Auf der neuen Orenburgschen Linie, Lehmann".

Distribution in MA \& K: Kazakhstan (W: W Kazakhstan prov., Aktobe prov.; N: Kostonai prov., Akmola prov., N Kazakhstan prov., Pavlodar prov.; NE: E Kazakhstan prov.; Central: Karagandy prov.).

Distribution in other Asian countries: China.

Distribution area: Asia (Northern and Central), Europa.

Sium latifolium L., 1753, Sp. P1.: 251.

$\equiv$ Cicuta latifolia (L.) Crantz, 1767, Cl. Umb. Emend.: 97.

$\equiv$ Coriandrum latifolium (L.) Crantz, 1767, Stirp.

Austr. Fasc. 3: 101.

$\equiv$ Sium lancifolium Schrank, 1789, Baier. Fl.: 556.

$\equiv$ Pimpinella latifolia (L.) Stokes, 1812, Bot. Mat. Med. 2: 148.

$\equiv$ Drepanophyllum palustre Hoffm., 1814, Gen.

P1. Umbell.: 110, nom. illeg. (Art. 52.1).

$\equiv$ Sisarum palustre Bubani, 1900, Fl. Pyren. (Bubani) 2: 357, nom. illeg. (Art. 52.1).

$\equiv$ Selinum sium E. H. L. Krause, 1904, in Deutschl. Fl. (Sturm), ed. 2 12: 31, tab. 13.

$\equiv$ Drepanophyllum latifolium (L.) Koso-Pol., 1913, Trudy Bot. Sada Imp. Jur'evsk. Univ. 14: 141.

Type: EUROPE. "In Europae rivulos et ad ripas paludosas, Herb. Clifford 98, Sium 1" (lectotype BM-Cliff [BM000558303], designated by Reduron \& Jarvis in Jarvis et al., Regnus Veg. 127: 88 (1993)).

= Sisarum macrophyllum Schur, 1866, Enum. Pl. Transsilv.: 248.

Distribution in MA \& K: Kazakhstan (W: W Kazakhstan prov., Aktobe prov.; N: Kostonai prov., N Kazakhstan prov., Akmola prov., Pavlodar prov.; NE: E Kazakhstan prov.; Central: Karagandy prov.; S: Zhambyl prov.; SE: Almaty prov.).

Distribution in other Asian countries: Russia, Azerbaidzhan, Georgia.

Distribution area: Asia (Northern and Central), Europa, Australia \& Oceania (adv.).

Sium medium Fisch. et C. A. Mey., 1843, Index Sem. Horti Petrop. 9, Suppl.: 19.

$\equiv$ Drepanophyllum medium (Fisch. et C. A. Mey.) Koso-Pol., 1914, Trudy Tiflissk. Bot. Sada 16: 181.

Type: KAZAKHSTAN. "In humidis regionum altaicerum atque in desertis songoro-kirgisicis, VII 1826, Ledebour \& Meyer 269" (holotype LE! [LE01065719]).

$=$ Sium latijugum C. B. Clarke, 1879, in Fl. Brit. India (J. D. Hooker) 2: 683.
Type: INDIA. "Kashmir, alt. 5000-7000 ft., 1847. Thomson 300" (lectotype K! [K000685623]: designated by Pimenov, 2017, in Turczaninowia 20, 2: 222; isolectotypes $\mathrm{K}$ [K000685622], MANCH!, $\mathrm{P} !)$.

Distribution in MA \& K: Kazakhstan (W: Aktobe prov.; N: Akmola prov.; NE: E Kazakhstan prov.; Central: Karagandy prov.; S: S Kazakhstan prov., Zhambyl prov.; SE: Almaty prov.), Kyrgyzstan (Batken prov., Jalal-Abad prov., Ysyk-köl prov., Naryn prov., Osh prov., Talas prov., Chüh prov.), Tajikistan (Sughd prov., Kŭhistani Badakhshan, Khatlon prov., Dushanbe prov.), Uzbekistan (Qashqadarya prov., Namangan prov., Toshkent prov.).

Distribution in other Asian countries: China, India, Pakistan, Afghanistan.

Distribution area: Asia (Central, SouthWestern, Eastern)

Sium sisaroideum DC., 1830, Prodr. (A. P. de Candolle) 4: 124.

三Sisarum sisaroideum (DC.) Schischk., 1935, in

Krylov, Fl. Zapadnoi Sibiri 8: 2077.

E Pimpinella sisaroidea (DC.) Manden., 1950, in

Fl. Georgiae 2 $2^{\text {nd }}$ ed, 6: 425.

$\equiv$ Sium sisarum L. subsp. sisaroideum (DC.) Soó, 1978 [1977] Acta Bot. Acad. Sci. Hung. 23(34): 380 .

Type: IRAN. "In arenosis umbrosis circa Badalan, Distr. Khoi, Prov. Aderbeizan, Persiae, 20 VII 1828 ,

Szovits 561" (lectotype G-DC!; designated by Pimenov, 2017, in Turczaninowia 20, 2: 222; isolectotype LE!).

= Sium lancifolium M. Bieb., 1819, Fl. Taur.Cauc. 3: 230, nom. illeg. (Art. 53.1), non Schrank (1789).

= Berula lancifolia Besser, 1822, Enum. pl. (Besser): 44.

=Pimpinella sisarum (L.) Koso-Pol. var. lancifolia (Besser) Koso-Pol., 1914, Bull. Soc. Imp. Naturalistes Moscou, n. s. 28: 180.

= Sium sisarum L. var. lancifolium (Besser) Thell., 1926, in Ill. Fl. Mitt.-Eur. 5(2): 1233.

Type: UKRAINE. "In paludosis inter virgulta salicina et Opulum. per Rossiam australioram, usque Ucrainiam [Tanais. 1816. Henning" (LE-Bieb!).

= Sium lancifolium M. Bieb. var. elongatum Parsa, 1948, Kew Bull. 1948: 200.

Type: IRAN. "N Persia, hills above Gilas, 657500 ft. 29 V 1929. Parsa" (Herb. Parsa). 
- Sium sisarum auct. non L.: Ledeb., 1829, F1. Altaic. (Ledebour) 1: 351.

- Sium latifolium auct. non L.: Wang Tiehteng, 1985, in Fl. Reipubl. Popularis Sin. 55(2): 157, tab. 65, fig. 7-9, p. p.

Distribution in MA \& K: Kazakhstan (W: W Kazakhstan prov., Aktobe prov.; N: Kostonai prov., Akmola prov., N Kazakhstan prov., Pavlodar prov.; NE: E Kazakhstan prov.; Central: v., Karagandy prov.; S: S Kazakhstan prov., Zhambyl prov.; SE: Almaty prov.), Kyrgyzstan (Jalal-Abad prov., Osh prov., Chüh prov.), Tajikistan (Sughd prov., Khatlon prov., Dushanbe prov.), Uzbekistan (Dshizak prov., Samarqand prov., Surxondarya prov., Toshkent prov., Fergona prov.), Turkmenistan (Balkan prov., Akhal prov.).

Distribution in other Asian countries: Russia, China, Afghanistan, Iran, Azerbaidzhan, Georgia, Armenia, Turkey, Iraq.

Distribution area: Asia (Northern, Central, South-Western and Mediterranean), Europa.

Smyrnium cordifolium Boiss., 1846, Diagn. P1. Orient. ser. 1, 6: 64.

Type: IRAN. "In faucibus saxosis umbrosis montium prope Persepolin Persiae australis. [In faucibus saxosis umbrosis prope ruinas u. Persepolis], 20 IV 1842, Kotschy 803" (lectotype G-BOIS!; designated as "holotypus" by Rechinger (1987: 163); isolectotypes BM! [BM000944700, [BM000944701], G! [G00370263, G00370264], K! [K000697410], LE! [LE00015693, LE00015694], MO [MO-3456505], P [P00834440-P00834442], US [US0127079]).

= Smyrnium androssovii Korovin, 1950, in Fl. Turkmen. 5: 192. nom. inval., descr. ross. (Art 39.1)

Distribution in MA \& K: Turkmenistan (Mary prov.).

Distribution in other Asian countries: Afghanistan, Iran, Turkey, Iraq, Syria.

Distribution area: South-Western Asia.

Sphaenolobium korovinii Pimenov et Kljuykov, 2014, Bot. Zhurn. (St. Petersburg) 99(5): 602, fig. 4.

Type: KAZAKHSTAN. "Kazachstania australis, montes Karatau Syrdariensis, jugum Boroldai, vallis sicca Bastargai. 10 VIII 1982, Pimenov, Vasilieva \& S. Daushkevich 70" (holotype MW! [MW0595553]).

- Selinum coriaceum Korovin, 1924, Bot. Mater. Gerb. Glavn. Bot. Sada R.S.F.S.R. 5(6): 77, quoad descr., excl. typus.

Distribution in MA \& K: Kazakhstan (S: S. Kazakhstan prov.). Endemic.
Distribution area: South-Western Asia.

Sphaenolobium tenuisectum (Korovin) Pimenov, 1975, Novit. Syst. Pl. Vasc. 12: 244.

三Selinum tenuisectum Korovin, 1947, Not. Syst. Herb. Inst. Bot. Zool. Acad. Sci. Uzbekistan 8: 14.

Type: UZBEKISTAN. "Tian-Schan occidentalis, in valle fl. Pskent, ad locum Anar, 31 VII 1936, Dmitrieva" (holotype AA).

Distribution in MA \& K: Uzbekistan (Toshkent prov.). Endemic.

Distribution area: South-Western Asia.

Sphaenolobium thianschanicum (Korovin) Pimenov, 1975, Novit. Syst. Pl. Vasc. 12: 243.

三Selinum thianschanicum Korovin 1924, Bot. Mater. Gerb. Glavn. Bot. Sada R.S.F.S.R. 5(6): 76.

Type: UZBEKISTAN. "Mont. Thian-Schan occidentalis, in valle Ugam, Chordshuman-sai prope pag. Chumsan, 06 VIII 1920, Popov 1068, 1069, 1070, 1071, 1072, 1073" (lectotype TASH! [TASH002345]; designated here by Pimenov; isolectotypes LE! [LE01065762, LE01065763], MHA!, TASH! [TASH002346-TASH002348]).

= Selinum coriaceum Korovin, 1924, Bot. Mater. Gerb. Glavn. Bot. Sada R.S.F.S.R. 5(6): 77, quoad typus.

= Sphaenolobium coriaceum (Korovin) Pimenov, 1983, in Vvedensky (ed.), Opred. Rast. Sred. Azii 7: 264, p. p.

Type: UZBEKISTAN. "In valle fluv. Angren, ad locos lapidosos in partibus superioribus flum. Kamtschik-sai, 08 VI 1924. Korovin 580" (lectotype TASH! [TASH002343]; designated here by Pimenov; isolectotype MHA!).

= Selinum tianschanicum Korovin var. transitorium Korovin, 1926, Byull. Sredne-Asiatsk. Gosud. Univ. 14, Suppl. (Sched. Herb. Fl. As. Med. 10): N 242.

Type: UZBEKISTAN. "Prov. Syr-Darja, distr. Taschkent, ad declivia saxosa in regione alpina montis Tschimgan Majoris, 29 VII 1925, Korovin 242" (lectotype TASH!; designated here by Pimenov; isolectotypes C! [C10008578], MHA!, MW! [MW05939329], MW0593929], W! [W 1927 0011686]).

Distribution in MA \& K: Kazakhstan (S: S Kazakhstan prov., Zhambyl prov.), Kyrgyzstan (Jalal-Abad prov.), Tajikistan (Sughd prov.), Uzbekistan (Toshkent prov.). Endemic.

Distribution area: South-Western Asia. 
Sphaerosciadium denaense (Schischk.)

Pimenov et Kljuykov, 1981, Bot. Zhurn. (Leningrad) 66(4): 480 .

$\equiv$ Danaa denaensis Schischk., 1950, in Schischkin (ed.), Fl. URSS 16: 593, 225, tab. 15, fig. 8.

$\equiv$ Physospermum denaense (Schischk.) B. Fedtsch. ex Weinert, 1970, Hercynia 7(1-3): 125.

$\equiv$ Astomatopsis denaensis Korovin ex Kamelin, 1973, Florog. Anal. Fl. Sred. Azii: 94, nom. illeg. sine basion. compl.

Type: UZBEKISTAN. "Buchara, Denau, jugum Hissaricum, in faucibus fl. Sangardak. 22 V - 04 VI 1913. Michelson 1742" (holotype LE! [LE00051268], isotypes LE! [LE00051269, LE00051270]).

Distribution in MA \& $\mathbf{K}$ : Uzbekistan (Surxondarya prov.). Endemic.

Distribution area: South-Western Asia.

Stenocoelium athamantoides (M. Bieb.) Ledeb., 1829, Fl. Altaic. (Ledebour) 1: 298.

$=$ Cachrys athamantoides M. Bieb. 1819, Fl.

Taur.-Cauc. 3: 217, sine typo indicato.

$=$ Rumia athamantoides (M. Bieb.) DC., 1830, Prodr. (A. P. de Candolle) 4: 98.

Type: RUSSIA. "Altai, in lapidosis alpinis ad fl. Tschuja contra ostium fl. Tschagan locis lapidosis. [In alpibus contra ostium fluvii Tschagan locis lapidosis], VII 1826, Bunge 227" (neotype LE!; designated by V. M. Vinogradova (2005: 966); isoneotype LE!).

Distribution in MA \& K: Kazakhstan (NE: E Kazakhstan prov.).

Distribution in other Asian countries: Russia.

Distribution area: Northern and Central Asia.

Stenocoelium trichocarpum Schrenk, 1841, Enum. P1. Nov. 1: 80; id., 1843, Bull. Phys.-Math. Acad. Petersb. 1: 80.

$\equiv$ Seseli trichocarpum (Schrenk) B. Fedtsch., 1915, Rastit. Turkest.: 616.

Type: KAZAKHSTAN. "Songoria, in monte Tarbagatai, 25 VIII 1841, Schrenk 963" (neotype LE! [LE01066056]; designated by Pimenov, 2017, in Turczaninowia 20, 2: 225; isoneotypes AA!, LE! [LE01066057-LE01066061], MANCH!, P!).

Distribution in MA \& K: Kazakhstan (NE: E Kazakhstan prov.).

Distribution in other Asian countries: China.

Distribution area: Central Asia.

Tetrataenium olgae (Regel et Schmalh.) Manden. 1959, Trudy Tbilis. Bot. Inst. 20: 8. $\equiv$ Heracleum olgae Regel et Schmalh., 1882, Izv. Obshch. Ljubit. Estestv. 34(2) (Descr. P1. Nov. Rar. Fedtsch.): 38 .

$\equiv$ Pastinaca olgae (Regel et Schmalh.) KosoPol., 1916, Bull. Soc. Imp. Naturalistes Moscou, n. s. 29: 113, nom. inval., non Regel et Schmalh. (1882).

$\equiv$ Platytaenia olgae (Regel et Schmalh.) Korovin, 1959, in Vvedensky (ed.), Fl. Uzbekist. 4: 465.

Type: KYRGYZSTAN. "In Kokaniae trajectus Isfairam. [In descendi trajectus Isfairam in Alai], 8000 ' alt., 20 VII 1871, O. A. Fedtschenko" (lectotype LE! [LE00052206], designated by V. M. Vinogradova 2001: 51; isolectotypes LE [LE00052207, LE00052208]); TAJIKISTAN. "In valle fl. Sarawschan inter Obburdon et Pachud, alt. 5-6000', O. A. Fedtschenko" (syntype LE!); "In montibus Naubod prope Varsaminor, alt. $4500-8000$ ', O. A. Fedtschenko" (syntype LE!).

= Heracleum polyadenum Rech. f., 1963, in Biol. Skg. 13, 4 (Symb. Afghan. 5): 85.

Type: AFGHANISTAN. "Munjan: Sanglich, 3450 m, 24 VII 1937, Koelz 12617" (holotype US! [US00289210, US0028911]; W! [W0053820 photo]).

- Heracleum nubigenum auct. non C. B. Clarke: M. Hiroe, 1958, Umbellif. Asia 1: 191.

Distribution in MA \& K: Kyrgyzstan (Batken prov., Jalal-Abad prov., Naryn prov., Osh prov.), Tajikistan (Kŭhistani Badakhshan, Sughd prov., Khatlon prov., Dushanbe prov.), Uzbekistan (Dzhizak prov.).

Distribution in other Asian countries: China, Pakistan, Afghanistan.

Distribution area: South-Western Asia.

Tordylium maximum L., 1753, Sp. Pl.: 240.

$\equiv$ Caucalis maxima (L.) Vest, 1805, Man. Bot. (Vest.): 492.

$\equiv$ Heracleum tordylium Spreng., 1813, Neue Schr. Naturf. Ges. Halle 2, 1 (P1. Umb. Prodr.): 12.

$\equiv$ Selinum tordyliastrum E. H. L. Krause, 1904, in Fl. Deutschl. (Sturm) ed. 2 12: 140, nom. illeg. (Art. 52.1).

$\equiv$ Pastinaca tordylium (Spreng.) Calest. 1905, Webbia 1: 247., nom. illeg. (Art. 52.1).

$\equiv$ Pastinaca maxima (L.) Koso-Pol., 1922, Schedae Herb. Fl. Ross. 8: 115.

Type: EUROPE, ITALY. "In Italiae ruderatis sepibus [Cultivated material from the garden of Georg Clifford], Herb. Clifford 90, Tordylium 4 "(lectotype BM-Cliff [BM000558244], designated by Al-Eisawi \& Jury (1988: 390) and in Jarvis et 
al., 1993: 94); Leiden, van Royen, syntype L! [L0053028].

$=$ Dumaniana gelendostensis Y1ld. et B. Selvi, 2006, Ot Sist. Bot. Dergisi 13(2): 5, fig. 1, 2, map 3.

$=$ Pastinaca gelendostensis (Y1ld. et B. Selvi) Hand, 2011, Willdenowia 41(2): 249.

Type: TURKEY. "Turkey. B3 Isparta: Gelendost, Akdağ Köyü, Kirişli or Çatalgöl daği, from SE part of mountain to Çatalgöl, association of Quercus coccifera, 1000-1500 m, 12 VI 2002, Selvi 2420" (holotype Hb. Yildırımlı; isotype GAZI).

Distribution in MA \& K: Turkmenistan (Balkan prov.).

Distribution in other Asian countries: Russia, Iran, Azerbaidzhan, Georgia, Armania, Turkey, Cyprus.

Distribution area: Asia (Northern, SouthWestern and Mediterranean), Europa.

Torilis arvensis (Huds.) Link, 1821, Enum. Hort. Berol. Alt. 1: 265.

$\equiv$ Caucalis arvensis Huds, 1762, Fl. Angl.: 98.

$\equiv$ Torilis divaricata Moench, 1802, Methodus (Moench) Suppl.: 84, nom. illeg. (Art. 53.1), non Wibel (1790).

$\equiv$ Anthriscus arvensis (Huds.) Koso-Pol., 1916, Bull. Soc. Imp. Naturalistes Moscou, n. s. 29: 151.

$\equiv$ Torilis arvensis (Huds.) Link subsp. divaricata Thell. 1926, in Hegi, Ill. Fl. Mitt. - Eur. 5, 2: 1055.

Type: EUROPE. "Britania [Surrey Vice County 17 Box Hill chalky field], 03 IX 1920. Fogitt" (neotype BM, designated by Jury et al. in Reduron 2008: 2506).

= Scandix infesta L., 1767, Syst. Nat., ed. 12, 2: 732.

$=$ Torilis infesta (L.) Clairville, 1811, Man. Herboris. Suisse et Valais: 78.

Type: EUROPE. "Sweden, Uppsala, 364.10" (lectotype LINN [LINN-HL364-10], designated by Townsend in Polhill (ed.), 1989: 27).

$=$ Caucalis angustifolia Forssk. 1775, Fl. Aegipto-Arab.: 206.

Type: YEMEN. "Al Hadiyh ("Hadie"). III 1763, Forsskål 668” (holotype C! [C10001846]).

$=$ Caucalis helvetica Jacq. 1776, Hort. Vindob. 3: 12, tab. 16.

$=$ Torilis helvetica (Jacq.) C. C. Gmelin, 1805, Fl. Bad. 1: 617.

Type: "Plants grown in Vienna bot. gard. from the seeds collected in Switzerland (ex Helvetia), 364/10?" (holotype LINN).

$=$ Caucalis infesta Curt. 1821, Flora Londinensis 2(6): tab. 23.
$=$ Lappularia infesta (Curt.) Pomel, 1874, Nouv. Matér. Fl. Atl.: 150.

$=$ Torilis helvetica (Jacq.) C. C. Gmelin var. pauciradiata Trautv. 1871, Trudy Imp. S.-Peterburgsk. Bot. Sada 1: 33 .

Distribution in MA \& K: Kazakhstan (S: S Kazakhstan prov., Zhambyl prov.), Kyrgyzstan (Batken prov., Jalal-Abad prov., Osh prov.), Tajikistan (Kŭhistani Badakhshan, Sughd prov., Khatlon prov., Dushanbe prov.), Uzbekistan (Navoi prov., Dzhizak prov., Samarqand prov., Qashqadarya prov., Surxondarya prov., Sirdarya prov., Toshkent prov., Fergona prov.), Turkmenistan (Balkan prov., Akhal prov., Lebap prov., Mary prov.).

Distribution in other Asian countries: Russia, India, Pakistan, Afghanistan, Iran, Azerbaidzhan, Georgia, Armania, Turkey, Itraq, Saudi Arabia, Yemen, Lebanon, Syria, Israel, Jordan, Egypt (Sinai), Cyprus.

Distribution area: Asia (Northern, SouthWestern, Mediterranean and W Tropical), Europa, Africa, N. America (adv.), C. America (adv.), Australia \& Oceania (adv.).

Torilis arvensis (Huds.) Link subsp. turcomanica Geld. 1985, Bot. Zhurn. (Leningrad) 70(12): 1645.

Type: TURKMENISTAN. "Turcomania, Kopetdag occidentalis, distr. Karakala, in angustiis Iol-dere, ad fundum angustiarum, 13 VI 1983, Geldychanov" (holotype LE [LE00051028]).

Distribution in Asia: Turkmenistan (Balkan prov.).

Distribution area: Endemic.

Torilis leptophylla (L.) Rchb.f. 1867, in Icon. Fl. Germ. Helv. (Reichenbach) 21: 83, tab. 169.

$\equiv$ Caucalis leptophylla L., 1753, Sp. P1.: 242.

$\equiv$ Daucus leptophyllus (L.) Scop. 1771, Fl. Carniol. (ed. 2) 1: 190.

$\equiv$ Pullipes leptophylla (L.) Raf. 1840, Good Book: 54.

$\equiv$ Daucalis leptophylla (L.) Pomel, 1874, Nouv. Matér. Fl. Atl.: 149.

$\equiv$ Nigera parviflora Bubani, 1900, Fl. Pyren. (Bubani) 2: 405, nom. illeg. (Art. 52.1).

$\equiv$ Anthriscus leptophylla (L.) Koso-Pol., 1916, Bull. Soc. Imp. Naturalistes Moscou, n. s. 29: 151.

Type: EUROPE. "In Gallia, Italia 338.1 3" (neotype LINN, designated by Reduron 2008: 2533).

= Caucalis humilis Jacq. 1772, Hort. Vindob. 2: 92. 
$=$ Caucalis parviflora Lam., 1783, in Encycl. (Lamarck et al.) 1(2): 657.

Type: EUROPE, FRANCE. "Dans les champs de Provinces meridionales de la France".

$=$ Caucalis xanthotricha Steven, 1856, Bull. Soc. Imp. Naturalistes Moscou 29(2): 353.

$=$ Torilis xanthotricha (Steven) Stankov, 1949, in Stankov et Taliev, Sist. Class. Rast. Evrop. Rossii 533.

Type: EUROPE, CRIMEA. "Laspi. [In Tauria], Compere" (lectotype LE!; designated here; isolectotype $\mathrm{H}$ [H1132433]).

$=$ Caucalis leptophylla L. var. peduncularis Boiss. et Noë, 1856, Diagn. Pl. Orient. (ser. 2) 2: 104.

Type: IRAQ. "In Kurdistanâ, VI 1849, Noё 203" (holotype G-BOIS!, see: Pimenov \& Jacquemoud (2020: 83)).

= Caucalis gaillardotii Boiss., 1972, Fl. Orient. (Boissier) 2: 1085.

$=$ Torilis gaillardotii (Boiss.) Drude, 1898, in Nat. Pflanzenfam. (Engler et Prantl) 3(8): 156.

Type: SYRIA. "In lapidosis ad radices montis Gebel Khaisoon prope Damascum [calc. pierreux au pied du Gebel Khaisoon, 4000'], 15 VIII 1856, Gaillardot 1817" (lectotype G-BOIS!; designated by Pimenov \& Jacquemoud (2020: 83); isolectotype $\mathrm{P}$ [P00834464]).

$=$ Caucalis tenuisecta Pau,1918, Trab. Mus. Nac. Ci. Nat., Ser. Bot. 14: 25.

Type: IRAN. "[Persia]. Gotvend (bajo Karum), 400 m, 9-20 IV 1899, Martinez de la Escalera" (syntypes E (photo), MA); "Valle de Bazouft [Gotvend], 2000 m, 08 VII 1899, Martinez de la Escalera" (syntypes E (photo), MA).

- Caucalis pumila auct. non L.: Lam., 1779, Fl. franç. 3: 425.

Distribution in MA \& K: Kazakhstan (S: S Kazakhstan prov.), Kyrgyzstan (Batken prov., JalalAbad prov., Osh prov., Chüh prov.), Tajikistan (Kŭhistani Badakhshan, Sughd prov., Khatlon prov., Dushanbe prov.), Uzbekistan (Dzhizak prov., Qashqadarya prov., Surxondarya prov., Toshkent prov., Andijon prov.), Turkmenistan (Balkan prov., Akhal prov., Lebap prov., Mary prov.).

Distribution in other Asian countries: Russia, Japan, India, Pakistan, Afghanistan, Iran, Azerbaidzhan, Georgia, Armenia, Turkey, Iraq, Saudi Arabia, Lebanon, Syria, Israel, Jordan, Egypt (Sinai), Cyprus.

Distribution area: Asia (Northern, Eastern, South-Western, Mediterranean and W Tropical), Europa, Africa, N. America.
Torilis nodosa (L.) Gaertn. 1788, Fr. Sem. Pl. 1: 82 , tab. 20 , fig. 6 .

$\equiv$ Tordylium nodosum L.1753, Sp. P1.: 240.

$\equiv$ Caucalis nodosa (L.) Crantz, Cl. umbell. emend.: 112. 1767.

三 Caucalis nodiflora Lam., 1779, Fl. franç. 3: 424, nom. illeg (Art. 52.1).

$\equiv$ Tordylium nodiflorum Salisb., 1796, Prodr. stirp. Chap. Allerton: 160.

$\equiv$ Anthriscus nodosa (L.) Pers. 1805, Syn. Pl. 1: 320.

$\equiv$ Lappularia nodosa (L.) Pomel, 1874, Nouv. Matér. Fl. Atl.: 150.

$\equiv$ Torilis nodiflora Bubani, 1900, Fl. Pyren. (Bubani) 2: 406.

$\equiv$ Daucus nodosa (L.) E. H. L. Krause, 1904, in Deutschl. Fl. (Sturm) ed. 2 12: 137.

$\equiv$ Anthriscus nodiflora Koso-Pol., 1916, Bull. Soc. Imp. Naturalistes Moscou, n. s. 29: 151

Type: EUROPE. "Gallia, Italia ad vias, 337.6" (lectotype LINN [LINN-HL337-6], designated by Jafri in Jafri et El-Gadi, 1985: 116).

Distribution in MA \& K: Tajikistan (Khatlon prov., Dushanbe prov.), Uzbekistan (Qashqadarya prov., Surxondarya prov.), Turkmenistan (Balkan prov., Akhal prov.).

Distribution in other Asian countries: Russia, India, Pakistan, Afghanistan, Iran, Azerbaidzhan, Georgia, Turkey, Iraq, Saudi Arabia, Oman, Lebanon, Syria, Israel, Jordan, Cyprus.

Distribution area: Asia (Northern, Easytern, South-Western, Mediterranean and W Tropical), Europa, Africa, N. America (adv.), S. America (adv.), Australia \& Oceania (adv.).

Torilis stocksiana (Boiss.) Drude, 1898, in Nat. Pflanzenfam. (Engler et Prantl) 3(8): 156.

$\equiv$ Caucalis stocksiana Boiss., 1859, Diagn. Pl. Orient. (ser. 2) 6: 89.

$\equiv$ Anthriscus stocksiana (Boiss.) Koso-Pol., 1916, Bull. Soc. Imp. Naturalistes Moscou, n. s. 29: 151. R.S.F.S.R. 36(1) (Fl. As. Ross. 15): 97.

Type: PAKISTAN. "In ditione Beloutschistan circa Khozdar [Ad urbem Khozdar], 1850, Stocks 775" (lectotype G-BOIS! [G00150265]; designated as "typus" by Peev (1987: 129); Beloutchistan, Stocks 1859 (isolectotypes G-BOIS! [G00150265, G00150266], K! [K000695535, K000695536]).

Distribution in Asia: Turkmenistan (Balkan prov., Akhal prov.).

Distribution in other Asian countries: Russia, India, Pakistan, Afghanistan, Iran, Azerbaidzhan, Iraq, Oman, UAE. 
Distribution area: Eastern, South-Western and W Tropical Asia.

Trachyspermum ammi (L.) Sprague ex Turrill, 1929, Bull. Misc. Inform. Kew (1929): 228.

三Sison ammi L., 1753, Sp. P1.: 252.

三Aethusa ammi (L.) Spreng., 1814, Mag. Neuersten Entdesk. Gesammten Naturk. Ges. Naturf. Freude Berlin 6: 260.

$\equiv$ Carum ammi (L.) Sprague, 1922, J. Bot. 60: 314 , in obs.

Type: "In Apulia, Aegypto, 356.5" (lectotype LINN [LINN-HL365-5], designated by Jansen (1981: 114)).

=Ammi copticum L., 1767, Mant. P1. 1: 56.

= Daucus copticus (L.) Lam., 1785, in Encycl. (Lamarck et al.) 1(2): 635.

= Bunium copticum (L.) Spreng., 1813, Neue Schr. Naturf. Ges. Halle 2(1) (Pl. Umb. Prodr.): 28.

= Trachyspermum copticum (L.) Link, 1821, Enum. Hort. Berol. Alt. 1: 267.

= Ptychotis coptica (L.) DC., 1829, Mém. Soc. Phys. Genève 4: 496.

= Carum copticum (L.) Benth. ex Hiern, 1877, in

F1. Trop. Afr. (Oliver et al.), 3: 12.

= Selinum copticum (L.) E. H. L. Krause, 1904, in Deutschl. Fl. (Sturm), ed. 2 12: 43.

Type: AFRICA, EGYPT. "In Aegypto, Forsskål" [cultivated in Uppsala]" (lectotype LINN [LINNHL341-4]; designated by Townsend in Polhill, 1989: 45).

= Bunium aromaticum L., 1771, Mant. P1. Alt.: 218.

Type: GREECE/SYRIA. "In Creta, Syria, VIII: 43" (lectotype UPS-Burser VIII: 43, designated by Watson in Jarvis et al. 2006: 209).

= Ligusticum ajowain Roxb. in Fleming, 1810, Asiat. Res. 11: 171.

= Ptychotis ajowain (Roxb.) DC., 1829, Mém. Soc. Phys. Genève 4: 497.

= Carum ajowain (Roxb.) Benth. 1867, in Gen.

P1. (Bentham et Hooker f.), 1(3): 891.

Type: INDIA. Roxburgh [572] (lectotype $\mathrm{K}$ !; designated here by Pimenov; isolectotype $\mathrm{K}-\mathrm{WALLICH}$ !).

= Ammi glaucifolium Blanco, 1837, Fl. Filip.:

213, nom. illeg. (Art. 53.1), non L. (1753).

= Daucus anisodorus Blanco, 1845, F1. Filip. (ed. 2): 150 .

Type: PHILIPPINES. Cultivated plants.

= Deverra korolkowii Regel et Schmalh., 1878, Trudy Imp. S.-Peterburgsk. Bot. Sada 5(2): 589, "korolkowi".
= Pituranthos korolkowii (Regel et Schmalh.) Schinz, 1894, Bull. Herb. Boissier 2: 211, "korolkowi".

= Carum korolkowii (Regel et Schmalh.) Lipsky, 1900, Trudy Imp. S.-Peterburgsk. Bot. Sada 18(1): 66.

Type: UZBEKISTAN. "In hortis urbis Chiva cultum sub nomine Mumuk [Chiva], 1873 Korolkoff \& Krause" (holotype LE! [LE00051468], isotype LE! [LE00051469]).

Distribution in MA \& K: Uzbekistan (Andijon prov., Buxoro prov., Namangan prov., Samarqand prov., Surxondarya prov., Toshkent prov., Fergona prov., Khorezm prov.), Turkmenistan (Daşoguz prov., Lebap prov.).

Distribution in other Asian countries: Philippines, Indonesia, Myanmar, Bangladesh, Bhutan, Nepal, India, Pakistan, Afghanistan, Iran, Oman, Saudi Arabia, Israel.

Distribution area: Asia (Eastern, SouthWestern, W Tropical, and E Tropical), Africa.

Trinia hispida Hoffm., 1814, Gen. P1. Umbell.: 94.

三 Trinia hoffmannii M. Bieb. 1819, Fl. Taur.Cauc. 3: 244, nom. illeg. (Art. 52.1).

三 Apinella hoffmannii Calest. 1905, Webbia 1: 145, nom. illeg. (Art. 52.1).

$\equiv$ Rumia hispida (Hoffm.) Stankov, 1949, in Stankov et Taliev, Syst. Classif. Vasc. P1. Eur. Russ.: 526.

Type: RUSSIA (EUR.). "In siccis desertis Rossiae, ad coloniam Sareptam [Sarepta], Marchall von Bieberstein" (lectotype LE!; designated here by Pimenov; isolectotypes BM, LECB [LECB0000086], MW).

= Rumia leiogona C. A. Mey. 1831, Verz. Pfl. Casp. Meer. (C. A. von Meyer): 125.

$=$ Trinia leiogona (C. A. Mey.) B. Fedtsch., 1915, Rastit. Turkest.: 608.

$=$ Trinia hispida Hoffm. subsp. leiogona (C. A. Mey.) Fedor.1978, Bot. Zhurn. (Leningrad) 63(3): 613.

Type: AZERBAIDZHAN. "In montibus altioribus Talusch (alt. 800-1000 hexap.), Meyer" (syntype LE); ARMENIA. "In campis subalpinis, 17 VII 1830, Meyer" (lectotype LE!; designated by M. M. Fedoronchuk 1979: 49).

= Trinia scabra Boiss. et Noë, 1856, Diagn. P1. Orient. (ser. 2) 2: 73.

Type: TURKEY. "In montanis circâ Sivas Anatoliae, VI 1852, Noё 882" (lectotype G-BOIS!; designated here by Pimenov et Jacquemoud; 
isolectotype BM! [BM000944710]; "Plain située entre le Zamanta-sou et l'Aslan-Dagh (Cappadoce). 07 VIII 1856. Balansa" (epitype P [P032114083]).

- Pimpinella dioica M. Bieb. 1808, Fl. Taur.Cauc. 1: 242, nom. illeg. (Art. 53.1), non L. (1774).

Type: EUROPE, "Tauria (type ?)" BM [BM000752007]).

= Pimpinella humilis Willd. ex Ledeb., 1844, Fl. Ross. (Ledebour) 2: 281, nom. inval. (Art. 36.1 \& 39.1).

- Trinia hoffmannii auct., non M. Bieb.: Boiss., 1872, Fl. Orient. (Boissier) 2: 833.

Distribution in MA \& K: Kazakhstan (W: W Kazakhstan prov., Aktobe prov.; N: Kostonai prov.).

Distribution in other Asian countries: Russia, Iran, Azerbaidzhan, Georgia, Armenia, Turkey.

Distribution area: Asia (Northern and Central), Europa.

Trinia muricata Godet, 1852, Fl. Jura 1: 271, in adnota.

$\equiv$ Trinia ramosissima Ledeb. subsp. muricata (Godet) Fedor. 1978, Bot. Zhurn. (Leningrad) 63(3): 613.

Type: EUROPE. Jura, Godet".

$=$ Trinia lessingii Rchb. f. 1867, in Icon. Fl. Germ. Helv. (Reichenbach) 21: 8 .

Type: RUSSIA. "Rossia, Lessing".

Distribution in Asia: Kazakhstan (W: Aktobe prov.; N: Kostonai prov., Akmola prov.; NE: E Kazakhstan prov.; Central: Karagandy prov.).

Distribution in other Asian countries: Russia.

Distribution area: Asia (Northern and Central), Europa.

Trinia ramosissima Ledeb., 1829, Fl. Altaic. (Ledebour) 1: 357.

$\equiv$ Trinia polyclada Schischk., 1950, in Schischkin (ed.), Fl. URSS 16: 351.

Type: KAZAKHSTAN. "In planitiebus ad fl. Irtysch, prope pag. Krasnojarsk et alibi, Ledebour" (syntype LE); "Circa fortalitium Buchtarminsk [In campestribus siccis prope Buchtarminsk versus Arkat], Meyer" (lectotype LE! [LE00051456], designated by M. M. Fedoronchuk 1983: 48).

- Trinia kitaibelii auct. non M. Bieb.: O. et B. Fedtsch., 1909, Consp. Fl. Turkest. 3: 79.

- Rumia leiogona auct. non C. A. Mey.: O. et B. Fedtsch., 1909, Consp. Fl. Turkest. 3: 96.

Distribution in MA \& $\mathrm{K}$ : Kazakhstan $(\mathrm{N}$ : Kostonai prov., Pavlodar prov.; NE: E Kazakhstan prov.; Central: Karagandy prov. SE: Almaty prov.).

Distribution in other Asian countries: Russia.
Distribution area: Norhtern and Central Asia.

Note: The correct name of this SiberianKazakhstanian species is not completely clear. The name of Trinia ramosissima Ledeb. can be accepted only if $T$. ramosissima (Fisch. ex Trev.) W. D. J. Koch would be recognized as nomen invalidum. Its basionym, Pimpinella ramosissima Fisch. ex Trev. is nomenclaturally invalid, but this qualification does not mean that Trinia ramosissima W. D. J. Koch is also not a valid name. Cannon (1968: 351) used the name of T. ramosissima (Fisch. ex Trev.) W. D. J. Koch for another, European species of Trinia, here treated, following Fedoronchuk (1983), as T. kitaibelii M. Bieb.

Tschulaktavia saxatilis (Bajtenov) Bajtenov ex Pimenov et Kljuykov, 2012, Bot. Zhurn. (St. Perersburg) 97(5): 652.

ESilaus saxatilis Bajtenov, 1979, Bot. Mater. Gerb. Inst. Bot. Akad. Nauk Kazakh. SSR 11: 58.

Type: KAZAKHSTAN. "Tian-Schan borealis, montes Tschulaktau, loco Taldy-sai, in fissuris rupium. 20 V 1976. Bajtenov" (neotype AA!; designated by Pimenov et Kljuykov 2012: 76; isoneotype MW! [MW0861203]); “ibid. 10 VI 1956. Goloskokov" (syntype LE [LE01066092]).

Distribution in Asia: Kazakhstan (SE: Almaty prov.). Endemic.

Distribution area: South-Western Asia.

Turgenia latifolia (L.) Hoffm., 1814, Gen. Pl. Umbell.: 59.

$\equiv$ Tordylium latifolium L., 1753, Sp. Pl.: 240.

$\equiv$ Caucalis latifolia (L.) L., 1767, Syst. Nat. (ed. 12) 2: 205 .

$\equiv$ Daucus latifolius (L.) Baill., 1879, Hist. P1. (Baillon) 7: 193, "latifolia".

$\equiv$ Daucus turgenia E. H. L. Krause, 1904, in Deutschl. Fl. (Sturm) ed. 2 12: 158.

Type: EUROPE. "Gallia et Italia 338.7" (lectotype LINN! [LINN-HL338-7], designated by Reduron et Jarvis in Jarvis et al. 2006: 210; see also Reduron, 2008: 2580).

$=$ Turgenia multiflora DC., 1830, Prodr. (A. P. de Candolle) 4: 218.

$=$ Turgenia latifolia (L.) Hoffm. var. multiflora (DC.) Boiss., 1872, Fl. Orient. (Boissier) 2: 1087.

Type: TURKEY. "In agro Bysantino, Prescott" (holotype G-DC!).

= Turgenia brachyacantha Boiss., 1844, Ann. Sci. Nat. sér. 3, 2: 52.

$=$ Turgenia latifolia (L.) Hoffm. var. brachyacantha (Boiss.) Boiss., 1872, Fl. Orient. (Boissier) 2: 1087. 
Type: IRAN. "In Persia australi, Aucher-Eloy [4563,4564] (lectotype P! [P03260926], designated by Pimenov, 2017, in Turczaninowia 20, 2: 231); isolectotypes G-BOIS!, P [P02284847, P03260927, P03260931, P03260961, P03260978]).

= Turgenia tuberculata Boiss., 1844, Ann. Sci. Nat. sér. 3, 2: 52.

$=$ Turgenia latifolia (L.) Hoffm. var. tuberculata (Boiss.) Boiss., 1872, Fl. Orient. (Boissier) 2: 1087.

Type: SYRIA. "In Syriâ, Aucher-Eloy 3639" (lectotype G-BOIS!: designated by Pimenov \& Jacquemoud, 2017, in Turczaninowia 20, 2: 229); isolectotypes LE!, MPU! [MPU021041], P [P03260969]); "In agris prope Aleppum. $07 \mathrm{~V} 1841$. Kotschy 191" (syntypes G-BOIS!, P [P02284849, P03260976]).

Distribution in MA \& K: Kazakhstan (N: Akmola prov.; NE: E Kazakhstan prov.; Central: Kzyl-Orda prov.; S: S Kazakhstan prov., Zhambyl prov.; SE: Almaty prov.), Kyrgyzstan (Batken prov., Jalal-Abad prov., Ysyk-köl prov., Osh prov., Naryn prov., Talas prov., Chüh prov.), Tajikistan (Kŭhistani Badakhshan, Sughd prov., Khatlon prov., Dushanbe prov.), Uzbekistan (Kara-kalpak, Buxoro prov., Navoi prov., Dzhizak prov., Samarqand prov., Qashqadarya prov., Surxondarya prov., Sirdarya prov., Toshkent prov., Namangan prov., Fergona prov.), Turkmenistan (Balkan prov., Akhal prov., Lebap prov., Mary prov.).

Distribution in other Asian countries: Russia, China, India, Pakistan, Afghanistan, Iran, Azerbaidzhan, Georgia, Armenia, Turkey, Iraq, Lebanon, Syria, Israel, Jordan, Cyprus

Distribution area: Asia (Norhtrn, Central, Eastern, South-Western and Mediterranean), Europa, Africa, N. America (adv.).

Vicatia atrosanguinea (Kar. et Kir.) P. K. Mukh. et Pimenov, 1991, Feddes Repert. 102(5-6): 377, fig. 1. $\equiv$ Carum atrosanguineum Kar. et Kir. 1842, Bull. Soc. Imp. Naturalistes Moscou 15: 359.

Type: KAZAKHSTAN. "In umbrosis muscosis rupium summarum alpium Alatau ad fl. Lepsa et Sarchan, 1841, Karelin \& Kirilov 1510" (lectotype LE! [LE00051484], designated by Gubanov et al., 1998); isolectotypes LE! [LE00051485LE00051491], MW! [MW0593807], TK).

= Pimpinella saxifraga L. var. dissectifolia C. B. Clarke, 1879, in Fl. Brit. India (J. D. Hooker) 2: 685., nom illeg. (Art. 53.1), Boiss. (1872).

Type: INDIA. 'Kashmir, Tilail, alt. 13000', Clarke" (syntype K?); PAKISTAN. Karakorum, alt. 14000', Clarke" (syntype K?).
= Tongoloa wolffiana Fedde ex H. Wolff, 1929, Repert. Spec. Nov. Regni Veg. 27: 181.

= Vicatia wolffiana (Fedde ex H. Wolff) C. Norman, 1940, J. Bot. 78: 229.

Type: INDIA. "N.W. Himalaya, Tihri-Garhwal, Ourie Gadh, in Nila valley, 14-15000', Duthie 1134" (lectotype K!, designated by Pimenov in Pimenov \& Ostroumova 2012: 149; isolectotypes BM, CAL, DD, G! [G00367407]; "Alampi-la, 14000', Duthie 12176" (syntypes CAL, DD).

Distribution in MA \& K: Kazakhstan (NE: E Kazakhstan prov.; S: S Kazakhstan prov.; SE: Almaty prov.), Kyrgyzstan (Batken prov., JalalAbad prov., Ysyk-köl prov., Naryn prov., Osh prov., Talas prov., Chüh prov.), Tajikistan (Kŭhistani Badakhshan, Sughd prov., Khatlon prov., Dushanbe prov.).

Distribution in other Asian countries: Russia, China, India, Afghanistan.

Distribution area: Northern, Central, Eastern and South-Western Asia.

Xanthoselinum alsaticum (L.) Schur 1866, Enum. Pl. Transsilv.: 264.

$\equiv$ Peucedanum alsaticum L., 1762, Sp. Pl. (ed. 2) $1: 354$.

$\equiv$ Selinum alsaticum (L.) Crantz, 1767, Stirp. Austr. Fasc. 3: 117.

$\equiv$ Cnidium alsaticum (L.) Spreng., 1813, Neue Schr. Naturf. Ges. Halle 2(1) (Pl. Umb. Prodr.): 40.

$\equiv$ Ligusticum alsaticum (L.) Link, 1821, Enum. Hort. Berol. Alt. 1: 275.

$\equiv$ Cervaria alsatica (L.) Gaud., 1828, Fl. Helv. 2: 327 .

Type: ICON. in: C. Bauhin, Prodr. Theatri Bot., ill. 77 (Daucus alsaticus). 1620 (lectotype; designated by Reduron \& al. 1997: 97).

= Taeniopetalum peucedanoides Bunge, 1852, Beitr. Fl. Russl. (Lehm. Rel. Bot.): 127.

Type: EUROPE, RUSSIA. "Auf der Linie zwischen Orenburg und Orskaja, 22 VIII 1840, Lehmann 540" (syntypes P! [P00731602], W!); Zwischen Ufa und Sterlitamak, VII 1840, Lehmann (syntypes P! [P00731600, P00731601]).

= Peucedanum lubimenkoanum Kotov, 1938, Journ. Inst. Bot. Acad. Sci. Ukraine 26-27: 187.

Type: EUROPE, RUSSIA. "Ostrogorsk prope Woronezh [Ostrogorskyi yezd] 1849, Czernjaev" (holotype KW!).

Distribution in MA \& K: Kazakhstan (W: W Kazakhstan prov., Atyrau prov., Aktobe prov.; N: Kostonai prov., Akmola prov., N Kazakhstan prov., Pavlodar prov.; Central: Karagandy prov.). 
Distribution in other Asian countries: Russia, Georgia.

Distribution area: Asia (Northern and Central), Europa.

Note: The specimen of Taeniopetalum peucedanoides kept in $\mathrm{W}$ under the number 540 is not Xanthoselinum alsaticum, but Angelica sylvestris L.

Zeravschania kopetdaghensis Pimenov et Kljuykov, 2013, Phytotaxa 130(1): 28, fig. 3, 4.

Type: TURKMENISTAN. "Turkmen SSR, Kopet Dagh cenrtalis, pars orientalis, angustiae Kelte-Czinar, in rupibus. 27 VI 1948, Blinovski" (holotype LE!; isotypes LE!).

Distribution in Asia: Turkmenistan (Akhal prov.) Endemic.

Distribution area: South-Western Asia.

Zeravschania regeliana Korovin, 1948, Bot. Mater. Gerb. Inst. Bot. Zool. Akad. Nauk Uzbeksk. SSR 12: 28.

Type: TAJIKISTAN. "Vall. fl. Zeravschan, inter pag. Kschtut et [lacum] Kuli-Kalon, 1882, A. Regel" (holotype LE! [LE00052184]; isotypes LE [LE00052178-LE00052183， LE00052185LE00052189]).

Distribution in Asia: Tajikistan (Sughd prov., Dushanbe prov.), Uzbekistan (Qashqadarya prov., Surxondarya prov.). Endemic.

Distribution area: South-Western Asia.

Zeravschania scabrifolia Pimenov, 1983, in Vvedensky (ed.), Opred. Rast. Sred. Azii 7: 375, 274.

Type: TAJIKISTAN. "Tadjikistania, jugum Petri Primi, pars occidentalis, declivum septentrionale prope trajectum Kamchirak, 2700 m, 13 VIII 1975, Pimenov 1127" (holotype MW! [MW0593839]; isotypes LE! [LE00052190], MW! [MW0593828]).

Distribution in Asia: Tajikistan (Dushanbe prov.). Endemic.

Distribution area: South-Western Asia.

Zosima absinthiifolia (Vent.) Link, 1821, Enum. Hort. Berol. Alt. 1: 274, "apsinthifolia".

$\equiv$ Heracleum absinthiifolium Vent., 1803, in Choix P1.: tab. 7, "absintifolium".

$\equiv$ Tordylium absinthiifolium (Vent.) Pers. 1805, Syn. P1. 1: 341, "absintifolium".

$\equiv$ Zosima orientalis Hoffm., 1814, Gen. P1. Umbell.: 148, tab. 1 B, fig. 7, nom. illeg. (Art. 52.1).

$\equiv$ Pastinaca absinthiifolia (Vent.) Calest., 1905, Webbia 1: 247.
Type: "Hort. Cels, Ventenat" (lectotype G [G00341310]; designated by M. W. Callmander in $\mathrm{G}$ and here).

= Heracleum tomentosum Sm. 1806, in Fl. Graec. Prodr. (Sibthorp) 1(1): 192.

Type: EUROPE. "In Graecia at locus non memoratur, Sibthorp" (holotype OXF!).

$=$ Zosima absinthiifolia (Vent.) Link var. albiflora Fisch. et al. (eds.), 1840, Index Sem. Horti Petrop. 7: 58.

= Zosima absinthiifolia (Vent.) Link var. viridiflora Fisch. et al. (eds.), 1840, Index Sem. Horti Petrop. 7: 59.

= Pichleria cruciata Stapf et Wettst. 1886, Denkschr. Akad. Wien 2: 324.

Type: IRAN. "Prope Rustemabad; in solo porpyritico, 23 IV 1882, Polak \& Pichler" (lectotype WU! [WU0025982]; designated here by Pimenov; isolectotype G [G00359613]); "In agris ad Sepujin prope Kaswin circ. 1600 m. 04 V 1882. Polak \& Pichler" (syntypes WU [ WU0025981-WU0025983, WU0073004, W0073005]).

= Pichleria pallidiflora Stapf et Wettst. 1886, Denkschr. Akad. Wien 2: 324.

Type: IRAN. "In rupestribus ad Hissar in itinere ad Hamadan. 10 V 1882. Polak \& Pichler" (holotype WU! [WU0025984]).

= Zosima absinthiifolia (Vent.) Link var. tereticaulis Kuntze, 1887, Trudy Imp. S.-Peterburgsk. Bot. Sada 10(1): 193.

Type: TURKMENISTAN. "V 1886, Kuntze" (holotype NY [NY00406310]).

= Zosima absinthiifolia (Vent.) Link f. stenocarpa Kuntze, 1887, Trudy Imp. S.-Peterburgsk. Bot. Sada 10(1): 193.

Type: TURKMENISTAN. "V 1886, Kuntze" (holotype NY [NY00406309]).

= Zosima transcaspica Gand. 1918, Bull. Soc. Bot. France: 32.

Type: TURKMENISTAN. "Transcaspia, in steppis ad Krasnovodsk, 21 IV 1901, Sintenis 1530" (lectotype P; designated here by Pimenov; isolectotypes L, LD [LD1549323, LD1549383], LE! [LE00052214, LE00052215]).

=Zosima iranica Manden. 1953, Trudy Tbilissk. Bot. Inst. 15: 161.

Type: IRAN. "Chorassan, inter Shahrud et Nishapur, VI 1858, Bunge" (holotype LE!).

Distribution in MA \& K: Kazakhstan (W: Aktobe prov., Mangystau prov.; Central: Karagandy prov., Kzyl-Orda prov.; S: Zhambyl prov.), Uzbekistan (Kara-kalpak, Khorezm prov.), Turkmenistan (Balkan prov., Akhal prov., Mary prov.). 
Distribution in other Asian countries: Russia, India, Pakistan, Afghanistan, Azerbaidzan, Georgia, Armenia, Turkey, Iraq, Saudi Arabia, Syria, Jordan, Egypt (Sinai), Cyprus.

Distribution area: Asia (Northern, Central, South-Western and Mediterranean), Europa, Africa.

Zosima korovinii Pimenov, 1976, Byull. Glavn. Bot. Sada 101: 45.

Type: KYRGYZSTAN. "Asia Media, jugum Kirghisicum, pars orientalis, vallis fluvii Tschu, angustis Boam. 27 V 1970. Pimenov 11" (holotype LE!; isotypes B!, GB!).

- Zosima tordyloides auct. non Korovin: Schischk., 1951, in Schischkin (ed.), Fl. URSS 17: 267.

Distribution in MA \& K: Kazakhstan (S: S Kazakhstan prov., Zhambyl prov.; SE: Almaty prov.), Kyrgyzstan (Jalal-Abad prov., Ysyk-köl prov., Naryn prov., Osh prov., Talas prov., Chüh prov.), Tajikistan (Sughd prov.), Uzbekistan ((Toshkent prov.).

Distribution in other Asian countries: China.

Distribution area: Central \& South-Western Asia.

\section{Conclusion}

Umbelliferae biodiversity within the borders of Middle Asia and Kazakhstan can be characterized by the following numbers. In the overall territory of Kazakhstan, Kyrgyzstan, Tajikistan, Uzbekistan and Turkmenistan, 107 genera/459 species/204 endemic species of the Umbelliferae, have currently been registered. 82 genera/211 species/28 endemic species are distributed in Kazakhstan, 68 genera/200 species/18 endemic species in Uzbekistan, 65 genera/ 192 species/29 endemic species in Kyrgyzstan, 65 genera/176 species/20 endemic species in Tajikistan, and 51 genera/122 species/9 endemic species in Turkmenistan. Despite some difference in numbers, three states of mountainous Middle Asia (Uzbekistan, Kyrgyzstan and Tajikistan) plus Kazhakhstan, the southern part of which geographically and floristically forms one phytochorion with these three republics are similar in their sets of Umbelliferae biodiversity, whereas Turkmenistan is considerably different. Only its smaller part, i.e., the western slope of the Kuhitang Range, could be regarded within mountainous Middle Asia.

Similar relationships are revealed if one compares the lists of five biggest Umbelliferae genera by country: In each of them, the genus Ferula will come first, with 48 species in Kazakhstan, 47 species in Uzbekistan, 41 species in Tadjikistan, 37 species in Kyrgyzstan, and only 19 species in Turkmenistan. The genus Seseli is the second in Kazakhstan (29 sp.), Kyrgyzstan (23 sp.), Uzbekistan (20 sp.), and Tajikistan (13 sp.), but not in Turkmenistan, where Elwendia is the second (11 sp.). Interestingly, the next three genera - turn out to be different: SchrenkiaBupleurum-Aulasospermum in Kazakhstan, Bupleurum - Elaeosticta - Aulacospermum in Kyrgyzstan, Elwendia - Elaeosticta - Prangos in Uzbekistan, Elaeosticta - Semenovia - Elwendia in Tajikistan, and Elaeosticta - Dorema - Pimpinella in Turkmenistan.

The distribution of Umbelliferae specific diversity across the five states' provinces is uneven.

In Kazakhstan, the number of species of the family in southern and eastern, largely mountainous, provinces is much higherthan in its western and central part, that is mainly flat or with low-mountains. Thus, in the region of W Kazakhstan there are 30 species of Umbelliferae in Western Kazakhstan province, 12 species - in Atyrau, 46 species in Aktobe, and 17 species in Mangustau. Similar low numbers are characteristic of $\mathrm{N}$ Kazakhstan: 27 species in North-Kazakhstan province, 37 species in Kostonai, 27 species in Pavlodar, and 42 species in Akmola. Central Kazakhstan occupies an intermediate position, with rather poor Kzyl-Orda province (35 species) and richer, enormous, and predominantly low-mountainous Karagangy province (69 species). Largely mountainous provinces of South-Eastern and Southern Kazalkhstan, floristically forming part of Mountainous Middle Asia are characterized by a predictably high biodiversity in Umbelliferae (South-Kazakhstan province - 123 species, Zhambyl province - 105 species, Almaty province 90 species). There were 89 species registered in NE Kazakhstan, including those not distributed in other Kazakhstan provinces. Among these differential species there are northern (boreal) elements, common with Siberian flora, Central-Asian plants, shared with adjacent the Chinese Xinjiang-Uygur Autonomous Region, and some truly MiddleAsian species, reaching their northern border of distribution on the southern slope of the Tarbagatai Ridge.

Endemic genera (2): Karatavia Pimenov \& Lavrova, Tschulaktavia Bajtenov ex Pimenov et Kljuykov

Endemic species (28): Autumnalia botschantzevii, Dorema karataviense, Eryngium karatavicum, Ferula glaberrima, F. gypsacea, 
F. leucographa, F. malacophylla, F. pachyphylla, $F$. sugatensis, $F$. taukumica, $F$. xeromorpha, Hyalolaena tschuiliensis, Karatavia kultiassivii, Ledebouriella seseloides, Pachypleurum altaicum, Pilopleura goloskokovii, Prangos dzhungarica, P. equisetoides, P. lachnantha, Schrenkia congesta, $S$. involucrata, S. kultiassovii, S. papillaris, Schtschurowskia margaritae, Seseli betpakdalense, S. mironovii, Sphaenolobium korovinii, Tschulaktavia saxatilis.

In 7 provinces of Kyrgyzstan, the difference in the number of Umbelliferae species is not as significant as in Kazakhstan. This is mainly due to the fact that they are all mountainous, with the largest peaks exceeding $4000 \mathrm{~m}$. At the same time, in most provinces there are foothills and even plains. Only Ysyk-köl and Naryn provinces are fully midmountainous and highland regions. Their flora is not so rich as in remaining provinces (Ysyk-köl prov. 49 species of Umbelliferae and Naryn prov - 50 species, against 122 species in Jalal-Abad prov, 97 species in Osh prov., and 94 species in Batken prov. It should be noted also that florists include significant eastern parts of Ysyk-köl and Naryn provinces into the Central Asian phytochorion the poorest one in Umbelliferae diversity. Chüh and Talas provinces, with 70 and 64 Umbelliferae species respectively, occupy an intermediate position.

Endemic species (29): Aulacospermum alaicum, A. gracile, A. plicatum, A. tenuisectum, Bupleurum ferganense, B. isfairamicum, Elwendia sary-cheleki, Ferula alaica, F. czatkalensis, $F$. fedoroviorum, F. ferganensis, F. inciso-serrata, F. pimenovii, F. subtilis, Mogoltavia narynensis, Paulita alaica, Schrenkia alaica, S. hebecarpa, S. pulverulenta, Semenovia alaica, S. vaginata, Seseli alaicum, S. eryngioides, $S$. galioides, S. giganteum, S. kaschgaricum, S. korshinskyi, S. luteolum, S. tenellum.

A similar situation exists in Tajikistan that currently includes four administrative-territorial subdivisions, whose names and borders changed repeatedly in the past. Three of them are Kühistani Badakhshan (an autonomous province), Sughd, and Khatlon province; the rest of the territory is called "regions of republican subordination" and is here designated as "Dushanbe prov." for short. The number of Umbelliferae species per province differs, but not as sharply as in some other countries of the region. Maximal Umbelliferae diversity was registered for Sughd province - 129 species. This can be accounted for by the fact that the area includes very different territories, both in terms of their present natural condition and geological history - from the arid lowlands of Mogoltau Mts. that make up the Western Tian Shan system, to the highest mountains of Kuhistan, the outskirts of Pamiro-Alai. Slightly inferior to this province of Umbelliferae diversity is Dushanbe prov. (102 species) which shares many species with the above-mentioned Sughd province. Lying even further south, Khatlon province also has a fairly diverse set of the family species (83). Finally, Kühistani Badakhshan consists of two naturally different parts, i.e., the high-mountainous plateau of Pamirs and Tajik Badakhshan proper; between them lies the floristic border separating Middle and Central Asia. In total, there are 74 species of Umbelliferae in Kühistani Badakhshan, most of which are distributed in the latter's western part.

Endemic genus: Kafirnigania.

Endemic species (20): Aulacospermum ikonnikovii, Bupleurum linczevskii, Ferula conocaula, F. decurrens, F. equisetacea, F. eugenii, $F$. hissarica, F. linczevskii, $F$. mogoltavica, F. seravschanica, F. violacea, Hyalolaena melanorhiza, Kafirnigania hissarica, Paulita alpina, Semenovia dissectifolia, S. furcata, S. pulvinata, Seseli rimosum, S. sclerophyllum, Zeravschania scabrifolia.

Unlike Kyrgyzstan and Tajikistan, 12 provinces of Uzbekistan, as well as the Karakalpak Autonomous Republic, are very different in natural conditions, with most of the country being a flat desert (Kyzyl-kum, the eastern part of the Usturt Plateau, etc.) or occupied by irrigated cotton fields and orchards. Umbelliferae biodiversity is concentrated in mountainous areas. There is a well-defined correlation between the province's relief and the number of Umbelliferae species. The greatest number of species is observed in provinces whose territory boasts the entire altitude profile, from the foothills to the alpine belt. These are primarily Toshkent, Qashqadarya, and Surxondarya provinces with 98, 89, and 87 Umbelliferae species, respectively. Similar data were obtained for Samarqand and Dzhizak provinces (76 and 75 species of the family). At the opposite end of the range are Sirdarya, Andijon, and Khorezm provinces, with 21, 14 and 7 species, respectively.

Endemic genera: Kuramosciadium, Sphaerosciadium.

Endemic species (19): Autumnalia innopinata, Cephanopodum hissaricum, Elwendia angreni, Elwendia bucharica, Elwendia ugamica, Ferula helenae, F. kyzylkumica, F. nuratavica, F. pratovii, Galagania neglecta, Korshinskia tianschanica, 
Kuramosciadium corydalifolium, Lomatocarpa multivittata, Oenanthe fedtschenkoana, Seseli desertorum, S. merkolowiczii, Spaenolobium tenuisectum, Sphaerosciadium denaense.

Like most of the areas under consideration Turkmenistan undoubtedly belongs to the vast phytochorion of South-West Asia, but is closer to Iran in in terms of flora. Only a small area bordering Uzbekistan on the western slope of the Kuhitang Range belongs to Mountainous Middle Asia. The diversity of Umbelliferae species is confined primarily to the Kopetdagh and Balchan Ridges (prov. Akhal- 78 species, prov. Balkan - 68 species). Somewhat fewer Umbelliferae species are registered in prov. Lepab (52 species), which includes parts of Kuhitang Ridge, and in prov. Mary (41 species), with the Badhyz lowlands and the foothills of the Parapamiz Ridge in Afghanistan. Desert prov. Daşoguz differs from the above mentioned by the lowest number of known Umbelliferae species (8).

Endemic species (9): Dorema balchanorum, Elaeosticta korovinii, E. platyphylla, Elwendia kopetdaghensis, Ferula plurivittata, Galagania margiana, Hyalolaena transcaspica, Pimpinella bobrovii, Zeravschania kopetdaghensis.

The biggest genera in the Umbelliferae of Middle Asian \& Kazakhstanian flora are Ferula (100 species), Seseli (49 species), Bupleurum, Elaeosticta \& Elwendia (all with 18 species each), Prangos (17 species), Semenovia (16 species), Aulacospermum
\& Schrenkia (each with 13 species), and Hyalolaena (10 species).

16 genera are endemic to Middle Asia and Kazakhstan - Astomatopsis, Autumnalia, Fergania, Kafirnigania, Karatavia, Komarovia, Kuramosciadium, Lipskya, Mogoltavia, Paulita, Pilopleura, Schtschurowskia, Sclerotiaria, Sphaenolobium, Sphaerosciadium, and Tschulaktavia. The following 6 genera can be classified as subendemics - Ledebouriella, Mediasia, Oedibasis, Ormopterum, Pastinacopsis, and Schrenkia.

\section{Acknowledgements}

We are grateful to the curators of LE (prof. D. V. Geltman) and TASH (prof. K. Sh. Tojibaev), as well as to staff of LE, MW, P, TASH, W and other mentioned herbaria for use of their facilities. Author is thankful to Dr. Alexander Sennikov (Helsinki) and the redactor of the article Dr. Dmitry German (Barnaul) for their valuable comments in complicated cases of botanical nomenclature.

\section{Funding}

The work is supported by the Russian Foundation of Basic Research (grant 19-04-00370).

\section{ORCID}

Michael Pimenov http://orcid.org/0000-00022192-9965

\section{REFERENCES/ЛИTEPATУPA}

Al-Eisawi D., Jury S. L. 1988. A taxonomic revision of the genus Tordylium L. (Apiaceae). Botanical Journal of the Linnean Society 97(4): 357-404.

Baitulin I. O., Kotykhov Yu. A. 2011. Flora sosudistykh rasteniy Kazakhstanskoro Altaya [Flora of vascular plants of Kazakhstan Altai]. Almaty: Institut botaniki i fitointroduktsii AN Respubliki Kazakhstan. 158 pp. [In Russian] (Байтулин И. О., Котухов Ю. А. Флора сосудистых растений Казахстанского Алтая. Алматы: Институт ботаники и фитоинтродукции АН Республики Казахстан, 2011. 158 с.).

Batarda Fernandes R. 1967. Sur l'identification et la typification de Caucalis daucoides L. et de Caucalis grandiflora L. Boletim da Sociedade Broteriana, sér. 2. 41: 395-407.

Boissier E. 1872. Umbelliferae. In: Flora orientalis sive, enumeratio plantarum in Oriente a Graecia et Aegypto ad Indiae fines nucusque observatae. 2. Genève, Basele, Lyon: Publisher H. Georg. Pp. 819-1090.

Boissier E. 1888. Umbelliferae. In: Flora Orientalis Supplementum. Eds. R. Buser. Genève, Basele: H. Georg. Pp. 251-272.

Borszczow E. 1860. Die Pharmazeutisch-wichtigen Ferulaceen der Aralo-Caspishen Wüste, nebst allgemeinen Untersuchungen über die Abstammung der im Handel vorkommenden Gummiharze: Asa foetida, Ammoniacum und Galbanum. Mémoires de l'Académie Impériale des Sciences de Saint-Petersbourg Divers Savans, 7 ser. 3(8): 40.

Breckle S.-W., Hedge I. C., Rafiqpoor M. D. 2013. Vascular plants of Afghanistan: an augmented checklist. Bonn: Sciencia Bonnensis. 597 pp.

Bunge A. A. 1843. Eremodaucus. In: Delectus Seminum quae in horto botanico Dorpatensi pro mutia commentatione offeruntur e collectionibus annorum 1837-1843. Dorpat. P. [6].

Bunge A. A. 1849. Heracleum Lehmannianum Bge. In: Delectus seminum e collectione anni 1849, quae hortus botanicus Dorpatensis pro mutia commutatione offeruntur: unpaged [2]. Dorpat: Yur'ev. 
Bunge A. A. 1852. Alexandri Lehmann reliquiae botanicae, sive Enumeratio plantarum ab Alexandro Lenmann in itinere per regiones uralensi-caspicas, deserta Kirghisorum, Transoxanam et Sogdiamam annis 1839-1842 peracto collectarum Separat. St. Peterbourg. Pp. 1-369.

Bunge A. A. 1854. Beitrag zur Kenntniss der Flor Russlands und der Steppen Central-Asiens (Alexandri Lehmann reliquiae botanicae, sive Enumeratio plantarum ab Alexandro Lenmann in itinere per regiones uralensi-caspicas, deserta Kirghisorum, Transoxanam et Sogdiamam annis 1839-1842 peracto collectarum. St. Petersbourg. Pp. $177-536$.

Chamberlain D. F. 1987. Ferulago. In: Flora Iranica. Ed. K. H. Rechinger. Vol. 162. Graz: Akademische Drucku.-Verlagsanstalt. Pp. 427-434.

Chamberlain D. F., Rechinger K. H. 1987. Ferula. In: Flora Iranica. Ed. K. H. Rechinger. Vol. 162. Graz: Akademische Druck-u.-Verlagsanstalt. Pp. 387-426.

Czubarov I. N. 2003. Apiaceae (Umbelliferae). In: Opredelitel rasteniy Altayskogo kraya [Manual of plants of Altai territory]. Novosibirsk: Izdatelstvo SO RAN. Pp. 291-311. [In Russian] (Чубаров И. Н. Apiaceae (Umbelliferae) // Определитель растений Алтайского края. Под ред. И. М. Красноборова. Новосибирск: Изд-во СО РАН, филиал Гео, 2003. С. 291-311).

Dawit A. 1992. Systematic studies in Pimpinella L. (Umbelliferae) from tropical Africa. Botanical Journal of the Linnean Society 110: 327-372.

Engstrand L. 1987. Pimpinella. In: Flora Iranica. Ed. K. H. Rechinger. Vol. 162. Graz: Akademische Druck-u.-Verlagsanstalt. Pp. 311-333.

Fedoronchuk N. M. 1979. In: Vinogradova V. M., Fedoronchuk N. M. Stenocoelium popovii V. Vinogr. et Fedoronchuk (Apiaceae) - species nova ex Asia Centralis. Novosti sistematiki vysshikh rasteniy [Novit. Syst. Pl. Vasc.] 23: 95-101. [In Russian] (Федорончук Н. М. // Виноградова В. М., Федорончук Н. М. Stenocoelium popovii V. Vinogr. et Fedoronchuk (Apiaceae) - новый вид из Центральной Азии // Новости сист. высш. раст., 1979. Т. 23. C. 95-101).

Fedoronczuk N. M. 1983. Sistematika, geografiya i filogeniya rodov Trinia, Rumia, Ledebouriella [Systematics, geography and phylogeny of the genera Trinia, Rumia, and Ledebouriella]. Kiev: Naukova Dumka. 160 pp. [In Russian] (Федорончук Н. M. Систематика, география и филогения родов триния, румия, ледебуриелла. Киев: Наукова Думка, 1983. 160 с.).

Fedoronchuk N. M. 2005a. Typification and critical analysis of the species of family Apiaceae Lindl., described from territory of Ucraina: genera Anthriscus Pers. - Ferulago C. Koch. Ucr. Botan. Zhurn. 62(1): 19. [In Russian] (Федорончук Н. М. Типификация и критический анализ описанных с территории Украины видов семейства Apiaceae Lindl.: genera Anthriscus Pers. - Ferulago C. Koch // Укр. ботан. журн., 2005а. Т. 62, № 1. С. 19.

Fedoronchuk M. M. 2005b. Typification and critical analysis of the species of family Apiaceae Lindl., described from territory of Ucraina. Genera Gasparinia Bertol. - Rumia Hoffm. Ucr. Bot. Zhurn. 62(5): 640-648. [In Ukrainian] (Федорончук M. М. Типифікація та критичний аналіз описаних з території України видів родини Аріасеае Lindl.: роди Gasparinia Bertol. - Rumia Hoffm. // Укр. ботан. журн., 2005б. Т. 62, № 5. С. 640-648).

Fedtschenko B. A. 1899. Notes sur quelques espèces du genre Prangos Lindl. Bulletin de l'Herbier Boissier 7 : 178-181.

Fedtschenko B. A. 1902. Material for the flora of Schugnan. Trudy Botanicheskoro Muzeya Imperatorskoy Akademii Nauk 1: 110-170. [In Russian] (Федченко Б. A. Материал для флоры Шугнана // Тр. Бот. музея Имп. Акад. наук, 1902. T. 1. C. 110-170).

Fedtschenko B. A. 1915. Rastitelnost Turkestana. Illustrirovannoye posobiye dlya opredeleniya rasteniy, dikorastushikh $v$ Turkestanskom kraye i Kirgizskikh stepyakh [Vegetation of Turkestan. An illustrated guide for determination of plants, wild growing in Turkestan and Kirghiz steppes]. Petrograd. 824 pp. [In Russian] (Федченко Б. А. Растительность Туркестана. Иллюстрированное пособие для определения растений, дикорастущих в Туркестанском крае и Киргизских степях. Петроград, 1915. 824 с.).

Fedtschenko O. A., Fedtschenko B. A. 1909. Conspectus florae Turkestanicae [Conspect of Turkestanian flora]. Pt. 3. Yur'ev: K. Matisen. 156 pp. [In Russian] (Федченко О. А., Федченко Б. А. Конспект флоры Туркестана. Ч. 3. Юрьев: Матисен, 1909. 156 с.).

Fischer F. E. L., Meyer C. A. 1841. Enumeratio plantarum novarum a cl. Schrenk lectarum Vol. 1. Petropoli: 113.

Fischer F. E. L., Meyer C. A. 1842. Enumeratio plantarum novarum a cl. Schrenk lectarum. Vol. 2. Petropoli: 77 p.

Fischer F. E. L. [\& Meyer C. A.]. 1845. Diagnoses plantarum novarum, a cl. Al. Schrenk anno 1844 in Songaria lectarum. Manipulus ultimis. Bulletin de la Classe Physico-Mathématique de l'Académie Impériale des Sciences de Saint-Petersbourg 320(68): 305-309.

Flora of Uzbekistan. 2017. Vol. 2. (Primulaceae, Plumbaginaceae, Scrophulariaceae, Linderniaceae, Mazaceae, Sphenocleaceae, Campanulaceae). Ed. A. N. Sennikov. Tashkent: Navro'z Publishers. xii +200 pp. [In Russian] (Флора Узбекистана. Т. 2. Ред. А. Н. Сенников. Ташкент: Изд-во «Навруз», 2017. хіi + 200 с.). 
Flora of Uzbekistan. 2019. Vol. 3. (Plumbaginaceae, Caprifoliaceae, Gentianaceae, Solanaceae, Bignoniaceae, Menyanthaceae, Oleaceae). Ed. A. N. Sennikov. Tashkent: Ma’naviyat Publishers. xii + 201 pp. [In Russian] (Флора Узбекистана. Т. 3. Ред. А. Н. Сенников. Ташкент: Изд-во «Маънавият», 2019. хіi + 201 с.).

Franchet A. 1883. Plantes du Turkestan. Annales des Sciences Naturelles (Paris), sér. 7, Bot. 16: $280-336$.

Geldykhanov A. M. 1985. The new taxa of the genus Bunium (Apiaceae) from Turkmenia. Bot. Zhurn. (Moscow \& Leningrad) 70(11): 1558-1565. [In Russian] (Гельдиханов А. М. Новые таксоны рода Випіuт (Аріасеае) из Туркмении // Бот. журн., 1985. Т. 70, № 11. С. 1558-1565).

Geldykhanov A. M. 1992. Zontichnyye flory Turkmenistana [Umbelliferae of Turkmenistan flora] Ashkhabad: Ylym. 188 pp. [In Russian] (Гельдиханов A. М. Зонтичные флоры Туркменистана. Ашхабад: Ылым, 1992. 188 c.).

Goloskokov V. P. 1963. Herbarium typorum plantarum Kasachstanicae. Trudy Instituta botaniki Akademii Nauk Kazakhskoy SSR [Proceedings of the institute of botany of the academy of sciences of the Kazakh SSR 1: 1-68. [In Russian] (Голоскоков В. П. Гербарий типов растений Казахстана // Труды Института ботаники Академии Наук Казахской ССР, 1963. Т. 1. С. 1-68).

Grubov V. I. 1959. Opyt botaniko-geograficheskogo rayonirovaniya Centralnoy Azii [The experience of botanicalgeographical regionalization of Central Asia]. Leningrad. 77 pp. [In Russian] (Грубов В. И. Опыт ботанико-географического районирования Центральной Азии. Л., 1959. 77 с.).

Gubanov I. A. 1993. Katalog tipovykh obrazstov sosudistykh rasteniy gerbariya Moskovskogo universiteta (MW) [Catalogue of authentic specimens of vascular plants of the Moscow university Herbarium (MW)]. Moscow. MGU Publishers. 160 pp. [In Russian] (Губанов И. А. Каталог типовых образцов сосудистых растений гербария Московского университета (MW). М.: Изд-во МГУ, 1993. 160 с.).

Gubanov I. A., Bagdasarova T. V., Balandina T. P. 1998. Scientific heritage of the prominent Russian florists G. S. Karelin and I. P. Kirillov. Moscow: MGU Publishers. 95 pp. [In Russian] (Губанов И. А., Багдасарова Т. В., Баландина Т. П. Научное наследие выдающихся русских флористов Г. С. Карелина и И. П. Кириллова. М.: Изд-во МГУ, 1998. 95 с.).

Güner A., Aslan S., Ekim T., Vural M., Babaç M. T. (eds.). 2012. Türkiye Bitkileri Listesi (Damarlı Bitkiler). Istanbul: Nezahat Gökyiğit Botanik Bahçesi ve Flora Araşturmaları Derneği Yauını. 1290 pp. [In Turkish]

Hedge I. C., Lamond J. M. 1972. Cicuta. In: Flora of Turkey and the East Aegean Islands Ed. P. H. Davis. Vol. 4. Edinburgh: University Press. P. 425.

Hedge I. C., Lamond J. M. 1987. Chaerophyllum, Physocaulis. In: Flora Iranica. Ed. K. H. Rechinger Vol. 162. Graz: Akademische Druck-u.-Verlagsanstalt. Pp. 86-95, 100-101.

Heywood V. H. 1960. On the identity of Caucalis platycarpos L. and C. daucoides L. Agronomia Lusitana 2(2): $11-13$.

Jafri S. M. H. 1985. Apiaceae. In: Flora of Lybia. Vol. 117. Eds. S. M. H. Jafri, A. El-Gadi. Tripoli: Al Faateh University. Pp. 1-159.

Jansen P. C. M. 1981. Spices, condiments and medicinal plants of Ethiopia: Their taxonomy and agricultural significance. Wageningen: Centre for Agricultural Publishing and Documentation. Pp. 1-32.

Jarvis C. E., Barrie F. R., Allan D. M., Reveal J. L. 1993. A list of Linnean generic names and their types. Regnum Vegetabilis 127: 1-100.

Jarvis $\boldsymbol{C}$. E., Knees S. G. 1988. Linnean names in the genus Athamanta L. (Umbelliferae: Apioideae) and their typification. Taxon 37(2): 448-453.

Jarvis C. E., Reduron J.-P., Spencer M. A., Cafferty S. 2006. Typification of Linnean plant names in Apiaceae. Taxon 55(1): 207-216.

Jonsell B., Jarvis $\boldsymbol{C}$. E. 2002. Lectotypifications of Linnean names for Flora Nordica (Brassicaceae-Apiaceae). Nordic Journal of Botany 22(1): 67-86. DOI: 10.1111/j.1756-1051.2002.tb01625.x

Kamelin R. V. 1973. Florogeneticheskiy analiz yestestvennoy flory Gornoy Spedney Azii [Floregenetic analysis of natural flora of Mountainous Middle Asia]. Leningrad: Nauka. 356 pp. [In Russian] (Камелин Р. В. Флорогенетический анализ естественной флоры горной Средней Азии. Л.: Наука, 1973. 356 с.).

Kamelin R. V. 1977. Taxa nova Umbelliferarum Asiae Mediae, 1. Novosti sistematiki vysshikh rasteniy [Novit. Syst. Pl. Vasc.] 14: 187-192. [In Russian] (Камелин P. В. Новые таксоны среднеазиатских зонтичных. 1 // Новости сист. высш. раст., 1977. Т. 14. С. 187-192).

Kamelin R. V. 1979. Kuhistanskiy okrug gornoy Sredney Asii. Botanico-georgaficheskyy analiz [Districtus Kuhistanicus: Analysis botanico-geographica]. Leningrad: Nauka. 117 pp. [In Russian] (Камелин Р. В. Кухистанский округ горной Средней Азии. Ботанико-географический анализ. Л.: Наука, 1979. 117 с.).

Kamelin R. V. 1990. Flora Syrdarinskogo Karatau. Materialy k floristicheskomu rayonirovaniyu Sredney Azii [Flora of Syrdarya Karatau. Materials for floristic division of Middle Asia]. Leningrad: Nauka. 146 pp. [In Russian] (Камелин P. В. Флора Сырдарьинского Каратау. Материалы к флористическому районированию Средней Азии. Л.: Наука, 1990. 146 с.). 
Kamelin R. V. 1998. Materialy po istorii flory Asii (Altaiskaya gornaya strana) [Materials on the history of flora of Asia (Altai mountain country)]. Barnaul: Altaiskiy gosudarstvennyy Universitet. 239 pp. [In Russian] (Камелин $\boldsymbol{P}$. B. Материалы по истории флоры Азии (Алтайская горная страна). Барнаул: Изд-во АГУ, 1998. 239 с.).

Kamelin R. V. 2017. Flora of the Earth: floristic division of the land. St. Petersburg \& Barnaul: "Pyat plyuus". 128 pp. [In Russian] (Камелин P. В. Флора Земли: флористическое районирование суши. СПб.-Барнаул: «Пять Плюс», 2017. 128 с.).

Kamelin R. V. 2018. Geografiya rasteniy [Plant geography]. St. Petersburg: VVM. 306 pp. [In Russian] (Kameлин P. В. География растений. Учеб. Пособие. СПб.: Изд-во ВВМ, 2018. 306 с.).

Karelin G. S., Kirilov I. I. 1841. Enumeratio plantarum anno 1840 in regionibus altaicis et confinibus collectarum. Bulletin de la Société Imperiale des Naturalistes de Moscou 14(3): 369-459.

Karelin G. S., Kirilov I. I. 1842. Enumeratio plantarum in desertis Songoriae orientalis et in jugo summarum alpium Alatau anno 1841 collectarum. Bulletin de la Société Imperiale des Naturalistes de Moscou 15(2): $321-453$.

Kauffmann N. N. 1870. Euryangium sumbul. A plant that delivers the roots of the Bukhara sumbul. Izv. Imp. Obshch. Lyubit. Estestv. Moskovsk. Univ. 8(1): 387-394. [In Russian] (Кауфманн Н. Н. Euryangium sumbul. Растение, доставляющее корни бухарского сумбула // Изв. Имп. Об-ва Любит. Естеств. Антроп. Этногр., 1870. Т. 8, вып. 1. C. 387-394).

Kauffmann N. N. 1871. Euryangium Sumbul die Mutter-pflanze der bucharischen Sumbulwurtzel. Mémoires de la Société Imperiale des Naturalistes de Moscou 13(3): 253-260.

Khassanov F. O. 2016. Amaryllidaceae. In: Flora Uzbekistana [Flora of Uzbekistan]. Vol. 1. Ed. A. N. Sennikov. Tashkent: Navro’z. Pp. 1-119. [In Russian] (Хасанов Ф. О. Amaryllidaceae // Флора Узбекистана. Т. 1. Ред. А. Н. Сенников. Ташкент: «Навруз», 2016. С. 1-119).

Khasanov F. O., Maltzev I. I. 1990. A new species of the genus Ferula L. from Hissar Mts. Izvěstija Akademii Nauk Uzbekskoj SSR 8: 48-49. [In Russian] (Хасанов Ф. О., Мальцев И. И. Новый вид рода Ferula L. из Гиссарского хребта // Известия Академии наук Узбекской ССР, 1990. Т. 8. С. 48-49).

Khasanov F. O., Maltzev I. I. 1992. A new genus Kamelinia (Apiaceae) from Middle Asia. Uzbekskij Biologičeskij Zhurnal 2: 49-53. [In Russian] (Хасанов Ф. О., Мальцев И. И. Новый род Kamelinia из Средней Азии // Узбекский биологический журнал, 1992. Т. 2. С. 49-53).

Kljuykov E. V. 1983. Conspectus specierum generis Elaeosticta Fenzl (Apiaceae). Novosti sistematiki vysshikh rasteniy [Novit. Syst. Pl. Vasc.] 20: 140-154. [In Russian] (Клюйков Е. В. Конспект видов рода Elaeosticta Fenzl (Apiaceae) // Новости сист. высш. раст., 1983. Т. 20. С. 140-154).

Kljuykov E. V. 2020. Anethum involucrarum. In: Tojibaev K. Sh., Beschko N. Ju., Turginov O. T. et al. An annotated checklist of the endemic Apiaceae of Uzbekistan. Phytotaxa 455(2): 70-94. DOI: 10.11616/phytotaxa.455.2.2

Kljuykov E. V., Pimenov M. G., Tichomirov V. N. 1976. Revision of the genus Aulacospermum (Umbelliferae Apioideae) 2. Review of species. Byull. Moskovsk. Obshch. Isp. Prir., Otd. Biol. 81, 5: 61-68. [In Russian] (Клюйков Е. В., Пименов М. Г., Тихомиров В. Н. Ревизия рода Aulacospermum (Umbelliferae-Apioideae) 2. Обзор видов // Бюл. МОИП. Отд. биол., 1976. Т. 81, вып. 5. С. 61-68).

Kljuykov E. V., Pimenov M. G., Tikhomirov V. N. 1977. Bunium capillifolium Kar. et Kir.: taxonomy, geography, ecology. Nauchn. Dokl. Vyssh. Shkoly. Biol. nauki 10: 137-143. [In Russian] (Клюйков Е. В., Пименов М. Г., Тихомиров В. Н. Таксономия, география и экология Bunium capillifolium Kar. et Kir. // Научные доклады высшей школы. Биол. науки, 1977. № 10. С. 137-143).

Kljuykov E. V., Tojibaev K. Sh., Lyskov D. F. 2018. The genus Elwendia Boiss. (Apiaceae) in the flora of Uzbekistan. Turczaninowia 21, 2: 210-220. [In Russian] (Клюйков Е. В., Тожибаев К. Ш., Лысков Д. Ф. Род Elwendia Boiss. (Apiaceae) во флоре Узбекистана // Turczaninowia, 2018. T. 21, вып. 2. С. 210-220). DOI: 10.14258/turczaninowia.21.2.18

Korovin E. P. 1926. Archangelica tschimganica. In: Schedae ad Herbarium florae Asiae Mediae, fasc. 10. Byulleten Sredne-Asiatskogo Gosudarstvennogo Universiteta 14 (Suppl.): 8-15.

Korovin E. P. 1927. The genus Bunium and its Middle-Asian representatives. Bjulleten Sredneasiatskogo Gosudarstvennogo Universiteta 15: 117-129. [In Russian] (Коровин Е. П. Род Вunium L. и его среднеазиатские представители // Бюл. Среднеазиатск. Унив., 1927. Т. 15. С. 117-129).

Korovin E. P. 1947a. Illustirovannaya monographiya roda Ferula L. [Generis Ferula L. monographia illustrata]. Tashkent: Akademia Nauk Uzbekskoy SSR. 91 pp. [In Russian, Latin] (Коровин Е. П. Иллюстрированная монография рода Ferula L. Ташкент: Акад. Наук Узбек. CCP, 1947. 91 с.).

Korovin E. P. 1947b. Species novae Umbelliferarum Florae Uzbekistanicae. 1. Bot. Mat. Herb. Inst. Bot. Zool. Akad. Nauk Uzbek. SSR 8: 3-24. [In Russian] (Коровин E. П. Новые виды зонтичных флоры Узбекистана. 1 // Бот. Матер. Герб. Инст. Бот. Зоол. Акад. наук Узбек. ССР, 1947. Т. 8. C: 3-24).

Korovin E. P. 1947c. Species novae Umbelliferarum Florae Uzbekistanicae. II. Bot. Mat. Herb. Inst. Bot. Zool. Akad. Nauk Uzbek. SSR 12: 13-32. [In Russian] (Коровин Е. П. Новые виды зонтичных флоры Узбекистана. II. // Бот. Матер. Герб. Инст. Бот. Зоол. Акад. наук Узбек. ССР, 1947. Т. 12. С. 13-32). 
Korovin E. P. 1950a. Umbelliferae. In: Flora Turkmenii [Flora of Turkmenistan]. Vol. 5. Ed. V. V. Nikitin. Ashkhabad: Izdatelstvo Turkmenskogo filiala Akademii nauk SSSR. Pp. 171-250. [In Russian] (Коровин Е. П. Umbellifertae // Флора Туркмении. Под ред. В. В. Никитина. Т. 5. Ашхабад: изд-во Туркм. фил. АН СССР, 1950. С. 171-250).

Korovin E. P. 1950b. Scaligeria DC., Muretia Boiss., Korshinskia Lipsky. In: Flora SSSR [Flora of the USSR]. Ed. B. K. Schischkin. Vol. 16. Moscow \& Leningrad: Publishers of Academy of Sciences of USSR. Pp. 204-218, 415-421. [In Russian] (Коровин Е. П. Scaligeria DC., Muretia Boiss., Korshinskia Lipsky // Флора СССР. Т. 16. Под ред. Б. К. Шишкина. М.-Л.: Изд-во АН СССР, 1950. С. 204-218, 415-421).

Korovin E. P. 1951. Ferula L., Oedibasis K.-Pol., Korovinia Nevski et Vved., Scaphespermum Korov. In: Flora SSSR [Flora of the USSR]. Ed. Schischkin B. K., Vol. 17. Moscow \& Leningrad: Publishers of Academy of Sciences of USSR. Pp. 62-141, 203-208, 211-214. [In Russian] (Коровин E. П. Ferula L., Oedibasis K.-Pol., Korovinia Nevski et Vved., Scaphespermum Korov // Флора СССР. Т. 17. Под ред. Б. К. Шишкина. М.-Л.: Изд-во АН СССР, 1951. C. 62-141, 203-208, 211-214).

Korovin E. P. 1959. Umbelliferae. In: Flora Uzbekistana [Flora of the Uzbekistan]. Vol. 4. Ed. A. I. Vvedensky. Tashkent: Akademiya Nauk Uzbekskoy SSP. Pp. 257-470. [In Russian] (Коровин Е. П. Umbelliferae // Флора Узбекистана. Т. 4. Под ред. А. И. Введенского. Ташкент: Акад. наук Узбек. ССР, 1959. С. 257-470).

Korovin E. P. 1963. Umbelliferae In: Flora Kazakhstana [Flora of the Kazakhstan]. Ed. N. V. Pavlov. Vol. 6. Alma -Ata: Izdatelstvo Akademii nauk Kazakhskoy SSR. Pp. 258-428. [In Russian] (Коровин Е. П. Umbelliferae // Флора Казахстана. Т. 6. Под ред. Н. В. Павлова. Алма-Ата: Изд-во Акад. наук Казахской ССР, 1963. С. 258-428).

Korovin E. P. 1973a. The new taxa of the family Umbelliferae from Pamiro-Alaj (1). Izv. Tadzhiksk. Fil. Akad. Nauk S.S.S.R. 1(50): 14-19. [In Russian] (Коровин Е. П. Новые таксоны семейства зонтичных из Памиро-Алая (сообщ. 1) // Изв. Акад. Наук Таджик. ССР, отд. Естеств. Наук, 1973а. Вып. 1(50). Рр. 14-19).

Korovin E. P. 1973b. The new taxa of the family Umbelliferae from Pamiro-Alaj (2). Izv. Tadzhiksk. Fil. Akad. Nauk S.S.S.R. 2(51): 9-13. [In Russian] (Коровин Е. П. Новые таксоны семейства зонтичных из Памиро-Алая (сообщ. 2) // Изв. Акад. Наук Таджик. ССР, отд. Естеств. Наук, 1973b. Вып. 2(51). С. 9-13).

Korovin E. P. 1975. The new taxa of the family Umbelliferae from Pamiro-Alaj (3). Izv. Tadzhiksk. Fil. Akad. Nauk S.S.S.R. 3(60): 3-8. [In Russian] (Коровин Е. П. Новые таксоны семейства зонтичных из Памиро-Алая (сообщ. 3) // Изв. Акад. Наук Таджик. ССР, отд. Естеств. Наук, 1975. Вып. 3(60). С. 3-8).

Korovin E. P. 1986. Ferula tuberifera. In: Schedae herbarium florae URSS. Vol. 25. Leningrad: Nauka. N 6418. [In Russian] (Коровин E. P. Ferula tuberifera // Список растений гербария флоры СССР, издаваемого Ботаническим институтом им. В. Л. Комарова Академии наук СССР. Т. 25. Л.: Наука, 1986. N 6418).

Korovin E. P., Pimenov M. G., Kinzikaeva G. K. 1984. Umbelliferae. In: Flora Tadzhikskoy SSR [Флора Таджикской СCP]. Vol. 7. Ed. P. N. Ovczinnikov. Leningrad: Nauka. Pp. 10-214. [In Russian] (Коровин Е. П., Пименов М. Г., Кинзикаева Г. К. Umbelliferae // Флора Таджикской ССР. Т. 7. Ред. П. Н. Овчинников. Л.: Наука, 1984. C. 10-214).

Koso-Poljansky B. M. 1913a. Species Umbelliferarum minus cognitae. Russk. Bot. Zhurn. 1-2: 1-10 [In Russian] (Козо-Полянский Б. М. Маллоизвестные виды Umbelliferae // Русск. бот. журн., 1913. Т. 1-2. С. 1-10).

Koso-Poljansky B. M. 1913b. Notes on Umbelliferae. III. Nomenclatural note on Sium lancifolium M. B. Trudy botanicheskogo sada Imperatorskogo Yugyevskogo Universitata 14, 2-3: 105-121. [In Russian] (Козо-Полянский Б. М. Заметки по зонтичным. III. Номенклатурная справка о Sium lancifolium М. В. // Труды Бот. Сада имп. Юрьевск. Унив., 1913. Т. 14. Вып. 2-3. С. 105-121).

Koso-Poljansky B. M. 1914. Observations sur le genre Sium L. sensu DC. Bulletin de la Societe Imperiale des Naturalistes de Moscou 28: 170-186. [In Russian] (Козо-Полянский Б. М. Наблюдения по роду Sium sensu DC.) // Бюл. МОИП, 1914. Т. 28. С. 170-186).

Koso-Poljansky B. M. 1915b. Umbelliferae. In: Flora Aziatskoy Rossii [Flora of Asiatic Russia]. Ed. B. A. Fedtschenko. Vol. 10. Petrograd: Publisher A. Kollins. 39 pp. [In Russian] (Козо-Полянский Б. М. Umbelliferae // Флора Азиатской России. Под ред. Б. А. Федченко. Т. 10. Петроград: Типография А. Э. Коллинс, 1915b. 39 с.).

Koso-Poljansky B. M. 1916a. Sciadophytorum systematis lineamenta. Bull. Soc. Imp. Naturalistes Moscou, s. $n$. 29: 93-221.

Koso-Poljansky B. M. 1916b. Species Umbelliferarum minus cognitae. 2. Věstn. Tiffissk. Bot. Sada. 11(3-4): 136-170. [In Russian] (Козо-Полянский Б. М. О видах из семейства зонтичных // Вестн. Тифлис. бот. сада., 1915. Т. 11, вып. 3-4. С. 136-170).

Koso-Poljansky B. M. 1917a. The new species. Izv imp. Bot. Sada Petra Velikogo 17(1): 109-115. [In Russian] (Козо-Полянский Б. М. Новые виды // Изв. имп. Бот. Сада Петра Великого, 1917. Т. 17, № 1. С. 109-115).

Koso-Poljansky B. M. 1917b. Sciadophytorum systematis lineamenta. Mantissa prior. Bull. Soc. Imp. Naturalistes Moscou, s. n. 30(“1916"): 277-290.

Koso-Poljansky B. M. 1920. Umbelliferae - Pachystereomae of Asiatic Russia. In: Flora Aziatskoy Rossii [Flora of Asiatic Russia]. Ed. B. A. Fedtschenko. Vol. 15, № 1. Pp. 1-120. [In Russian] (Козо-Полянский Б. М. Umbel- 
liferae - Рachystereomae Азиатской России // Флора Азиатской России. Под ред. Б. А. Федченко. Т. 15 , № 1. Петроград: Девятая Государственная типография, 1920. С. 1-120).

Koso-Poljansky B. M. 1921. Species nova. 3. Botanicheskie materialy Gerbarija Glavnogo Botanicheskogo Sada R.S.F.S.R. 2(16-17): 61-68. [In Russian] (Козо-Полянский Б. М. Новые виды. 3 // Бот. матер. Герб. Главн. Бот. Сада Р.С.Ф.С.Р., 1921. Т. 2, № 16-17. С. 61-68).

Koso-Poljansky B. M. 1922. Ferula Litwinowiana sp. n., Peucedanum condensatum K.-Pol. (comb. n.), Phlojodicarpus sibiricus K.-Pol. (comb. n.). In: Fasciculus LIII. Spisok Rasteniy Gerbariya Russkoy Flory Botanicheskogo Muzeya Rossiyskoy Akademii Nauk [Schaedae Herb. Fl. Ross.] 8: 111, 115, 117. [In Russian] (Козо-Полянский Б. M. Ferula Litwinowiana sp. n., Peucedanum condensatum K.-Pol. (comb. n.), Phlojodicarpus sibiricus K.-Pol. (comb. n.). // Список растений Гурбария русской флоры Ботанического музея Российской Академии наук, 1922. Вып. 8. С. 111, 115, 117).

Koso-Poljansky B. M. 1925. A preliminary census of giant-fennels (Ferulae) of Russian Central Asia. Bjul. Obsc. Estestvoisp. Voron. Gosud. Univ. 16(1): 89-96. [In Russian] (Козо-Полянский Б. М. Предварительный обзор видов Ferula Русской Центральной Азии // Бюл. Общ. Естествоиспыт. Воронежск. Гос. Унив., 1925. Т. 16 , № 1. C. 89-96).

Kuzmina L. V. 1962. Use of anatomical characters for classification of the species of genus Prangos Lindl. Bot. Zhurn. (Moscow \& Leningrad) 47(2): 250-264. [In Russian] (Кузмина Л. В. Использование анатомических признаков для классификации видов рода Prangos Lindl. // Бот. журн., 1962. Т. 47, № 2. С. 250-264).

Leute G.-H. 1970. Untersuchungen über den Verwandtschaftkreis der Gattung Ligusticum L. (Umbelliferae). Teil 2. Annalen des Naturhistorischen Museums Wien 74: 457-519.

Linczevski I. A. 1950. Bupleurum L. In: Flora SSSR [Flora of the USSR]. Ed. B. K. Schischikin. Vol. 16. MoscowLeningrad: Publishers of Academy of Sciences of USSR. Pp. 275-349. [In Russian] (Линчевский И. А. Bupleurит L. // Флора СССР. Т. 16. Под ред. Б. К. Шишкина. М.-Л.: Изд-во АН СССР, 1950. С. 275-349).

Linnaeus C. 1753. Species plantarum. Ed. 1. Holmiae [Stockholm]: Impensis Laureritii Salvii. 560 pp.

Lipsky V. I. 1896. Revisio generis Aphanopleura (Umbelliferae). Bull. Acad. Imp. Sci. Sankt-Petersb., ser. 5 4(4): 373-381. [In Russian] (Липский В. И. Ревизия рода Aphanopleura (Umbelliferae) // Изв. имп. Акад. Наук, сер. 5, 1896. T. 4, № 4. C. 373-381).

Lipsky V. I. 1900. Materials for the flora of Middle Asia. I. Acta Horti Petrop. 18(1): 1-146. [In Russian] (Лunский В. И. Материалы для флоры Средней Азии. I // Труды имп. Санкт-Петербургск. бот. сада, 1900. Т. 18, вып. 1. С. 1-146).

Lipsky V. I. 1904. Materials for the flora of Middle Asia. II. Acta Horti Petrop. 23(1): 1-247. [In Russian] (Лunский В. И. Материалы для флоры Средней Азии. II // Труды имп. Санкт-Петербургск. бот. сада, 1904. Т. 23, вып. 1. С. 1-247).

Lipsky V. I. 1910. Materials for the flora of Middle Asia. III. Acta Horti Petrop. 26(2): 115-616. [In Russian] (Липский В. И. Материалы для флоры Средней Азии. III // Труды имп. Санкт-Петербургск. бот. сада. 1910. Т. 26, вып. 2. С. 115-616).

Lowry P. P. II, Jones A. G. 1984. Systematics of Osmorhiza Raf. (Apiaceae: Apioideae) Annals of the Missouri Botanical Garden 71(4): 1128-1171.

Menitski Ju. L. 2008. Apiaceae Lindl. In: Conspectus florae caucasi. Ed. A. L. Takhtajan. Vol. 3a. St. Petersburg Moscow: KMK Scientific Press. Pp. 53-112. [In Russian] (Меницкий Ю. Л. Apiaceae Lindl. // Конспект флоры Кавказа. Ред. А. Л. Тахтаджян. Т. За. СПб.-М.: Изд-во КМК, 2008. С. 53-112).

Meusel H. 1971. Mediterranean elements in the flora and vegetation of the West Himalaya. In: Plant life of South West Asia. Eds. P. H. Davis, P. C. Harper, I. C. Hedge. Edinburgh: Royal Botanical Society. Pp. 53-72.

Mozaffarian V. 2007. Umbelliferae. In: Flora of Iran. Eds. M. Assadi, M. Khatamsaz, A. A Maassoumi. Tehran: Research Institute of Forests and Rangelands. 596 pp. [In Farsi].

Neves S. S., Snogerup S. 2001. Lectotype of Bupleurum lancifolium. In: S. Snogerup, B. Snogerup Bupleurum L. (Umbelliferae) in Europe - 1. The annuals, B. sect. Bupleurum and sect. Aristata. Willdenowia 31: 205-308.

Osten-Saken F., Ruprech F. J. 1869. Sertum Tianschanicum. Botanische Ergebnisse einer Reise im mittleren Tian-Schan. Mém. Acad. Imp. Sci. St.-Pétersbourg, ser. 7. 14(4): 1-74.

Pardó C. 1981. Estudio sistemático del género Seseli L. (Umbelliferae) in la peninsula Ibérica. Lazaroa 3: 163182.

Pavlov N. V. 1935. Umbelliferae [Ferula by E. P. Korovin]. In: Flora Centralnogo Kazakhstana [Flora Central Kazakhstan]. Vol. 2. Alma-Ata: Kazakhsk. Inst. Udobreniy i Agropochvovedeniya. Pp. 488-546. [In Russian] (Iarлов Н. В. Umbelliferae [Ferula. Автор Е. П. Коровин] // Флора Центрального Казахстана. Т. 2. Алма-Ата: Казахст. Инст. удобрений и агропочвоведения, 1935. С. 488-546).

Peev D. 1987. Torilis. In: Flora Iranica. Ed. K. H. Rechinger. Vol. 162. Graz: Akademische Druck-u.Verlagsanstalt. Pp. 121-130.

Pimenov M. G. 1974. De generis Seseli L. speciebus nonnullis in montibus Asiae Mediae cisferganensibus crescentibus. Novosti sistematiki vysshikh rasteniy [Novit. Syst. Pl. Vasc.] 11: 241-247. [In Russian, Latin] (Пименов 
M. Г. О видах рода Seseli, распространенных в горах Средней Азии, окружающих Ферганскую долину // Новости сист. высш. раст., 1974. Т. 11. С. 241-247).

Pimenov M. G. 1976. The genera Zosima Hoffm. and Pilopleura Schischk. in mountains of Middle Asia and Kazakhstan. Byull. Glavn. Bot. Sada. 101: 44-51. [In Russian] (Пименов М. Г. Роды Zosima Hoffm. и Pilopleura Schischk в горах Средней Азии // Бюл. ГБС, 1976. Вып. 101. С. 44-51).

Pimenov M. G. 1977. The new species and new combinations in the genus Seseli L. (Umbelliferae). Byull. Moskovsk. Obshch. Isp. Prir., Otd. Biol. 82, 3: 138-142. [In Russian] (Пименов М. Г. Новые виды и комбинации в роде Seseli L. (Umbelliferae). (Umbelliferae) // Бюл. МОИП. Отд. Биол., 1977. Т. 82, № 3. С. 138-142).

Pimenov M. G. 1978. De generis Seseli L. notulae systematicae. II. Adumbratio specierum florae URSS. Novosti sistematiki vysshikh rasteniy [Novit. Syst. Pl. Vasc.] 15: 188-200. [In Russian] (Пименов М. Г. К систематике рода Seseli L. II. Конспект видов флоры СССР // Новости сист. высш. раст., Т. 15. С. 188-200).

Pimenov M. G. 1979. A review of species of section Euryangium (Kauffm.) M. Pimen. of genus Ferula L. Byull. Moskovsk. Obshch. Isp. Prir., Otd. Biol. 84, 5: 106-111. [In Russian] (Пименов М. Г. Обзор видов секции Eигуапgium (Kauffm.) M. Рimen. рода Ferula L. // Бюл. МОИП. Отд. Биол., 1979. Т. 84, Вып. 5. С. 106-111).

Pimenov M. G. 1983. Umbelliferae. In: Conspectus florae Asiae Mediae. Vol. 7. Ed. A. I. Vvedensky. Tashkent: FAN. Pp. 167-322. [In Russian] (Пименов М. Г. Umbelliferae. Определитель растений Средней Азии. Критический конспект флоры. Т. 7. Ред. А. И. Введенский. Ташкент: ФАН, 1983. С. 167-322).

Pimenov M. G. 1988. Monographic revision of the genus Dorema D. Don (Umbelliferae). Byull. Moskovsk. Obshch. Isp. Prir., Otd. Biol. 93, 2: 76-90. [In Russian] (Пименов М. Г. Монографическая ревизия рода Dorema D. Don (Umbelliferae) // Бюл. МОИП. Отд. биол., 1988. Т. 93, вып. 2. С. 76-90).

Pimenov M. G. 2016. In: A. R. Kurbonov, M. G. Pimenov. The Umbelliferae of Northern Tajikistan: annotated checklist. Aegopodium - Galagania. Bot. Zhurn. (Moscow \& St. Petersburg) 101(10): 1220-1239. [In Russian] (Iuменов М. Г. в Курбонов А. Р., Пименов М. Г. Зонтичные Северного Таджикистана: Аннотированный конспект Aegopodium - Galagania // Бот. журн., 2016. Т. 101, № 10. С. 1220-1239).

Pimenov M. G. 2017a. Proposal to conserve the name Carum persicum (Bunium persicum, Elwendia persica) against Sium cyminosma (Umbelliferae). Taxon 66(3): 762.

Pimenov M. G. 2017b. Updated checklist of Chinese Umbelliferae: nomenclature, synonymy, typification, distribution. Turczaninowia 20, 2: 106-239. DOI: 10.14258/turczaninowia.20.2.9.

Pimenov M. G. 2018. Updated checklist of Chinese Umbelliferae: nomenclature, synonymy, typification, distribution. Supplementum. Turczaninowia 21, 1: 113-123. DOI: 10.14258/turczaninowia.21.1.10

Pimenov M. G., Kljuykov E. V. 1981. A contribution to the taxonomy of the genera Korshinskya, Physospermum, Astomatopsis and related genera of the Umbelliferae - Smyrniae s. str. Bot. Zhurn. (Moscow \& Leningrad) 66(4): 465-482. [In Russian] (Пименов М. Г., Клюйков Е. В. Материалы к систематике Korshinskya, Physospermum, Astomatopsis и близких родов Umbelliferae - Smyrniae s. str. // Бот. журн., 1981. Т. 66. № 4. C. 465-482).

Pimenov M. G., Kljuykov E. V. 1982. Critical analysis of the genera Hyalolaena and Hymenolyma and the related taxa of Umbelliferae - Apioideae]. Bot. Zhurn. (Moscow \& Leningrad) 67(7): 873-889. [In Russian] (Пименов М. Г., Клюйков Е. В. Критический анализ родов Hyalolaena и Нymenolyma и близких родов Umbelliferae - Apioideae // Бот. журн., 1982. Т. 67, № 7. С. 873-889).

Pimenov M. G., Kljuykov E. V. 2002. The Umbelliferae of Kirghyzia. Moscow: KMK Scientific Press. 286 pp. [In Russian] (Пименов М. Г., Клюйков Е. В. Зонтичные (Umbelliferae) Киргизии. М.: КМК, 2002. 286 с.).

Pimenov M. G., Kljuykov E. V. 2015. A new species of Bupleurum (Umbelliferae) from Badakhshan, with the notes and a key to alpine species of High Asia. Phytotaxa, Magnolia Press 226(3): 245-252. DOI: 10.11646/phytotaxa.226.3.4

Pimenov M. G., Kljuykov E. V., Degtjareva G. V. 2012. Note on two Umbelliferae taxa of South-Eastern Kazakhstan, invalidly published by M. S. Bajtenov. Bot. Zhurn. (Moscow \& St. Petersburg) 97(5): 650-658. [In Russian] (Пименов М. Г., Клюйков Е. В., Дегтярева Г. В. Заметка о двух таксонах зонтичных юго-восточного Казахстана, намеченных к описанию М. С. Байтеновым // Бот. журн., 2012. Т. 97, № 5. С. 650-658).

Pimenov M. G., Leonov M. V. 1993. The genera of the Umbelliferae. A nomenclator. Kew: Royal Botanic Gardens. 156 pp.

Pimenov M. G., Leonov M. V. 2002. The Asian Umbelliferae biodiversity in a mirror of computer database ASIUM. VI. In: Plant Life of Southwest Asia Symposium. Abstracts. Turkey: Van. P. 29.

Pimenov M. G., Leonov M. V. 2004. Asia, the continent with highest Umbelliferae biodiversity. South African Journal of Botany 70(3): 417-419.

Pimenov M. G., Ostroumova T. A. 2012. Umbelliferae of Russia. Moscow: KMK Scientific Press. 477 pp. [In Russian] (Пименов М. Г., Остроумова Т. А. Зонтичные (Umbelliferae) России. М.: КМК, 2012. 477 с.).

Pimenov M. G., Sdobnina L. I. 1973. On the taxonomic position of Pachypleurum mucronatum (Schrenk) Schischk.]. Byull. Moskovsk. Obshch. Isp. Prir., Otd. Biol. 78 (4): 132-140. [In Russian] (Пименов М. Г., Сдобнина Л. И. О таксономическом положении Pachypleurum mucronatum (Schrenk) Schischk. // Бюл. МОИП. Отд. Биол., 1973. T. 78, № 4.C. 132-140). 
Pimenov M. G., Sutorý K. 2014. Name typification of Umbelliferae taxa described by Joseph Franz Freyn. Plant Biosystems 148(5-6): 1102-1111.

Pimenov M. G., Tamamschjan S. G. 1987. Eryngium. In: Flora Iranica. Ed. K. H. Rechinger. Vol. 162. Graz: Akademische Druck-u.Verlagsanstalt. Pp. 45-60.

Pimenov M. G., Tikhomirov V. N. 1983. The taxonomic problems in the genera Prangos Lindl., Cachrys L., Cryptodiscus Schrenk and Hippomarathrum Hoffmgg. et Link. Feddes Repertorium 94 (3-4): 145-164.

Plunkett G. M., Pimenov M. G., Reduron J.-P., Kljuykov E. V., Wyk B.-E. van, Ostroumova T. A., Henwood M. J., Tilney P. M., Spalik K., Watson M. F., Lee B.-Y., Pu F.-D., Webb C. J., Hart J. M., Mitchell A. D., Muckensturm B. 2019. Apiaceae Lindl., Intr. Nat. Syst. Bot., ed. 2: 21 (1836), nom. cons. et nom. alt. Umbelliferae Juss., Gen. Pl.: 218 (1789), nom. cons. et nom. alt. In: Families and genera of vascular plants. Vol. 15. Ed. K. Kubitzki. Pp. 9-206.

Polozhij A. V., Balaschova V. F. 1989. Tipy taksonov v Gerbarii imeni P. N. Krylova [Types of taxa in Krylov Herbarium]. Tomsk. 47 pp. [In Russian] (Положий А. В., Балашова В. Ф. Типы таксонов в Гербарии им. П. Н. Крылова. Томск, 1989. 47 с.).

Rakhmankulov U., Melibaev S. 1982. Generis Ferula L. (Apiaceae) species nova e montibus Nuratau. Novosti sistematiki vysshikh rasteniy [Novit. Syst. Pl. Vasc.] 19: 117-119. [In Russian] (Рахманкулов У., Мелибаев С. Новый вид рода Ferula L. (Apiaceae) с гор Нуратау // Новости сист. высш. раст., 1982. Т. 19. С. 117-119).

Rechinger K. H. 1987. Falcaria, Seseli, Lomatopodium, Libanotis. In: Flora Iranica. Ed. K. H. Rechinger. Vol. 162. Graz: Akademische Druck-u.-Verlagsanstalt. Pp. 306-308, 347-350, 351-355.

Rechinger K. H., Snogerup S. 1987. Bupleurum (species perennes \& species annuae). In: Flora Iranica. Ed. K. H. Rechinger. Vol. 162. Graz: Akademische Druck-u.-Verlagsanstalt. Pp. 269-297.

Reduron J.-P. 2007-2008. Ombellifères de France. Vol. 1-5. Bulletin de la Société Botanique du Centre-Ouest, Nouvelle Série, num. spéc. 26: 1-564; 27: 565-1142; 28: 1143-1726; 29: 1727-2348; 30: 2349-3004.

Reduron J.-P., Charpin A., Pimenov M. 1997. Contribution à la nomenclature générique des Apiaceae (Ombellifères) Journal de Botanique de la Société Botanique de France 1: 91-104.

Reduron J.-P., Jarvis C. E. 1992. Angelica, Apium, Chaerophyllum. In: Seventy-two proposals for the conservation of types of selected Linnean generic names, the report of Subcommitee 3C on the lectotypification of Linnean generic names. Ed. D. H. Nicolson. Taxon 41(3): 552-583

Regel E., Herder F. 1866. Enumeratio plantarum in regionibus cis- et transiliensibus a cl. Semenowio anno 1857 collectarum. Bulletin de la Société Impériale des Naturalistes de Moscou 39(3): 527-571.

Regel E., Schmalhausen I. F. 1878a. Descriptiones plantarum novarum vel minus cognitarum. Fasc. 6. Trudy Imp. S.-Peterburgsk. Bot. Sada 5(2): 575-646.

Regel E., Schmalhausen I. F. 1878b. Ferula foetidissima Rgl. et Schmalh. Umbelliferae. Gartenflora 27: 195-199.

Regel E., Schmalhausen I. F. 1882. Umbelliferae. In: E. Regel. Descriptiones plantarum novarum rariorumque a cl. Olga Fedtschenko in Turkestania nec non in Kokania lectarum. Izv. Imp. Obshch. Lyubit. Estestv. Moskovsk. Univ. 34 (2): 27-41.

Saenz Lain C. 1981. Research on Daucus L. (Umbelliferae) Anales del Jardin Botánico de Madrid 37(2): 481-533.

Schischkin B. K. 1950. Umbelliferae. In: Flora SSSR [Flora of the USSR]. Ed. B. K. Schischikin. Vol. 16. Moscow-Leningrad: Publishers of Academy of Sciences of USSR. Pp. 36-604. [In Russian] (Шишкин Б. К. Umbelliferae // Флора СССР. Т. 16. Под ред. Б. К. Шишкина. М.-Л.: Акад. Наук СССР, 1951. С. 36-604).

Schischkin B. K. 1951. Umbelliferae. In: Flora SSSR [Flora of the USSR]. Ed. B. K. Schischikin. Vol. 17. MoscowLeningrad: Publishers of Academy of Sciences of USSR. Pp. 1-359. [In Russian] (Шиикин Б. К. Umbelliferae // Флора СССР. Т. 17. Под ред. Б. К. Шишкина. М.-Л.: Акад. Наук СССР. 1951. С. 1-359).

Schrenk A. 1843. Plantae novae, nondum descriptae, quas in itinere ad fluvium Tschu versus legit Al. Schrenk. Bulletin de la Classe Physico-Mathématique de l'Academie Impériale des Sciences de Saint-Petersbourg 2 (8): 113-116.

Solovyev S. M. 1851. History of Russia since ancient times in 29 volumes. T. 1. Ch. 1. 331 pp. [In Russian] (Cоловьев С. М. История России с древнейших времен. В 29 тт. Т. 1. Гл. 1. 331 с. (according modern electronic edition 2016. Direct-media https://biblioclub.ru/index./php)

Spalik K., Jarvis C. E. 1989. Typification of Linnean names now in Anthriscus (Apiaceae). Taxon 38(2): $288-293$.

Strid A. 2000. New taxa descrided in Grisebach's "Spicilegium Florae Rumeliae et Bithinicae" (1843-1846). Preslia 72: 240-321.

Takhtajan A. L. 1978. Floristicheskiye oblasti Zemli [Floristic regions of the World]. Leningrad: Nauka. 247 pp. [In Russian] (Тахтаджян A. Л. Флористические области Земли. Л.: Наука, 1978. 247 с.).

Tardieu-Blot M.-L. 1967. Umbelliferae. In: Flore du Cambodge, du Laos et du Vietnam.Vol. 5. Ed. M.-L. Tardieu-Blot. Paris: Muséum National d'Histoire Naturelle. Pp. 1-77.

The Times Comprehensive Atlas of the World. 2011. Edition $13^{\text {th }}$. Harper Collins Publisher.

Tojibaev K. Sh., Beshko N. Ju., Turginov O. T., Lyskov D. F., Ukrainskaja U. A., Kljuykov E. V. 2017. An annotated checklist of the endemic Apiaceae of Uzbekistan. Phytotaxa 455(2): 70-94. DOI: 10.11646/phytotaxa.455.2.2

Townsend C. C. 1966. Notes on the Umbelliferae of Iraq: III. Kew Bulletin 20(1): 77-85. 
Townsend C. C. 1989. Umbelliferae. In: Flora of Tropical East Africa. Ed. R. M. Polhill. Rotterdam: A. A. Balkema. Pp. 1-127.

Wijnheimer E. H. M., Brandenhyburg W. A., Jarvis C. E. 1988. Lectotypification of Daucus carota L. (Umbelliferae). Taxon 37: 175-184.

Vinogradova V. M. 1997a. Type specimens of Apiaceae taxa from Middle Asia, kept in Herbarium of Komarov Botanical institute (LE). 1. Angelica - Hyalolaena. Bot. Zhurn. (Moscow \& St. Petersburg) 82, 3: 95-102. [In Russian] (Виноградова В. М. Типовые образцы таксонов семейства Аріасеае Средней Азии, хранящиеся в Гербарии Ботанического института им. В. Л. Комарова (LE). 1. Angelica-Hyalolaena // Бот. журн., 1997. Т. 82 , № 3. C. 95-102).

Vinogradova V. M. 1997b. Type specimens of Apiaceae taxa from Middle Asia, kept in Herbarium of Komarov Botanical institute (LE). 2. Hymenolaena - Seselopsis. Bot. Zhurn. (Moscow \& St. Petersburg) 82, 4: 53-61. [In Russian] (Виноградова В. М. Типовые образцы таксонов семейства Аріасеае Средней Азии, хранящиеся в Гербарии Ботанического института им. В. Л. Комарова (LE). 2. Hymenolaena - Seselopsis // Бот. журн., 1997. T. 82, № 4. С. 53-61).

Vinogradova $V$. M. 1999. Type specimens of Apiaceae taxa from Middle Asia, kept in Herbarium of Komarov Botanical institute (LE). 3. Aegopodium - Eryngium. Bot. Zhurn. (Moscow \& St. Petersburg) 84, 3: 80-87. [In Russian] (Виноградова В. М. Типовые образцы таксонов семейства Аріасеае Средней Азии, хранящиеся в Гербарии Ботанического института им. В. Л. Комарова (LE) 3. Aegopodium - Eryngium // Бот. журн., 1999. Т. 84, № 3. C. 80-87).

Vinogradova $\boldsymbol{V}$. M. 2000. Type specimens of Apiaceae taxa from Middle Asia, kept in Herbarium of Komarov Botanical institute (LE). 4. Ferula. Bot. Zhurn. (Moscow \& St. Petersburg) 85, 1: 89-96. [In Russian] (Виноградова B. M. Типовые образцы таксонов семейства Аріасеае Средней Азии, хранящиеся в Гербарии Ботанического института им. В. Л. Комарова (LE). 4. Ferula // Бот. журн., 2000. Т. 85, № 1. С. 89-96).

Vinogradova $\boldsymbol{V}$. M. 2001. Type specimens of Apiaceae taxa from Middle Asia, kept in Herbarium of Komarov Botanical institute (LE) 5. Elaeosticta-Prangos. Bot. Zhurn. (Moscow \& St. Petersburg) 86, 2: 50-57. [In Russian] (Виноградова В. М. Типовые образцы таксонов семейства Аріасеае Средней Азии, хранящиеся в Гербарии Ботанического института им. В. Л. Комарова (LE) 5. Elaeosticta - Prangos) // Бот. журн., 2001. Т. 86, № 2. C. 50-57).

Vinogradova $\boldsymbol{V}$. M. 2002. Type specimens of Apiaceae taxa from Middle Asia, kept in Herbarium of Komarov Botanical institute (LE) 6. Reutera - Zozima. Bot. Zhurn. (Moscow \& St. Petersburg) 87, 6: 132-140. [In Russian] (Виноградова В. М. Типовые образцы таксонов семейства Аріасеае Средней Азии, хранящиеся в Гербарии Ботанического института им. В. Л. Комарова (LE). 6. Reutera-Zozima) // Бот. журн., 2002. Т. 87, № 6. C. 132-140).

Vinogradova V. M. 2003. Type specimens of Apiaceae taxa from East Europe, kept in the Herbarium of Komarov Botanical institute of RAS (LE) (Anthriscus - Trinia). Bot. Zhurn. (Moscow \& St. Petersburg) 88, 8: 105-111. [In Russian] (Виноградова B. М. Типовые образцы таксонов семейства Аріасеае Восточной Европы, хранящиеся в Гербарии Ботанического института им. В. Л. Комарова (LE) (Anthriscus - Trinia) // Бот. журн., 2003. Т. 88, № 8. C. 105-111).

Vinogradova V. M. 2005. Type specimens of Apiaceae taxa from Siberia and North-Eastern Kazakhstan, kept in Herbarium of Komarov Botanical institute (LE) (Aegopodium - Stenocoelium). Bot. Zhurn. (Moscow \& St. Petersburg) 90(6): 957-967. [In Russian] (Виноградова В. М. Типовые образцы таксонов семейства Аріасеае Сибири и северо-восточного Казахстана, хранящиеся в Гербарии Ботанического института им. В. Л. Комарова (LE) (Aegopodium - Stenocoelium) // Бот. журн., 2005. Т. 90, № 6. С. 957-967).

Vinogradova V. M. 2007. Notula de material typica speciei Ledebouriella multiflora (Ledeb.) H. Wolff (Apiaceae). Novosti sistematiki vysshikh rasteniy [Novit. Syst. Pl. Vasc.] 39: 260-272. [In Russian] (Виноградова В. М. Заметки о типовом материале Ledebouriella multiflora (Ledeb.) H. Wolff (Арiaceae) // Новости сист. высш. раст., 2007. T. 39. C. 260-272).

Wörz A. 1999. A taxonomic index of the species of Eryngium (Apiaceae: Saniculoideae). Stuttgarter Beiträge zur Naturkunde. Serie A (Biologie). № 596. $48 \mathrm{~S}$.

Wörz A. 2011. Revision of Eryngium L. (Apiaceae - Saniculoideae): General part and Palaeoarctic species. In: Bibliotheca Botanica. Vol. 159. Stuttgart: Schweizerbart Science Publishers. 498 pp.

Yurtzeva O. V. 1995. On the system of Pimpinella L. subgenus Tragium (Spreng.) Reichenb. (Umbelliferae Juss.). Byull. Moskovsk. Obshch. Isp. Prir., Otd. Biol. 100, 3: 61-72. [In Russian] (Юрцева О. В. К системе подрода Tragium (Spreng.) Reichenb. рода Pimpinella L. (Umbelliferae Juss.) // Бюл. МОИП. Отд. биол., 1985. Т. 100, вып. 3. C. 61-72). 